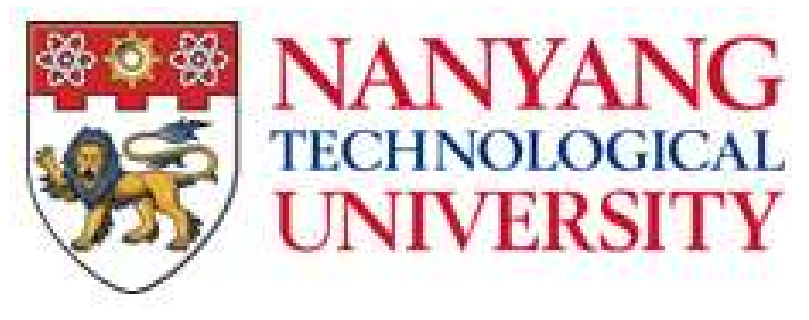

\title{
The Regulation of Cancer Cachexia and Colorectal Carcinogenesis by Myostatin
}

\author{
Isuru Wijerupage Wijesoma \\ G1202585G
}

School of Biological Sciences

2017

A report submitted to the School of Biological Sciences in partial fulfilment for the Doctor of Philosophy Degree 


\section{Acknowledgements}

My thesis was born out of a very difficult time in my professional career. As such, I would not be at this stage without the support of several key individuals in my life. First, I would like to thank Professor Koh Cheng Gee for her guidance and support. Second, I am grateful for Professor Alex Law for his critique and comments on my thesis. Furthermore, I would like to thank Professor Surajit Bhattacharya for choosing to be my current supervisor. And of course, my father, Amma, Mali and Nangi for their unwavering support.

I would also like to express my gratitude towards the School of Biological Sciences (SBS), Nanyang Technological University (NTU), the staff at the Animal House Facility, Singapore Institute for Clinical Sciences (SICS), and the Singapore International Graduate Award (SINGA) for supporting my scientific research and education. I would also like to thank Professor Se-Jin Lee from Johns Hopkins University School of Medicine for generously providing us the $\mathrm{Mstn}^{+/-}$mice. Furthermore, I would like to thank Esther Latres from Regeneron for providing the MuRF1 antibody.

I would like to thank Professor Ravi Kambadur, Professor Mridula Sharma, and PI Dr. Craig McFarlane for their mentorship and assistance during the course of my doctorate. I would like to also thank Professor Nick Barker for sharing his expertise in intestinal biology. I would like to thank Bhagi Somalanka for being an extremely hardworking collaborator. Her assistance and support in this project have been invaluable. Additionally, I would also like to thank Dr. Piyush Khandelia. His advice, mentorship and friendship have made an indelible mark on my work and life. I would also like to express my appreciation for several members of my laboratory, Gavian, Nadhya, Holly, and many others.

And finally, my dearest and beloved wife, Chitra. Words cannot express how eternally thankful for your presence in my life. 


\section{Table of Contents}

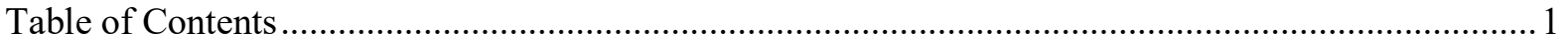

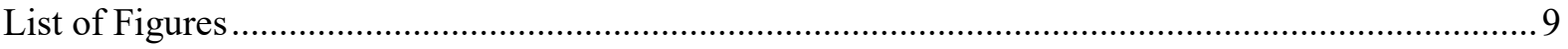

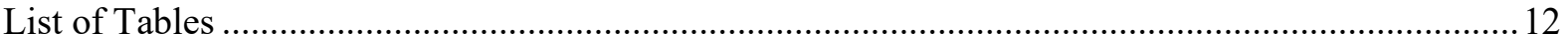

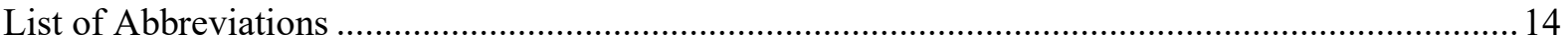

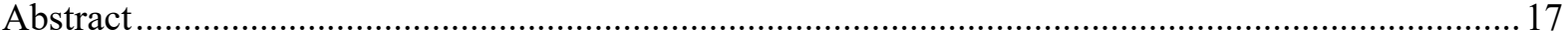

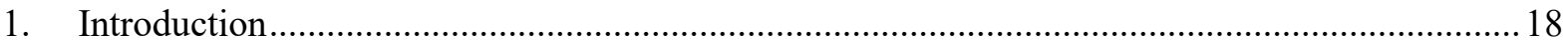

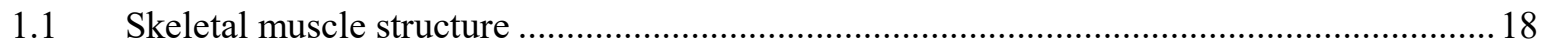

Figure 1.1.1: The structure of the skeletal muscle..................................................................... 19

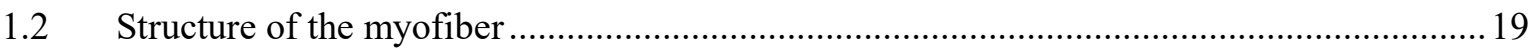

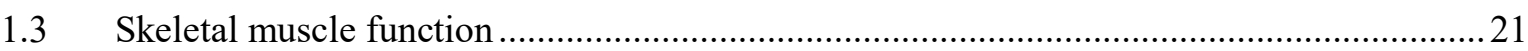

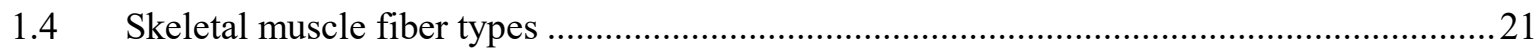

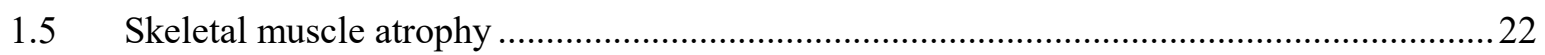

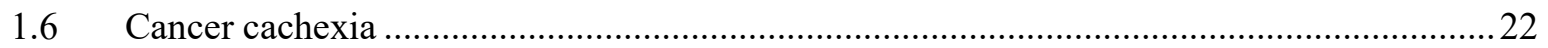

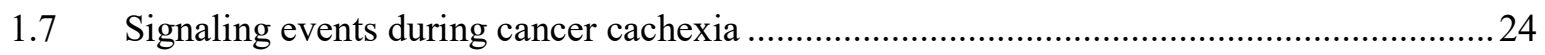

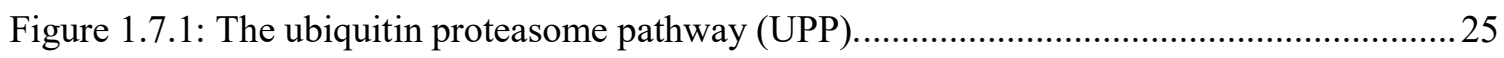

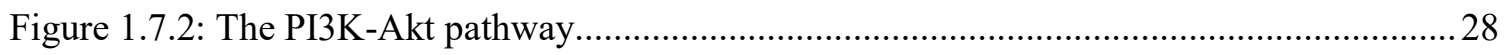

$1.8 \quad$ Extracellular signaling molecules involved in cancer cachexia .............................................30

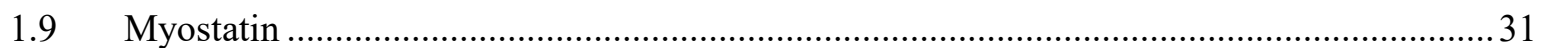

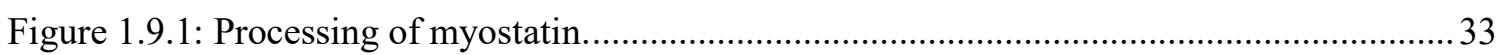

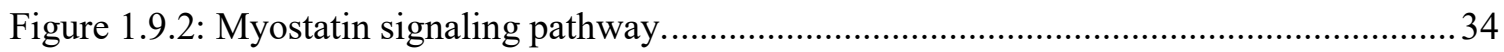

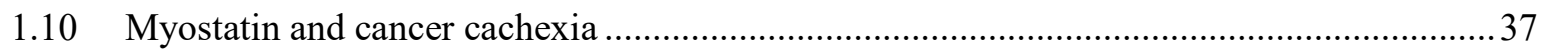

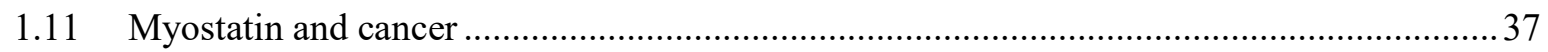

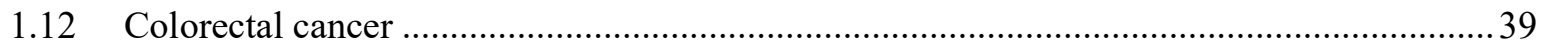

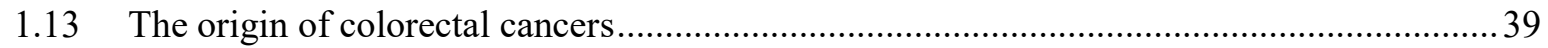

1.12 Models to study colorectal cancer and cancer cachexia....................................................... 40

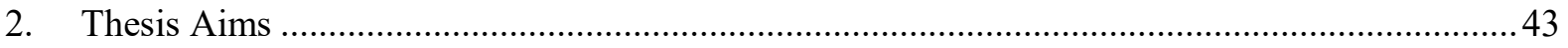

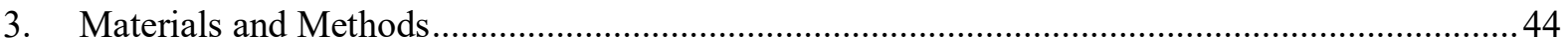

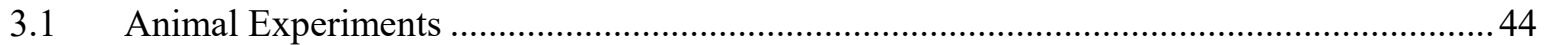

3.2 Genomic DNA isolation from mouse ear tissue ............................................................... 44

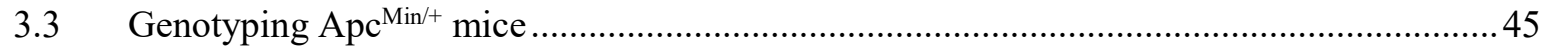

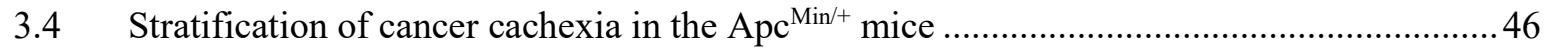

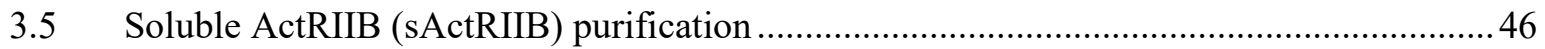

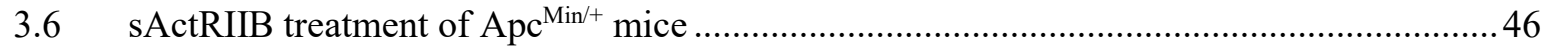


3.7 Grip strength measurement

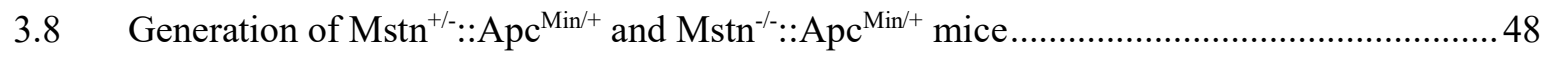

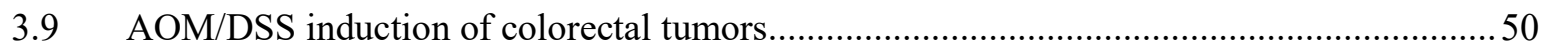

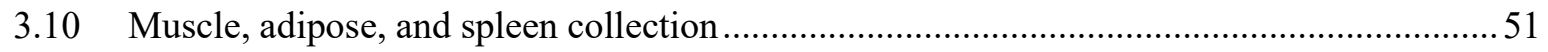

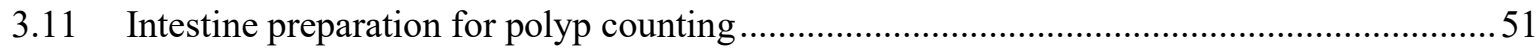

3.12 Organoid culture of $\mathrm{Mstn}^{+/+}:: \mathrm{Apc}^{\mathrm{Min} /+}, \mathrm{Mstn}^{+/}:: \mathrm{Apc}^{\mathrm{Min} /+}$ and $\mathrm{Mstn}^{-/}:: \mathrm{Apc}^{\mathrm{Min} /+}$ small intestine

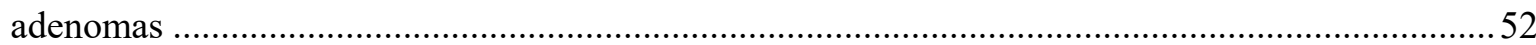

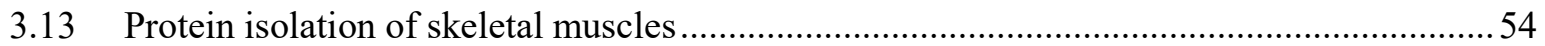

3.14 Protein isolation from $\mathrm{Mstn}^{+/+}:: \mathrm{Apc}^{\mathrm{Min} /+}, \mathrm{Mstn}^{+/-}:: \mathrm{Apc}^{\mathrm{Min} /+}$ and $\mathrm{Mstn}^{-/ /}:: \mathrm{Apc}^{\mathrm{Min} /+}$ organoids. 54

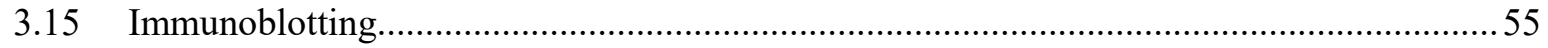

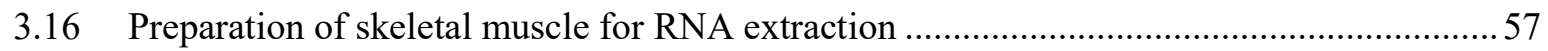

3.17 Preparation of intestinal tissue and polyps for RNA extraction........................................57

3.18 RNA isolation from $\mathrm{Mstn}^{+/+}:: \mathrm{Apc}^{\mathrm{Min} /+}, \mathrm{Mstn}^{+/ .}:: \mathrm{Apc}^{\mathrm{Min} /+}$ and $\mathrm{Mstn}^{-/ .}:: \mathrm{Apc}^{\mathrm{Min} /+}$ organoids .... 58

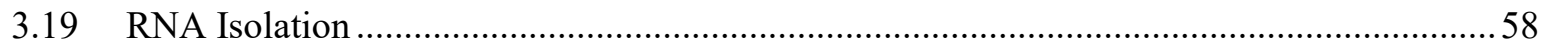

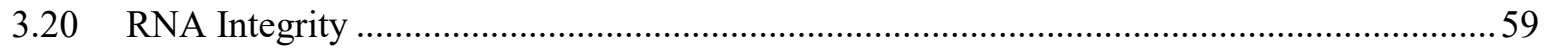

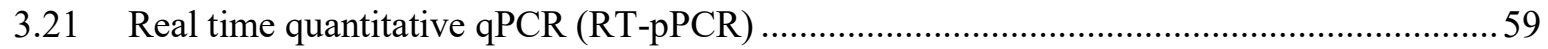

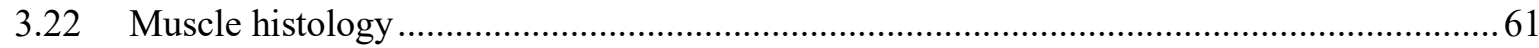

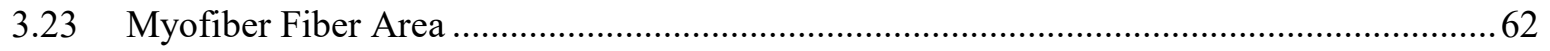

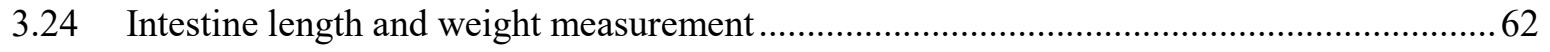

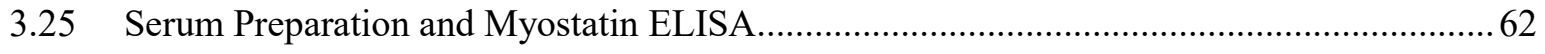

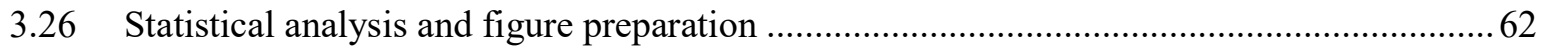

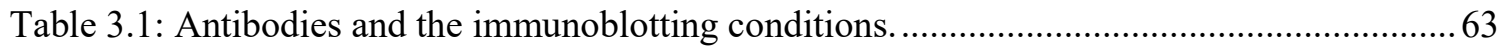

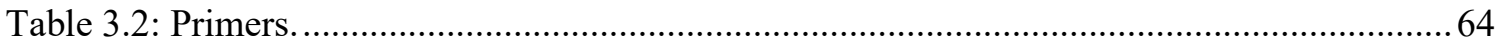

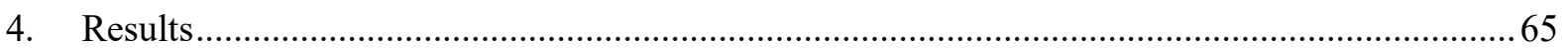

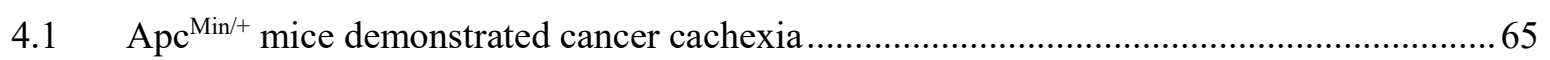

Figure 4.1.1: Aging male $\mathrm{Apc}^{\mathrm{Min} /+}$ mice displayed body weight loss..........................................66

Table 4.1.1: Body weights of $\mathrm{Apc}^{\mathrm{Min} /+}$ mice stratified by their percentage body weight loss .......68

4.2 Cachectic $\mathrm{Apc}^{\mathrm{Min} /+}$ mice displayed skeletal muscle and adipose tissue atrophy ...................69

Figure 4.2.1: Cachectic $\mathrm{Apc}^{\mathrm{Min} /+}$ mice demonstrated skeletal muscle atrophy. ............................. 70

Figure 4.2.2: Cachectic $\mathrm{Apc}^{\mathrm{Min} /+}$ mice exhibited adipose tissue atrophy. ................................... 71

4.3 Skeletal muscle atrophy in cachectic $\mathrm{Apc}^{\mathrm{Min} /+}$ mice was associated with the increased

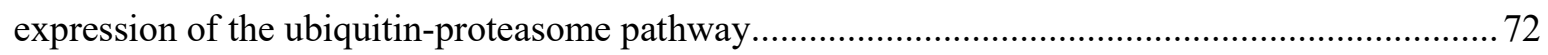

Figure 4.3.1: Increased levels of Atrogin-1 and Murf1 mRNA was associated with increasing

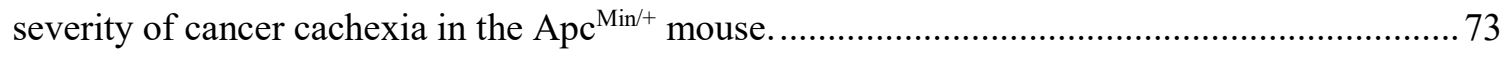

Figure 4.3.2: Elevated protein expression of key mediators of the ubiquitin-proteasome pathway was associated with increasing severity of cancer cachexia in the $\mathrm{Apc}^{\mathrm{Min} /+}$ mouse. 
4.4 Myostatin gene expression was elevated in the skeletal muscles of cachectic $\mathrm{Apc}^{\mathrm{Min} /+}$ mice ..

Figure 4.4.1: Myostatin gene expression levels were elevated in the GAS muscles of moderately

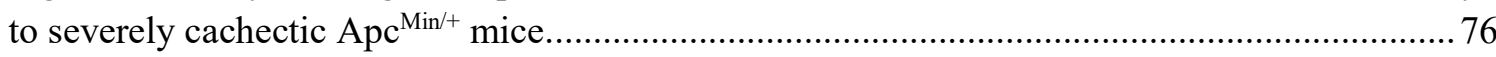

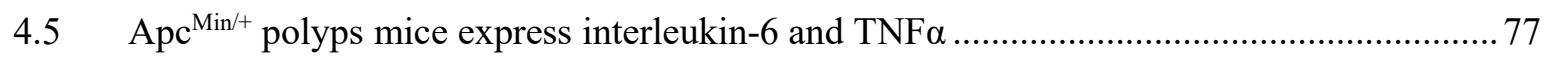

Figure 4.5.1: Small intestine polyps from $\mathrm{Apc}^{\mathrm{Min} /+}$ mice express IL-6 and TNF $\alpha \ldots \ldots \ldots \ldots \ldots \ldots \ldots \ldots . . . . . . . . . . . .18$

Figure 4.5.2: Small intestine polyp number did not correlate with male $\mathrm{Apc}^{\mathrm{Min} /+}$ body weight or

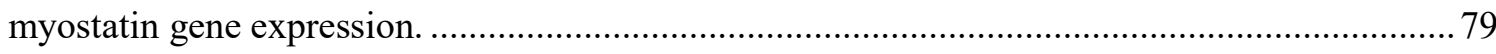

4.6 Soluble ActRIIB administration partially halted the loss of skeletal muscle mass and

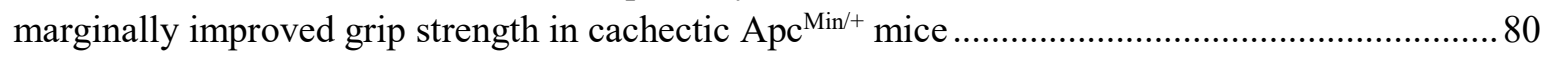

Figure 4.6.1: The loss of body mass due to cancer cachexia in male $\mathrm{Apc}^{\mathrm{Min}+}$ mice was not

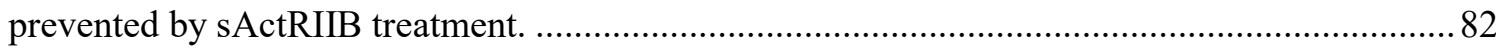

Figure 4.6.2: Soluble ActRIIB treatment of male $\mathrm{Apc}^{\mathrm{Min} /+}$ mice at 16 weeks partially prevented skeletal muscle atrophy and improved forelimb grip strength.

Figure 4.6.3: Soluble ActRIIB treatment of male $\mathrm{Apc}^{\mathrm{Min} /+}$ mice at 16 weeks failed to improve the TA myofiber cross-sectional area.

Figure 4.6.4: Soluble ActRIIB treatment of pre-cachectic male $\mathrm{Apc}^{\mathrm{Min} /+}$ mice failed to prevent the

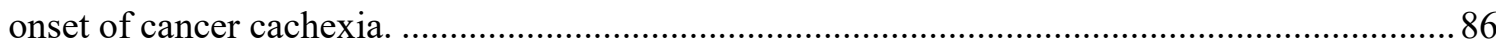

Figure 4.6.5: Soluble ActRIIB treatment of pre-cachectic male $\mathrm{Apc}^{\mathrm{Min} /+}$ mice partially improved forelimb grip strength.

Figure 4.6.6: The small intestine tumor biology of $\mathrm{Apc}^{\mathrm{Min} /+}$ mice administered with sActRIIB from 16 weeks of age.

4.7 The genetic ablation of myostatin prevented the onset of cancer cachexia in $\mathrm{Apc}^{\mathrm{Min} /+}$ mice 90

Figure 4.7.1: Genetic ablation of myostatin prevented the onset of cancer cachexia in 18 week old $\mathrm{Apc}^{\mathrm{Min} /+}$ mice.

Figure 4.7.2: TA Myofiber cross-sectional area of 18 week old $\mathrm{Mstn}^{+/-::} \mathrm{Apc}^{\mathrm{Min} /+}$ and $\mathrm{Mstn}^{-/}$ $:: \mathrm{Apc}^{\mathrm{Min} /+}$ mice.

Figure 4.7.3: The absence of myostatin suppressed the over-expression of the ubiquitin proteasome pathway in skeletal muscles of 18 week old $\mathrm{Mstn}^{+/-}:: \mathrm{Apc}^{\mathrm{Min} /+}$ and $\mathrm{Mstn}^{-/}:: \mathrm{Apc}^{\mathrm{Min} /+}$ mice.

Figure 4.7.4: Genetic ablation of myostatin preserved adipose tissue and spleen mass in 18 week old $\mathrm{Apc}^{\mathrm{Min} /+}$ mice

4.8 The deletion of myostatin did not alter the intestinal tumor biology of the $\mathrm{Apc}^{\mathrm{Min} /+}$ mouse 96

Figure 4.8.1: Genetic ablation of myostatin failed to alter the tumor biology of the $\mathrm{Apc}^{\mathrm{Min} /+}$ mouse.

4.9 The growth characteristics of $\mathrm{Mstn}^{-/}:: \mathrm{Apc}^{\mathrm{Min} /+}$ cancer organoids .....

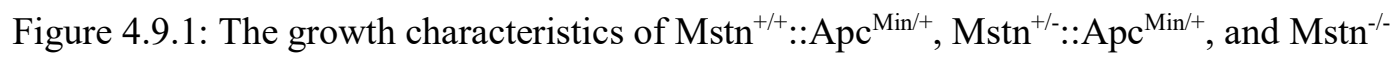

$:: \mathrm{Apc}^{\mathrm{Min} /+}$ small intestine cancer organoids.

4.10 $\mathrm{Mstn}^{-/}$mice were resistant to colitis-induced colorectal carcinogenesis. 
Figure 4.10.1: Body weights for male $\mathrm{Mstn}^{+/+}$and $\mathrm{Mstn}^{-/-}$mice subjected to the AOM/DSS model

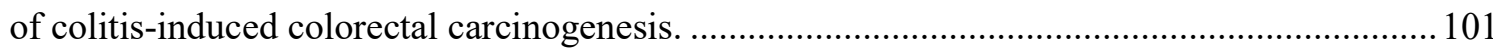

Figure 4.10.2: Absence of myostatin suppressed colitis-induced colorectal tumorigenesis....... 102

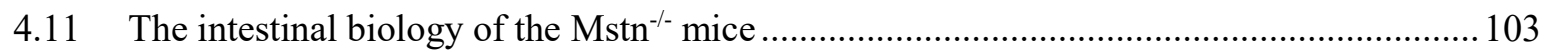

Figure 4.11.1: Intestinal biology of $\mathrm{Mstn}^{+/+}, \mathrm{Mstn}^{+/}$, and $\mathrm{Mstn}^{-/}$mice...................................... 104

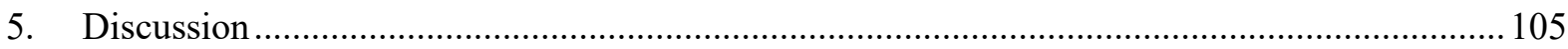

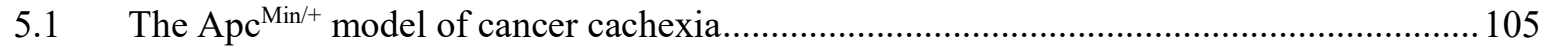

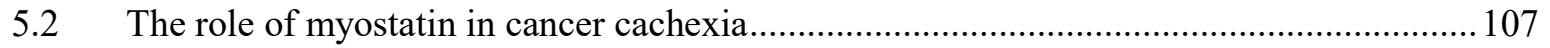

5.3 The inhibition of myostatin prevented cancer cachexia in the $\mathrm{Apc}^{\mathrm{Min} /+}$ mouse ................... 109

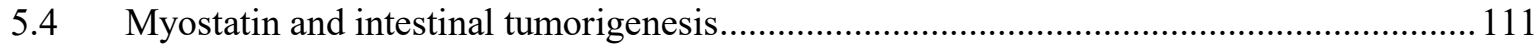

5.5 Murine genetic background and intestinal tumorigenesis................................................ 113

5.6 Future experiments to investigate the link between myostatin and cancer .........................115

Figure 5.6.1: Investigating the molecular mechanism of myostatin and cancer......................... 116

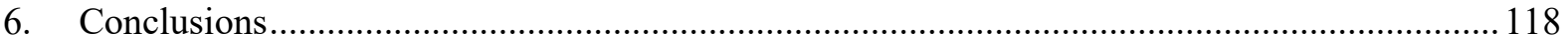

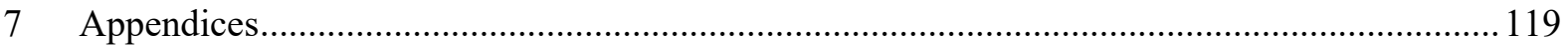

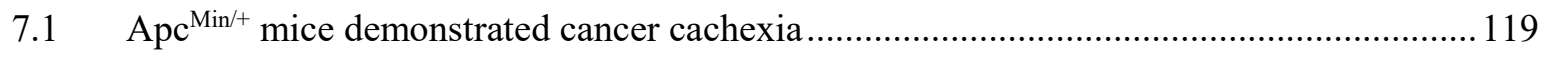

Table 7.1.1 (Section 4.1, Figure 4.1.1): Body weights for male $\mathrm{Apc}^{\mathrm{Min} /+}$ mice (n=9-50) and wild

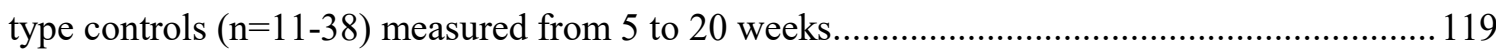

Table 7.1.2 (Section 4.1, Table 4.1.1): Male $\mathrm{Apc}^{\mathrm{Min} /+}$ mice used for the cancer cachexia stratification trial.

Table 7.1.3 (Section 4.1, Table 4.1.1): Tibia lengths $(\mathrm{mm})$ for wild type $(\mathrm{n}=6)$, non-cachectic

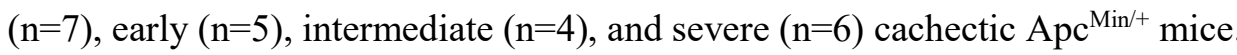

7.2 Cachectic $\mathrm{Apc}^{\mathrm{Min} /+}$ mice displayed skeletal muscle and adipose tissue atrophy

Table 7.2.1 (Section 4.2, Figure 4.2.1): Skeletal muscle weights of $\mathrm{Apc}^{\mathrm{Min} /+}$ mice stratified by percentage body weight loss

7.3 Skeletal muscle atrophy in cachectic $\mathrm{Apc}^{\mathrm{Min} /+}$ mice was associated with the increased

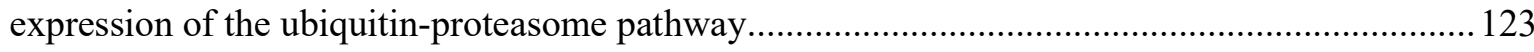

Figure 7.3.1 (Section 4.3, Figure 4.3.2A and 4.3.2B): Immunoblot for Atrogin-1.................... 123

Figure 7.3.2 (Section 4.3, Figures 4.3.2A and 4.3.2B): Immunoblot for p-FoxO3a................... 124

Figure 7.3.3 (Section 4.3): Immunoblot for GAPDH corresponding to Atrogin-1 and p-FoxO3a immunoblots represented in Figure 7.3.1 and Figure 7.3.2 (Section 4.3, Figure 4.3.2A and 4.3.2B).

Figure 7.3.4 (Section 4.3, Figures 4.3.2A and 4.3.2B): Immunoblot for FoxO3a immunoblots.126 Figure 7.3.5: Immunoblot for GAPDH corresponding to FoxO3a immunoblots represented in

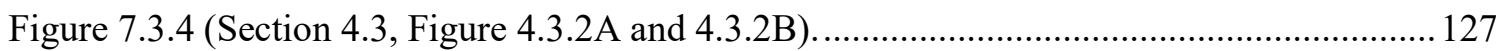

Figure 7.3.6 (Section 4.3, Figures 4.3.2A and 4.3.2B): Immunoblot for MuRF1...................... 128

Figure 7.3.7: Immunoblot for GAPDH corresponding to MuRF1 immunoblots represented in Figure 7.3.6 (Section 4.3, Figures 4.3.2A and 4.3.2B). 
7.4 Myostatin gene expression was elevated in the skeletal muscles of cachectic $\mathrm{Apc}^{\mathrm{Min} /+}$ mice

Figure 7.4.1 (Section 4.4, Figure 4.4.1): Myostatin gene expression levels were elevated in the GAS muscles of moderately to severely cachectic $\mathrm{Apc}^{\mathrm{Min} /+}$ mice.

Figure 7.4.2 (Section 4.4, Figure 4.4.1): Myostatin gene expression levels in the GAS muscles of

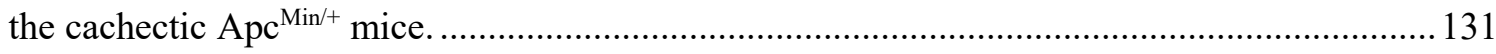

7.5 $\mathrm{Apc}^{\mathrm{Min} /+}$ polyps mice express interleukin- 6 and TNF $\alpha$

Figure 7.5.1 (Section 4.5, Figure 4.5.1): Small intestine polyps from $\mathrm{Apc}^{\mathrm{Min} /+}$ male mice express interleukin- 6 and TNF $\alpha$.

Figure 7.5.2 (Section 4.5, Figure 4.5.1): Small intestine polyps from $\mathrm{Apc}^{\mathrm{Min} /+}$ male mice do not express TNF $\alpha$ variant 2 .......

7.6 Soluble ActRIIB administration partially halted the loss of skeletal muscle mass and marginally improved grip strength in cachectic $\mathrm{Apc}^{\mathrm{Min} /+}$ mice

Table 7.6.1 (Section 4.6, Figures 4.6.1-4.6.3): Dosage of sActRIIB administered to $\mathrm{Apc}^{\mathrm{Min} /+}$ mice at 16 weeks.

Table 7.6.2 (Section 4.6, Figure 4.6.2A): Grip strength, measured in Newtons (N), for agematched wild type $(n=7)$, dialysis buffer $(n=11)$, and sActRIIB treated $\mathrm{Apc}^{\mathrm{Min} /+}(\mathrm{n}=9)$ mice.... 135

Figure 7.6.1 (Section 4.6, Figure 4.6.3): Soluble ActRIIB treatment of male $\mathrm{Apc}^{\mathrm{Min} /+}$ mice at 16 weeks failed to improve the TA myofiber cross-sectional area. 136

Table 7.6.3 (Section 4.6, Figure 4.6.2B): Skeletal muscle weights for dialysis buffer $(n=11)$ and sActRIIB treated $\mathrm{Apc}^{\mathrm{Min} /+}(\mathrm{n}=9)$ mice.

Table 7.6.4 (Section 4.6, Figure 4.6.2B): Skeletal muscle weights for dialysis buffer $(n=8)$ and sActRIIB treated $\mathrm{Apc}^{\mathrm{Min} /+}(\mathrm{n}=9)$ mice.

Table 7.6.5 (Section 4.6, Figures 4.6.1-4.6.3): Tibia length $(\mathrm{mm})$ for dialysis buffer $(\mathrm{n}=11)$ and sActRIIB treated $\mathrm{Apc}^{\mathrm{Min} /+}(\mathrm{n}=9)$ mice...

Table 7.6.6 (Section 4.6, Figures 4.6.4 and 4.6.5): Dosage of sActRIIB administered to Apc ${ }^{\mathrm{Min} /+}$ mice at 14 weeks.

Table 7.6.7 (Section 4.6, Figure 4.6.5B): Skeletal muscle weights for dialysis buffer $(n=6)$ and sActRIIB treated $\mathrm{Apc}^{\mathrm{Min} /+}(\mathrm{n}=5)$ mice.

Table 7.6.8 (Section 4.6, Figures 4.6.4 and 4.6.5): Tibia length $(\mathrm{mm})$ for dialysis buffer $(\mathrm{n}=6)$ and $\mathrm{sActRIIB}$ treated $\mathrm{Apc}^{\mathrm{Min} /+}(\mathrm{n}=5)$ mice.

7.7 The genetic ablation of myostatin prevented the onset of cancer cachexia in $\mathrm{Apc}^{\mathrm{Min} /+}$ mice

Table 7.7.1 (Section 4.7, Figure 4.7.1C): Skeletal muscle weights for $\mathrm{Mstn}^{+/+}:: \mathrm{Apc}^{\mathrm{Min} /+}(\mathrm{n}=6)$, $\mathrm{Mstn}^{+/}: \because \mathrm{Apc}^{\mathrm{Min} /+}(\mathrm{n}=4)$, and $\mathrm{Mstn}^{-/}: \because \mathrm{Apc}^{\mathrm{Min} /+}(\mathrm{n}=10)$ mice and their respective wild type controls, $\operatorname{Mstn}^{+/+}:: \mathrm{Apc}^{+/+}(\mathrm{n}=6), \mathrm{Mstn}^{+/-}:: \mathrm{Apc}^{+/+}(\mathrm{n}=6)$, and $\mathrm{Mstn}^{-/}:: \mathrm{Apc}^{+/+}(\mathrm{n}=11)$ mice......

Figure 7.7.1 (Section 4.7, Figures 4.7.3A, 4.7.3B, and 4.7.3D): Immunoblots for Atrogin-1 and MuRF1.

Figure 7.7.2 (Section 4.7, Figures 4.7.3A, 4.7.3B, and 4.7.3D): Immunoblots for GAPDH corresponding to the Atrogin-1 and MuRF1 immunoblots represented in Figure 7.7.1............. 145

Figure 7.7.3 (Section 4.7, Figures 4.7.3A and 4.7.3B): Immunoblots for MuRF1 146 
Figure 7.7.4 (Section 4.7, Figures 4.7.3A and 4.7.3B): Immunoblots for GAPDH corresponding to the MuRF1 immunoblots represented in Figure 7.7.3.

Table 7.7.2 (Section 4.7, Figure 4.7.1): Tibia lengths for $\mathrm{Mstn}^{+/+}:: \mathrm{Apc}^{\mathrm{Min} /+}(\mathrm{n}=6)$, $\mathrm{Mstn}^{+/-}$ $:: \mathrm{Apc}^{\mathrm{Min} /+}(\mathrm{n}=4)$, and $\mathrm{Mstn}^{-/}:: \mathrm{Apc}^{\mathrm{Min} /+}(\mathrm{n}=10)$ mice and their respective wild type controls, $\mathrm{Mstn}^{+/+}:: \mathrm{Apc}^{+/+}(\mathrm{n}=6), \mathrm{Mstn}^{+/}:: \mathrm{Apc}^{+/+}(\mathrm{n}=6)$, and $\mathrm{Mstn}^{-/}:: \mathrm{Apc}^{+/+}(\mathrm{n}=11)$ mice.

7.8 The deletion of myostatin did not alter the intestinal tumor biology of the $\mathrm{Apc}^{\mathrm{Min} /+}$ mouse

Figure 7.8.1 (Section 4.8, Figure 4.8.1): Sacrificed body weights and spleen weights from 20 to 21 week old $\mathrm{Mstn}^{+/+}:: \mathrm{Apc}^{\mathrm{Min} /+}, \mathrm{Mstn}^{+/}:: \mathrm{Apc}^{\mathrm{Min} /+}$, and $\mathrm{Mstn}^{-/-}: \mathrm{Apc}^{\mathrm{Min} /+}$ mice.

Figure 7.8.2 (Section 4.8, Figure 4.8.1B): Small intestine tumor diameter for $\mathrm{Mstn}^{+++}:: \mathrm{Apc}^{\mathrm{Min} /+}$, $\mathrm{Mstn}^{+/}:: \mathrm{Apc}^{\mathrm{Min} /+}$, and $\mathrm{Mstn}^{-/}:: \mathrm{Apc}^{\mathrm{Min} /+}$ mice. 150

Table 7.8.1 (Section 4.8, Figure 4.8.1A and Figure 4.8.1B): Small intestine tumor number and diameter for $\mathrm{Mstn}^{+/+}:: \mathrm{Apc}^{\mathrm{Min} /+}, \mathrm{Mstn}^{+/}:: \mathrm{Apc}^{\mathrm{Min} /+}$, and $\mathrm{Mstn}^{-/-}:: \mathrm{Apc}^{\mathrm{Min} /+}$ mice. ............................. 151

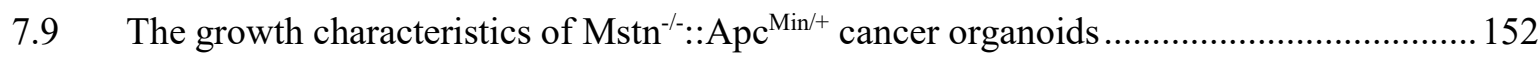

Figure 7.9.1 (Section 4.9, Figure 4.9.1B): Immunoblot for Cleaved Caspase-3 ........................... 152

Figure 7.9.2 (Section 4.9, Figures 4.9.1B and 4.9.1C): Immunoblot for GAPDH for the Cleaved

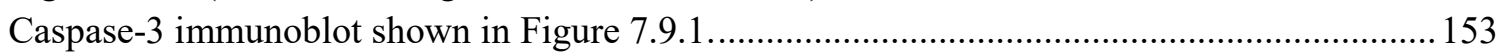

7.10 $\mathrm{Mstn}^{-/}$mice were resistant to colitis-induced colorectal carcinogenesis.............................. 154

Figure 7.10.1 (Section 4.10, Figures 4.10.2A-C): Images of 10\% NBF fixed $\mathrm{Mstn}^{+/+}$and $\mathrm{Mstn}^{-/-}$ colons used for macroscopic tumor analysis.

Figure 7.10.2 (Section 4.10, Figures 4.10.2A-C): Images of 10\% NBF fixed $\mathrm{Mstn}^{+/+}$and $\mathrm{Mstn}^{-/-}$ colons used for macroscopic tumor analysis.

Table 7.10.1 (Section 4.10, Figure 4.10.1E): Skeletal muscle weights for $\mathrm{Mstn}^{+/+}(\mathrm{n}=8)$ and $\mathrm{Mstn}^{-}$ /. $(\mathrm{n}=9)$ mice subjected to AOM/DSS model of colitis-induced colorectal carcinogenesis. ........ 156

Table 7.10.2 (Section 4.10, Figures 4.10.1 and 4.10.2): Tibia lengths for $\mathrm{Mstn}^{+/+}(\mathrm{n}=8)$ and $\mathrm{Mstn}^{-}$ /. $(n=9)$ mice subjected to AOM/DSS model of colitis-induced colorectal carcinogenesis. ........ 157

7.11 The intestinal biology of the $\mathrm{Mstn}^{-/-}$mice 158

Figure 7.11.1 (Section 4.11, Figure 4.11.1): Intestinal biology of $\mathrm{Mstn}^{+/+}:: \mathrm{Apc}^{\mathrm{Min} /+}, \mathrm{Mstn}^{+/-}$

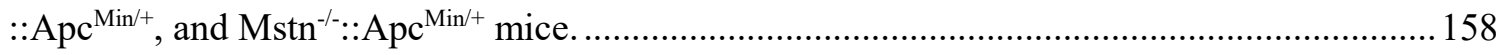

7.12 The role of myostatin in cancer cachexia.

Figure 7.12.1 (Section 5.2): Myostatin protein expression levels in cachectic $\mathrm{Apc}^{\mathrm{Min} /+}$ mice were inconclusive.

Figure 7.12.2 (Section 5.2): Myostatin serum levels in cachectic $\mathrm{Apc}^{\mathrm{Min} /+}$ mice were inconclusive.

Figure 7.12.3 (Section 5.2, Figure 7.12.1): Immunoblot for Myostatin (R\&D Systems).

Figure 7.12.4 (Section 5.2, Figures 7.12.1 and 7.12.3): Ponceau S for Myostatin (R\&D Systems) immunoblot in Figure 7.12.1 and Figure 7.12.3.

Figure 7.12.5 (Section 5.2, Figure 7.12.1): Immunoblot for Myostatin (Abcam).

Figure 7.12.6 (Section 5.2, Figures 7.12.1 and 7.12.5): Ponceau S for Myostatin (Abcam) immunoblot in Figure 7.12.1 and Figure 7.12.5. 
Figure 7.12.7 (Section 5.2, Figure 7.12.2A): Immunoblot for Myostatin (R\&D) (Figure 7.12.2A).

Figure 7.12.8 (Section 5.2, Figures 7.12.2A and 7.12.7): Ponceau S for Myostatin (R\&D) immunoblot in Figure 7.12.2A and Figure 7.12.7.

7.13 Murine background and intestinal tumorigenesis

Figure 7.13.1 (Section 5.5): Colony control $\mathrm{Mstn}^{+/+}:: \mathrm{Apc}^{\mathrm{Min} /+}$ mice displayed an increase in small intestine tumor multiplicity and tumor size compared to $\mathrm{Mstn}^{+++}:: \mathrm{Apc}^{\mathrm{Min} /+(\mathrm{Het})}$ mice.

Figure 7.13.2 (Section 5.5, Figure 7.13.1): Sacrificed body weights and spleen weights for colony control $\mathrm{Mstn}^{+/+}:: \mathrm{Apc}^{\mathrm{Min} /+}$ and $\mathrm{Mstn}^{+/+}: \mathrm{Apc}^{\mathrm{Min} /+(\mathrm{Het})}$ mice. 168

Figure 7.13.3 (Section 5.5): Body weights, skeletal muscle weights, and spleen weights for colony control $\mathrm{Mstn}^{+/+}:: \mathrm{Apc}^{+/+}$and $\mathrm{Mstn}^{+/+}:: \mathrm{Apc}^{+/+}(\mathrm{Het})$ mice, and $\mathrm{Mstn}^{+++}:: \mathrm{Apc}^{++(\text {Het })}$ and $\mathrm{Mstn}^{+/+}:: \mathrm{Apc}^{\mathrm{Min} /+(\mathrm{Het})}$ mice.

Table 7.13.1 (Section 5.5, Figure 7.13.3E): Skeletal muscle weights for colony control $\mathrm{Mstn}^{+/+}:: \mathrm{Apc}^{\mathrm{Min} /+}(\mathrm{n}=26)$ and $\mathrm{Mstn}^{+/+}:: \mathrm{Apc}^{\mathrm{Min} /+}(\mathrm{Het})(\mathrm{n}=8)$ mice.

Table 7.13.2 (Section 5.5, Figures 7.13.3D-F): Tibia length ( $\mathrm{mm}$ ) for colony control $\mathrm{Mstn}^{+/+}:: \mathrm{Apc}^{\mathrm{Min} /+}(\mathrm{n}=10)$ and $\mathrm{Mstn}^{+/+}:: \mathrm{Apc}^{\mathrm{Min} /+}(\mathrm{n}=9)$ mice.

Table 7.13.3 (Section 5.5, Figure 7.13.3B): Skeletal muscle weights for colony control

$\mathrm{Mstn}^{+/+}:: \mathrm{Apc}^{+/+}$mice $(\mathrm{n}=11)$ and $\mathrm{Mstn}^{+/+}:: \mathrm{Apc}^{+/+}(\mathrm{Het})(\mathrm{n}=12)$ mice.

Table 7.13.4 (Section 5.5, Figure 7.13.3A-C): Tibia length ( $\mathrm{mm}$ ) for colony control

$\mathrm{Mstn}^{+/+}:: \mathrm{Apc}^{+/+}(\mathrm{n}=10)$ and $\mathrm{Mstn}^{+/+}:: \mathrm{Apc}^{+/+}(\mathrm{Het})(\mathrm{n}=14)$ mice.

Figure 7.13.4 (Section 5.5): Colony control $\mathrm{Mstn}^{+/+}$and $\mathrm{Mstn}^{+/+(H e t)}$ mice intestinal lengths and weights.

Figure 7.13.5 (Section 5.5): Growth curves and skeletal muscle weights of $\mathrm{Mstn}^{+/+}:: \mathrm{Apc}^{\mathrm{Min} /+}$, $\mathrm{Mstn}^{+/}:: \mathrm{Apc}^{\mathrm{Min} /+}$, and $\mathrm{Mstn}^{-/}:: \mathrm{Apc}^{\mathrm{Min} /+}$ mice and their respective controls.

Figure 7.13.6 (Section 5.5): Growth curves and skeletal muscle weights of $\mathrm{Mstn}^{+/+}:: \mathrm{Apc}^{\mathrm{Min} /+}$, $\mathrm{Mstn}^{+/-}:: \mathrm{Apc}^{\mathrm{Min} /+}$, and $\mathrm{Mstn}^{-/}:: \mathrm{Apc}^{\mathrm{Min} /+}$ mice and their respective controls.

Table 7.13.5 (Section 5.5, Figure 7.13.6A): Skeletal muscle weights for $\mathrm{Mstn}^{+++}:: \mathrm{Apc}^{\mathrm{Min} /+}(\mathrm{n}=8)$,

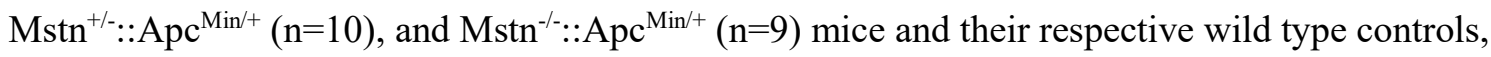
$\operatorname{Mstn}^{+/+}: \mathrm{Apc}^{+/+}(\mathrm{n}=12), \mathrm{Mstn}^{+/}:: \mathrm{Apc}^{+/+}(\mathrm{n}=10)$, and $\mathrm{Mstn}^{-/}:: \mathrm{Apc}^{+/+}(\mathrm{n}=10)$ mice. 177

Table 7.13.6 (Section 5.5, Figures 7.13.5 and 7.13.6): Tibia lengths for $\operatorname{Mstn}^{+/+}:: \mathrm{Apc}^{\mathrm{Min} /+}(\mathrm{n}=9)$, $\mathrm{Mstn}^{+/}:: \mathrm{Apc}^{\mathrm{Min} /+}(\mathrm{n}=13)$, and $\mathrm{Mstn}^{-/}:: \mathrm{Apc}^{\mathrm{Min} /+}(\mathrm{n}=9)$ mice and their respective wild type controls, $\operatorname{Mstn}^{+/+}:: \mathrm{Apc}^{+/+}(\mathrm{n}=14), \mathrm{Mstn}^{+/}:: \mathrm{Apc}^{+/+}(\mathrm{n}=11)$, and $\mathrm{Mstn}^{-/}:: \mathrm{Apc}^{+/+}(\mathrm{n}=11)$ mice. 178

Figure 7.13.7 (Section 5.5): The deletion of myostatin suppressed the intestinal tumor number and diameter in 18 week old $\mathrm{Apc}^{\mathrm{Min} /+}$ mice.

8. References 



\section{List of Figures}

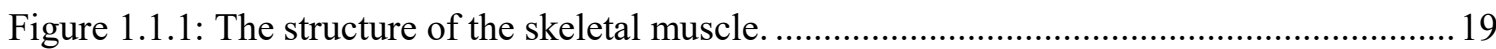

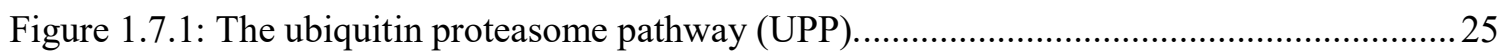

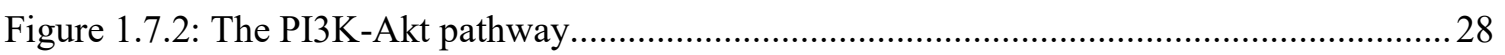

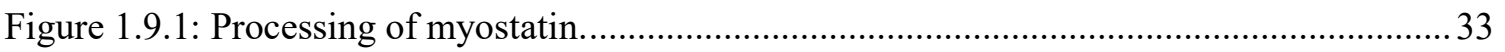

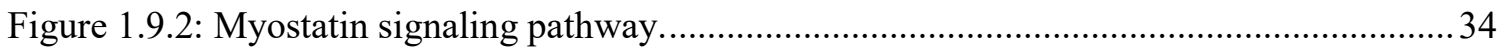

Figure 4.1.1: Aging male $\mathrm{Apc}^{\mathrm{Min} /+}$ mice displayed body weight loss.............................................. 66

Figure 4.2.1: Cachectic $\mathrm{Apc}^{\mathrm{Min} /+}$ mice demonstrated skeletal muscle atrophy. .............................70

Figure 4.2.2: Cachectic $\mathrm{Apc}^{\mathrm{Min} /+}$ mice exhibited adipose tissue atrophy. ....................................71

Figure 4.3.1: Increased levels of Atrogin-1 and Murfl mRNA was associated with increasing severity of cancer cachexia in the $\mathrm{Apc}^{\mathrm{Min} /+}$ mouse.

Figure 4.3.2: Elevated protein expression of key mediators of the ubiquitin-proteasome pathway was associated with increasing severity of cancer cachexia in the $\mathrm{Apc}^{\mathrm{Min} /+}$ mouse

Figure 4.4.1: Myostatin gene expression levels were elevated in the GAS muscles of moderately

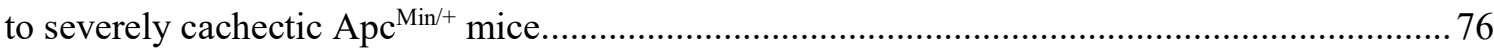

Figure 4.5.1: Small intestine polyps from $\mathrm{Apc}^{\mathrm{Min} /+}$ mice express IL-6 and TNF $\alpha \ldots \ldots \ldots \ldots \ldots \ldots \ldots \ldots . . . . . . . . . . . .78$

Figure 4.5.2: Small intestine polyp number did not correlate with male $\mathrm{Apc}^{\mathrm{Min} /+}$ body weight or

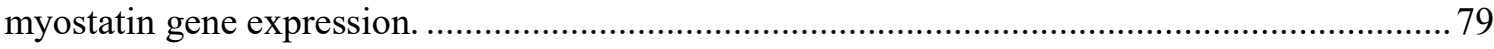

Figure 4.6.1: The loss of body mass due to cancer cachexia in male $\mathrm{Apc}^{\mathrm{Min}+}$ mice was not prevented by sActRIIB treatment.

Figure 4.6.2: Soluble ActRIIB treatment of male $\mathrm{Apc}^{\mathrm{Min} /+}$ mice at 16 weeks partially prevented skeletal muscle atrophy and improved forelimb grip strength.

Figure 4.6.3: Soluble ActRIIB treatment of male $\mathrm{Apc}^{\mathrm{Min} /+}$ mice at 16 weeks failed to improve the TA myofiber cross-sectional area.

Figure 4.6.4: Soluble ActRIIB treatment of pre-cachectic male $\mathrm{Apc}^{\mathrm{Min} /+}$ mice failed to prevent the onset of cancer cachexia.

Figure 4.6.5: Soluble ActRIIB treatment of pre-cachectic male $\mathrm{Apc}^{\mathrm{Min} /+}$ mice partially improved forelimb grip strength.

Figure 4.6.6: The small intestine tumor biology of $\mathrm{Apc} \mathrm{Min}^{\mathrm{M} /+}$ mice administered with sActRIIB from 16 weeks of age.

Figure 4.7.1: Genetic ablation of myostatin prevented the onset of cancer cachexia in 18 week old $\mathrm{Apc}^{\mathrm{Min} /+}$ mice.

Figure 4.7.2: TA Myofiber cross-sectional area of 18 week old $\mathrm{Mstn}^{+/}:: \mathrm{Apc}^{\mathrm{Min} /+}$ and $\mathrm{Mstn}^{-/}$ $:: \mathrm{Apc}^{\mathrm{Min} /+}$ mice...

Figure 4.7.3: The absence of myostatin suppressed the over-expression of the ubiquitin proteasome pathway in skeletal muscles of 18 week old $\mathrm{Mstn}^{+/}:: \mathrm{Apc}^{\mathrm{Min} /+}$ and $\mathrm{Mstn}^{-/}:: \mathrm{Apc}^{\mathrm{Min} /+}$ mice.

Figure 4.7.4: Genetic ablation of myostatin preserved adipose tissue and spleen mass in 18 week old $\mathrm{Apc}^{\mathrm{Min} /+}$ mice 
Figure 4.8.1: Genetic ablation of myostatin failed to alter the tumor biology of the $\mathrm{Apc}^{\mathrm{Min} /+}$ mouse.

Figure 4.9.1: The growth characteristics of $\mathrm{Mstn}^{+++}:: \mathrm{Apc}^{\mathrm{Min} /+}, \mathrm{Mstn}^{+/}:: \mathrm{Apc}^{\mathrm{Min} /+}$, and $\mathrm{Mstn}^{-/-}$ $:: \mathrm{Apc}^{\mathrm{Min} /+}$ small intestine cancer organoids.

Figure 4.10.1: Body weights for male $\mathrm{Mstn}^{+/+}$and $\mathrm{Mstn}^{-/-}$mice subjected to the AOM/DSS model

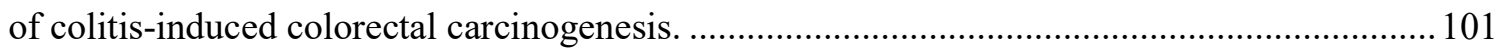

Figure 4.10.2: Absence of myostatin suppressed colitis-induced colorectal tumorigenesis....... 102

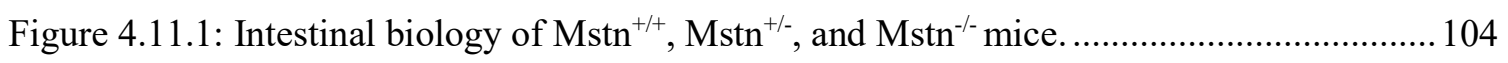

Figure 5.6.1: Investigating the molecular mechanism of myostatin and cancer.......................... 116

Figure 7.3.1 (Section 4.3, Figure 4.3.2A and 4.3.2B): Immunoblot for Atrogin-1.................... 123

Figure 7.3.2 (Section 4.3, Figures 4.3.2A and 4.3.2B): Immunoblot for p-FoxO3a................... 124

Figure 7.3.3 (Section 4.3): Immunoblot for GAPDH corresponding to Atrogin-1 and p-FoxO3a immunoblots represented in Figure 7.3.1 and Figure 7.3.2 (Section 4.3, Figure 4.3.2A and 4.3.2B)

Figure 7.3.4 (Section 4.3, Figures 4.3.2A and 4.3.2B): Immunoblot for FoxO3a immunoblots.126

Figure 7.3.5: Immunoblot for GAPDH corresponding to FoxO3a immunoblots represented in

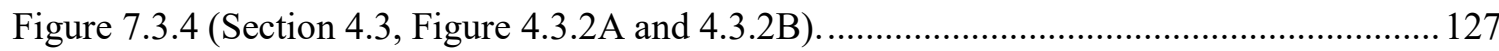

Figure 7.3.6 (Section 4.3, Figures 4.3.2A and 4.3.2B): Immunoblot for MuRF1...................... 128

Figure 7.3.7: Immunoblot for GAPDH corresponding to MuRF1 immunoblots represented in

Figure 7.3.6 (Section 4.3, Figures 4.3.2A and 4.3.2B). .

Figure 7.4.1 (Section 4.4, Figure 4.4.1): Myostatin gene expression levels were elevated in the GAS muscles of moderately to severely cachectic $\mathrm{Apc}^{\mathrm{Min} /+}$ mice.

Figure 7.4.2 (Section 4.4, Figure 4.4.1): Myostatin gene expression levels in the GAS muscles of the cachectic $\mathrm{Apc}^{\mathrm{Min} /+}$ mice.

Figure 7.5.1 (Section 4.5, Figure 4.5.1): Small intestine polyps from $\mathrm{Apc}^{\mathrm{Min} /+}$ male mice express interleukin- 6 and $\mathrm{TNF} \alpha$.

Figure 7.5.2 (Section 4.5, Figure 4.5.1): Small intestine polyps from $\mathrm{Apc}^{\mathrm{Min} /+}$ male mice do not express TNF $\alpha$ variant 2

Figure 7.6.1 (Section 4.6, Figure 4.6.3): Soluble ActRIIB treatment of male $\mathrm{Apc}^{\mathrm{Min} /+}$ mice at 16 weeks failed to improve the TA myofiber cross-sectional area.

Figure 7.7.1 (Section 4.7, Figures 4.7.3A, 4.7.3B, and 4.7.3D): Immunoblots for Atrogin-1 and MuRF1.

Figure 7.7.2 (Section 4.7, Figures 4.7.3A, 4.7.3B, and 4.7.3D): Immunoblots for GAPDH corresponding to the Atrogin-1 and MuRF1 immunoblots represented in Figure 7.7.1.

Figure 7.7.3 (Section 4.7, Figures 4.7.3A and 4.7.3B): Immunoblots for MuRF1 146

Figure 7.7.4 (Section 4.7, Figures 4.7.3A and 4.7.3B): Immunoblots for GAPDH corresponding to the MuRF1 immunoblots represented in Figure 7.7.3.

Figure 7.8.1 (Section 4.8, Figure 4.8.1): Sacrificed body weights and spleen weights from 20 to

21 week old $\mathrm{Mstn}^{+/+}:: \mathrm{Apc}^{\mathrm{Min} /+}, \mathrm{Mstn}^{+/}:: \mathrm{Apc}^{\mathrm{Min} /+}$, and $\mathrm{Mstn}^{-/-}: \mathrm{Apc}^{\mathrm{Min} /+}$ mice. 149 
Figure 7.8.2 (Section 4.8, Figure 4.8.1B): Small intestine tumor diameter for $\mathrm{Mstn}^{+/+}:: \mathrm{Apc}^{\mathrm{Min} /+}$,

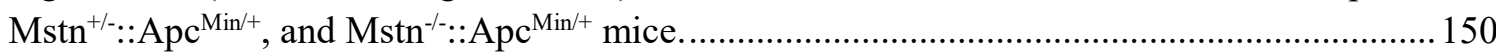

Figure 7.9.1 (Section 4.9, Figure 4.9.1B): Immunoblot for Cleaved Caspase-3 152

Figure 7.9.2 (Section 4.9, Figures 4.9.1B and 4.9.1C): Immunoblot for GAPDH for the Cleaved Caspase-3 immunoblot shown in Figure 7.9.1.

Figure 7.10.1 (Section 4.10, Figures 4.10.2A-C): Images of 10\% NBF fixed $\mathrm{Mstn}^{+/+}$and $\mathrm{Mstn}^{-/-}$ colons used for macroscopic tumor analysis.

Figure 7.10.2 (Section 4.10, Figures 4.10.2A-C): Images of 10\% NBF fixed $\mathrm{Mstn}^{+/+}$and $\mathrm{Mstn}^{-/-}$ colons used for macroscopic tumor analysis.

Figure 7.11.1 (Section 4.11, Figure 4.11.1): Intestinal biology of $\mathrm{Mstn}^{++}:: \mathrm{Apc}^{\mathrm{Min} /+}, \mathrm{Mstn}^{+/-}$

$:: \mathrm{Apc}^{\mathrm{Min} /+}$, and $\mathrm{Mstn}^{-/}:: \mathrm{Apc}^{\mathrm{Min} /+}$ mice.

Figure 7.12.1 (Section 5.2): Myostatin protein expression levels in cachectic $\mathrm{Apc}^{\mathrm{Min} /+}$ mice were inconclusive.

Figure 7.12.2 (Section 5.2): Myostatin serum levels in cachectic $\mathrm{Apc}^{\mathrm{Min} /+}$ mice were inconclusive.

Figure 7.12.3 (Section 5.2, Figure 7.12.1): Immunoblot for Myostatin (R\&D Systems). 161

Figure 7.12.4 (Section 5.2, Figures 7.12.1 and 7.12.3): Ponceau S for Myostatin (R\&D Systems) immunoblot in Figure 7.12.1 and Figure 7.12.3.

Figure 7.12.5 (Section 5.2, Figure 7.12.1): Immunoblot for Myostatin (Abcam).

Figure 7.12.6 (Section 5.2, Figures 7.12.1 and 7.12.5): Ponceau S for Myostatin (Abcam) immunoblot in Figure 7.12.1 and Figure 7.12.5.

Figure 7.12.7 (Section 5.2, Figure 7.12.2A): Immunoblot for Myostatin (R\&D) (Figure 7.12.2A).

Figure 7.12.8 (Section 5.2, Figures 7.12.2A and 7.12.7): Ponceau S for Myostatin (R\&D) immunoblot in Figure 7.12.2A and Figure 7.12.7 166

Figure 7.13.1 (Section 5.5): Colony control $\mathrm{Mstn}^{+/+}:: \mathrm{Apc}^{\mathrm{Min} /+}$ mice displayed an increase in small intestine tumor multiplicity and tumor size compared to $\mathrm{Mstn}^{+/+}:: \mathrm{Apc}^{\mathrm{Min} /+(\mathrm{Het})}$ mice.

Figure 7.13.2 (Section 5.5, Figure 7.13.1): Sacrificed body weights and spleen weights for colony control Mstn ${ }^{+/+}:: \mathrm{Apc}^{\mathrm{Min} /+}$ and $\mathrm{Mstn}^{+/+}:: \mathrm{Apc}^{\mathrm{Min} /+(\mathrm{Het})}$ mice.

Figure 7.13.3 (Section 5.5): Body weights, skeletal muscle weights, and spleen weights for colony control $\mathrm{Mstn}^{+/+}:: \mathrm{Apc}^{+/+}$and $\mathrm{Mstn}^{+/+}:: \mathrm{Apc}^{+/+}(\mathrm{Het})$ mice, and $\mathrm{Mstn}^{+/+}:: \mathrm{Apc}^{+/+(\mathrm{Het})}$ and $\mathrm{Mstn}^{+/+}:: \mathrm{Apc}^{\mathrm{Min} /+(\mathrm{Hett})}$ mice.

Figure 7.13.4 (Section 5.5): Colony control $\mathrm{Mstn}^{+/+}$and $\mathrm{Mstn}^{+/+(H e t)}$ mice intestinal lengths and weights.

Figure 7.13.5 (Section 5.5): Growth curves and skeletal muscle weights of $\mathrm{Mstn}^{+/+}:: \mathrm{Apc}^{\mathrm{Min} /+}$, $\mathrm{Mstn}^{+/}:: \mathrm{Apc}^{\mathrm{Min} /+}$, and $\mathrm{Mstn}^{-/}:: \mathrm{Apc}^{\mathrm{Min} /+}$ mice and their respective controls.

Figure 7.13.6 (Section 5.5): Growth curves and skeletal muscle weights of $\mathrm{Mstn}^{+/+}:: \mathrm{Apc}^{\mathrm{Min} /+}$, $\mathrm{Mstn}^{+/-}:: \mathrm{Apc}^{\mathrm{Min} /+}$, and $\mathrm{Mstn}^{-/}:: \mathrm{Apc}^{\mathrm{Min} /+}$ mice and their respective controls.

Figure 7.13.7 (Section 5.5): The deletion of myostatin suppressed the intestinal tumor number and diameter in 18 week old $\mathrm{Apc}^{\mathrm{Min} /+}$ mice. 


\section{List of Tables}

Table 3.1: Antibodies and the immunoblotting conditions.

Table 3.2: Primers.

Table 4.1.1: Body weights of $\mathrm{Apc}^{\mathrm{Min} /+}$ mice stratified by their percentage body weight loss.

Table 7.1.1: Body weights for male $\mathrm{Apc}^{\mathrm{Min} /+}$ mice ( $\left.\mathrm{n}=9-50\right)$ and wild type controls $(\mathrm{n}=11-38)$ measured from 5 to 20 weeks.

Table 7.1.2: Male $\mathrm{Apc}^{\mathrm{Min} /+}$ mice used for the cancer cachexia stratification trial

Table 7.1.3: Tibia lengths $(\mathrm{mm})$ for wild type $(\mathrm{n}=6)$, non-cachectic $(\mathrm{n}=7)$, early $(\mathrm{n}=5)$, intermediate $(\mathrm{n}=4)$, and severe $(\mathrm{n}=6)$ cachectic $\mathrm{Apc}^{\mathrm{Min} /+}$ mice.

Table 7.2.1: Skeletal muscle weights of $\mathrm{Apc}^{\mathrm{Min} /+}$ mice stratified by percentage body weight loss.

Table 7.6.1: Dosage of sActRIIB administered to $\mathrm{Apc}^{\mathrm{Min} /+}$ mice at 16 weeks.

Table 7.6.2: Grip strength, measured in Newtons $(\mathrm{N})$, for age-matched wild type $(\mathrm{n}=7)$, dialysis buffer $(\mathrm{n}=11)$, and sActRIIB treated $\mathrm{Apc}^{\mathrm{Min} /+}(\mathrm{n}=9)$ mice.

Table 7.6.3: Skeletal muscle weights for dialysis buffer $(\mathrm{n}=11)$ and sActRIIB treated Apc ${ }^{\mathrm{Min} /+}$ $(\mathrm{n}=9)$ mice.

Table 7.6.4: Skeletal muscle weights for dialysis buffer $(\mathrm{n}=8)$ and sActRIIB treated Apc $\mathrm{Min}^{\mathrm{H}}$ $(\mathrm{n}=9)$ mice.

Table 7.6.5: Tibia length $(\mathrm{mm})$ for dialysis buffer $(\mathrm{n}=11)$ and sActRIIB treated $\mathrm{Apc}^{\mathrm{Min} /+}(\mathrm{n}=9)$ mice.

Table 7.6.6: Dosage of sActRIIB administered to $\mathrm{Apc}^{\mathrm{Min} /+}$ mice at 14 weeks.

Table 7.6.7: Skeletal muscle weights for dialysis buffer $(\mathrm{n}=6)$ and sActRIIB treated $\mathrm{Apc}^{\mathrm{Min} /+}$ $(\mathrm{n}=5)$ mice.

Table 7.6.8: Tibia length $(\mathrm{mm})$ for dialysis buffer $(\mathrm{n}=6)$ and $\mathrm{sActRIIB}$ treated $\mathrm{Apc}^{\mathrm{Min} /+}(\mathrm{n}=5)$ mice.

Table 7.7.1: Skeletal muscle weights for $\operatorname{Mstn}^{+/+}:: \mathrm{Apc}^{\mathrm{Min} /+}(\mathrm{n}=6), \mathrm{Mstn}^{+/}:: \mathrm{Apc}^{\mathrm{Min} /+}(\mathrm{n}=4)$, and $\mathrm{Mstn}^{-/}:: \mathrm{Apc}^{\mathrm{Min} /+}(\mathrm{n}=10)$ mice and their respective wild type controls, $\mathrm{Mstn}^{+/+}:: \mathrm{Apc}^{+/+}(\mathrm{n}=6)$, $\mathrm{Mstn}^{+/}:: \mathrm{Apc}^{+/+}(\mathrm{n}=6)$, and $\mathrm{Mstn}^{-/}:: \mathrm{Apc}^{+/+}(\mathrm{n}=11)$ mice......

Table 7.7.2: Tibia lengths for $\mathrm{Mstn}^{+/+}:: \mathrm{Apc}^{\mathrm{Min} /+}(\mathrm{n}=6), \mathrm{Mstn}^{+/-}:: \mathrm{Apc}^{\mathrm{Min} /+}(\mathrm{n}=4)$, and $\mathrm{Mstn}^{-/-}$ $:: \mathrm{Apc}^{\mathrm{Min} /+}(\mathrm{n}=10)$ mice and their respective wild type controls, $\mathrm{Mstn}^{+/+}:: \mathrm{Apc}^{+/+}(\mathrm{n}=6), \mathrm{Mstn}^{+/-}$ $:: \mathrm{Apc}^{+/+}(\mathrm{n}=6)$, and $\mathrm{Mstn}^{-/}:: \mathrm{Apc}^{+/+}(\mathrm{n}=11)$ mice.

Table 7.8.1 (Section 4.8, Figure 4.8.1A and Figure 4.8.1B): Small intestine tumor number and diameter for Mstn+/+::ApcMin/+, Mstn+/-::ApcMin/+, and Mstn-/-::ApcMin/+ mice.

Table 7.10.1: Skeletal muscle weights for $\operatorname{Mstn}^{+/+}(n=8)$ and $\operatorname{Mstn}^{-/-}(n=9)$ mice subjected to AOM/DSS model of colitis-induced colorectal carcinogenesis.

Table 7.10.2: Tibia lengths for $\mathrm{Mstn}^{+/+}(\mathrm{n}=8)$ and $\mathrm{Mstn}^{-/-}(\mathrm{n}=9)$ mice subjected to AOM/DSS model of colitis-induced colorectal carcinogenesis.

Table 7.13.1: Skeletal muscle weights for colony control $\mathrm{Mstn}^{+/+}:: \mathrm{Apc}^{\mathrm{Min} /+}(\mathrm{n}=26)$ and $\mathrm{Mstn}^{+/+}:: \mathrm{Apc}^{\mathrm{Min} /+}(\mathrm{Het})(\mathrm{n}=8)$ mice. 
Table 7.13.2: Tibia length (mm) for colony control $\mathrm{Mstn}^{+/+}:: \mathrm{Apc}^{\mathrm{Min} /+}(\mathrm{n}=10)$ and $\mathrm{Mstn}^{+/+}:: \mathrm{Apc}^{\mathrm{Min} /+}(\mathrm{n}=9)$ mice.

Table 7.13.3: Skeletal muscle weights for colony control $\mathrm{Mstn}^{+/+}:: \mathrm{Apc}^{+/+}$mice $(\mathrm{n}=11)$ and $\mathrm{Mstn}^{+/+}:: \mathrm{Apc}^{+/+(\mathrm{Het})}(\mathrm{n}=12)$ mice.

Table 7.13.4: Tibia length (mm) for colony control $\mathrm{Mstn}^{+/+}:: \mathrm{Apc}^{+/+}(\mathrm{n}=10)$ and $\mathrm{Mstn}^{+/+}:: \mathrm{Apc}^{+/+}$ (Het) $(\mathrm{n}=14)$ mice.

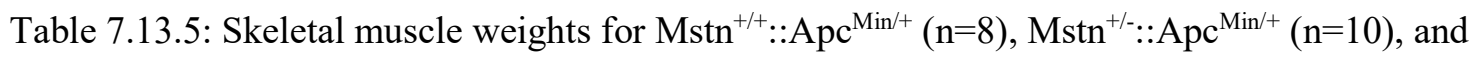
$\mathrm{Mstn}^{-/-}:: \mathrm{Apc}^{\mathrm{Min} /+}(\mathrm{n}=9)$ mice and their respective wild type controls, $\mathrm{Mstn}^{+/+}:: \mathrm{Apc}^{+/+}(\mathrm{n}=12)$, $\mathrm{Mstn}^{+/}: \because \mathrm{Apc}^{+/+}(\mathrm{n}=10)$, and $\mathrm{Mstn}^{-/}:: \mathrm{Apc}^{+/+}(\mathrm{n}=10)$ mice.

Table 7.13.6: Tibia lengths for $\mathrm{Mstn}^{+/+}:: \mathrm{Apc}^{\mathrm{Min} /+}(\mathrm{n}=9), \mathrm{Mstn}^{+/-}:: \mathrm{Apc}^{\mathrm{Min} /+}(\mathrm{n}=13)$, and $\mathrm{Mstn}^{-/-}$ $:: \mathrm{Apc}^{\mathrm{Min} /+}(\mathrm{n}=9)$ mice and their respective wild type controls, $\mathrm{Mstn}^{+/+}:: \mathrm{Apc}^{+/+}(\mathrm{n}=14), \mathrm{Mstn}^{+/-}$ $:: \mathrm{Apc}^{+/+}(\mathrm{n}=11)$, and $\mathrm{Mstn}^{-/}:: \mathrm{Apc}^{+/+}(\mathrm{n}=11)$ mice. 


\section{List of Abbreviations}

\begin{tabular}{|c|c|}
\hline$\left(\mathrm{NH}_{4}\right)_{2} \mathrm{SO}_{4}$ & Ammonium Sulfate \\
\hline 4E-BP1 & Eukaryotic Translation Initiation Factor 4E Binding Protein 1 \\
\hline 6-FAM & 6-Carboxyfluorescein \\
\hline AAV & Adeno-associated virus \\
\hline ActRIIB & Activin Receptor Type II B \\
\hline ACVR2 & Activin Receptor Type II B \\
\hline AIDS & Acquired Immune Deficiency Syndrome \\
\hline ALK4 & Activin Type 1B \\
\hline ALK5 & TGF- $\beta$ Receptor Type 1 \\
\hline ANOVA & Analysis of Variance \\
\hline $\mathrm{AOM}$ & Azoxymethane \\
\hline APC & Adenomatous Polyposis Coli \\
\hline ATP & Adenosine Triphosphate \\
\hline BMP & Bone Morphogenic Protein \\
\hline Bnip3 & BCL2/adenovirus E1B 19 kDa Protein-Interacting Protein 3 \\
\hline $\mathrm{Ca} 2+$ & Calcium \\
\hline $\mathrm{CaCl}_{2}$ & Calcium Chloride \\
\hline Cas9 & CRISPR Associated Protein 9 \\
\hline $\mathrm{CBCs}$ & Crypt Base Columnar Cells \\
\hline $\mathrm{CO}_{2}$ & Carbon Dioxide \\
\hline CRISPR & Clustered Regularly Interspaced Short Palindromic Repeats \\
\hline $\mathrm{CXC} 2 \mathrm{R}$ & C-X-C Motif Chemokine Receptor 2 \\
\hline DEPC & Diethylpyrocarbonate \\
\hline dMCK & Double Muscle Creatine Kinase \\
\hline DMEM & Dulbecco's Modified Eagle Medium \\
\hline DNA & Deoxyribonucleic Acid \\
\hline dNTP & Deoxynucleotide \\
\hline DSS & Dextran Sodium Sulfate \\
\hline EDL & Extensor Digitorum Longus \\
\hline EDTA & Ethylenediaminetetraacetic acid \\
\hline EGF & Epithelial Growth Factor \\
\hline eIF2B & Eukaryotic Translation Initiation Factor 2B \\
\hline eIF3-f & Elongation Initiation Factor 3 Subunit 5 \\
\hline EPI & Epididymal \\
\hline ER & Endoplasmic Reticulum \\
\hline FIP200 & Focal Adhesion Kinase Family Interacting Protein of $200 \mathrm{kDa}$ \\
\hline FoxO & Forkhead Box \\
\hline Gabarap1 & Gamma-Aminobutyric Acid Receptor-Associated Protein 1 \\
\hline GAP & GTPase Activating Protein \\
\hline GAS & Gastrocnemius \\
\hline GDF-11 & Growth and Differentiation Factor 11 \\
\hline GDF-8 & Growth and Differentiation Factor 8 \\
\hline
\end{tabular}




\begin{tabular}{|c|c|}
\hline GSK3 $\beta$ & Glycogen Synthase-3 Beta \\
\hline $\mathrm{H} \& \mathrm{E}$ & Hematoxylin and eosin \\
\hline $\mathrm{HCl}$ & Hydrochloric Acid \\
\hline HEPES & 4-(2-hydroxyethyl)-1-piperazineethanesulfonic acid \\
\hline HI-FBS & Heat Inactivated Fetal Bovine Serum \\
\hline IBD & Irritable Bowel Disease \\
\hline IEC & Intestinal Epithelial Cells \\
\hline IGF-1 & Insulin-like Growth Factor 1 \\
\hline IL-6 & Interleukin-6 \\
\hline ING & Inguinal \\
\hline IPTG & Isopropyl ß-D-1-thiogalactopyranoside \\
\hline IRS & Insulin Receptor Substrate \\
\hline JOE (NHS Ester) & 6-carboxy-4',5'-dichloro-2',7'-dimethoxyfluorescein \\
\hline $\mathrm{KCl}$ & Potassium Chloride \\
\hline $\mathrm{kDa}$ & Kilodaltons \\
\hline $\mathrm{KH}_{2} \mathrm{PO}_{4}$ & Monopotassium Phosphate \\
\hline LAMP2A & Lysosomal-Associated Membrane Protein 2A \\
\hline LAP & Latent Associated Peptide \\
\hline LC3 & Microtubule-associated Protein 1 Light Chain 3 \\
\hline LRC & Label-Retaining Cells \\
\hline MAFbx & Muscle Atrophy F box \\
\hline MAPK & Mitogen-Activated Protein Kinase \\
\hline $\mathrm{MgCl}_{2}$ & Magnesium Chloride \\
\hline $\mathrm{MgSO}_{4}$ & Magnesium Sulphate \\
\hline MHC & Myosin Heavy Chain \\
\hline Min & Multiple Intestinal Neoplasia \\
\hline MMR & Mismatch Repair \\
\hline MOM & Modifiers of the Min \\
\hline mRNA & Messenger Ribonucleic Acid \\
\hline$M_{s t n}^{-/-}$ & Myostatin Nullizygote \\
\hline mTOR & Mechanistic/Mammalian Target of Rapamycin \\
\hline mTORC1 & Mechanistic/Mammalian Target of Rapamycin Complex 1 \\
\hline MuRF1 & Muscle RING Finger 1 \\
\hline $\mathrm{Na}_{2} \mathrm{HPO}_{4}$ & Disodium Phosphate \\
\hline $\mathrm{NaCl}$ & Sodium Chloride \\
\hline $\mathrm{NaHCO}_{3}$ & Sodium Bicarbonate \\
\hline NBF & Neutral Buffered Formalin \\
\hline PACE & Pair Dibasic Amino-Acid Cleaving Enzymes \\
\hline PBS & Phosphate Buffered Saline \\
\hline PCR & Polymerase Chain Reaction \\
\hline PDK1 & Phosphoinositide-Dependent Kinase 1 \\
\hline PGC1- $\alpha$ & Peroxisome Proliferator-Activated Receptor- $\gamma$ Coactivator-1 Alpha \\
\hline PI3K & Phosphatidylinositol-3 Kinase \\
\hline
\end{tabular}




$\begin{array}{ll}\text { PIP2 } & \text { Phosphoinositide-4,5-Biphosphate } \\ \text { PIP3 } & \text { Phosphoinositide-3,4,5-Triphosphate } \\ \text { PMSF } & \text { Phenylmethane Sulfonyl Fluoride } \\ \text { QUAD } & \text { Quadriceps } \\ \text { Rb } & \text { Retinoblastoma } \\ \text { RETRO } & \text { Retroperitoneal } \\ \text { RHEB } & \text { Ras Homolog Enriched in Brain } \\ \text { RIPA } & \text { Radioimmunoprecipitation Assay Buffer } \\ \text { RMS } & \text { Rhabdomyosarcoma } \\ \text { S6K1 } & \text { S6 Kinase 1 } \\ \text { sActRIIB } & \text { Soluble ActRIIB } \\ \text { SDS } & \text { Sodium Dodecyl Sulfate } \\ \text { Sol } & \text { Soleus } \\ \text { TA } & \text { Tibialis Anterior } \\ \text { TA cells } & \text { Transit Amplifying Cells } \\ \text { TAE } & \text { Tris-Acetate-EDTA } \\ \text { TAK1 } & \text { TGF- } \beta \text { Activated Kinase } \\ \text { TBST } & \text { Tris Buffered Saline with Tween-20 } \\ \text { TGF- } \beta & \text { Transforming Growth Factor Beta } \\ \text { TNF } \alpha & \text { Tumor Necrosis Factor Alpha } \\ \text { TRAF6 } & \text { TNF Receptor Associated Factor 6 } \\ \text { TRIM32 } & \text { Tripartite Motif Containing 32 } \\ \text { TSC1/2 } & \text { Tuberous Sclerosis Complex 1 and 2 } \\ \text { UNK1 } & \text { Unc-51-Like Kinase 1 } \\ \text { UPP } & \text { Ubiquitin Proteasome Pathway } \\ \text { VPS } & \text { Vacuolar Sorting Protein }\end{array}$




\section{Abstract}

Myostatin is a TGF- $\beta$ superfamily member growth factor that regulates skeletal muscle mass and function. Although myostatin over-expression is associated with cancer-induced skeletal muscle wasting, also termed cancer cachexia, it is currently unknown whether this increase in myostatin levels can regulate tumorigenesis. We used the $A p c^{\mathrm{Min} /+}$ mouse to address this question. The $A p c^{\mathrm{Min} /+}$ mouse spontaneously develops multiple adenomas along the intestine. Using this model of intestinal tumorigenesis, we have demonstrated that the skeletal muscles of cachectic male $A p c^{\mathrm{Min} /+}$ mice express augmented amounts of myostatin mRNA. The inoculation of sActRIIB, an inhibitor of myostatin, partially prevented the loss of skeletal muscle mass and slightly improved the grip strength of cachectic $A p c^{\mathrm{Min} /+}$ mice when compared to dialysis buffer treated controls. In addition, the genetic inactivation of myostatin in the $\mathrm{Apc}^{\mathrm{Min} /+}$ mouse $\left(\mathrm{Msth}^{-/}: \because A p \mathrm{Cin}^{\mathrm{Mi+}}\right.$ ) delayed the symptoms of cancer cachexia, thus establishing myostatin as a potential modulator of muscle wasting. The intestinal tumor number in the Mstn ${ }^{{ }^{\prime}} \because: A p c^{\mathrm{Min} /+}$ mouse displayed no difference to the wild type control $\left(\mathrm{Mstn}^{+/+}: \because A p c^{\mathrm{Min} /+}\right)$. We next employed the AOM/DSS model of colorectal carcinogenesis to query the link between myostatin and intestinal carcinogenesis. The AOM/DSS model is a multistep chemical carcinogenesis protocol that induces the formation of several tumors in the colon. Unlike wild type $\mathrm{Mstn}^{+/+}$mice, $\mathrm{Mstn}^{-/-}$mice were resistant to AOM/DSS mediated colorectal carcinogenesis, as myostatin deficient mice demonstrated an approximately $75 \%$ reduction in the incidence of colonic tumors when compared to the wild type control. The findings in this thesis raise an argument for the development of therapeutics that block the function of myostatin to ameliorate cancer cachexia. Due to the disparity in the extent of colorectal tumorigenesis between the $\mathrm{Apc}^{\mathrm{Min} / \mathrm{+}}$ and AOM/DSS models, however, it is premature to suggest a definitive link between myostatin and intestinal tumorigenesis. 


\section{Introduction}

The skeletal muscle, a large organ that is 40 to $50 \%$ of the total body mass, is composed of highly organized myofibers that facilitate a range of voluntary movement such as jumping and running to finer actions such as typing or holding a glass [1]. The skeletal muscle also plays a central role in metabolism [2]. It is not only a repository for metabolites like glucose, fatty acids, and glutamine, but it also functions as an endocrine organ that secretes cytokines to modulate the metabolism of the whole body $[2,3]$. The skeletal muscle is susceptible to injury and degeneration due to direct trauma, genetic or neurological dysfunction, and pathological conditions such as cancer and diabetes $[4,5]$. If left untreated, the muscle would undergo atrophy and exhibit reduced functional capacity $[4,6]$. In some cases, chronic skeletal muscle atrophy may result in mortality [7]. The following section will describe the structure and function of the skeletal muscle.

\subsection{Skeletal muscle structure}

The skeletal muscle is one of three major muscle types found in the animal kingdom, the other two are cardiac and smooth muscle [1]. Each skeletal muscle is composed of numerous muscle fibers that run along the length of the muscle (Figure 1.1.1) [1]. These muscle fibers, or myofibers, are often found bundled together in groups of 10 to 100 to form a structure called the fascicle [1]. The fascicle is covered by a layer of connective tissue termed the perimysium [1]. Further examination of the muscle structure reveals that the myofibers themselves are encased by another connective tissue layer called the endomysium [1]. In addition to segregating each myofiber, the endomysium serves to house the nerves and capillaries [1]. The third connective tissue that encloses a muscle, called the epimysium, extends from the muscle, along with perimycium and endomysium, to form a tendon [1]. 


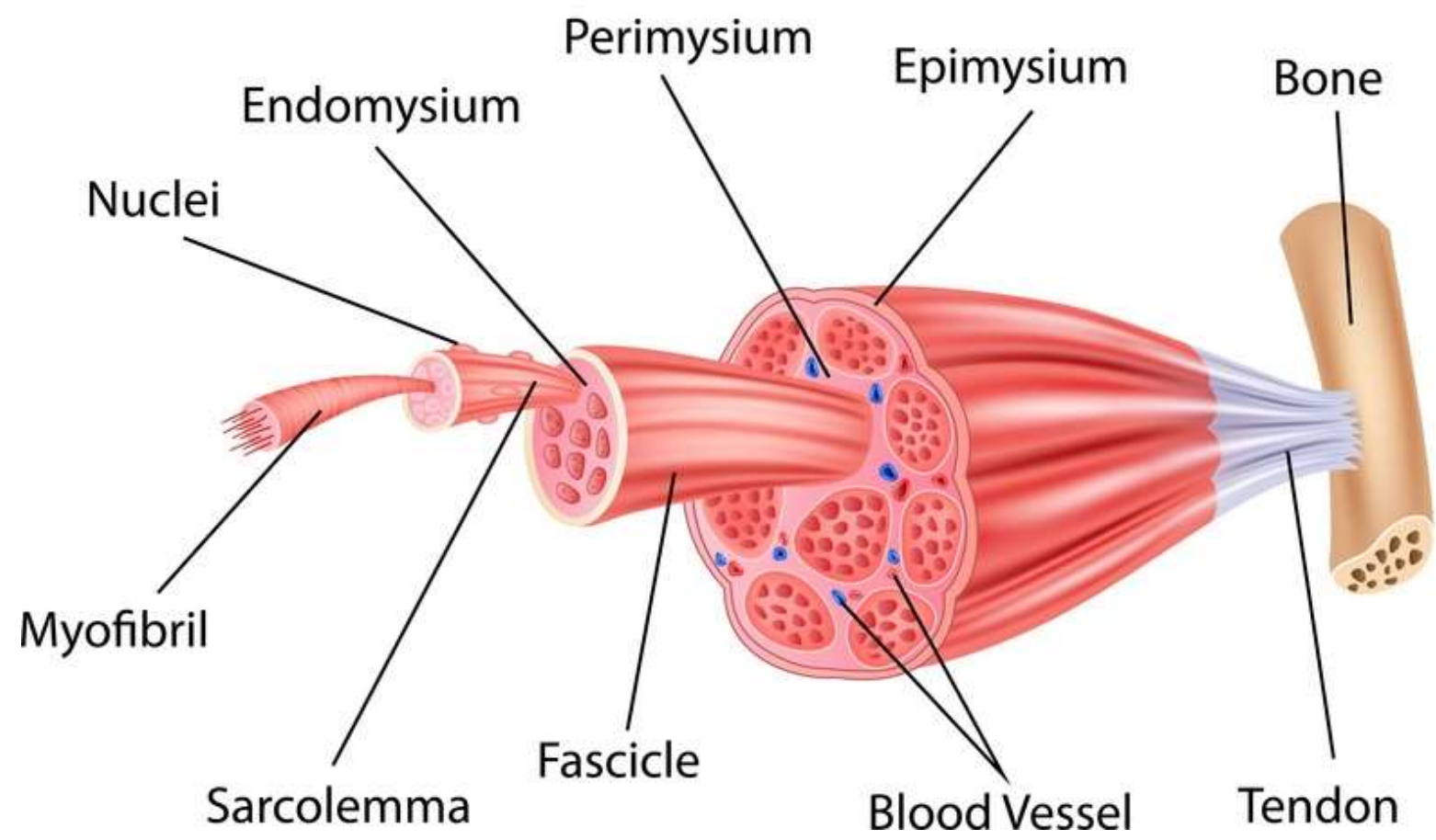

Figure 1.1.1: The structure of the skeletal muscle.

A schematic diagram representing the organization of the skeletal muscle. Image ID \#107548017, Author: tigatelu, Purchased from Fotolia.com.

\subsection{Structure of the myofiber}

The myofiber is a multinucleated, mitotically quiescent cell [1]. When examined longitudinally, the myofiber exhibits a striated pattern of dark and light bands. These characteristic striations within the myofiber represent the sarcomere [1]. The sarcomere is the functional unit for force generation [1]. These units of contraction contain two types of filaments [1]. The thick filament is mainly composed of myosin, whereas the thin filament is made up of actin [1]. The myosin molecule can be envisioned as two twisted golf clubs [8]. The globular heads of the myosin molecule contains ATPase activity which generates the force to facilitate muscle contraction [9]. The rod end, on the other hand, polymerizes with over 300 other myosin molecules to form the thick filament backbone [10]. Unlike myosin, actin is a globular protein that is polymerized as a helical filament [11]. The actin filament serves as a docking site for myosin crossbridge formation during muscle contraction [12]. The thin filament is also composed of two regulatory proteins, troponin and tropomyosin, which modulates the contraction process [13]. 
The length of the sarcomere is demarcated by two Z-discs. These zig-zag structures contain proteins which tether the thin filament $[14,15]$. The $M$ line is situated at the median of the sarcomere, and it consists of several more structural proteins which anchor the thick filaments [16]. A key structural protein which maintains the integrity of the sarcomere is titin $[17,18]$. The precise distribution of the thin and thick filaments aided by the structural proteins found in the Z-disc and M-line provides the architecture for muscle contraction [19].

Several thousand sarcomeres arranged from end to end form the myofibril [1]. These myofibrils could span the entirety of the muscle [1]. A myofibril is surrounded by a membranous compartment called the sarcoplasmic reticulum [1]. The sarcoplasmic reticulum converges together to form the terminal cisterns, and these membranous sacs store vast amounts of $\mathrm{Ca}^{2+}$, which are released into the myofiber cytoplasm (also called the sarcoplasm), to initiate muscle contraction [20]. The sarcoplasm also contains myonuclei and mitochondria [1]. The transverse tubules (T-tubules) are invaginations of the plasma membrane of the myofiber, also called the sarcolemma, and these tubes bisect two terminal cisterns to form a triad [20]. The T-tubules in the myofiber serves to couple the muscle action potential to the contraction of the sarcomere [21]. 


\subsection{Skeletal muscle function}

The precise arrangement and structure of the skeletal muscle system allows for a range of movement such as walking and running, to finer subconscious actions such as postural adjustment while sitting or standing [3]. Muscle contraction generates heat, and as such, the rapid involuntary contractions of the muscle in response to the cold can drastically increase the body temperature [1]. Additionally, the involuntary contractions of the skeletal muscle system aid the circulation of lymph to the lymph nodes and blood to the heart [22]. It is only recently that the skeletal muscle is being appreciated as an endocrine organ that not only regulates the metabolism of itself, but also other tissues such as the adipose depots, liver, and even the brain $[2,23]$. On top of being a storage site for numerous metabolites like glucose and fatty acids, the skeletal muscle also secretes several muscle specific hormones, termed myokines, which can regulate the metabolism of the whole body [24].

\subsection{Skeletal muscle fiber types}

The functional diversity of the skeletal muscle can be attributed to several properties. The skeletal muscle is responsive to electrical signals generated from neurons [3]. They can either contract or extend without accruing significant damage, and they are highly elastic, often returning to its original shape and structure [1]. The skeletal muscle is a highly plastic tissue, as it is capable of regenerating and extreme remodeling to better suit certain cellular demands and stresses [25, 26]. Despite appearing relatively homogenous, the skeletal muscle fibers demonstrate remarkable differences in their gene expression, mitochondrial density, contraction time, and ATPase activity [3]. Muscle fibers are typically categorized into different groups depending on the myosin heavy chain (MHC) isoform expression [27, 28]. Slow twitch muscle fibers express MHC type I [27, 28]. They are rich in mitochondria, demonstrate increased vasculature, use oxidative phosphorylation as their main source of energy, and are resistant to fatigue $[27,28]$. On the other hand, muscle fibers which express type MHC IIb and IIx are fast twitch fibers $[27,28]$. These are functionally opposite to the slow twitch fibers in that they exhibit much greater contraction force but fatigue easily [27, 28]. Moreover, these fibers are limiting in the number of mitochondria, and consequently use anaerobic metabolism to generate ATP $[27,28]$. The fibers which express MHC type IIa share the qualities of both type I and type IIb/x fibers [27, 28]. 


\subsection{Skeletal muscle atrophy}

The skeletal muscle is an malleable tissue that can remodel itself in response to numerous types of stimuli [1]. While weight training and resistance exercise can induce the hypertrophy of the skeletal muscle, certain pathologies such as cancer or Duchenne's muscular dystrophy can promote the atrophy of the skeletal muscle $[5,29]$. Skeletal muscle atrophy, also termed as cachexia, is characterized by the reduction in the myofiber size and muscle mass [30]. The decrease in fiber size and muscle volume are also associated with a drastic change in the muscle fiber type profile $[31,32]$. The phenotypic changes that occur in the skeletal muscle during atrophy are now receiving intense scrutiny as cachexia is a salient feature of several other pathologies such as type 2 diabetes, AIDS, and sepsis [33-35]. The following section will focus on the mechanisms of skeletal muscle loss observed during cancer, termed cancer cachexia.

\subsection{Cancer cachexia}

Cancer cachexia is defined as the loss of the skeletal muscle mass due to the tumor burden [36]. However, patients inflicted with cancer cachexia exhibit a highly variable loss of adipose tissue [36]. Cancer cachexia was traditionally associated as a terminal condition in patients with advanced or untreatable malignancies [36]. However, the loss of body weight and skeletal muscle mass can be an early symptom of cancer. For instance, a study of 103 men who developed cancer in a group of 3,805 type IIa hyperlipidemic patients demonstrated a $1.2 \mathrm{~kg}$ reduction in body weight several months before diagnosis [37]. The prevalence of cancer cachexia is dependent on the type of cancer. In fact, over 50 to $85 \%$ of patients who suffer from gastric, pancreatic, colorectal, and lung malignancies demonstrated cancer cachexia at diagnosis and before the commencement of chemotherapy $[6,38]$. Other types of cancers such as non-Hodgkin lymphoma, and sarcomas manifest a lower incidence of weight loss [39].

Several studies have correlated the loss of the sarcomeric protein content, reduced myofiber area, and decreased muscle function due to cancer. In one study, the muscle biopsies of pancreatic cancer patients inflicted with symptoms of cachexia exhibited approximately $45 \%$ reduction in myosin heavy chain and $18 \%$ reduction in actin content compared to non-cachectic controls [40]. Similarly, patients suffering from colorectal cancer also lost myosin heavy chain content in the rectus muscle [41]. Reduced sarcomeric protein levels observed in cancer patients have also been associated with the reduction in the myofiber cross-sectional area [42, 
43]. In addition, patients suffering from advanced gastrointestinal and non-small cell lung cancers displayed lowered hand grip strength and quadricep strength [44].

More important, the extent of weight loss and the loss of skeletal muscle mass is prognostic of survival. A recent study of 242 patients with pancreatic adenocarcinoma demonstrated that patients who displayed cachexia had a median survival of 483 days, approximately half the number of days of survival (893 days) when compared to weight stable patients [45]. The onset of cachexia renders the conventional treatment of malignancies extremely ineffective and sometimes dangerous to the cachectic patient [46]. In fact, lung cancer patients who were cachectic displayed an adverse reaction to chemotherapy and were associated with a markedly reduced rate of survival [47]. In addition to the therapeutic challenges that occur due to cancer cachexia, this syndrome has unfavorable effects on the welfare of the patient, such as impaired physical activity, reduced concentration, and mental alertness [48-50].

To treat cancer cachexia, an in depth understanding of the mechanisms which underpin the loss of the skeletal muscle due to cancer should be examined. Over the past decade, several signaling mechanisms have been implicated in the pathogenesis of cancer cachexia. The following section will review the principal signaling events that occur during cancer-induced skeletal muscle atrophy. 


\subsection{Signaling events during cancer cachexia}

Skeletal muscle mass and function is maintained by the balance between anabolic and catabolic events [51]. These tightly regulated processes ensure the renewal of cellular components without a change in the overall muscle mass [52]. A bias towards catabolism resulting from a combination of increased protein degradation and reduced protein synthesis contributes to the skeletal muscle atrophy observed during cancer cachexia [53-55].

\section{Protein degradation - the ubiquitin proteasome pathway}

A hallmark of increased skeletal muscle degradation in not only cancer cachexia but also several other forms of skeletal muscle atrophy is the elevated expression and activity of the ubiquitin proteasome pathway (UPP) (Figure 1.7.1) [56]. The UPP targets proteins for degradation by first attaching several poly-ubiquitin moieties to the target protein [57]. The poly-ubiquitinated protein will be recognized by the $26 \mathrm{~S}$ proteasome, a large multisubunit protease, and subsequently degraded into smaller peptides [58]. The targeting of a particular protein for degradation is accomplished by 3 classes of enzymes: E1, E2, and E3 [57]. The E1, known as the ubiquitin activating enzyme, binds to a free ubiquitin molecule through a thiol ester intermediate catalyzed by the hydrolysis of ATP [57]. The E1 then transfers the ubiquitin to the E2, the ubiquitin conjugating enzyme, through another high-energy thiol intermediate [57]. Finally, the E3 ubiquitin ligase catalyzes the transfer of ubiquitin from the E2 and covalently attaches it to the substrate [57]. Upon ubiquitination, the target protein is destined to the proteasome for degradation [58]. 


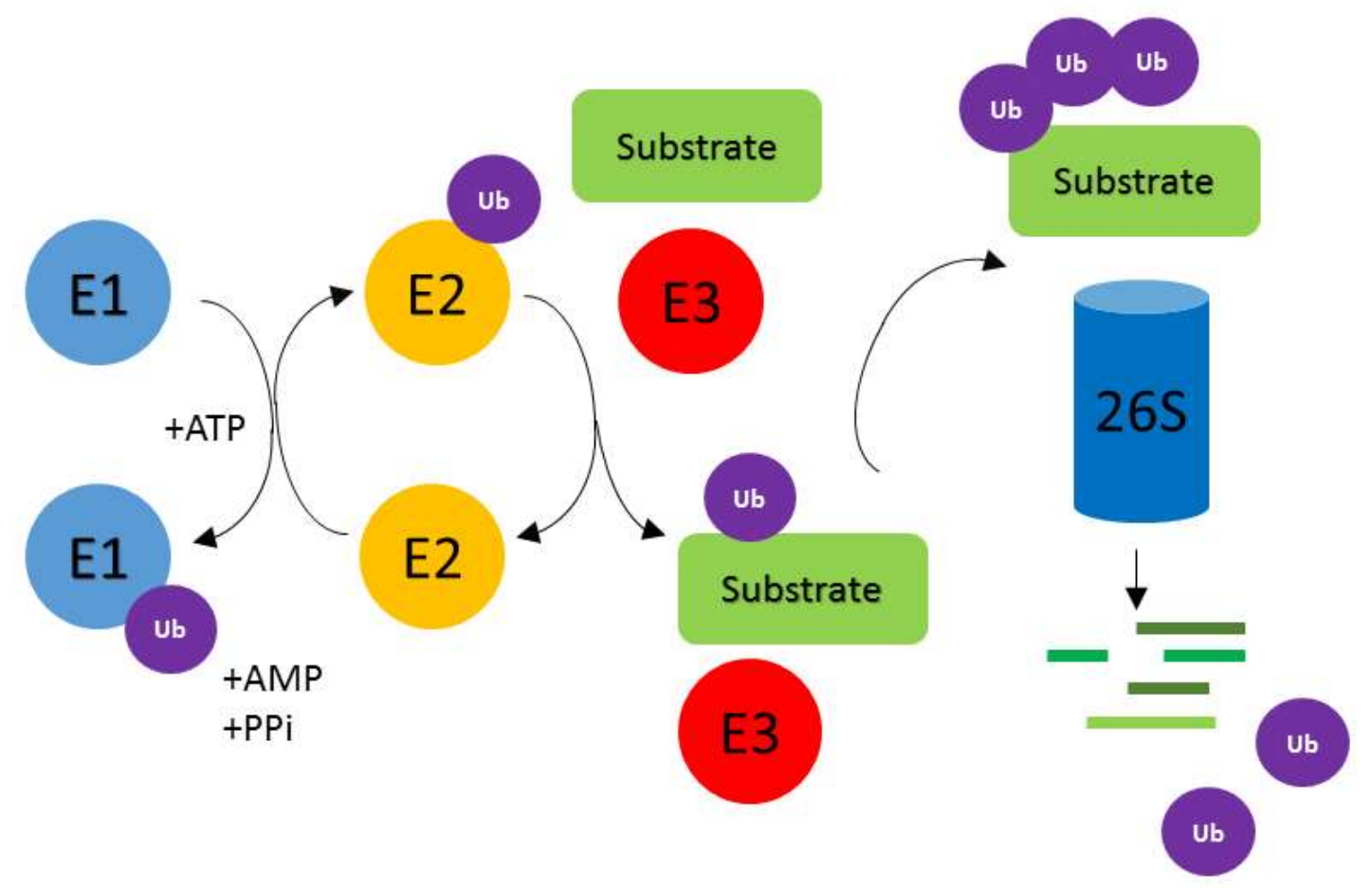

Figure 1.7.1: The ubiquitin proteasome pathway (UPP).

The E1 ubiquitin activating enzyme associates with free ubiquitin (Ub, purple) by hydrolyzing ATP. The E1 enzyme then transfers the ubiquitin molecule to the E2 ubiquitin conjugating enzyme. In a series of concerted steps, the E2 associates with the E3 ubiquitin ligase to transfer the ubiquitin molecule to the substrate. The substrate would be tagged by multiple ubiquitin molecules after several cascades of the UPP. These polyubiquitinated substrates are subsequently recognized and degraded by the $26 \mathrm{~S}$ proteasome, resulting in the release of smaller peptides and free ubiquitin molecules.

The elevated activity of the UPP in the skeletal muscles has been recapitulated in murine models of denervation and fasting, as well as mice inoculated with pro-inflammatory cytokines such as interleukin-6 (IL-6) and tumor necrosis factor alpha (TNF $\alpha$ ) [59-61]. The two key mediators of the UPP in skeletal muscle are muscle RING finger 1 (MuRF1, also known as TRIM63) and muscle atrophy $\mathrm{F}$ box (MAFbx/Atrogin-1/Fbxo32) [62]. As their names may indicate, these two proteins are muscle specific E3 ligases. Skeletal muscles isolated from murine cancer cachexia models exhibited elevated levels of both Atrogin-1 and MuRF1 [60, 63]. However, the expression status of these two E3 ligases in the muscles of patients suffering from cancer cachexia is controversial. A recent publication by Yuan and colleagues demonstrated that over $65 \%$ of patients with cancer cachexia exhibited increased mRNA levels of both Atrogin-1 and MuRF1 [64]. The increased activity of the UPP in human skeletal muscles was also reported to be correlated with increasing disease severity in gastric cancer 
patients [65]. However, a large study involving 51 cachectic patients inflicted with pancreatic, esophageal-gastric, intestinal, and common bile duct malignancies failed to display any changes in Atrogin-1 and MuRF1 expression levels when compared to the non-cachectic controls [66].

Despite this lack of consensus, considerable headway has been made in elucidating the functions of these two E3 ligases in muscle wasting. The central role of these E3 ligases in mediating cachexia became apparent when the muscles of the MuRF1 and Atrogin-1 null mice maintained their muscle mass despite experimentally induced atrophy [67, 68]. Furthermore, MuRF1 appears to selectively degrade structural proteins, such as myosin heavy chain, myosin light chain, and myosin-binding protein $C$ during muscle atrophy [59, 69]. The substrates of Atrogin-1 include proteins that regulate translation and myogenesis, such as elongation initiation factor 3 subunit 5 (eIF3-f), MyoD, and calcineurin [70-72].

Recently, several other novel E3 ligases have been implicated in the pathogenesis of cancerinduced skeletal muscle wasting. While MuRF1 promotes the destruction of myosin heavy chain filaments, the degradation of actin and other thin filaments is facilitated by TRIM32 [59, 73]. TRAF6 is another E3 ligase that is associated with muscle atrophy and cancer cachexia $[74,75]$. This particular E3 ligase is quite unique in that it catalyzes the conjugation of Lys63linked polyubiquitin moieties [59]. Unlike Lys43-linked targets, the Lys63-linked proteins are subjected to proteasome-independent degradation, and are specifically cleared through the autophagy-lysosomal pathway $[76,77]$.

Protein degradation - the autophagy-lysosome pathway

In addition to the UPP, the autophagy-lysosome pathway is now an emerging regulator of skeletal muscle mass and metabolism [78]. Autophagy is a term to describe the intracellular digestion system that delivers cytoplasmic components to the lysosome [79]. There are three forms of autophagy: macroautophagy, microautophagy, and chaperone-mediated autophagy [78]. The process of macroautophagy occurs in three discrete steps: initiation, nucleation, and expansion [80]. First, the unc-51-like kinase 1/mammalian autophagy-related gene 13 and 101 (ATG13 and ATG101)/focal adhesion kinase family interacting protein of $200 \mathrm{kDa}$ (ULK1/ATG13/FIP200) assemble on a portion of the endoplasmic reticulum (ER), called the omegasome $[80,81]$. It must be noted that autophagosomes are known to emanate from the 
plasma membrane, mitochondria, endosomes, ER-Golgi compartments and ER-mitochondria junctions [80, 82-84]. Next, the activated ULK1/Atg13/FIP200 complex targets a class 3 phosphatidylinositol-3 kinase (PI3K) (Beclin 1/vacuolar sorting protein 15 (VPS15)/VPS34/ATG14) which induces the production of phosphatidylinositol 3-phosphates (PIP3) [80]. Lastly, the Atg12-Atg5-Atg16 complex facilitates the conjugation of the phosphatidylethanolamine (PE) to the microtubule-associated protein 1 light chain 3 (LC3), resulting in a double membrane-bound vesicle that is capable of engulfing cytoplasmic proteins and organelles [80]. Microautophagy is the invagination of vesicles from the lysosome [85]. Chaperone-mediated autophagy, unlike macro- or microautophagy, does not require vesicles to transport the cargo to the lysosome for degradation [86]. Proteins that possess a KFERQlike pentapetide motif is specifically recognized by the heat shock cognate $70 \mathrm{kDa}$ protein (HSC70), which in turn translocates the cargo directly into the lysosome through the lysosomal-associated membrane protein 2A (LAMP2A) receptor [86].

In skeletal muscles, macroautophagy is essential for the clearance of damaged mitochondria and accumulated protein aggregates [78]. Indeed, the removal of such insults from the skeletal muscle is a hypothesized role for the elevated activity of the autophagy-lysosomal pathway observed during endurance exercise in mice and humans [87-89]. Furthermore, the increased activity of the autophagy-lysosome pathway has been reported in three models of murine cancer cachexia and in muscle biopsies of patients suffering from upper gastrointestinal, pancreatic, esophageal, and lung cancers [90-94]. The importance of this pathway in the maintenance of the skeletal muscle is further underscored by studies on skeletal muscle specific knockouts of Atg5 and Atg7. These reports demonstrated that the deletion of both Atg5, an E3 ubiquitin ligase essential for autophagosome formation, and Atg7, a regulator of Atg5, resulted in drastic muscle weakness and atrophy $[95,96]$. Certain myopathies in humans, such as Pompe and Danon disease, manifest atrophy of the skeletal muscle due to the impairment of lysosome function, further confirming the importance of this pathway as a critical regulator of muscle function $[97,98]$.

Given the protective role of autophagy, the increased activity of this pathway observed in cancer cachexia and other forms of skeletal muscle wasting could be viewed as a response to protect the muscle from excessive atrophy. However, the over-expression of autophagy, studied by the deletion of mechanistic target of rapamycin (mTOR), nuclear assembly factor1 (Naf-1), and regulatory-associated protein of mTOR (Raptor) ensued in drastic muscle 
atrophy [99-101]. Hence, unlike the UPP, the autophagy-lysosomal pathway is a proverbial double-edged sword - too much or too little amount of autophagy would result in skeletal muscle atrophy.

\section{Protein synthesis}

While the enhanced activity of the UPP and the autophagy-lysosomal pathway augments protein degradation, several reports have also highlighted that the rate of protein synthesis in muscles is adversely affected during cancer-induced skeletal muscle wasting [102, 103]. A critical pathway that is deregulated in cancer cachexia and other forms of atrophy is the PI3KAkt signaling axis. To understand the role of protein synthesis during cancer cachexia, the PI3K-Akt signaling cascade will be discussed below.

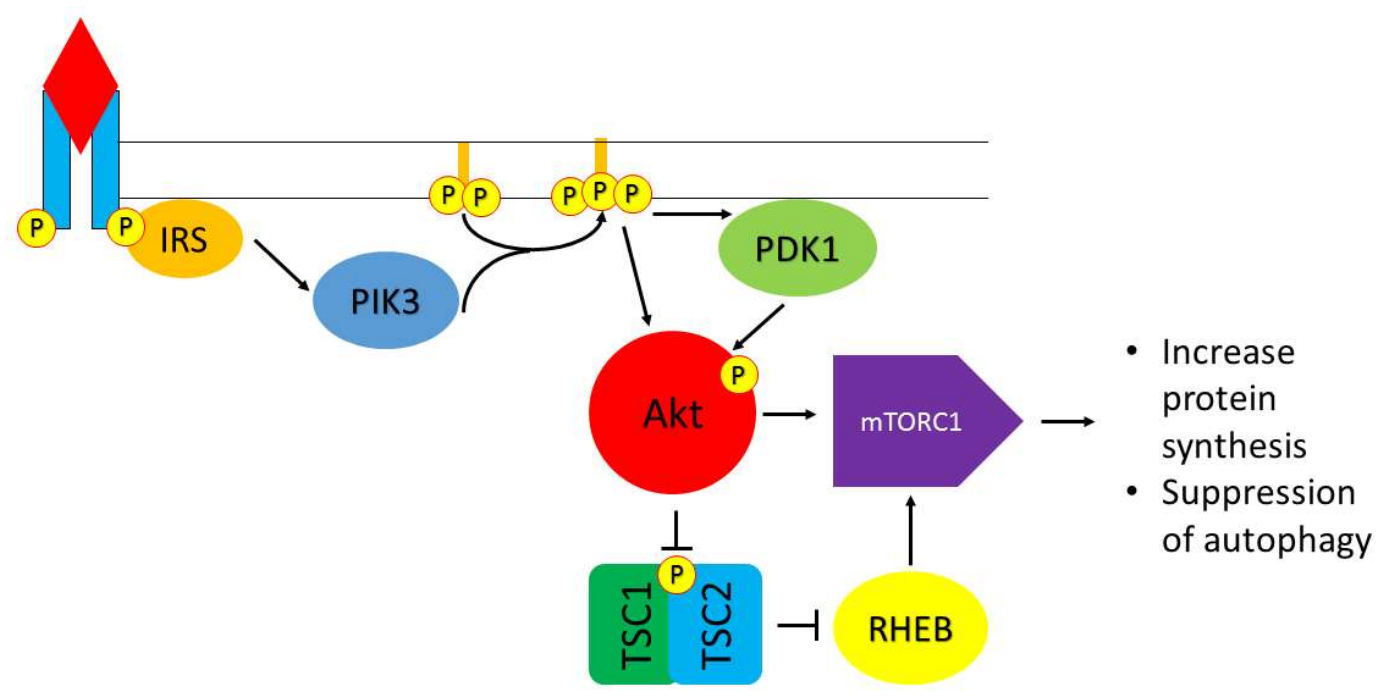

Figure 1.7.2: The PI3K-Akt pathway.

The binding of hypertrophic growth factors such as insulin-like growth factor 1 (IGF-1, red diamond) results in the activation and the autophosphorylation of the receptor tyrosine kinase (blue bars). The activated receptor then recruits the insulin receptor substrate (IRS), which is in turn phosphorylated by the intrinsic kinase activity of the receptor. The phosphorylated IRS acts as a docking site for phosphatidylinositol-3 kinase (PI3K), which catalyzes the phosphorylation of phosphoinositide-4,5-biphosphate (PIP2) to phosphoinositide-3,4,5-triphosphate (PIP3). PIP3 in turn will serve as a docking site for Akt and phosphoinositide-dependent kinase 1 (PDK1). PDK1 will phosphorylate Akt, resulting in its activation. Activated Akt can stimulate the mammalian target of rapamycin complex 1 (mTORC1) in two ways. First, activated Akt can phosphorylate and thus inactivate the tuberous sclerosis complex 1 and 2 (TSC1/2). TSC $1 / 2$ is a GTPase activating protein (GAP). When TSC1/2 is inactive, the Ras homolog enriched in brain (RHEB) would not be able to hydrolyze GTP to GDP. Thus, RHEB bound to GTP is a potent stimulator of mTORC1 activity. Second, Akt can directly stimulate the activity of mTORC1 by phosphorylating PRAS40, an inhibitor of mTORC1. Activated mTORC1 can thus perform numerous downstream functions such as increasing protein synthesis and suppressing autophagy, and thus promoting muscle hypertrophy. 
A simplified scheme of the PI3K-Akt pathway is shown above (Figure 1.7.2). In brief, the association of hypertrophic growth factors such as insulin-like growth factor 1 (IGF-1) to a receptor tyrosine kinase results in the stimulation of phosphatidylinositol-3 kinase (PI3K) [104]. PI3K phosphorylates phosphoinositide-4,5-biphosphate (PIP2) to phosphoinositide3,4,5-triphosphate (PIP3), where the latter serves as a potent docking site for Akt (also known as protein kinase B) and phosphoinositide-dependent kinase 1 (PDK1) [105]. PDK1 subsequently activates Akt by phosphorylating the serine 308 residue [105]. Akt elicits its anabolic effects in muscle by stimulating the activity of mTOR complex 1 (mTORC1), a large multisubunit complex comprising of mTOR and Raptor [99, 101, 105]. Specifically, activated Akt phosphorylates and thus inhibits a GTPase activating protein (GAP) called tuberous sclerosis complex 1 and 2 (TSC1/2) [106]. The inactivation of TSC1/2 results in the activation of Ras homolog enriched in brain (Rheb) [107]. In its GTP bound form, Rheb stimulates the kinase activity of mTORC1 [108]. TSC $1 / 2$ prevents the activation of mTORC1 by stimulating the GTPase activity of Rheb, thus rendering Rheb inactive [109]. Akt could also directly induce mTORC1 activation by phosphorylating the mTORC1 inhibitor, PRAS40 [110].

Active mTORC1 can regulate protein synthesis in three ways. Activated mTORC1 could promote translation by phosphorylating and inactivating the eukaryotic translation initiation factor 4E (eIF4E)-binding protein 1 (4E-BP1) [111]. The phosphorylation and subsequent activation of the S6 kinase 1 (S6K1) by mTORC1 results in the phosphorylation of several ribosomal proteins, which in turn would stimulate protein synthesis [112]. Finally, mTORC1 could also phosphorylate and inactivate glycogen synthase-3 beta (GSK3 $\beta$ ), which releases eukaryotic translation initiation factor 2B (eIF2B) from inhibition and thus promote translation [113]. Additionally, mTORC1 could also induce hypertrophy by suppressing autophagy [114]. Specifically, ULK1/Atg13/FIP200 is a direct target of mTORC1, which when phosphorylated results in impaired autophagy [115]. Given its central role in modulating hypertrophy in skeletal muscle, mTORC1 and its downstream components which regulate hypertrophy were found to be significantly repressed during skeletal muscle atrophy [102]. 
A master regulator of the skeletal muscle atrophy program is the forkhead box family, $\mathrm{O}$ subclass, (FoxO) transcription factor [116]. Skeletal muscle express three FoxO isoforms (FoxO1, FoxO3, and FoxO4) and these proteins aid in the transcription of the muscle E3 ligases, Atrogin-1 and MuRF1, and several components of the autophagy-lysosomal pathway, such as LC3B, Gabarap1, and Bnip3 [117, 118]. These genes which promote skeletal muscle wasting are collectively called atrogenes [119]. Constitutively active FoxO3 in skeletal muscle and skeletal muscle specific over-expression of FoxO1 were sufficient to induce acute myofiber atrophy, confirming the central role of the FoxO transcription factors as modulators of skeletal muscle wasting [116, 120]. All three isoforms of FoxO are regulated by Akt [121]. Indeed, increased Akt activity results in the phosphorylation and the subsequent accumulation of the FoxO transcription factors in the sarcoplasm [121, 122]. During muscle atrophy, the reduction in Akt activity results in the increased levels of the active, dephosphorylated form of FoxO, which in turn ensues in its nuclear translocation and the subsequent transcription of the atrogenes [123, 124].

\subsection{Extracellular signaling molecules involved in cancer cachexia}

The onset of cancer induced skeletal muscle wasting could be attributed to the accumulation of tumoral factors which facilitate the catabolism of the skeletal muscle, adipose tissue, and the liver $[125,126]$. The skeletal muscle, being a large repository for metabolites, can be viewed as an attractive target for the tumor to co-opt for its own survival [126].

There are several tumor derived factors which are postulated to induce the muscle wasting program observed during cancer. Tumor necrosis factor alpha $(\mathrm{TNF} \alpha)$, a pro-inflammatory cytokine secreted by neoplasms, is a potential mediator of cancer cachexia $[127,128]$. In vitro and in vivo experiments have demonstrated that the over-expression of TNF $\alpha$ promoted myofiber atrophy through the augmented activity of the UPP [129, 130]. While several rodent models have conclusively implicated $\mathrm{TNF} \alpha$ as a potent pro-cachectic factor, evidence confirming its importance in manifesting cancer cachexia in humans remains scant. In addition to studies reporting no difference in the TNF $\alpha$ expression in tumor-free and cachectic patients, antibodies against TNF $\alpha$ have also failed to ameliorate the muscle loss in patients with cancer cachexia $[131,132]$. 
Interleukin-6 (IL-6) is another tumor-derived cytokine that is implicated in the pathogenesis of cancer cachexia [133]. IL-6 has been associated as a pro-inflammatory cytokine that is noted in several instances of skeletal muscle atrophy both in mice and humans [134, 135]. Like TNF $\alpha$, IL-6 promotes the loss of skeletal muscle mass by stimulating the UPP [136, 137]. However, it is important to note that supraphysiological levels of IL-6 are required to elicit muscle wasting in healthy rodents [138]. These results may be due to the recent observations highlighting that IL-6 may also improve skeletal muscle glucose metabolism [139].

\subsection{Myostatin}

Myostatin (also called GDF-8), a transforming growth factor beta (TGF- $\beta$ ) superfamily member, is currently receiving intense scrutiny as a potential therapeutic target for the treatment of cancer cachexia and other muscle wasting disorders. It is synthesized predominantly in skeletal muscle, and, to a far lesser extent, in adipose tissue [140]. The importance of myostatin as a regulator of skeletal muscle biology was evident by the doublemuscle phenotype exhibited by animals such as the Belgian Blue cattle, Piedmontese cattle, and transgenic $\mathrm{Msth}^{-/}$mice [140-142]. Microscopic analysis of the skeletal muscles isolated from the $\mathrm{Mstn}^{-/-}$mice revealed that the double muscling phenotype was a result of both an increase in myofiber area (hypertrophy) and myofiber number (hyperplasia) [140]. In 2004, a case report identifying a boy who harbored a mutation in the myostatin locus demonstrated a similar hypermuscular phenotype, suggesting the highly conserved role of this growth factor in regulating skeletal muscle mass [143]. While the genetic ablation of myostatin resulted in muscle hypertrophy, systemic administration of this growth factor into mice precipitated the profound atrophy of the skeletal muscle [144]. Furthermore, increased expression of myostatin was detected in patients suffering from muscular dystrophy, after prolonged bed rest, and cancer cachexia [145-149].

\section{The processing and structure of myostatin}

Myostatin is translated as a 376 amino acid precursor protein that contains an $\mathrm{N}$-terminal signal sequence, a pro-peptide domain, and a C-terminal domain that encodes for the active growth factor (Figure 1.9.1) [150]. Myostatin undergoes two major proteolytic events: the N-terminal signal sequence, a 24 amino acid peptide that predestines this protein to secretory pathway, and the Arg-Ser-Arg-Arg (RSRR) site, located amino acids 266-267 from the start of the N-terminal 
signal sequence $[150,151]$. The cleavage of the RSRR site to separate the active myostatin from the $\mathrm{N}$-terminal pro-peptide is carried out by the pair dibasic amino-acid cleaving enzymes (PACE, also called furin) [152]. The furin enzymes, however, are not the only molecules that can assist in the maturation of myostatin. Indeed, in vitro experiments with muscle cell cultures in the presence of metalloprotease inhibitors resulted in the accumulation of unprocessed myostatin, suggesting the importance of these classes of proteases in regulating the maturation of myostatin [153].

Despite the separation of the pro-peptide from the mature C-terminal region after the cleavage reaction performed by furin, myostatin still remains non-covalently locked in an inactive latent state to the pro-peptide [144, 150, 154]. Administration of recombinant myostatin pro-peptide into rodents resulted in a striking increase in the muscle size and function, confirming the inhibitory effect of the pro-peptide on the function of myostatin [155-157]. In addition to its regulatory role, the pro-peptide also serves to modulate the folding of the signaling molecule $[158,159]$. One mechanism to render the active form of myostatin is another proteolytic cleavage event [160]. Specifically, Wolfmann and colleagues demonstrated that the aspartate 76 located on the pro-peptide is a site for proteolysis by the BMP1/Tolloid matrix metalloproteinases [160]. Mice harboring a point-mutation at the BMP1-mediated cleavage site yielded an increase in muscle mass as a result of increased myofiber number and size, despite the increased levels of myostatin circulation [161]. This improvement in muscle mass was significantly lower than the $\mathrm{Mstn}^{-/}$mice, indicating that there could be either some residual activity present in the myostatin complexed with the pro-peptide, or there could be other mechanisms that are able to liberate the pro-peptide from the active region.

Recent structural analysis of the biologically active C-terminal fragment reveals that myostatin may exist as a dimer (approximately $25 \mathrm{kDa}$ ) [162]. Each myostatin monomer appears to be oriented in an anti-parallel direction bound together by an intramolecular disulfide bond cysteine knot motif [162]. Although the amino acid sequence and the structure of the mature myostatin appear to be similar to several proteins in the TGF- $\beta$ family, particularly Activin A and GDF-11, myostatin appears to possess critical structural differences in the N-terminal region and the prehelix loop [162]. These differences have been postulated to govern the specificity of the myostatin dimer to its cognate receptors, activin type II B (ACVR2, also called ActRIIB), activin type 1B (ALK4), and TGF- $\beta$ receptor type 1 (ALK5) $[162,163]$. 


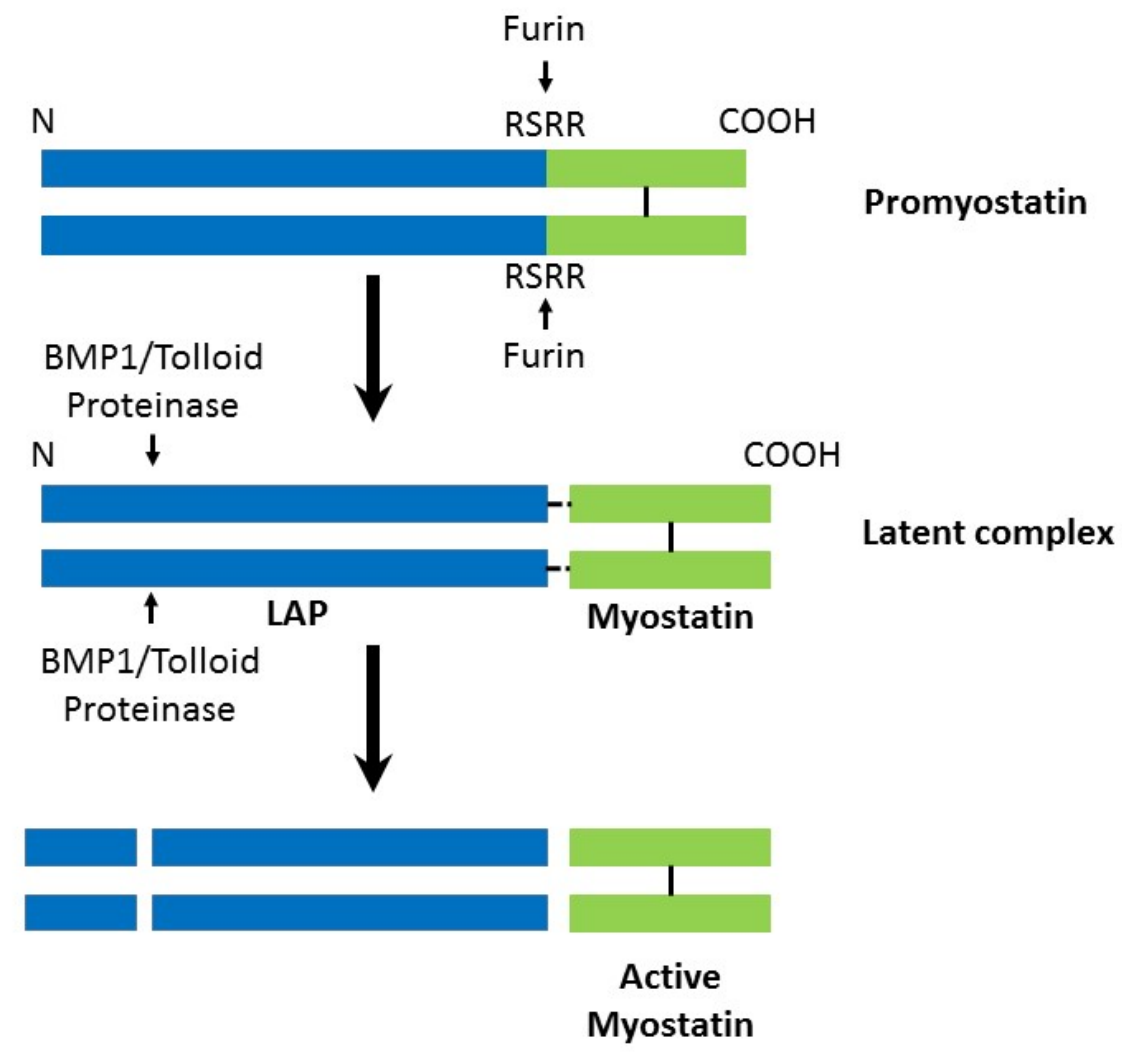

Figure 1.9.1: Processing of myostatin.

The maturation of myostatin commences with the cleavage of the RSRR site by the furin family of pro-protein convertases. This cleavage yields the latent associated peptide (LAP, blue) and the myostatin homodimer (green). The C-terminal myostatin peptide exists as a disulfide linked homodimer (black line). The LAP is bound non-covalently (black dotted line) to myostatin. The BMP1/Tolloid proteinase accomplishes the final cleavage event on the LAP, which results in the release of the biologically active myostatin from the latent complex.

The myostatin signaling pathway

Like most TGF- $\beta$ signaling proteins, myostatin binds to type I and type II TGF- $\beta$ serine/threonine kinase receptors to transduce its biological activity [164]. Myostatin binds to ActRIIB, and to a lesser extent, activin type $2 \mathrm{~A}$ receptors (ActRIIA), which in turn recruits the ALK4 and ALK5 type I receptors $[152,163]$. The binding of myostatin to its cognate receptors results in the formation of a heterotetrameric complex, followed by the activation of the ALK4/5 kinase $[152,163]$. The activated type I receptor recruits and subsequently phosphorylates the receptor Smads (R-Smads), which in the case for myostatin are Smad2 and Smad3 [165]. Next, the activated heterodimeric Smad2/3 associates with Smad4, a common partner Smad (Co-Smad), and translocate into the myonuclei to modulate the transcription of genes containing Smad binding elements (SBE) [165]. The signaling through the ActRIIB receptor can be attenuated by the inhibitory Smads (Smad 6 and 7), as these Smad proteins 
bind to ALK4/5 receptors and inhibit further signal transduction [166-168]. The myostatin signaling pathway also exhibits non-canonical routes of signal transduction. There is now accumulating evidence for the activation of the mitogen-activated protein kinase (MAPK) pathway and the suppression of the PI3K-Akt pathway by myostatin [169-172]. The details on how myostatin signals through these various signaling cascades to modulate the size of the skeletal muscle will be addressed below (Figure 1.9.2).

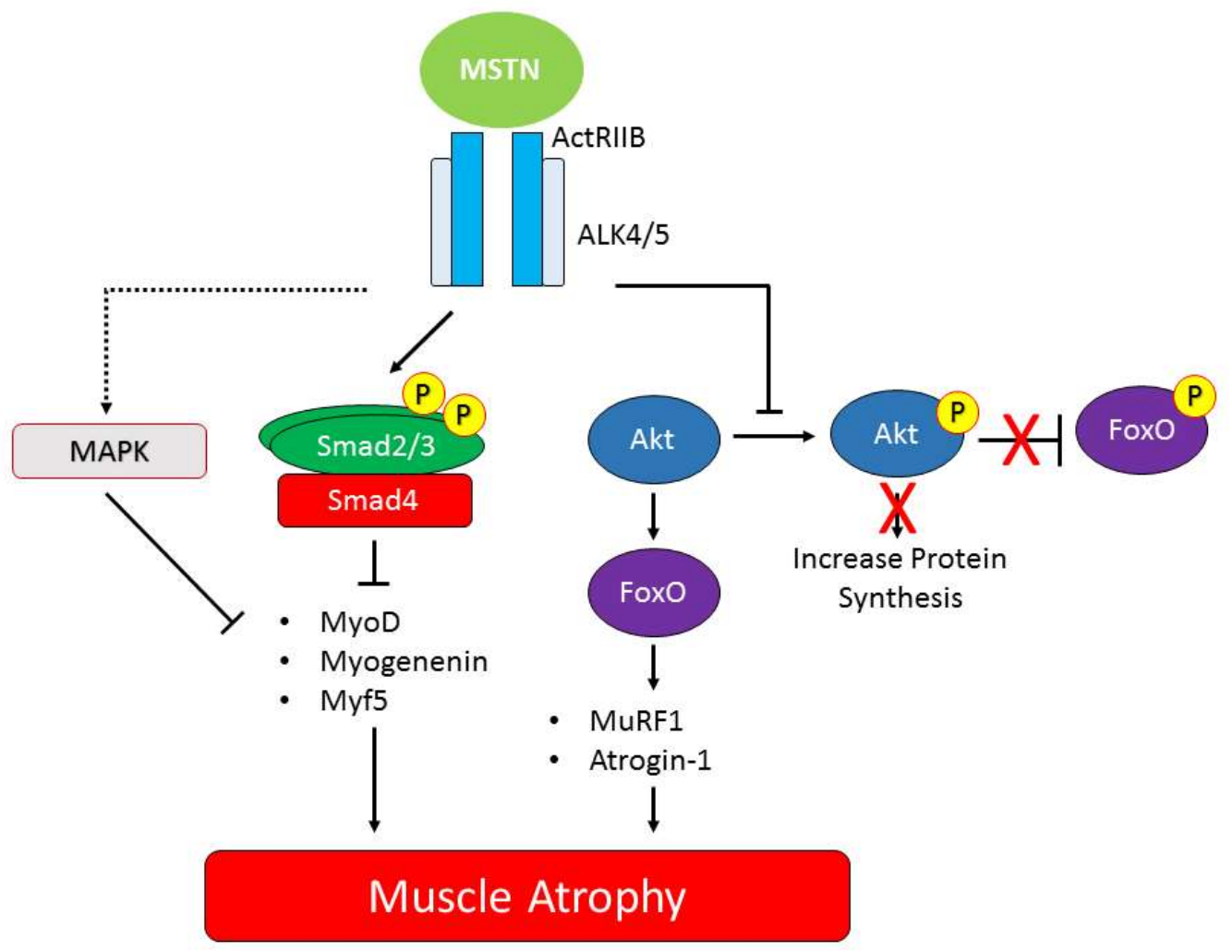

Figure 1.9.2: Myostatin signaling pathway.

The association of the myostatin to the ActRIIB receptor (blue bar) on the muscle membrane results in the recruitment of the ALK4/5 activin type 1 receptors (light blue bar). The activation of the ALK4/5 receptors results in the phosphorylation of Smad2 and Smad3. The Smad2/3 complex recruits Smad4, which translocates into the nucleus to inhibit the transcription of several myogenic genes. Myostatin also increases the activity of the MAPK pathway (p38), which also suppresses the genes involved in myogenesis. The dotted line connecting myostatin/ActRIIB/ALK4/5 complex represents the existence of intermediate steps that are currently unknown. Myostatin binding to the receptor also inhibits PI3K-Akt-mTOR pathway. Reduced Akt activity suppresses FoxO phosphorylation (highlighted by the red $\mathrm{X}$ ). Dephosphorylated FoxO can translocate into the nucleus and transcribe atrogenes such as MuRF1, Atrogin-1, and the autophagy-lysosomal pathway. The inhibition of PI3K-Akt-mTOR pathway by myostatin reduces protein synthesis. Collectively, the concerted induction and attenuation of key pathways by myostatin results in skeletal muscle atrophy. 
Given that myostatin knockout mice demonstrated increased myofiber hypertrophy and hyperplasia, it is postulated that myostatin could regulate both embryonic and postnatal muscle development. Indeed, work from our laboratory and others have demonstrated that the overexpression of myostatin significantly reduces the proliferation of myoblasts by controlling the G1 to $\mathrm{S}$ phase transition through a retinoblastoma $(\mathrm{Rb})$-dependent mechanism [173-175]. Specifically, myostatin treatment and endogenous over-expression ensued in the increased expression of $\mathrm{p} 21$, resulting in the accumulation of the hypophosphorylated form of $\mathrm{Rb}$ [173175]. The suppression of myogenesis by myostatin was also found to also occur through the p38 MAPK pathway [169]. It was discovered that the myostatin dependent activation of the p38 MAPK pathway was promoted by the TGF- $\beta$ activated kinase (TAK1) MAPK kinase (MAPKK) pathway, which in turn resulted in the upregulation of p21 [169]. In addition to the inhibition of myoblast proliferation, myostatin has also been found to inhibit myoblast differentiation [176-178]. The expression of critical myogenic transcription factors such as MyoD, myogenin, and Myf-5, were found to be depressed upon myostatin treatment [176-178]. The suppression of such pro-myogenic genes by myostatin were due to the increased activity of the Smad transcription factors [176-178].

The postnatal regulation of myofiber size as observed in the $\mathrm{Mstn}^{-/}$mouse suggests that myostatin may also regulate satellite cell activity and number. Satellite cells are quiescent muscle cells that are located between the sarcolemma and the basement lamina $[4,25]$. Upon injury, the satellite cell re-enters the cell cycle and proliferate to give rise to myoblasts, which in turn fuse with existing myofibers or form nascent myofibers [179]. Evidence for the regulation of satellite cells by myostatin was supported by the observation that $\mathrm{Mstn}^{-/}$mice demonstrated both an increased activity and number of such muscle stem cells [180, 181]. In addition, satellite cells isolated from $\mathrm{Mstn}^{-/-}$mice exhibited a greater proliferation rate in vitro as compared to wild type mice $[180,182]$. While it is unequivocal that myostatin is a potent regulator of muscle hyperplasia, whether the increased satellite cell activation in the absence of myostatin contributes to postnatal hypertrophy in skeletal muscles is fraught with controversy. In fact, mice treated with either a myostatin inhibitor or a dominant negative ActRIIB construct was sufficient to induce hypertrophy despite the absence of satellite cell activity and fusion $[183,184]$. Furthermore, elegant experiments conducted by Se-Jin Lee and 
colleagues demonstrated that the hypertrophy due to myostatin inhibition still occurred despite the inactivation of satellite cell in mice nullizygous for Pax7 and $S d c 4$ [185].

Several reports have highlighted that myostatin can regulate the size of the skeletal muscle by directly modulating the activity of the PI3K-Akt-mTOR pathway [186]. Reports have demonstrated that $\mathrm{Mstn}^{-/}$skeletal muscles exhibited an elevated expression and activity of several components of the PI3K-Akt-mTOR pathway $[187,188]$. Given the increased activity of PI3K-Akt-mTOR pathway in $\mathrm{Mstn}^{-/-}$skeletal muscle, it is unsurprising to note that myostatin also influences the translation capacity of the muscle. In particular, $\mathrm{Mstn}^{-/}$skeletal muscles exhibit increased myofibrillar protein synthesis, faster rate of translation factor assembly at the 7-methyl guanosine cap, and an increased RNA content [189-191]. Conversely, myostatin gene electrotransfer to the tibialis anterior of adult rats resulted in the suppression in PI3K-AktmTOR signaling [192]. Furthermore, the treatment of myostatin to in vitro muscle cell cultures was sufficient to attenuate the PI3K-Akt-mTOR pathway and reduce protein synthesis [172, 193].

In addition to suppressing skeletal muscle protein synthesis, myostatin can regulate skeletal muscle atrophy by stimulating the atrophy program in muscles. Signaling through the ActRIIB receptors upon myostatin interaction results in the activation of Smad2/3 and FoxO transcription factors $[163,171]$. As described prior, the activation of the FoxO transcription factors ensues in the upregulation of over 100 atrogenes, all of which impinge on the skeletal muscle to induce muscle wasting [62]. The gain- and loss-of-function studies on the Smad2, Smad3, and Smad4 proteins have also confirmed that these transcription factors activated by myostatin are potent inducers of skeletal muscle atrophy [184, 194]. Furthermore, myostatin treatment resulted in the increased levels of active FoxO1, which in turn promoted the expression of Atrogin-1 and several other genes involved in skeletal muscle wasting [171]. Myostatin expression itself can be upregulated by the increased activity of both the FoxO and Smad2/3 transcription factors [195]. Furthermore, $\mathrm{Mstn}^{-/}$skeletal muscles exhibit significantly lower amounts of Atrogin-1 transcript levels [196]. However, it must be noted that some studies have been unable to establish the link between myostatin and the expression of the atrogenes. Work by Trendelenburg and colleagues did not observe the upregulation of the atrogenes in human skeletal muscle cultures treated with myostatin in spite of the observed downregulation of Akt activity [172]. In addition, rat tibialis anterior ectopically over-expressing myostatin failed to exhibit the increased expression of the atrogenes [192]. 
Myostatin signaling has also been found to impinge on the exercise-induced transcription coactivator peroxisome proliferator-activated receptor- $\gamma$ coactivator-1 alpha (PGC1- $\alpha)$ in muscle. Myostatin inhibition was shown to increased PGC1- $\alpha$ activity in skeletal muscle, which in turn elevated mitochondrial biogenesis [197]. Furthermore, the elevated levels of PGC1- $\alpha$ is capable of suppressing the activity of the FoxO transcription factors [198]. More recently, a specific isoform of PGC1- $\alpha, \mathrm{PGC1}-\alpha 4$, was shown to increase the hypertrophy of murine skeletal muscles by down-regulating myostatin expression [199]. Moreover, the overexpression of PGC1- $\alpha 4$ was sufficient to prevent cancer-induced muscle wasting [199].

\subsection{Myostatin and cancer cachexia}

The first link between myostatin and cancer cachexia came from the siRNA mediated suppression of myostatin and the subsequent attenuation of skeletal muscle atrophy in a murine model of cancer cachexia [200]. Moreover, mice undergoing experimentally induced cancer cachexia treated with myostatin antagonists exhibited a remarkable increase in the body weight, improved skeletal muscle mass and function, and prolonged the life-span of the cachectic mice, in spite of the elevated levels of TNF $\alpha$ and IL-6 [63, 201, 202]. Despite the mounting evidence for a causative role in cancer-induced skeletal muscle atrophy, it remains to be seen whether a similar inhibition of myostatin by antibodies can prevent this metabolic syndrome in humans. Nonetheless, an initial phase 1 trial of advanced cancer patients treated with LY2495655, a myostatin antibody developed by Elli-Lily, reported an increase in muscle volume and hand grip strength [203]. Curiously, there appears to be no consensus on the expression status of myostatin in patients suffering from cancer [149, 204-207].

\subsection{Myostatin and cancer}

Studies on the effect of myostatin on malignancies are limited. Nevertheless, work by Langley et al. demonstrated that myostatin treatment inhibited the cellular proliferation of rhabdomyosarcoma (RMS) [208]. Conversely, the blockage of myostatin signaling by the expression of a dominant negative mutant of ActRIIB decreased RMS cellular proliferation through the MAPK signaling pathway [209]. Alternatively, a recent study has demonstrated that myostatin can suppress tumor growth in vivo, but the results of the report are objectionable due to the use of supraphysiological concentrations of myostatin [210]. To address this gap in knowledge concerning myostatin and cancer, we chose to specifically investigate how 
myostatin might regulate colorectal carcinogenesis. The next sections will address the mechanisms of colorectal cancer and intestinal biology. 


\subsection{Colorectal cancer}

Colorectal cancer is the penultimate cause of cancer mortality in the western world [211]. Furthermore, several studies have implicated colorectal cancers in manifesting cancer cachexia $[103,212-214]$. Colorectal cancers arise as a result of mutations that are acquired in a sequential manner [215]. Almost 80 to $90 \%$ of colorectal cancers are initiated by the loss of adenomatous polyposis coli (APC) [216]. The loss of this gene results in the accumulation of $\beta$-catenin, which in turn, through its association with TCF4/LEF1 transcription factors, results in the overexpression of numerous WNT target genes [216, 217]. Other mutations that are noted in colorectal cancers include KRAS, PTEN, TP53, and several components of the TGF- $\beta$ signaling pathway, such as SMAD2 and SMAD4 [218]. Colorectal tumors that do not harbor mutations in $A P C$ typically possess several mutations in the mismatch repair (MMR) genes, particularly MLH1 and MSH2 [219-221]. Unlike the mutations in APC which cause chromosomal instability, mutations in the MMR gene exhibit high levels of microsatellite instability and are often associated with improved prognosis [222-225]. To address the origin of colorectal cancers, the normal intestinal biology will be first introduced.

\subsection{The origin of colorectal cancers}

The intestine, particularly the intestinal epithelial cells (IEC), accomplishes numerous tasks, ranging from the digestion and absorption of food to maintaining a secure barrier against microorganisms and other carcinogenic insults [226]. While the majority of the intestinal epithelia are lined by absorptive enterocytes, there are several specialized secretory IECs which fulfill the multifaceted physiological roles of the intestine. Such secretory IECs include goblet cells, enteroendocrine cells, and Paneth cells [226]. The mucus secreting goblet cells and the Paneth cells, which produce antimicrobial proteins, assist in the establishment of a physical and biochemical barrier of the intestine [227, 228]. The 12 major enteroendocrine cell types that have been identified collectively synthesize and secrete approximately 20 peptide hormones [229]. The secretion of such hormones can elicit numerous responses from the intestine, ranging from modulating satiety, intestinal transit, digestive enzyme release, food absorption, immune response, and intestinal tissue growth [229].

IECs exhibit the highest rate of turnover [230]. It is estimated that approximately 200 grams of IECs (approximately $10^{11}$ cells) are lost every day in humans [231]. The replenishment of the 
intestinal epithelia is principally performed by the intestinal stem cell (ISC) [232]. A small number of these multipotent ISCs, located at the base of the intestinal crypt, divide to yield highly proliferative transit amplifying (TA) cells [233]. Over time, these TA cells migrate to the top of the villus and commit to either the absorptive or secretory linages [234, 235]. Paneth cells, on the other hand, arise from the base of the TA cell compartment, continue downwards towards the crypt [236].

The discovery of numerous molecular markers of the ISCs have bolstered the stem cell origin of colorectal cancer. A particular type of ISCs, called crypt base columnar cells (CBCs) are marked by the WNT target gene, Lgr5, a receptor for the Wnt agonist R-spondin [237]. Lineage tracing experiments have demonstrated that these cells reside at the base of the crypt and are capable of repopulating the entire intestinal epithelium [237]. In addition to $\operatorname{Lgr}^{+}$stem cells, another population of cells located 4 cells above the crypt base, also exhibit regenerative potential [238-240]. These label-retaining cells (LRCs), termed because they underwent continuous proliferation while retaining ${ }^{3} \mathrm{H}$-labelled DNA, are marked by Bmil, a component of chromatin silencing [241, 242]. These LRCs proliferate far less than $\operatorname{Lgr} 5^{+} \mathrm{CBCs}$, and more likely to function as a reserve cell in response to intestinal insults [243].

Numerous studies have demonstrated that the deletion of $\mathrm{Apc}$ in $\mathrm{Lgr}^{+}$and $\mathrm{Bmil}^{+}$stem cells, and other stem cell markers, ensue in the rapid formation of intestinal adenomas [241, 244247]. The hyperactivation of WNT signaling in the ISCs as the provenance for colorectal cancer is referred to as the bottom-up model [248]. Despite the overwhelming support for the crypt origin of colorectal cancers, there are instances where colorectal neoplasms have arisen from the non-stem cell compartment [249, 250]. Referred to as the top down model, this framework attempts to explain the formation of cancers at the top of the crypts, far removed from the stem cell rich crypts $[249,250]$. It is postulated that the increased activity of the NFKB pathway, through $K R A S$ activation or the administration of pro-inflammatory insults, ensues in the acquisition of pluripotency in otherwise differentiated IECs [251].

\subsection{Models to study colorectal cancer and cancer cachexia}

A suitable rodent model is required to investigate the role of myostatin in modulating both cancer cachexia and colorectal tumorigenesis. The $A p c^{\operatorname{Min} /+}$ mouse has been widely used to study the progression of cancer in the intestine [252]. Recently, the $A p c^{\mathrm{Min} /+}$ mouse is gaining 
attention as a model of cancer cachexia [253]. The $A p c^{M i n /+}$ mouse, generated by $N$-ethyl-Nnitrosourea (ENU) mutagenesis, possess a nonsense mutation at the codon 850 in one copy of the adenomatous polyposis coli (APC) gene [254-256]. The loss of heterozygosity (LOH) in the $A p c$ allele as the mouse ages results in the formation of numerous adenomas along the small intestine, and to a lesser extent in the colon [257, 258]. Female $A p c^{\mathrm{Min} /+}$ mice were also found to develop mammary carcinomas [254]. Mutations in the $A P C$ gene are accountable for the initiation and development of familial adenomatous polyposis (FAP) and several forms of sporadic colon cancers $[218,259]$. In addition, patients who are inflicted with colorectal carcinomas often exhibit cancer cachexia 6 months prior to diagnosis [260].

The $A p c^{\mathrm{Min} /+}$ mouse appears to be an attractive model to study cancer cachexia as it resembles the spontaneous occurrence of intestinal/colon cancer in humans [261]. It must be noted that the tumor burden in the $A p c^{\mathrm{Min} /+}$ mouse is strongly affected by its genetic background. $A p c^{\mathrm{Min} /+}$ mice on the C57BL/6 background develop approximately 30 intestinal tumors, but crossing these mice with other strains, such as AKR, MA, or CAST, profoundly reduces the tumor burden [262-264]. Through genetic linkage analysis, several groups have reported numerous genetic modifiers of the Min (MOM) phenotype, and these regions have been successfully correlated to their human functional equivalents [265]. Although it is important to note that $\mathrm{Apc}^{\mathrm{Min} / \mathrm{H}}$ mice should be studied on the C57BL/6 background, there have been instances of the spontaneous mutations that have resulted in a reduced tumor multiplicity within the same colony [266].

Another widely used model of intestinal tumorigenesis is the AOM/DSS model of colitisinduced colorectal carcinogenesis [267, 268]. Azoxymethane (AOM) is a tumor promoter that induces the formation of DNA-reactive adducts [269]. Specifically, the hydroxylation of AOM forms methylazoxymethanol, which is the principal compound that facilitates the alkylation of the $\mathrm{O}^{6}$ and $\mathrm{N}^{7}$ positions of guanine [270]. The induction of $\mathrm{O}^{6}$ methylguanine adducts in the DNA facilitate $\mathrm{G} \rightarrow \mathrm{A}$ mutations in the colon [271]. DSS is a heparin-like polysaccharide that damages the colonic epithelium and causes rapid inflammation, closely mimicking the symptoms of ulcerative colitis and irritable bowel disease (IBD) [272]. A single intraperitoneal injection of AOM, followed by one to three cycles of DSS over a period of 10 weeks can induce several colorectal tumors regardless of the genetic background of the mouse [273]. The molecular features of the AOM/DSS induced colorectal tumors mimic human colorectal cancers as well $[267,268]$. These tumors also exhibit a deregulation of the WNT signaling 
pathway, display mutations in $K R A S$, increased levels of prostaglandins and other inflammatory cytokines [274-278]. Interestingly, this model has yet to be used to study cancer cachexia. 


\section{Thesis Aims}

Myostatin is a secreted TGF- $\beta$ superfamily growth factor that regulates skeletal muscle mass and function. The over-expression of myostatin is a salient feature of cancer-induced skeletal muscle wasting. It is unclear whether myostatin over-expression during cancer cachexia is an early event or solely observed at the terminal stages of this metabolic disease. Importantly, evidence for the role of myostatin in regulating tumorigenesis especially during cancer cachexia is still nascent and perhaps controversial. The aims of this thesis are as follows:

1) To determine whether the $A p c^{\mathrm{Min} /+}$ mouse is a suitable model that recapitulates cancer cachexia

2) To establish whether myostatin expression is an early or a late marker of cancer cachexia in the $\mathrm{Apc}^{\mathrm{Min} /+}$ mouse model of intestinal and colonic cancer.

3) To investigate whether the inactivation of myostatin prevented cancer cachexia in the $A p c^{\mathrm{Min} /+}$ mouse

4) To determine the role of myostatin in the tumorigenesis of intestinal neoplasms using the $\mathrm{Apc}^{\mathrm{Min} /+}$ mouse and the AOM/DSS model of colitis-induced carcinogenesis 


\section{Materials and Methods}

\subsection{Animal Experiments}

Wild type mice (C57BL/6) were obtained from the Center for Animal Resources, National University of Singapore (NUS-CARE). Apc ${ }^{\mathrm{Min} /+}$ mice (C57BL/6) were purchased from Jackson Labs (Bar Harbor, ME, USA). Mstn ${ }^{+/}$mice (C57BL/6) were a gift from Professor Se-Jin Lee (Johns Hopkins University, Baltimore, MD, USA). Mice were maintained on standard chow diet and housed in groups at a constant temperature of $\left(20^{\circ} \mathrm{C}\right)$ under a 12:12 hour artificial light:dark cycle. Mice received ad libitum access to food and water. Animal experiments were performed in agreement with the regulations and guidelines of the Nanyang Technological University Institutional Animal Care and Use Committee (NTU-IACUC), Singapore.

\subsection{Genomic DNA isolation from mouse ear tissue}

Genomic DNA isolation from a small piece of mouse ear tissue was performed according to the protocol listed at the Jackson Laboratory (Website: http://jaxmice.jax.org/support/genotyping/tail phenol.html). The ear tissue was placed into 0.5 $\mathrm{ml}$ of digestion buffer in $1.7 \mathrm{ml}$ Eppendorf tube (Axygen Cat No: MCT-175-C) and incubated overnight at $55^{\circ} \mathrm{C}$ with constant agitation at $550 \mathrm{rpm}$ using the Thermomixer Comfort (Eppendorf). The composition of the digestion buffer was as follows: $50 \mathrm{mM}$ Tris $\mathrm{HCl} \mathrm{pH} 8.0$, $100 \mathrm{mM}$ EDTA pH 8.0, $100 \mathrm{mM} \mathrm{NaCl}, 1 \% \mathrm{SDS}, 0.5 \mathrm{mg} / \mathrm{ml}$ proteinase K (Promega Cat No: V302B), and autoclaved MilliQ water. The $10 \mathrm{mg} / \mathrm{ml}$ proteinase $\mathrm{K}$ stock solution was diluted in $50 \mathrm{mM}$ Tris $\mathrm{HCl} \mathrm{pH} 8.0,10 \mathrm{mM} \mathrm{CaCl}_{2}$, and autoclaved MilliQ water.

After overnight digestion, the tubes were spun briefly. $0.7 \mathrm{ml}$ of neutralized phenol/chloroform/isoamyl-alcohol (25:24:1) (Sigma Cat No: 77617) was added and the tubes were shaken vigorously for 15 minutes. The samples were spun at 13,200 rpm for 10 minutes at $4^{\circ} \mathrm{C}$ (Eppendorf 5415R - FA-45-24-11, 04874). Between 200 to $400 \mu 1$ of the upper aqueous phase was transferred to a fresh $1.7 \mathrm{ml}$ or $2.0 \mathrm{ml}$ Eppendorf tube (Axygen Cat No: MCT-200C). $1 \mathrm{ml}$ of room temperature 100\% ethanol (Merck Cat No: 1.00983.2500) was added. The samples were mixed several times until a white DNA precipitate was observed. The samples were spun at 13,200 rpm for 10 minutes at $4^{\circ} \mathrm{C}$. The supernatant was removed, and the DNA pellet was washed with $-20^{\circ} \mathrm{C} 70 \%$ ethanol, and centrifuged at maximum speed for 10 minutes at $4^{\circ} \mathrm{C}$. The prior step was repeated twice. In some instances, the DNA pellets were stored in 
$70 \%$ ethanol at $-20^{\circ} \mathrm{C}$. After the $70 \%$ ethanol supernatant was decanted, the samples were briefly spun at top speed once more, and the residual $70 \%$ ethanol was removed using a pipette. The DNA pellets were air-dried for approximately 10 to 15 minutes, or until the DNA pellet became translucent. There were some instances where the DNA pellet was extensively gelatinous, and as such, the tubes were incubated at $65^{\circ} \mathrm{C}$ using the Thermomixer Comfort to assist in the drying process. The DNA pellet was resuspended in $200 \mu \mathrm{l}$ of autoclaved MilliQ water, and incubated at $65^{\circ} \mathrm{C}$ for 15 minutes using the Thermomixer Comfort. The samples were drawn through a pipette to aid in the resuspension of the DNA. The DNA was quantified using the NanoDrop 1000 (Thermo Scientific, Program ID: nd-1000 V3.8). The genomic DNA was stored at $-20^{\circ} \mathrm{C}$.

\subsection{Genotyping $\mathrm{Apc}^{\mathrm{Min} /+}$ mice}

To detect for the $A p c^{M i n /+}$ allele, end point analysis protocol described by the Jackson Laboratory was used. Approximately $7.5 \mathrm{ng}$ of genomic DNA was added to the Kapa Probe Fast qPCR master mix (Kapa Biosystems Cat No: KK4702). One reaction of the Kapa Probe Fast qPCR master mix contained the following: 1X Kapa Probe Fast qPCR, $0.33 \mu \mathrm{M}$ Primer ID 13779, $0.33 \mu \mathrm{M}$ Primer ID 13780, $0.17 \mu \mathrm{M}$ Primer ID 13781, $0.17 \mu \mathrm{M}$ Primer ID 13782, diluted in autoclaved MilliQ water. $6 \mu \mathrm{l}$ of the sample were loaded in duplicate into the HardShell® Low-Profile Thin Wall 96 skirted PCR plates (Bio-Rad Cat No: HSP9601), and sealed with the Microseal ${ }^{\circledR}$ 'B' adhesive seals (Bio-Rad Cat No: MSB1001). The PCR plate was spun at $1000 \mathrm{XG}$ for 1 minute on the Sorval Legend RT (Rotor ID: Heraeus Cat No: 75006445 , PCR Plate Adaptor: Thermo Scientific Cat No: 75006449R, Rubber Adaptor: Thermo Scientific Cat No: EPDM 20056846). The PCR conditions were as follows: $95^{\circ} \mathrm{C}$ for 10 minutes, followed by 40 cycles of $95^{\circ} \mathrm{C}$ for 5 seconds and $60^{\circ} \mathrm{C}$ for 15 seconds, with a heated lid. The PCR was performed on the Bio-Rad CFX1000 with the 6-FAM and CAL Fluor Gold 540 channels selected. The 6-FAM channel would detect for $A p c^{M i n /+}$ allele. The CAL Fluor Gold 540 channel would detect for the wild type allele. All reactions were performed with a positive, negative, and a no-template (autoclaved MilliQ water) controls. Please note that CAL Fluor Gold 540 channel was chosen to detect JOE (NHS Ester) fluorophore. 


\subsection{Stratification of cancer cachexia in the $\mathrm{Apc}^{\mathrm{Min} /+}$ mice}

The progression of cancer cachexia in $\mathrm{Apc}^{\mathrm{Min} /+}$ male mice was determined by a modified strategy described by Baltglavis and colleagues [137, 279]. The progression of cancer cachexia in the male $\mathrm{Apc}^{\mathrm{Min} / \mathrm{+}}$ mouse was determined as a function of body weight loss rather than age. This was because male $A p c^{\mathrm{Min} /+}$ mice exhibited a heterogenous loss of body weight as it aged. 18 male $\mathrm{Apc}^{\mathrm{Min} /+}$ mice were weighed on a weekly basis from 8 weeks of age. The mice were stratified into 4 groups based on their percentage body weight loss. The percentage body weight loss was calculated by their live body weight prior to sacrifice divided their peak body weight. $\mathrm{Apc}^{\mathrm{Min} / \mathrm{H}}$ mice that displayed a $4.8 \%$ to $7.2 \%$ body weight loss were grouped as early cachexia $(n=5)$. Mice that displayed $9.8 \%$ to $14 \%$ body weight loss were included in the intermediate cachexia group $(n=4)$. Mice that exhibited a greater than $20 \%$ body weight loss were segregated into the severe cachexia group $(\mathrm{n}=6) . \mathrm{Apc}^{\mathrm{Min} / \mathrm{+}}$ mice that did not demonstrate a change in body weight between 14 to 18 weeks of age were included in the non-cachectic group $(n=7) .14$ week old wild type mice were included in this trial. Mice were euthanized by $\mathrm{CO}_{2}$ inhalation.

\subsection{Soluble ActRIIB (sActRIIB) purification}

Histidine-tagged soluble ActRIIB (sActIIB) fusion protein was expressed in bacteria using the pET-16b expression system [280]. Briefly, the cDNA of the extracellular domain of murine ActRIIB was amplified by PCR as a BamH1 fragment. The fragment was cloned into a pET$16 \mathrm{~b}$ expression vector (Novagen) and transformed into BL21 (Promega). IPTG induced recombinant protein was purified by Ni-NTA agarose (Qiagen) and dialysed with $50 \mathrm{mM}$ Tris$\mathrm{HCl} \mathrm{pH} 8.0,150 \mathrm{mM} \mathrm{NaCl}$. The purification of this protein was accomplished by Professor Ravi Kambadur and Nathiya Subramaniyam.

\subsection{SActRIIB treatment of $A p c^{\mathrm{Min} /+}$ mice}

Male $\mathrm{Apc}^{\mathrm{Min} /+}$ mice were used to assay whether the inhibition of myostatin ameliorated the symptoms of cancer cachexia. The mice were randomly divided into two groups that received $5.0 \mu \mathrm{g}$ per gram body weight of sActRIIB $(\mathrm{n}=12)$ or dialysis buffer $(\mathrm{n}=11)$. The composition of dialysis buffer was as follows: $50 \mathrm{mM}$ Tris- $\mathrm{HCl} \mathrm{pH}$ 8.0, $150 \mathrm{mM} \mathrm{NaCl}$. The mice received $200 \mu \mathrm{l}$ sActRIIB or dialysis buffer intraperitoneally. The trial commenced once the mice reached 16 weeks of age. The two groups received their respective treatments three times a week for one month (12 injections in total). To measure the food consumption, we first 
separated the treatment groups at the start of the inoculation. The dialysis buffer treated group was separated into three cages, where two cages housed four mice, and one cage contained three mice. The sActRIIB treatment group were separated into four cages, each containing three mice. Food consumption was first determined by measuring the amount of fresh feed added per cage at the start of the week. Next, the food remaining per cage at the end of one week was weighed. Calculating the difference between the food added at the start of the week and the remainder at the end of the week yielded the amount of food consumed per week for the cage in question. The amount of food consumed per mouse was computed by dividing the food consumed per cage by the number of mice in the cage. It must be disclosed that three mice perished due to the sActRIIB dosage (see Table 7.6.1). This prompted us to reduce the sActRIIB dosage to $2.5 \mu \mathrm{g}$ per gram body weight. This dosage was increased to $4.5 \mu \mathrm{g}$ per gram body weight (see Table 7.6.1). As a result of the loss of the three mice, we were unable to collect data for the food consumed for one cage in the sActRIIB treatment group.

Another trial was performed on 14 week old male $A p c^{\mathrm{Min} /+}$ mice to determine whether the administration of sActRIIB could delay the onset of cancer cachexia. Mice were randomly divided into two groups, the sActRIIB $(n=6)$ group, which received between $3.0 \mu \mathrm{g}$ per gram body weight of sActRIIB, and dialysis buffer group $(n=7)$. The mice received $200 \mu 1$ sActRIIB or dialysis buffer intraperitoneally. The trial commenced once the mice reached 14 weeks of age. The two groups received their respective treatments three times a week for one month (13 injections in total). The dosage of sActRIIB administered to the treatment group was increased to $4.5 \mu \mathrm{g}$ per gram body weight (see Table 7.7.6). It must be noted that one sActRIIB treated mouse perished during the course of the experiment.

\subsection{Grip strength measurement}

Prior to the termination of sActRIIB and dialysis buffer treated $\mathrm{Apc}^{\mathrm{Min} /+}$ mice, mouse forelimb grip strength was measured (Muromachi Kikai Cat No: MK-380M). Grip strength was measured three times, each with a 30 second interval of rest. 


\subsection{Generation of $\mathrm{Mstn}^{+/-}:: \mathrm{Apc}^{\mathrm{Min} /+}$ and $\mathrm{Mstn}^{-/}:: \mathrm{Apc}^{\mathrm{Min} /+}$ mice}

To generate $\mathrm{Mstn}^{+/} \because: A p c^{\mathrm{Min} /+}$ mice, male $\mathrm{Apc}^{\mathrm{Min} /+}$ mice were first bred with $\mathrm{Mstn}^{-/-}$females. The $\mathrm{Mstn}^{-/} \because: A p c^{\mathrm{Min} /+}$ mice were generated by crossing the $\mathrm{Mstn}^{+/-}: \because A p \mathrm{C}^{\mathrm{Min} /+}$ males to $\mathrm{Msth}^{-/-}$ females. The $M s t n^{-/}: \because A p c^{M i n /+}$ were subsequently maintained by crossing to $\mathrm{Mstn}^{-/-}$female mice. To detect for the presence of the Mstn allele, mice were genotyped according to an established protocol [281]. In brief, $250 \mathrm{ng}$ of genomic DNA isolated from the ear tissue of the mice were added into the PCR master mix containing the following: 1X Taq Buffer with $\left(\mathrm{NH}_{4}\right)_{2} \mathrm{SO}_{4}, 2 \mathrm{mM} \mathrm{MgCl} 2,0.2 \mathrm{mM}$ dNTP (Fermentas Cat No: R0192), $0.25 \mu \mathrm{M}$ of both forward and reverse primer, 5 units of Taq DNA polymerase (EP0405). $50 \mu$ of the sample were loaded into $0.2 \mathrm{ml}$ Thermo-strips (Thermo Scientific Cat No: AB-1182). The tubes were briefly spun in microcentrifuge affixed with a $0.2 \mathrm{ml}$ rotary adaptor. The PCR conditions are as follows: $95^{\circ} \mathrm{C}$ for 3 minutes, followed by 40 cycles of $95^{\circ} \mathrm{C}$ for 30 seconds, $60^{\circ} \mathrm{C}$ for 30 seconds, and $72^{\circ} \mathrm{C}$ for 1 minute, followed by a final extension at $72^{\circ} \mathrm{C}$ for 10 minutes. The samples were stored at $4^{\circ} \mathrm{C}$. The PCR was performed on the VWR Doppio with a heated lid (VWR Cat No: VWR1732-1201). Either 1X DNA loading dye solution or 1X Purple Gel Loading Dye (New England BioLabs Cat No: B7024S) was added to the PCR samples. The PCR products were resolved on a $2.0 \%$ agarose gel (Invitrogen Cat No: 15510-027) with $1 \mathrm{X}$ Tris-Acetate-EDTA (TAE) Buffer, pH 8.0 (1st Base Cat No: BUF-3000-50X1L). 1kB plus ladder (Invitrogen Cat No: 10787018) was used as a molecular weight marker. The bands were visualized with ethidium bromide (Bio-Rad Cat No: 161-0433). Image of the agarose gel was acquired by the GelDoc XR+ Imaging System (Bio-Rad)

However, during the course of our project, we opted to change the breeding scheme where $\mathrm{Mstn}^{+/-} \because \mathrm{Apc}^{\mathrm{Min/+}}$ male mice were mated with $\mathrm{Mstn}^{+/-}$female mice. Using this heterozygote breeding scheme, we were able to generate the following genotypes: $\mathrm{Mstn}^{+/+}: \because \mathrm{Apc}^{\mathrm{Min} /+}, \mathrm{Msth}^{+/-}$ $\because A p c^{M i n /+}$, and $M_{s t n}{ }^{-/} \because A A p c^{M i n /+}$, along with their respective controls, $\mathrm{Mstn}^{+/+}: \because A p c^{+/+}, \mathrm{Mstn}^{+/-}$ $\because A p c^{+/+}$, and $M s t n^{-/-}: A p c^{+/+}$. We chose an elegant genotyping protocol devised by Rachagani and colleagues to detect for the $\mathrm{Mstn}^{-/-}$allele in one single PCR reaction [282]. $50 \mu \mathrm{g}$ of mouse ear tissue genomic DNA was added to the Kapa Probe Fast qPCR master mix (Kapa Biosystems Cat No: KK4702). One reaction of the Kapa Probe Fast qPCR master mix contained the following: 1X Kapa Probe Fast qPCR, 0.25 $\mu \mathrm{M}$ Primer ID Mstn1-5, $0.25 \mu \mathrm{M}$ Primer ID Mstn2-5, 0.25 $\mu$ M Primer ID Mstn3-5, diluted in autoclaved MilliQ water. $20 \mu \mathrm{l}$ of the sample were loaded into $0.2 \mathrm{ml}$ Thermo-strips (Thermo Scientific Cat No: AB-1182). The 
tubes were briefly spun in a microcentrifuge affixed with a $0.2 \mathrm{ml}$ rotary adaptor. The PCR conditions were as follows: $92^{\circ} \mathrm{C}$ for 3 minutes, followed by 39 cycles of $92^{\circ} \mathrm{C}$ for 15 seconds, $65^{\circ} \mathrm{C}$ for 30 seconds, and $72^{\circ} \mathrm{C}$ for 30 seconds, followed by a final extension at $72^{\circ} \mathrm{C}$ for 10 minutes. The samples were stored at $4^{\circ} \mathrm{C}$. The PCR was performed on the VWR Doppio with a heated lid (VWR Cat No: VWR1732-1201). Either 1X DNA loading dye solution or 1X Purple Gel Loading Dye (New England BioLabs Cat No: B7024S) was added to the PCR samples. The PCR products were resolved on a $2.0 \%$ agarose gel (Invitrogen Cat No: 15510027) with 1X Tris-Acetate-EDTA (TAE) Buffer, pH 8.0 (1st Base Cat No: BUF-3000-50X1L). $1 \mathrm{kB}$ plus ladder (Invitrogen Cat No: 10787018) was used as a molecular weight marker. The bands were visualized with ethidium bromide (Bio-Rad Cat No: 161-0433). Image of the agarose gel was acquired by the GelDoc XR+ Imaging System (Bio-Rad). Wild type and the nullizygote allele migrated at 450 and 200 base pairs, respectively. 


\subsection{AOM/DSS induction of colorectal tumors}

The induction of colorectal cancers using the AOM/DSS model was performed according to a previously established protocol [273]. Male $\mathrm{Msth}^{+/}$and female $\mathrm{Msth}^{+/-}$mice were bred to generate $\mathrm{Mstn}^{+/+}$and $\mathrm{Mstn}^{-/-}$male mice. At 8 weeks of age, $\mathrm{Mstn}^{+/+}$and $\mathrm{Msth}^{-/-}$mice were injected intraperitoneally with $10 \mathrm{mg} / \mathrm{kg}$ body weight of AOM (Sigma A5486-100MG). The stock solution of AOM $(10 \mathrm{mg} / \mathrm{ml})$ was prepared by adding $10 \mathrm{ml}$ of sterile autoclaved MilliQ water into the ampule containing AOM. These stocks were stored at $-20^{\circ} \mathrm{C}$. The working solution of AOM used for the intraperitoneal injections was prepared by diluting the stock solution to $1.5 \mathrm{mg} / \mathrm{ml}$ in $0.9 \%$ isotonic saline. The isotonic saline was made in the laboratory and was filter sterilized using either $250 \mathrm{ml}$ (Corning Cat No: 431096) or $500 \mathrm{ml}$ (Thermo Scientific Cat No: 566-0020) PES membrane filter units. One week after the AOM injection, the mice were treated with 2.5\% DSS (MP Biologicals Cat No: 160110) dissolved in autoclaved MilliQ water. The amount of $2.5 \%$ DSS added and the amount consumed on day 3 of treatment was measured. At day 3 , fresh $2.5 \%$ DSS was added. The DSS was removed on day 5 , and the amount consumed was recorded. The bottle containing the $2.5 \%$ DSS solution was covered in aluminum foil. The second cycle of $2.5 \%$ DSS treatment started two weeks after the end of the first $2.5 \%$ DSS cycle. The third cycle of $2.5 \%$ DSS treatment commenced two weeks after the end of the second $2.5 \%$ DSS treatment. The body weights were measured daily during the DSS treatment and at least 1 week after the end of the cycle. During the DSS cycle, the two genotypes were housed in different cages to ascertain the about of DSS consumed. Mice were sacrificed between 9 to 11 weeks after the AOM treatment. 


\subsection{Muscle, adipose, and spleen collection}

The tibialis anterior (TA), gastrocnemius (GAS), quadriceps (QUAD), soleus (SOL), and the extensor digitorum longus (EDL) were weighed, snap frozen in liquid $\mathrm{N}_{2}$, and stored in $-80^{\circ} \mathrm{C}$ till further analysis. Epididymal (EPI), inguinal (ING), and retroperitoneal (RETRO) adipose tissue were handled similarly. Spleen weights were only recorded. Tibia lengths were recorded using a Vernier caliper.

\subsection{Intestine preparation for polyp counting}

For the preparation of the small and large intestine for polyp counting, intestine was first separated from the stomach. The colon was then extricated by breaking the pelvic bone. The sphincter muscles were carefully dissected from the colon. It was ensured that the intestine was free from the mesenteric adipose tissue. The small intestine and the large intestine were next separated from the cecum. The intestinal segments were flushed with either ice cold phosphate buffered saline (Oxoid Cat No: BR0014G) or 10\% neutral buffered formalin (NBF) (Sigma Cat No: HT501128-4L) using an 18G gavage needle (Solomon Scientific Cat No: FTP-18-38). The intestine and colon were cut longitudinally and were flattened on a filter paper. Another filter paper was placed and stapled on top the intestines. The tissues were fixed in $10 \%$ NBF at $4^{\circ} \mathrm{C}$ overnight, and stored in PBS at $4^{\circ} \mathrm{C}$ until further analysis.

For the assessment of the tumor number, the intestine and colon were briefly stained in $0.1 \%$ methylene blue (Sigma Cat No: M4159) diluted in PBS, destained in 70\% ethanol and further washed in PBS until the polyps became visible. The intestine was placed on an over-exposed X-ray film and transferred to a dissection microscope (Olympus Cat No: SZ61) with a fiber optic light source (Olympus LG-PS2). The microscope was affixed with an ocular micrometer (Olympus Cat No: 240CMIO/100) set at 2X magnification. Using two pairs of tweezers, the intestines were scored for their tumor number and diameter. 


\subsection{Organoid culture of $\mathrm{Mstn}^{+/+}:: \mathrm{Apc}^{\mathrm{Min} /+}, \mathrm{Mstn}^{+/-}:: \mathrm{Apc}^{\mathrm{Min} /+}$ and $\mathrm{Mstn}^{-/-}:: \mathrm{Apc}^{\mathrm{Min} /+} \mathrm{small}$ intestine adenomas}

The isolation of small intestine cancer organoids were performed according to a protocol described by Sato and colleagues [283]. Male $\mathrm{Mstn}^{+/+}: \because A p c^{\mathrm{Min} /+}, \mathrm{Mstn}^{+/-} \because: A p c^{\mathrm{Min} /+}$, and $\mathrm{Msth}^{-/-}$ $\because A p c^{M i n /+}$ mice, generated from the $\mathrm{Mstn}^{+/} \because \because A p c^{M i n /+} \times \mathrm{Mstn}^{+/-}$breeding scheme, were sacrificed by $\mathrm{CO}_{2}$ asphyxiation. The intestine was carefully extricated from the mouse and cleared of the mesenteric adipose tissue. The small intestine was flushed thoroughly with ice cold phosphate buffered saline (PBS) (Oxoid Cat No: BR0014G). The small intestine and colon were cut longitudinally, flattened on a filter paper, and immediately transferred into a falcon tube containing $40 \mathrm{ml}$ of ice cold PBS.

The polyps were isolated from small intestines using a dissection microscope (Olympus Cat No: SZ61) set at 2X magnification, with a fiber optic light source (Olympus LG-PS2). The polyps were extricated from each segment using a pair of forceps and a scalpel (Braun Cat No: BB511). Approximately 10 to 20 polyps were isolated and transferred to $15 \mathrm{ml}$ falcon tube containing ice cold chelation buffer. The composition of the chelation buffer was as follows: 2mM EDTA pH 8.0, 5.6 mM Na $2 \mathrm{HPO}_{4}, 8 \mathrm{mM} \mathrm{KH}_{2} \mathrm{PO}_{4}, 96.2 \mathrm{mM} \mathrm{NaCl}, 1.6 \mathrm{mM} \mathrm{KCl}, 43.4$ $\mathrm{mM}$ of sucrose, $54.9 \mathrm{mM}$ D-sorbitol, $0.5 \mathrm{mM}$ of DL- dithiothreitol, prepared in autoclaved MilliQ water and filter sterilized with a Sartorius MiniStart Highflow $0.20 \mu \mathrm{m}$ filter (Sartorius Cat No: 16532). After the polyps were incubated in ice cold chelation buffer for 30 to 60 minutes, the liquid was carefully decanted, and washed with ice cold PBS. The tumor fragments were allowed to settle, and the PBS was carefully decanted. The tumor fragments then received $5 \mathrm{ml}$ of digestion buffer warmed to $37^{\circ} \mathrm{C}$. The composition of the digestion buffer was the following: 75 units/ml type XI Collagenase (Sigma Cat No: C9407), $125 \mu \mathrm{g}$ type II Dispase (Gibco Cat No: 17105-041), 2.5\% HI-FBS (Gibco Cat No: 16140-71), $1 \mathrm{X}$ penicillin/streptomycin (Gibco Cat No: 15140-122), dissolved in high-glucose DMEM and filter sterilized with a Sartorius MiniStart Highflow $0.20 \mu \mathrm{m}$ filter (Sartorius Cat No: 16532). The tumor fragments were incubated for 1 hour at $37^{\circ} \mathrm{C}$ in a shaking incubator (Thermo Scientific Cat No: MaxQ4450) with constant agitation at $90 \mathrm{rpm}$. After the incubation step, the tumor fragments were aspirated several times, and allowed to settle to the bottom. The supernatant was carefully decanted into a fresh $15 \mathrm{ml}$ falcon tube. The supernatant containing the tumor cells were centrifuged at $500 \mathrm{XG}$ for 5 minutes at room temperature (Kubota Cat No: 2800, Rotor ID: RS-1004, Sigma $15 \mathrm{ml}$ falcon tube adaptor Cat No: 13152). The digestion 
buffer was decanted and the pellet was washed with room temperature PBS. This solution was centrifuged at $500 \mathrm{XG}$ for 5 minutes. After carefully removing the PBS, the tumor cells were resuspended in ice cold growth factor (GF) reduced Matrigel -no Phenol Red (Corning Cat No: 356231). $35 \mu 1$ of the Matrigel solution was pipetted to a 24 -well plate prewarmed to $37^{\circ} \mathrm{C}$. The Matrigel-organoid mixture was allowed to polymerize in a $37^{\circ} \mathrm{C}, 5 \% \mathrm{CO}_{2}$ incubator (Thermo Forma Model No: 371$)$. After polymerization, the Matrigel bubble was overlaid with $0.5 \mathrm{ml}$ of organoid culture media pre-warmed to $37^{\circ} \mathrm{C}$. The composition of the organoid culture media was as follows: $10 \mathrm{mM}$ HEPES (Gibco Cat No: 156030-080), 1X Glutamax (Gibco Cat No: 35050-061), 1X N2 Supplement (Invitrogen Cat No: 17502-048), 1X B27 supplement (+ vitamin A) (Invitrogen Cat No: 17504-044), 1 mM N-acetyl cysteine (Sigma Cat No: A91655G), 1 X penicillin/streptomycin (Gibco Cat No: 15140-122), $50 \mathrm{ng} / \mathrm{ml}$ epithelial growth factor (EGF) (Invitrogen Cat No: PMG8041), in Advanced DMEM:F12 (Invitrogen Cat No: 12634010), and filter sterilized with a Sartorius MiniStart Highflow $0.20 \mu \mathrm{m}$ filter (Sartorius Cat No: 16532). Organoid culture media was changed every 3 to 4 days. Organoids were imaged using the Nikon eclipse TS 100 live cell imager equipped with the Digital Sight DS Fi1c and the DSL3 interface (Olympus Light Microscope Cat No: CKX41). The setting used to image the organoids was as follows: $4 \mathrm{X}$ objective, with bright field (BF) selected, followed by the image mode set at Full with Exp mode set at Prog.

Organoids were passaged by first removing the growth media. The Matrigel bubble was mechanically disrupted using a P1000 pipette tip. PBS was added to the well and further aspirated several times to facilitate the dissociation of the organoids and the Matrigel. The dissociated organoids were centrifuged at $500 \mathrm{XG}$ for 5 minutes, and the supernatant was carefully removed. Excess Matrigel was also removed using a Pasteur pipette affixed to a vacuum pump. The organoid pellet was resuspended and propagated in the same manner described previously.

The organoids were cryopreserved in Recovery ${ }^{\mathrm{TM}}$ Cell Culture Freezing Medium (Invitrogen Cat No: 12648-010). In brief, the Matrigel bubble was disrupted as described before, centrifuged at $500 \mathrm{XG}$ for 5 minutes, and resuspended in Recovery ${ }^{\mathrm{TM}}$ Cell Culture Freezing Medium. After overnight cooling at $-80^{\circ} \mathrm{C}$ in a Mr. Frosty ${ }^{\mathrm{TM}}$ (Thermo Scientific) containing isopropanol, organoids were transferred to liquid $\mathrm{N}_{2}$ for extended storage. 


\subsection{Protein isolation of skeletal muscles}

Approximately 40 to $50 \mathrm{mg}$ of GAS muscle was lysed in $1 \mathrm{ml}$ of ice cold RIPA buffer. The RIPA buffer composition was as follows: $50 \mathrm{mM}$ Tris $\mathrm{HCl} \mathrm{pH}$ 7.5, $150 \mathrm{mM} \mathrm{NaCl}, 1.0 \mathrm{mM}$ EDTA pH 8.0, $1.0 \%$ NP-40, 0.1\% SDS, 0.5\% sodium deoxycholate, 1 mM PMSF, $25 \mathrm{mM}$ sodium fluoride, $1 \mathrm{mM}$ sodium orthovanadate, $1 \mathrm{X}$ cOmplete protease inhibitor cocktail (Roche Cat No: 11873 580001). One stainless steel bead (Qiagen Cat No: 69989) was placed into the $2 \mathrm{ml}$ Eppendorf tube containing the GAS muscles. The sample were homogenized at $30 \mathrm{~Hz}$ for 2 minutes, four times in total, with the TissueLyser II (Qiagen Cat No: 85300). Protein extracts were centrifuged at 12,000 rpm for 15 minutes at $4^{\circ} \mathrm{C}$ (Eppendorf 5415R - FA-45-24-11, 04874). The clarified lysate was stored at $-80^{\circ} \mathrm{C}$.

\subsection{Protein isolation from $\mathrm{Mstn}^{+/+}:: \mathrm{Apc}^{\mathrm{Min} /+}, \mathrm{Mstn}^{+/-}:: \mathrm{Apc}^{\mathrm{Min} /+}$ and $\mathrm{Mstn}^{-/-}:: \mathrm{Apc}^{\mathrm{Min} /+}$ organoids}

After the removal of the organoid culture media, ice cold Cell Recovery Solution (BD Cat No: 354253) was added into each 24-well. The Matrigel bubble was carefully dislodged and transferred to a pre-chilled $1.7 \mathrm{ml}$ Eppendorf tube. The Matrigel was allowed to depolymerize for 1 hour at $4^{\circ} \mathrm{C}$. The sample was centrifuged at $500 \mathrm{XG}$ for 5 minutes at $4^{\circ} \mathrm{C}$ (Eppendorf 5415R - FA-45-24-11, 04874). The solution was decanted, and the pellet was washed twice with ice cold PBS. In between the wash steps, the sample was centrifuged at $500 \mathrm{XG}$ for 5 minutes at $4^{\circ} \mathrm{C}$ to pellet the organoids. The organoids were lysed with RIPA buffer. To aid in the lysis, the sample was aspirated several times through a P200 pipette. Lysates were sonicated twice at amplitude of 40, 2 second pulse, followed a 5 second rest, with all steps performed on ice (Misonix Ultrasonic Disintegrator Sonicator S-4000 with Microtip). Protein extracts were centrifuged at 12,000 rpm for 15 minutes at $4^{\circ} \mathrm{C}$ (Eppendorf 5415R - FA-45-24-11, 04874). The clarified lysate was stored at $-80^{\circ} \mathrm{C}$. 


\subsection{Immunoblotting}

Protein concentration was estimated by the Bio-Rad Protein Assay Dye-Reagent (Bio-Rad Cat No: 500-0006). Quick StartTM Bovine Serum Albumin (BSA) standard (Bio-Rad Cat No: 5000206) was used for preparing the protein standards. The protein concentration was estimated in duplicates using the xMark Microplate Spectrophotometer (Bio Rad Cat No: 1681150) and Microplate Manager 6 Software (Bio Rad Cat No: 1689520). The 96 well plate was read at 595 nm.

For immunoblotting, 15-40 $\mu \mathrm{g}$ of protein were resolved on NuPAGE Novex 4-12\% gels. Depending on the number of samples, the following NuPAGE gels were used: 10-well (Invitrogen Cat No: NP0321BOX), 12-well (Invitrogen Cat No: NP0322BOX), 20-well (Invitrogen Cat No: WG1402BX10), 26 well (Life Technologies Cat No: WG1403BX10). 1X NuPAGE LDS Sample Buffer (Invitrogen Cat No: NP0007) with $\beta$-mercaptoethanol (Sigma Cat No: M3148) (at a ratio of 4:1) was added to the protein samples and boiled for 5 minutes on a heat block (Techne Heating Block DB-3D FDB03DD). The protein gel was run at 150V in $1 X$ NuPAGE MES SDS Running Buffer (Invitrogen Cat No: NP0002). SeeBlue Plus2 PreStained Protein Standard (Invitrogen Cat No: LC5925) was used as a molecular weight marker. 20- and 26-well gels were transferred onto a $0.2 \mu \mathrm{m}$ nitrocellulose membrane (Bio-Rad Cat No: 162-0112) using the Trans-Blot Cell (Bio-Rad Cat No: 1703939) at 150V for 1 to 2 hours. 10- and 12-well gels were transferred onto the same nitrocellulose membranes using the XCell II Blot Module (Novex Cat No: EI9051) at 30V for 1 hour 30 for 1 gel, and 2 hours for 2 gels. The transfer buffer composition is as follows: $25 \mathrm{mM}$ Tris, $192 \mathrm{mM}$ glycine (Bio-Rad Cat No: 161-0718), 20\% methanol (Merck Cat No: 1.06009.2500), diluted in autoclaved MilliQ water. The quality of the transfer was verified by Ponceau S staining (Sigma Cat No: P7170). The blot was destained with $1 \mathrm{X}$ TBST. The composition of $1 \mathrm{X}$ TBST was as follows: $50 \mathrm{mM}$ Tris $\mathrm{HCl} \mathrm{pH} \mathrm{7.4,} 150 \mathrm{mM} \mathrm{NaCl}, 0.1 \%$ Tween-20 (Promega Cat No: 45151). The immunoblots were blocked in 5\% skimmed milk (Fluka Cat No: 70166) in 1X TBST for 1 hour at room temperature. Either 5\% skimmed milk in 1 TBST or $5 \%$ bovine serum albumin (BSA) (Sigma Cat No: A7906) in 1X TBST was used as the antibody dilution medium. Details of the antibodies used in this manuscript and their respective dilutions and conditions are listed in Table 1. At each step, the blots were washed 3 times for 5 minutes with 1X TBST. Immunoreactivity was detected by Western Lightning Plus-ECL (PerkinElmer Cat No: NEL105001EA). Immunoblots were scanned using the GS-800 Calibrated Densitometer (Bio 
Rad Cat No: 1707983). Densitometry was evaluated by Quantity-One 1D-Analysis Software (Bio-Rad Cat No: 1709600). 


\subsection{Preparation of skeletal muscle for RNA extraction}

Approximately 50 to $80 \mathrm{mg}$ of GAS muscle was lysed in $1 \mathrm{ml}$ of TRIzol reagent (Invitrogen Cat No: 15596018). One stainless steel bead (Qiagen Cat No: 69989) was placed into the $2 \mathrm{ml}$ Eppendorf tube containing the GAS muscles. The sample were homogenized at $30 \mathrm{~Hz}$ for 2 minutes, two times in total, with the TissueLyser II (Qiagen Cat No: 85300). The lysate was centrifuged at 12,000 XG for 10 minutes at $4^{\circ} \mathrm{C}$ (Eppendorf 5415R - FA-45-24-11, 04874). The clarified lysate was stored at $-80^{\circ} \mathrm{C}$ until further use.

\subsection{Preparation of intestinal tissue and polyps for RNA extraction}

After the extrication of the intestine from the mouse, the intestinal segments were flushed with ice cold PBS (Oxoid Cat No: BR0014G) using an 18G gavage needle (Solomon Scientific Cat No: FTP-18-38). The small intestine and colon were cut longitudinally and were flattened on a filter paper. The small intestine was segmented into 4 equal lengths. Their directionality was maintained during this procedure. The intestines were then stored in falcon tubes containing over $12 \mathrm{ml}$ of RNAlater solution (Ambion Cat No: AM7021) and left overnight at $4^{\circ} \mathrm{C}$.

After overnight immersion of RNAlater, wild type intestinal tissues were dried on a kimwipe and cut into 2 to $4 \mathrm{~cm}$ long pieces. These fragments were stored in a $2 \mathrm{ml}$ Eppendorf tube $80^{\circ} \mathrm{C}$ until further use. Polyps were isolated from the RNAlater immersed intestines using a dissection microscope (Olympus Cat No: SZ61) set at 2X magnification, with a fiber optic light source (Olympus LG-PS2). The polyps were extricated from each segment using a pair of forceps and a scalpel (Braun Cat No: BB511). Approximately 5 to 10 polyps were isolated per segment and stored in a $2 \mathrm{ml}$ Eppendorf tube at $-80^{\circ} \mathrm{C}$ until further use. 2 to $4 \mathrm{~cm}$ long intestinal tissues that were clear of macrotumors were also stored in a $2 \mathrm{ml}$ Eppendorf tube at $-80^{\circ} \mathrm{C}$ until further use.

The small intestinal tissue and polyps were lysed in $1 \mathrm{ml}$ of TRIzol reagent (Invitrogen Cat No: 15596018). One stainless steel bead (Qiagen Cat No: 69989) was placed into the $2 \mathrm{ml}$ Eppendorf tube containing the intestinal tissue or polyps. The sample were homogenized at 30 $\mathrm{Hz}$ for 2 minutes, two times in total, with the TissueLyser II (Qiagen Cat No: 85300). The lysate was centrifuged at $12,000 \mathrm{XG}$ for 10 minutes at $4^{\circ} \mathrm{C}$ (Eppendorf 5415R - FA-45-24-11, 04874). The clarified lysate was stored at $-80^{\circ} \mathrm{C}$ until further use. 


\subsection{RNA isolation from $\mathrm{Mstn}^{+/+}:: \mathrm{Apc}^{\mathrm{Min} /+}, \mathrm{Mstn}^{+/-}:: \mathrm{Apc}^{\mathrm{Min} /+}$ and $\mathrm{Mstn}^{-/}:: \mathrm{Apc}^{\mathrm{Min} /+}$ organoids}

TRIzol was added to each well containing the Matrigel-organoid bubble, and homogenized by passing the solution through a P200 pipette tip. The samples were allowed to rest at room temperature for 10 minutes, and stored at $-80^{\circ} \mathrm{C}$ until further use.

\subsection{RNA Isolation}

$200 \mu \mathrm{l}$ of chloroform (Merck Cat No: 1.02445.2500) was added to the TRIzol lysate and was mixed vigorously for 15 seconds. The sample was left to stand for 3 minutes, and was centrifuged at 12,000 XG for 15 minutes at $4^{\circ} \mathrm{C}$ (Eppendorf 5415R - FA-45-24-11, 04874). Between 400 to $650 \mu \mathrm{l}$ of the upper aqueous phase was transferred to a $1.7 \mathrm{ml}$ Eppendorf tube containing $0.5 \mathrm{ml}$ of isopropanol (Merck Cat No: 1.09634.2500). The sample was mixed and was incubated at $-20^{\circ} \mathrm{C}$ for 30 minutes. For organoid samples, where the yield of RNA was expected to be low, $10 \mu \mathrm{g}$ of glycogen (Ambion Cat No: AM9510) was added to the mixture. The sample was then centrifuged at top speed for 10 minutes at $4^{\circ} \mathrm{C}$ (Eppendorf $5415 \mathrm{R}-\mathrm{FA}$ 45-24-11, 04874). The supernatant was removed, and the RNA pellet was washed with 75\% ethanol in DEPC-treated MilliQ water, and centrifuged at maximum speed for 10 minutes at $4^{\circ} \mathrm{C}$. After the $75 \%$ ethanol in DEPC water was decanted, the samples were briefly spun at top speed once more, and the residual liquid was removed using a pipette. The RNA pellets were air-dried for approximately 10 to 15 minutes, or until the RNA pellet became translucent. The RNA pellet was resuspended in $84 \mu \mathrm{l}$ of autoclaved DEPC-treated MilliQ water, and incubated between 50 to $55^{\circ} \mathrm{C}$ for 15 minutes using the Thermomixer Comfort. The samples were drawn through a pipette to aid in the resuspension of the RNA. The samples were chilled on ice for 5 minutes, then either stored at $-80^{\circ} \mathrm{C}$ until further use or treated with DNase.

For the DNase treatment of RNA, $16 \mu$ of the DNase master mix (Promega Cat No: M6101) was added to the resuspended RNA sample. The DNase master mix was composed of the following: 1X DNase Buffer, 5 units of RQ1 RNase-free DNase, 20 units of recombinant RNasin ${ }^{\circ}$ ribonuclease inhibitor (Promega Cat No: N2511). The sample was incubated at $37^{\circ} \mathrm{C}$ between 30 to 40 minutes on a Thermomixer Comfort. After the incubation, $300 \mu 1$ of DEPCtreated water was added, followed by the addition of $400 \mu \mathrm{l}$ of acid phenol:chloroform (Ambion Cat No: AM9720). Please note, that the stock acid phenol:chloroform was supplemented with 
additional chloroform ( $2 \mathrm{ml}$ of chloroform to $3 \mathrm{ml}$ of acid phenol:chloroform). The samples were mixed vigorously for 15 seconds, and allowed to sit for approximately 3 minutes. The mixture was centrifuged at top speed for 10 minutes at $4^{\circ} \mathrm{C} .350 \mu 1$ of the upper aqueous phase was transferred to a fresh $1.7 \mathrm{ml}$ Eppendorf tube containing $40 \mu \mathrm{l}$ of $3 \mathrm{M}$ sodium acetate $\mathrm{pH}$ 5.2 (Sigma Cat No: S7899) and $1 \mathrm{ml}$ of $100 \%$ ethanol. The sample was mixed and stored at $80^{\circ} \mathrm{C}$ for 1 hour. The sample was centrifuged at top speed for 10 minutes at $4^{\circ} \mathrm{C}$, and the supernatant was carefully decanted. The RNA pellet was washed with $75 \%$ ethanol in DEPCtreated MilliQ water, and centrifuged at maximum speed for 10 minutes at $4^{\circ} \mathrm{C}$. The RNA pellet was stored in $75 \%$ ethanol in DEPC-treated MilliQ water in $-20^{\circ} \mathrm{C}$ until further use. After centrifugation, the supernatant was removed and the sample was spun briefly at top speed, and the residual $75 \%$ ethanol in DEPC-treated MilliQ water was removed using a pipette. The RNA pellets were air-dried for approximately 10 to 15 minutes, or until the RNA pellet became translucent. Depending on the size of the pellet, the RNA was dissolved in 30 to $200 \mu$ of autoclaved DEPC-water and heated between 50 to $55^{\circ} \mathrm{C}$ for 15 minutes on the Thermomixer Comfort. The tubes were chilled on ice for 5 minutes. RNA was quantified using the NanoDrop 1000 (Thermo Scientific, Program ID: nd-1000 V3.8). RNA was stored at $-80^{\circ} \mathrm{C}$.

\subsection{RNA Integrity}

RNA integrity was assayed by denaturing RNA gel electrophoresis. In brief, between 0.5 to $1.0 \mu \mathrm{g}$ of RNA was mixed with 1X RNA loading dye and heated to $65^{\circ} \mathrm{C}$ for 10 to 15 minutes using the Thermomixer Comfort. The samples were cooled on ice for 2 minutes, and then loaded to an agarose gel containing 1.2\% agarose (1st Base Cat No: 1A0115M15447), 1X MOPS, 6.6\% formaldehyde (Sigma Cat No: Cat No: F8775). The bands were visualized with ethidium bromide (Bio-Rad Cat No: 161-0433). Image of the agarose gel was acquired by the GelDoc XR+ Imaging System (Bio-Rad).

\subsection{Real time quantitative qPCR (RT-pPCR)}

Either 0.5 or $1 \mu \mathrm{g}$ of RNA was used for the synthesis of cDNA (Bio Rad Cat No: 170-8891). The reaction was conducted according to the manufacturer. In brief, the RNA and the iScript cDNA Synthesis master mix was set up in 8-tube strips (Bio-Rad Cat No: TLS0801) and covered by the optical flat 8-cap strips (Bio-Rad Cat no: TCS0803). The cDNA synthesis protocol was performed on the Bio-Rad CFX1000 with the following conditions: 5 minutes at 
$25^{\circ} \mathrm{C}, 30$ minutes at $42^{\circ} \mathrm{C}$, and 5 minutes at $85^{\circ} \mathrm{C}$. The samples were held at $4^{\circ} \mathrm{C}$. After the cDNA synthesis, cDNA was diluted in autoclaved MilliQ water to 1:20 or 1:30 for skeletal muscles, 1:30 for intestinal tissues, and 1:10 for cancer organoids. Gene expression was studied by using the SsoFast EvaGreen Supermix (Bio-Rad Cat No: 7500001227). $3 \mu$ of cDNA was added to $7 \mu \mathrm{l}$ SsoFast EvaGreen Supermix per well. The SsoFast EvaGreen Supermix master mix consisted of the following: 1X SsoFast EvaGreen Supermix, $0.25 \mu \mathrm{M}$ of both forward and reverse primer. See table 2 for primer sequences. Samples were loaded in duplicate into the Hard-Shell ${ }^{\circledR}$ Low-Profile Thin Wall 96 skirted PCR plates (Bio-Rad Cat No: HSP9601), and sealed with the Microseal ${ }^{\circledR}$ 'B' adhesive seals (Bio-Rad Cat No: MSB1001). The PCR plate was spun at $1000 \mathrm{XG}$ for 1 minute on the Sorval Legend RT (Rotor ID: Heraeus Cat No: 75006445, PCR Plate Adaptor: Thermo Scientific Cat No: 75006449R, Rubber Adaptor: Thermo Scientific Cat No: EPDM 20056846). The RT-qPCR was performed on the Bio-Rad CFX1000 with the SYBR green channels selected. The RT-qPCR conditions are as follows: $95^{\circ} \mathrm{C}$ for 3 minutes, followed by 40 cycles of $95^{\circ} \mathrm{C}$ for 10 seconds, $60^{\circ} \mathrm{C}$ for 30 seconds, and $72^{\circ} \mathrm{C}$ for 30 seconds. This step was followed by a melt curve protocol: $95^{\circ} \mathrm{C}$ for 10 seconds, followed by $5^{\circ} \mathrm{C}$ temperature increments starting from $65^{\circ} \mathrm{C}$ and ending at $95^{\circ} \mathrm{C}$. Relative gene expression as fold change normalized to Gapdh, U6, Hprt, or Srp14 was calculated using the $2^{-\Delta \Delta \mathrm{Ct}}$ method. 


\subsection{Muscle histology}

The tibialis anterior (TA) were embedded in Tissue-Tek Optimal Cutting Temperature (OCT) compound (Sakura Cat No: 4583) and snap frozen in liquid $\mathrm{N}_{2}$ cooled 2-methylbutane for approximately 30 seconds (Sigma Cat No: M32631-1L). Embedded tissue was stored at $-80^{\circ} \mathrm{C}$ until further analysis. Transverse sections of the midbelly region of the TA were cut using the Leica CM 1950 cryostat and mounted to Superfrost Plus glass microscopy slides (Fisher Scientific Cat No: 12-550-15). The thickness of the sections was $10 \mu \mathrm{m}$. Slides were stored at $-80^{\circ} \mathrm{C}$ until further analysis. Prior to Hematoxylin and eosin (H\&E) staining, the slides were first brought to room temperature and then carefully rinsed in distilled water or PBS. The slides were then stained in hematoxylin (Merck Cat No: 1.05174.1000) for 1 minute and then rinsed with tap water. The section was then incubated in Scotts tap water for 2 minutes. The composition of Scotts tap water was as follows: $41.7 \mathrm{mM} \mathrm{NaHCO}_{3}$ (Sigma Cat No: S7277), $81.1 \mathrm{mM} \mathrm{MgSO}_{4} .7 \mathrm{H}_{2} \mathrm{O}$ (Sigma Cat No: M1880), prepared in autoclaved MilliQ water and filter sterilized using either $250 \mathrm{ml}$ (Corning Cat No: 431096) or $500 \mathrm{ml}$ (Thermo Scientific Cat No: 566-0020) PES membrane filter units. After a brief rinse with distilled water, the section was then stained with eosin (Merck Cat No: 1.09844.1000) for 30 seconds to 2 minutes, and then rinsed with distilled water. The section was then sequentially dehydrated in ethanol. First, the section was dipped into $50 \%$ ethanol and then $70 \%$ ethanol. The section was then incubated in $95 \%$ ethanol for 2 minutes, and repeated once again with fresh $95 \%$ ethanol. This procedure was repeated with $100 \%$ ethanol. The slides were next incubated in xylene (Sigma Cat No: 5340564) for 5 minutes, and repeated once again for 5 minutes with fresh xylene. The slides were mounted with a coverslip (CellPath Cat No: SAh-2450-03A) and DPX mounting media (Merck Cat No: 1.01979.0500). Slides were stored at room temperature. Images were captured using the Leica CTR 6500 microscope, equipped with the Leica DFC 420 FX camera (Leica) and Image-Pro Plus 6 software (Media Cybernetics). 


\subsection{Myofiber Fiber Area}

H\&E stained TA sections were used to calculate muscle fiber cross-sectional area (CSA). The area of approximately 350 myofibers per mouse (over 4 random fields of view) was estimated. Please note that the entire TA was first tiled at 5X. In some instances, the myofiber area of the TA was counted in duplicate, and subsequently averaged. Image Pro Plus software (Media Cybernetics) was used to estimate the myofiber area.

\subsection{Intestine length and weight measurement}

After the intestine was carefully removed from the mouse, followed by the debridement of the mesenteric adipose tissue and the cecum, the intestinal segments were placed onto a filter paper. The small intestine was cut into 3 or 4 equal portions with their directionality maintained. The lengths of the intestines were measured using a Vernier caliper. The uncleared weight of the small and large intestines were also recorded. To measure the cleared weight, the luminal contents were expelled by carefully squeezing the intestine.

\subsection{Serum Preparation and Myostatin ELISA}

Mouse serum was obtained by cardiac puncture method using a 26 (1/2") G needle (BD Cat No: 302002). Blood was clotted at room temperature for 30 minutes, followed by centrifugation at $1,500 \mathrm{XG}$ for 15 minutes at $4^{\circ} \mathrm{C}$ (Eppendorf 5415R - FA-45-24-11, 04874). The clarified serum was aliquoted into fresh microcentrifuge tubes and stored at $-80^{\circ} \mathrm{C}$ until further analysis. Myostatin serum levels were measured by either the Myostatin ELISA kit by Immundiagnostik (Cat No: K1012) or the GDF8/Myostatin Quantikine ELISA Kit by R\&D Systems (Cat No: DGDF80). The experiment was performed according to the protocol provided by the manufacturer.

\subsection{Statistical analysis and figure preparation}

Statistical analysis was performed using the GraphPad Prism (Version 6.07). Graphs were prepared using GraphPad Prism. Composite images were prepared using Adobe Illustrator CS6. 
Table 3.1: Antibodies and the immunoblotting conditions.

\begin{tabular}{|c|c|c|c|c|c|}
\hline Antibody & Catalogue Number & Company & Conditions & Incubation Time & Dilution \\
\hline MuRF1 & Gift & Regeneron & $5 \%$ Milk in TBST & Overnight at $4^{\circ} \mathrm{C}^{\dagger}$ & $1: 200,1: 500$ \\
\hline Atrogin-1 & PAB15627 & Abnova & $5 \%$ Milk in TBST & Overnight at $4^{\circ} \mathrm{C}$ & $1: 500$ \\
\hline FoxO3 & 2497 & $\begin{array}{c}\text { Cell Signaling } \\
\text { Technology }\end{array}$ & $5 \%$ BSA in TBST & Overnight at $4^{\circ} \mathrm{C}$ & $1: 1000$ \\
\hline Phospho-FoxO3 (Ser 318/321) & 9465 & $\begin{array}{l}\text { Cell Signaling } \\
\text { Technology }\end{array}$ & $5 \%$ BSA in TBST & Overnight at $4^{\circ} \mathrm{C}$ & $1: 1000$ \\
\hline Cleaved Caspase-3 & 9661 & $\begin{array}{c}\text { Cell Signaling } \\
\text { Technology }\end{array}$ & $5 \%$ BSA in TBST & Overnight at $4^{\circ} \mathrm{C}$ & $1: 1000$ \\
\hline Myostatin & MAB788 & R\&D Systems & $5 \%$ BSA in TBST & Overnight at $4^{\circ} \mathrm{C}$ & $1: 500$ \\
\hline Myostatin & 98337 & Abcam & $5 \%$ BSA in TBST & Overnight at $4^{\circ} \mathrm{C}$ & $1: 500$ \\
\hline GAPDH & 32233 & Santa Cruz & 5\% Milk TBST & $\begin{array}{l}1 \text { hour at room } \\
\text { temperature }\end{array}$ & $1: 10,000,1: 20,000$ \\
\hline Anti-Rabbit HRP & $170-6515$ & Bio-Rad & $5 \%$ Milk TBST & $\begin{array}{c}1 \text { hour at room } \\
\text { temperature }\end{array}$ & $1: 5000$ \\
\hline Anti-Mouse HRP & $170-6516$ & Bio-Rad & $5 \%$ Milk TBST & $\begin{array}{c}1 \text { hour at room } \\
\text { temperature }\end{array}$ & $1: 5000,1: 10,000$ \\
\hline Anti-Goat HRP & 2768 & Santa Cruz & $5 \%$ Milk TBST & $\begin{array}{c}1 \text { hour at room } \\
\text { temperature }\end{array}$ & $1: 2500$ \\
\hline Anti-Rat HRP & 2006 & Santa Cruz & 5\% Milk TBST & $\begin{array}{c}1 \text { hour at room } \\
\text { temperature }\end{array}$ & $1: 2500,1: 5000$ \\
\hline
\end{tabular}

$\dagger$ Immunoblot represented in Figure 4.3.2A and Figure 7.3.6 was incubated for 2 days at $4^{\circ} \mathrm{C}$.

$\$$ Immunoblot represented in Figure 7.3.7 was incubated for 2 days at $4^{\circ} \mathrm{C}$. 
Table 3.2: Primers.

$\underline{\text { RT-qPCR }}$

\begin{tabular}{|c|c|c|}
\hline & Forward & Reverse \\
\hline Atrogin-1 & TTCAGCAGCCTGAACTACGAC & TTGAAAGCTTCCCCCAAAGTA \\
\hline Murf1 & TACCAAGCCTGTGGTCATCC & CCGGTCCATGATCACTTCAT \\
\hline Mstn & ACGGAAACAATCATTACCATGC & TAGGAGTCTTGACGGGTCTGAG \\
\hline Mki67 & AGCTAACTTGCGCTGACTGG & CAAACAGGCAGGAGCTGAGG \\
\hline Pcna & CTCGTCTCACGTCTCCTTGG & GGACATGCTGGTGAGGTTCA \\
\hline $\operatorname{Lgr} 5$ & CTGCCCATCACACTGTCACT & GCAGAGGCGATGTAGGAGAC \\
\hline Bmil & CGCTTGGCTCGCATTCATTT & TACCCTCCACACAGGACACA \\
\hline Il6 & GCCTTCTTGGGACTGATGCT & TGCCATTGCACAACTCTTTTCT \\
\hline Tnfalpha_variant 1 & CCCACGTCGTAGCAAACCA & ACAAGGTACAACCCATCGGC \\
\hline Tnfalpha_variant 1 & AGGCACTCCCCCAAAAGATG & CCACTTGGTGGTTTGTGAGTG \\
\hline Ifngamma & GGCAAAAGGATGGTGACATGAA & TTTTCGCCTTGCTGTTGCTG \\
\hline Gapdh & AGCTTGTCATCAACGGGAAG & CGGAGATGATGACCCTTTTG \\
\hline Srp 14 & CCGAGGAAGAGTTCTGTGGAG & CGTCCATGTTGGCTCTCAGTA \\
\hline Hprt & CCAGACAAGTTTGTTGTTGGA & TTTACTAGGCAGATGGCCACA \\
\hline U6 & CGCTTCGGCAGCACATATAC & CGAATTTGCGTGTCATCCTT \\
\hline \multicolumn{3}{|c|}{ Genotyping } \\
\hline$M s t n^{-/-}$ & Forward & Reverse \\
\hline mMstn_Geno & CAGCCATGGTAGTAGACCG & GATGTGCTCTCACTTCCTTG \\
\hline mNeo_Geno & TCTATCGCCTTCTTGACGAG & GATGTGCTCTCACTTCCTTG \\
\hline Mstn1 & GGCATCTGTTCTGCTATTACGTGC & $\mathrm{N} / \mathrm{A}$ \\
\hline Mstn2 & GTGCGATAATCCAGTCCCAT & $\mathrm{N} / \mathrm{A}$ \\
\hline Mstn3 & GTGGATGTGGAATGTGTGCGAGG & $\mathrm{N} / \mathrm{A}$ \\
\hline$A p c^{\operatorname{Min} /+}$ & Primer & End Label \\
\hline ApcMin_13779 & GGGAAGTTTAGACAGTTCTCGT & $\mathrm{N} / \mathrm{A}$ \\
\hline ApcMin_13780 & TGTTGGATGGTAAGCACTGAG & $\mathrm{N} / \mathrm{A}$ \\
\hline ApcMin_13781 & AGACAGAAGTTAGGAGAGAGAGC & 5' FAM 3' BHQ1 \\
\hline ApcMin_13782 & AGACAGAAGTTTGGAGAGAGAGC & 5' JOE 3' BHQ1 \\
\hline \multicolumn{3}{|c|}{$\underline{\text { Cloning }}$} \\
\hline & Forward & Reverse \\
\hline sActRIIB $(A c v r 2 b)$ & GAGGATCCGTCCGGGCGAGGGGAGGCT & GGATCCTCACGTGAGCAGGGTGGGGGC \\
\hline
\end{tabular}




\section{Results}

\section{1 $\quad A p c^{M i n /+}$ mice demonstrated cancer cachexia}

The $A p c^{\mathrm{Min} /+}$ mouse has been extensively used to study intestinal and colonic tumorigenesis (see [265] for review). The $\mathrm{Apc}^{\mathrm{Min} /+}$ mouse is also gaining appreciation as a model that mimics cancer cachexia $[138,253]$. To verify that the $A p c^{M i n /+}$ mouse exhibits the symptoms of cancer cachexia, we first examined the weekly body weights of male $A p c^{\mathrm{Min} /+}$ mice (Figure 4.1.1). Female mice were not included in this study as they are also susceptible to the development of mammary carcinomas, thus confounding our investigation into the study of myostatin and the onset of intestinal tumors [254]. Male $A p c^{M i n /+}$ mice displayed a similar growth rate to wild type mice from 5 to 7 weeks (Figure 4.1.1 and Table 7.1.1). Male $A p c^{\mathrm{Min} /+}$ mice started to demonstrate a significant change in body weight compared to wild type mice as early as 8 weeks. After 14 weeks, male $A p c^{\mathrm{Min} /+}$ mice exhibited a rapid decrease in body mass that continued till 20 weeks (Figure 4.1.1). However, this decrease in mass observed in male $A p c^{\mathrm{Min} /+}$ mice was heterogeneous (Figure 4.1.1 and Table 7.1.1). The large variation observed at 16 to 20 weeks of age was partly due to some $A p c^{M i n /+}$ mice that exhibited a body weight similar to wild type age matched controls (Figure 4.1.1 and Table 7.1.1). It must be noted that the variable number of mice use for this experiment was largely contributed by drop-outs due to death or termination of mice after the age of 18 weeks for the various experiments described in this report, and drop-ins due to our initial purchase of $\mathrm{Apc}^{\mathrm{Min} /+}$ mice from the Jackson Laboratories that were used to first establish our colony. 


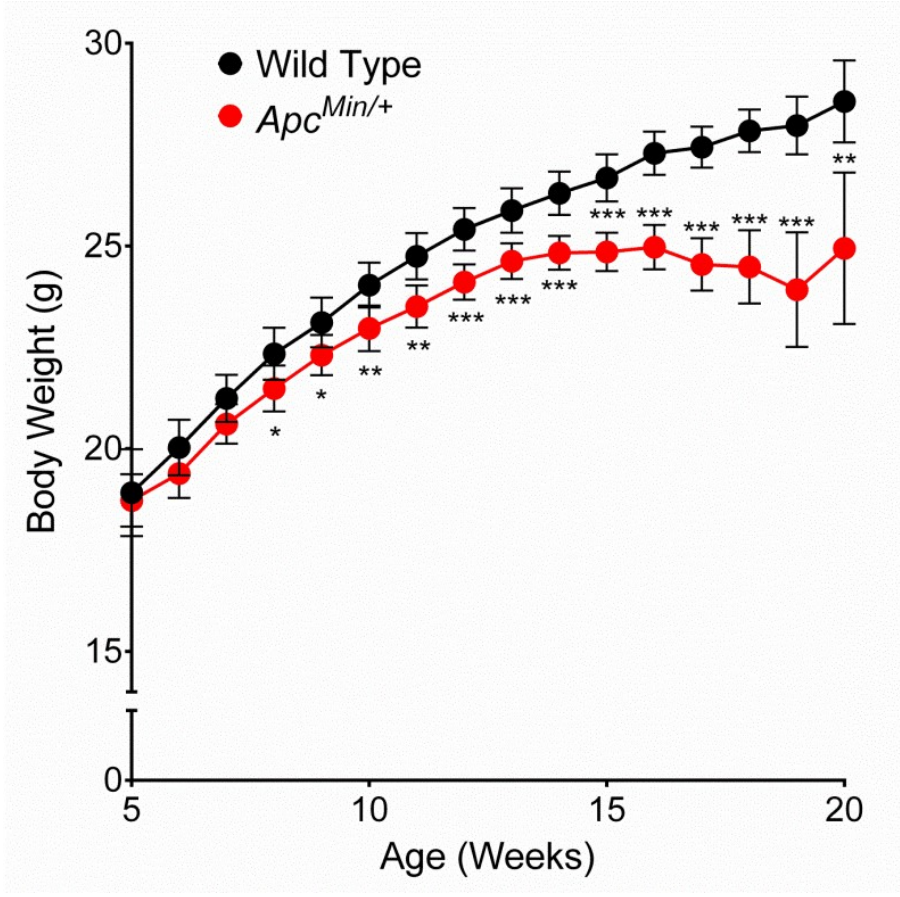

Figure 4.1.1: Aging male $A p c^{M i n /+}$ mice displayed body weight loss.

Weekly body weights for male $A p c^{M i n /+}$ mice (n=9-50) and wild type controls (n=11-38) measured from 5 to 20 weeks. Significance was assessed by Student's t-test with Welch correction: ${ }^{*} \mathrm{p}<0.05$, $* * p<0.01, * * * p<0.001$. Please refer to Table 7.1 .1 for supplementary information pertaining this experiment. Error bars denote $\pm 95 \%$ CI. 
The variability in weight loss observed in aging male $A p c^{\mathrm{Min} /+}$ mice prompted us to adopt an alternative strategy to characterize the progression of cancer cachexia in this particular model. We chose to determine the progression of cancer cachexia as a function of body weight loss rather than the age of the $A p c^{\mathrm{Min} /+}$ mouse. Thus, using a similar strategy devised by Baltglavis and colleagues, we characterized the severity of cancer cachexia by sacrificing the $A p c^{\mathrm{Min} /+}$ mice according to their percentage body weight loss $[137,279]$. The percentage body weight loss was calculated by dividing the live body weight at the time of sacrifice to the peak body weight of the male $\mathrm{Ap} \mathrm{C}^{\mathrm{Min} /+}$ mouse. This methodology was chosen to circumvent the variation in body weight that would have occurred by terminating the male $A p c^{\mathrm{Min} /+}$ mice according to their age [279].

The $A p c^{M i n /+}$ mice were stratified into four groups based on the extent of weight loss (refer to Table 7.1.2 for a detailed description of each mouse used for the experiment). $\mathrm{Apc}^{\mathrm{Min} /+}$ mice that demonstrated approximately $4.8 \%$ to $7.2 \%$ decrease in their live body weight when compared to their peak body weight were grouped as early cachexia $(n=5)$ (Table 4.1.1 and Table 7.1.2). Intermediate level of cachexia consists of $\mathrm{Apc}^{\mathrm{Min} /+}$ mice that displayed a percentage body weight loss of $9.8 \%$ to $14 \%(n=4)$ (Table 4.1 .1 and Table 7.1.2). $A p c^{M i n /+}$ mice that lost over $20 \%$ of their body weight were segregated into the severe cachexia group $(n=6)$ (Table 4.1.1 and Table 7.1.2). $A p c^{\mathrm{Min} /+}$ mice that demonstrated no change in their percentage body weight were grouped as noncachectic $(n=7)$ (Table 4.1.1 and Table 7.1.2). 14 week old wild type mice were also used for this experiment $(n=6)$ (Table 4.1.1). The age of the mice used for this trial spanned from 14 to 18 weeks of age, except for two mice in the severe cachexia group (mouse ID 415 and 902) which were approximately 19 and 22 weeks old, respectively (Table 4.1.1 and Table 7.1.2). The tibia lengths for these four groups of mice were similar to the wild type controls (Table 7.1.3). 
Table 4.1.1: Body weights of $A p c^{M i n /+}$ mice stratified by their percentage body weight loss

\begin{tabular}{lccccc} 
& & \multicolumn{4}{c}{$A p c^{\text {Min } /+}$} \\
\cline { 3 - 6 } & Wild Type & Non-Cachectic & Early & Intermediate & Severe \\
\hline Peak body weight $(\mathrm{g})$ & N/A & N/A & 26.2 & 26.1 & 25.4 \\
\pm S.D. & N/A & N/A & 0.4 & 1.1 & 1.9 \\
\hline Sacrificed body weight $(\mathrm{g})$ & 26.2 & 25.7 & $24.7^{* * *}$ & $22.6^{* * *+}$ & $19.3^{* * * *}+$ \\
\pm S.D. & 0.7 & 1.0 & 0.6 & 0.6 & 1.4 \\
\hline Percentage change & & & 5.7 & 13.7 & 24.1 \\
\hline
\end{tabular}

*** Peak body weight significantly different to sacrificed body weight $(\mathrm{p}<0.001)$ as determined by a twotailed paired Student's t-test

¥ Sacrificed body weight significantly different to non-cachectic sacrificed body weight $(\mathrm{p}<0.001)$ as determined by a one-way ANOVA followed by Tukey's post-hoc test 


\subsection{Cachectic $\mathrm{Apc}^{\mathrm{Min} / \mathrm{+}}$ mice displayed skeletal muscle and adipose tissue atrophy}

The loss of skeletal muscle mass is a hallmark of cancer cachexia [284]. While there was no evidence of weight loss in the gastrocnemius (GAS) and quadricep (QUAD) muscles collected from the early cachexia group when compared to the non-cachectic group, the atrophy of these

particular muscles became more pronounced as the $\mathrm{Apc}^{\mathrm{Min} /+}$ mouse developed a severe cachectic phenotype (Figure 4.2.1A and Table 7.2.1). Similarly, the extensor digitorum longus (EDL) exhibited a similar decrease in muscle weight as the severity of cancer cachexia increased (Table 7.2.1). The weights of the soleus (SOL), a muscle possessing mostly slow-twitch fibers, were relatively stable across the four cachexia groups (Table 7.2.1). Additionally, there appeared to be no difference in the muscle mass of the wild type and non-cachectic $A p c^{\mathrm{Min} /+}$ mice (Figure 4.2.1A and Table 7.2.1).

The tibialis anterior (TA) weights from the early and intermediate cachectic groups were similar to the non-cachectic controls (Table 7.2.1). However, analysis of the myofiber cross-sectional area of the TA from the early and moderately cachectic $\mathrm{Apc}^{\mathrm{Min} /+}$ mice revealed a significantly greater percentage of smaller, atrophied myofibers $\left(\geq 1000\right.$ to $\left.<2000 \mu \mathrm{m}^{2}\right)$, and a smaller proportion of large myofibers $\left(\geq 3000\right.$ to $<4000 \mu \mathrm{m}^{2}$ ) compared to the non-cachectic controls (Figures 4.2.1B and 4.2.1C). This preponderance of these smaller myofibers was more apparent in the severely cachectic group, further confirming the involvement of muscle atrophy during the progression of cancer cachexia in the $\mathrm{Apc}^{\mathrm{Min} /+}$ mouse (Figures 4.2.1B and 4.2.1C). In addition, there was no significant difference in the myofiber area between the non-cachectic mice compared to the wild type controls (Figures 4.2.1B and 4.2.1C). 
A)

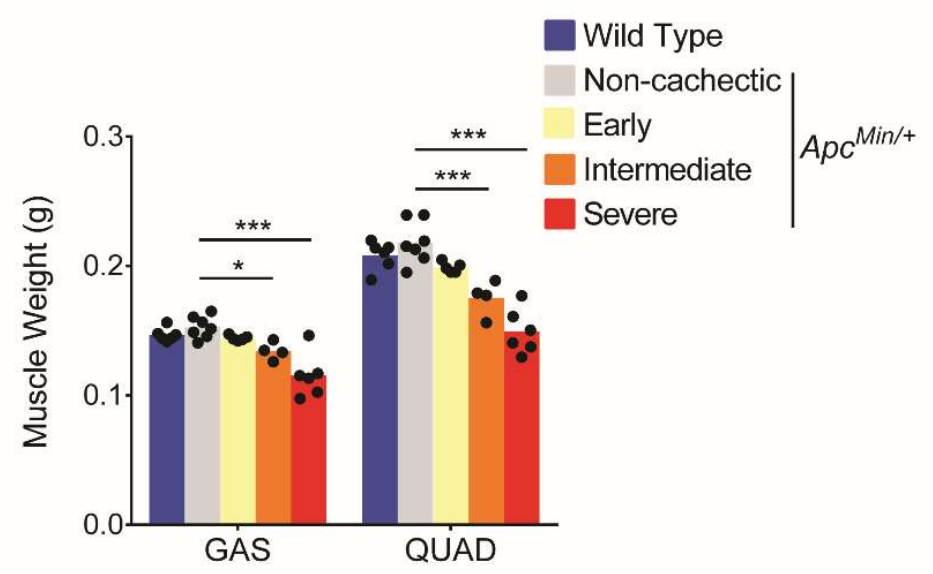

B) $\quad \mathrm{Apc}^{\mathrm{Min} /+}$

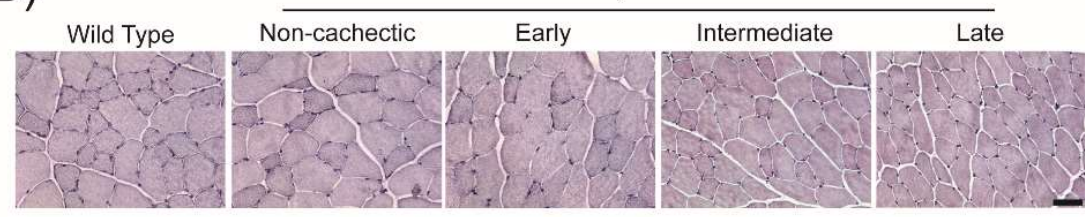

C)

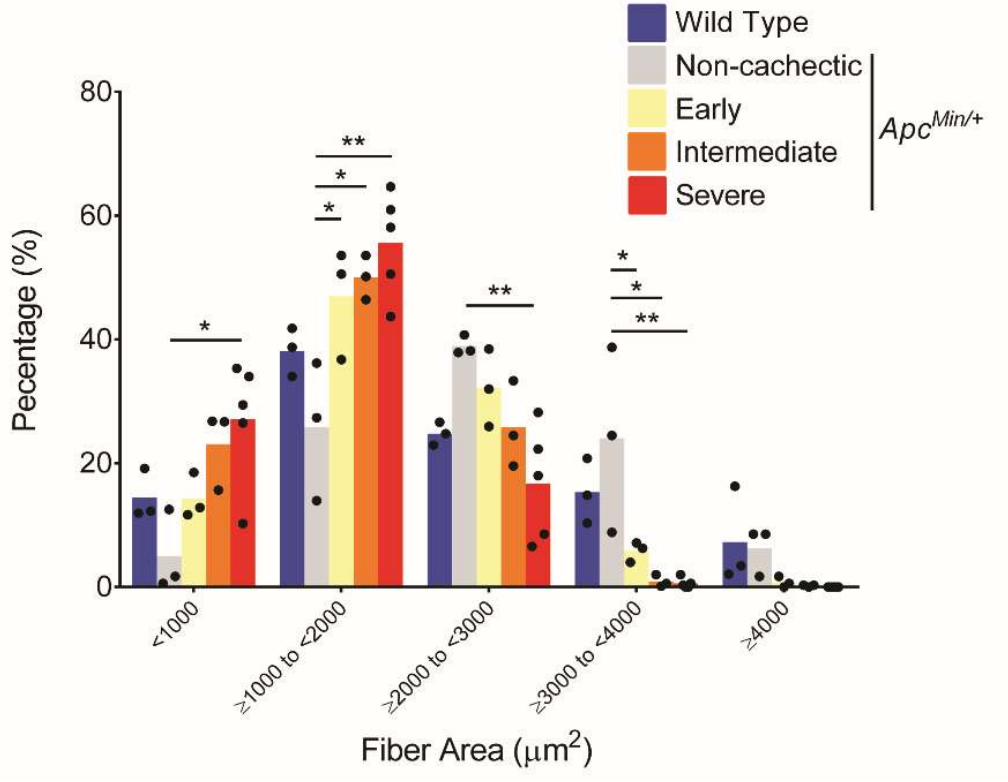

Figure 4.2.1: Cachectic $\mathrm{Apc}^{\mathrm{Min} /+}$ mice demonstrated skeletal muscle atrophy.

A) Gastrocnemius (GAS) and quadricep (QUAD) weights (grams) for wild type (n=6), non-cachectic $(\mathrm{n}=7)$, early $(\mathrm{n}=5)$, intermediate $(\mathrm{n}=4)$, and severely $(\mathrm{n}=6)$ cachectic $A p c^{\mathrm{Min} /+}$ mice. Each data point represents the average weight of the muscle collected from the two hind limbs of the mouse. The right QUAD weight from Mouse ID 907 (Early) was represented in this figure as there was an error in the collection of the left QUAD. Significance was assessed by one-way ANOVA followed by Tukey's post-hoc test: ${ }^{*} \mathrm{p}<0.05, * * \mathrm{p}<0.01, * * * \mathrm{p}<0.001$.

B) Representative H\&E images of cryosectioned tibialis anterior (TA). Scale bar represents $50 \mu \mathrm{m}$.

C) Percentage frequency distribution of the TA myofiber cross-sectional area $\left(\mu \mathrm{m}^{2}\right)$ in the wild type $(n=3)$, non-cachectic $(n=3)$, early $(n=3)$, intermediate $(n=3)$, and severely $(n=5)$ cachectic $A p c^{\text {Min } /+}$ mice. Significance was assessed by one-way ANOVA followed by Tukey's post-hoc test: ${ }^{*} p<0.05$, $* * \mathrm{p}<0.01, * * * \mathrm{p}<0.001$. 
In addition to the atrophy of the skeletal muscle, cancer cachexia can be associated with the loss of adipose tissue mass [6]. Indeed, a significant reduction in both the inguinal and epididymal adipose tissue depots were observed in the severely cachectic $\mathrm{Apc}^{\mathrm{Min} /+}$ mice when compared to the non-cachectic controls (Figure 4.2.2). The early and intermediate cachexia groups did not demonstrate a significant reduction in the inguinal and epididymal adipose tissue weights (Figure 4.2.2). Furthermore, the retroperitoneal adipose tissue depot remained relatively unchanged during the progression of cancer cachexia in the $\mathrm{Apc}^{\mathrm{Min} / \mathrm{+}}$ mouse (Figure 4.2.2). According to Figure 4.2.2, it is important to note that there was a higher amount of adiposity particularly in the inguinal adipose tissue depots of the wild type mice compared to the non-cachectic $A p c^{\mathrm{Min} /+}$ mice (Figure 4.2.2).

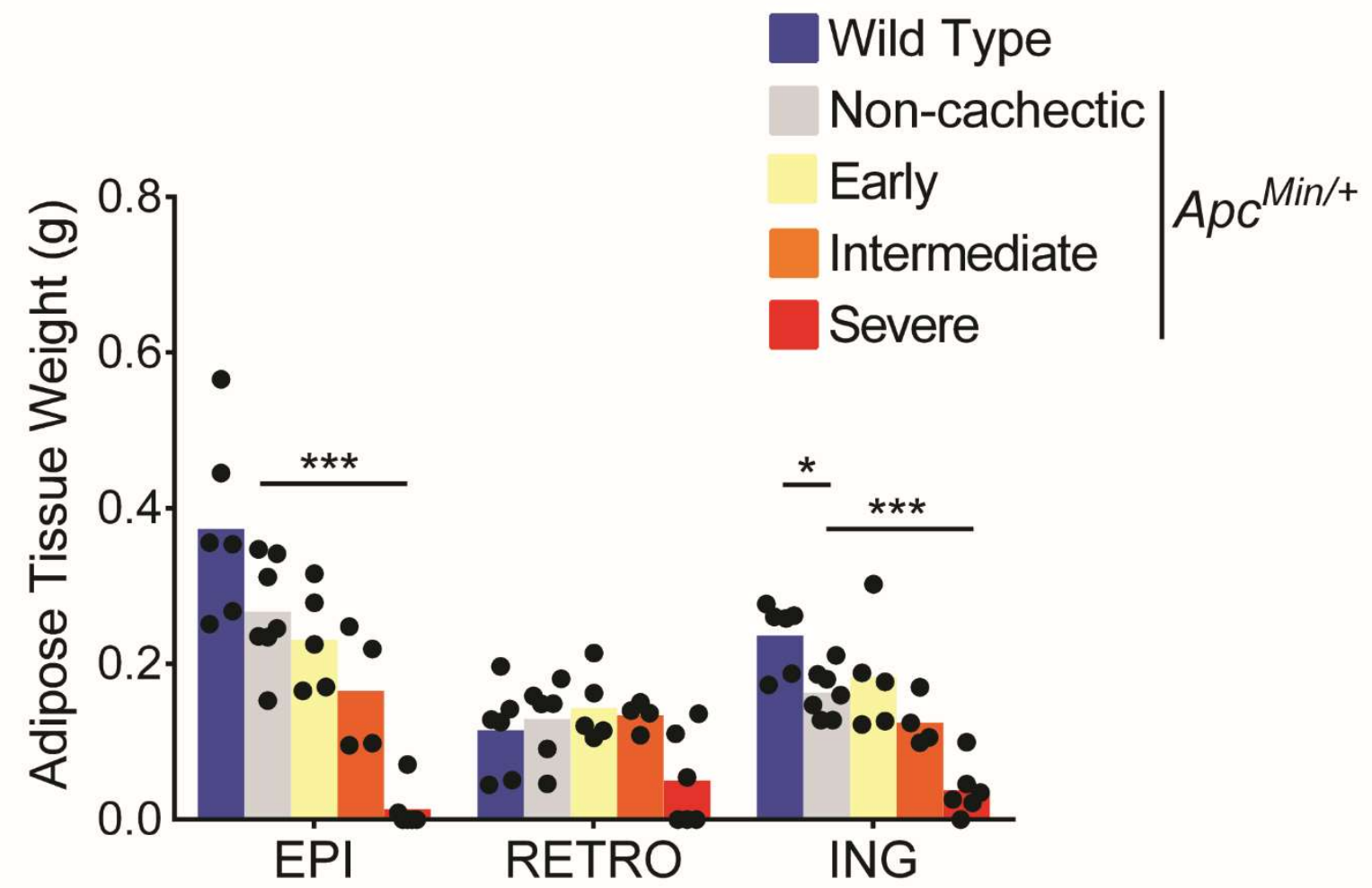

Figure 4.2.2: Cachectic $A p c^{M i n /+}$ mice exhibited adipose tissue atrophy.

Epididymal (EPI), retroperitoneal (RETRO), and inguinal (ING) adipose tissue weights (grams) for wild type $(n=6)$, non-cachectic $(n=7)$, early $(n=5)$, intermediate $(n=4)$, and severely $(n=6)$ cachectic $A p c^{\mathrm{Min} /+}$ mice. Each data point represents the average weight of the two adipose tissue depots. RETRO weight of Mouse ID 412 (Non-cachectic) was omitted as there was an error in its measurement ( 0.905 grams). Significance was assessed by one-way ANOVA followed by Tukey's post-hoc test: ${ }^{*} p<0.05, * * p<0.01, * * * p<0.001$. 


\subsection{Skeletal muscle atrophy in cachectic $\mathrm{Apc}^{\mathrm{Min} / \mathrm{+}}$ mice was associated with the increased expression of the ubiquitin-proteasome pathway}

Elevated expression and activity of the ubiquitin-proteasome pathway (UPP) is a salient feature of skeletal muscle atrophy [62]. In particular, the upregulation of MuRF1 and Atrogin-1, two muscle specific E3 ligases which aid in the degradation of myofibrillar proteins, is an established marker of the skeletal muscle atrophy program [67]. Indeed, a dramatic increase in both the gene and protein expression of Atrogin-1 and MuRF1 were observed in the intermediate and severely cachectic $A p c^{\mathrm{Min} / \mathrm{+}}$ mice when compared to the non-cachectic controls (Figures 4.3.1, 4.3.2, 7.3.1, 7.3.3, 7.3.6, and 7.3.7). The upregulation of the muscle specific E3 ligases is regulated by the FoxO transcription factors [116]. To assay the involvement of FoxO3 during the progression of cancer cachexia, immunoblots for the inactive, phosphorylated form of FoxO3 (p-FoxO3) and total amounts of FoxO3 were performed on the four $\mathrm{Apc}^{\mathrm{Min} /+}$ cachexia groups (Figures 4.3.2A, 4.3.2B, and Figures 7.3.27.3.5). While there was no observable change in $\mathrm{p}-\mathrm{FoxO} 3$ amounts during the initial stages of cachexia, the proportion of inactive p-FoxO3 reduced significantly as the $\mathrm{Apc}^{\mathrm{Min} /+}$ mice progressively lost their body weight (Figures 4.3.2A, 4.3.2B, and Figures 7.3.2-7.3.5). Taken together, increased expression of the UPP is associated with the increasing severity of cachexia in the $A p c^{\mathrm{Min} /+}$ mouse. 


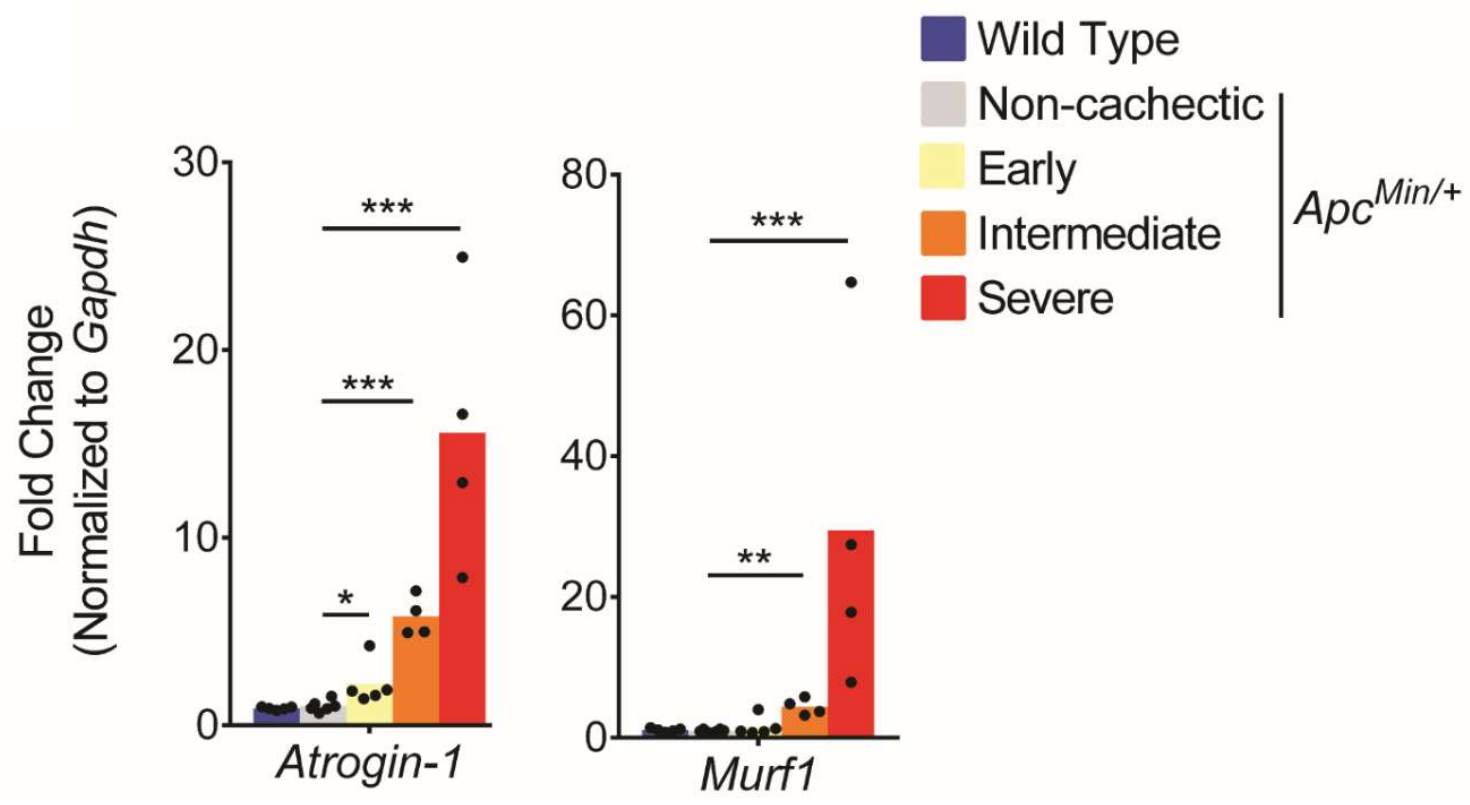

Figure 4.3.1: Increased levels of Atrogin-1 and Murf1 mRNA was associated with increasing severity of cancer cachexia in the $A \mathrm{pc}^{\mathrm{Min} / \mathrm{+}}$ mouse.

Relative gene expression of Atrogin- 1 and Murfl in the GAS muscles of wild type ( $\mathrm{n}=5)$, non-

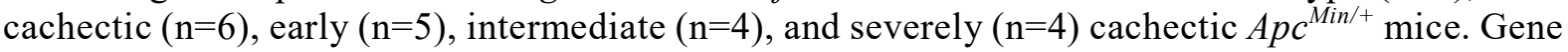
expression was normalized to Gapdh. Significance was assessed by one-way ANOVA followed by Tukey's post-hoc test: ${ }^{*} \mathrm{p}<0.05, * * \mathrm{p}<0.01, * * * \mathrm{p}<0.001 . \Delta \mathrm{Ct}$ was used to calculate significance. 

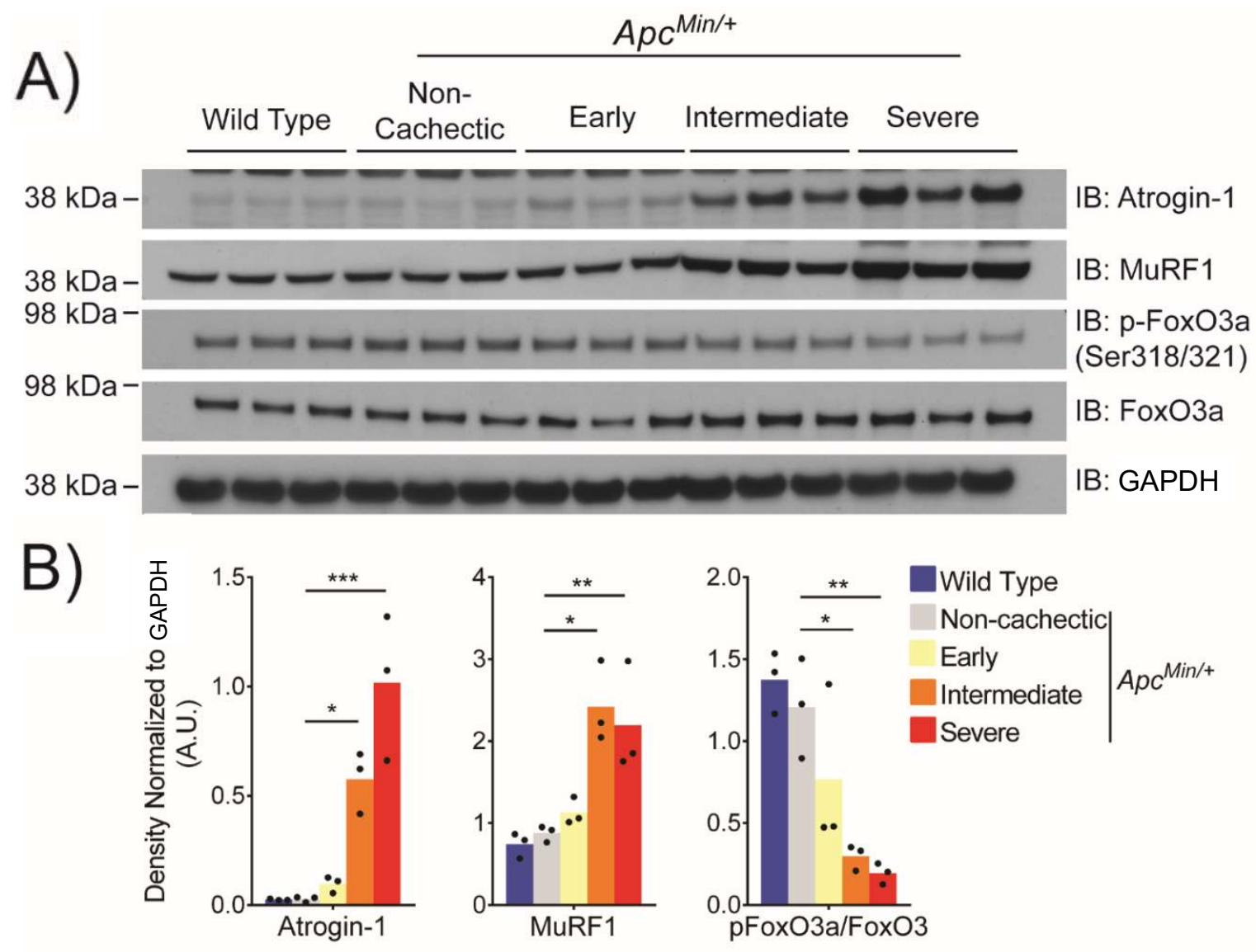

Figure 4.3.2: Elevated protein expression of key mediators of the ubiquitin-proteasome pathway was associated with increasing severity of cancer cachexia in the $\mathrm{Apc}^{\mathrm{Min} / \mathrm{+}}$ mouse. A) Immunoblots for Atrogin-1, MuRF1, phosphorylated FoxO3a (p-FoxO3a Ser318/321), total FoxO3a for the GAS muscles of wild type, non-cachectic, early, intermediate, and severely cachectic $\operatorname{Apc}^{\mathrm{Min} /+}(\mathrm{n}=3)$ mice. GAPDH was used as a loading control. GAPDH shown here corresponds to the loading control used for both Atrogin-1 and p-FoxO3a, as this same immunoblot was used to detect for the presence of these two proteins (Figure 7.3.3). The molecular weight markers are represented on the left side of the immunoblots. The immunoblots used for this figure and their corresponding GAPDH loading controls are displayed in Figures 7.3.1-7.3.7. B) Densitometry for Atrogin-1 and MuRF1 normalized to their corresponding GAPDH loading controls for immunoblots represented in Figure 4.3.2A. Densitometry for pFoxO3a and FoxO3a normalized to their corresponding GAPDH loading controls and represented as a ratio. Significance was assessed by one-way ANOVA followed by Tukey's post-hoc test: ${ }^{*} \mathrm{p}<0.05$, $* * \mathrm{p}<0.01, * * * \mathrm{p}<0.001$ 


\subsection{Myostatin gene expression was elevated in the skeletal muscles of cachectic $\mathrm{Apc}^{\mathrm{Min} /+}$ mice}

Myostatin is a TGF- $\beta$ superfamily member growth hormone that negatively regulates skeletal muscle mass [150]. The over-expression of myostatin in mice leads to severe skeletal muscle atrophy [144]. Conversely, the genetic ablation of this growth factor leads to the hypertrophy and hyperplasia of the skeletal muscle $[140,142]$. Given that the $\mathrm{Apc}^{\mathrm{Min} /+}$ mice displayed skeletal muscle wasting, we hypothesized that the cancer cachexia in the $A p c^{\mathrm{Min} /+}$ mouse is manifested by the increased expression of myostatin. Indeed, myostatin transcript levels were elevated to over 1.5 to 2.5 fold in the GAS muscles of the intermediate cachexia group compared to the noncachectic control (Figure 4.4.1 and Figure 7.4.1A). The elevated myostatin gene expression levels were also observed in the severely cachectic $A p c^{\mathrm{Min} /+}$ mice (Figure 4.4.1). $A p c^{\mathrm{Min} /+}$ mice that demonstrated the early signs of cachexia did not display any significant increase in myostatin gene expression when compared to the non-cachectic control (Figure 4.4.1 and Figure 7.4.1).

It must be noted that the severe cachexia group demonstrated a large variation in myostatin gene expression despite our measures to carefully stratify the body weight loss exhibited by the $\mathrm{Apc}$ Min/+ mice (Figure 4.4.1). This variation seen in the severe cachexia group resulted in one replicate of this experiment failing to display any significant difference to the non-cachectic controls (Figure 7.4.1F). To further validate the observation that $\mathrm{Apc}^{\mathrm{Min} /+}$ mice undergoing intermediate to severe cachexia exhibited a significant increase in myostatin gene expression, two more endogenous controls, Hprt and Srp14, were included in our analysis. The use of both Hprt and Srp14 as an endogenous control resulted in a noticeable increase in the myostatin gene expression levels in the intermediate cachexia group ( $>1.5$ fold), the latter displayed a significant difference when compared to the non-cachectic $\mathrm{Apc}^{\mathrm{Min} / \mathrm{+}}$ controls (Figure 7.4.2). The myostatin gene expression in the severe cachexia group was similar to the non-cachectic controls when Hprt and Srp14 were used as endogenous controls (Figure 7.4.2). 


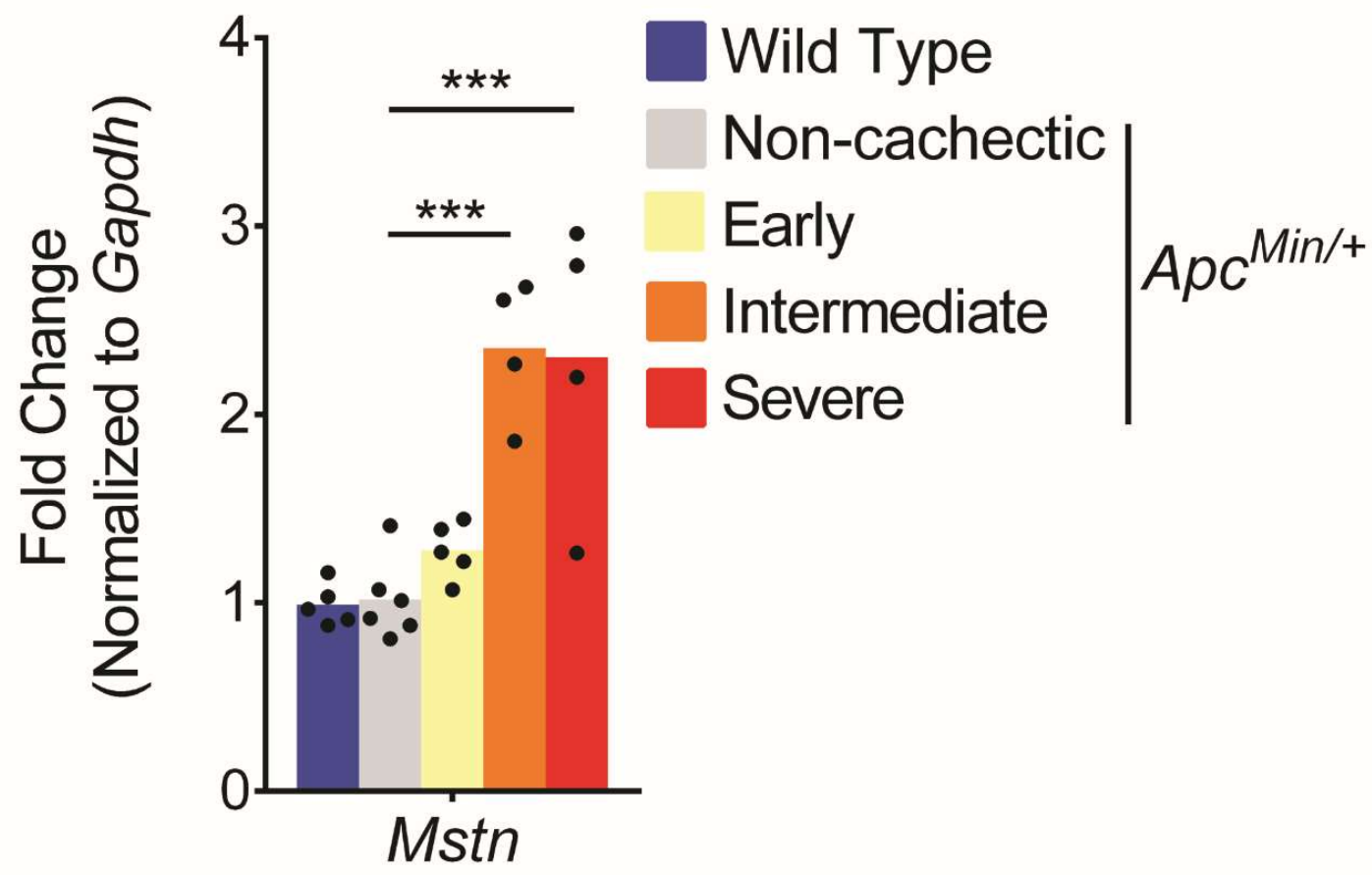

Figure 4.4.1: Myostatin gene expression levels were elevated in the GAS muscles of moderately to severely cachectic $A p c^{\mathrm{Min} /+}$ mice.

Relative gene expression of myostatin (Mstn) in the GAS muscles of wild type ( $\mathrm{n}=5)$, non-cachectic $(\mathrm{n}=6)$, early $(\mathrm{n}=5)$, intermediate $(\mathrm{n}=4)$, and severely $(\mathrm{n}=4)$ cachectic $A p c^{\mathrm{Min} /+}$ mice. Gene expression was normalized to Gapdh. Significance was assessed by one-way ANOVA followed by Tukey's post-hoc test: ${ }^{*} \mathrm{p}<0.05,{ }^{* *} \mathrm{p}<0.01, * * * \mathrm{p}<0.001 . \Delta \mathrm{Ct}$ was used to calculate significance. 


\section{5 $A p c^{\mathrm{Min} /+}$ polyps mice express interleukin-6 and TNFa}

The increased expression of myostatin during the intermediate to late stages of cancer cachexia in the $\mathrm{Apc}^{\mathrm{Min} /+}$ mouse was indicative of systemic inflammation. To identify the source of this inflammation, we queried the gene expression of pro-cachectic cytokines in the polyps of $\mathrm{Apc}$ Min/+ mice. According to Figure 4.5.1A, myostatin messenger RNA levels were nearly absent in the small intestine polyps when compared to the skeletal muscle (Figure 4.5.1A). Myostatin gene expression was undetectable in the non-cancerous intestinal tissue and the wild type small intestine control (Figure 4.5.1A). However, polyps in the small intestine of $A p c^{\mathrm{Min} /+}$ mice were enriched with two pro-cachectic cytokines, interleukin-6 (IL-6) and tumor necrosis factor alpha (TNF $\alpha$, variant 1), when compared to both the non-cancerous and wild type intestinal tissue (Figures 4.5.1B and 4.5.1C). The enrichment of IL-6 and TNF $\alpha$ transcripts in the polyps were recapitulated when U6 and Hprt were used as endogenous controls (Figure 7.5.1). TNF $\alpha$ variant 2 messenger RNA levels, on the other hand, were lower in the polyps when compared to normal non-cancerous tissue (Figure 7.5.2). Interferon gamma (IFN $\gamma$ ) was significantly upregulated in the intestinal tissue surrounding the polyps when compared to wild type small intestinal tissue (Figure 4.5.1D).

TNF $\alpha$ and IL-6 are potent inducers of myostatin expression in skeletal muscles [285-287]. Given that the polyps of $\mathrm{Apc}^{\mathrm{Min} /+}$ mice are a rich source of these pro-cachectic inflammatory molecules, we postulated that there could be a correlation between the number of the small intestine tumors and the body weight of the $\mathrm{Apc}^{\mathrm{Min} / \mathrm{+}}$ mice. According to Figure 4.5.2A, we found no relationship between the number of small intestine tumors and the body weight of aged $\mathrm{Apc}^{\mathrm{Min} / \mathrm{+}}$ mice (Figure 4.5.2A). Furthermore, myostatin gene expression in the GAS muscles of these $A p c^{M i n /+}$ mice failed to display any correlation with the small intestine tumor number (Figure 4.5.2B). 


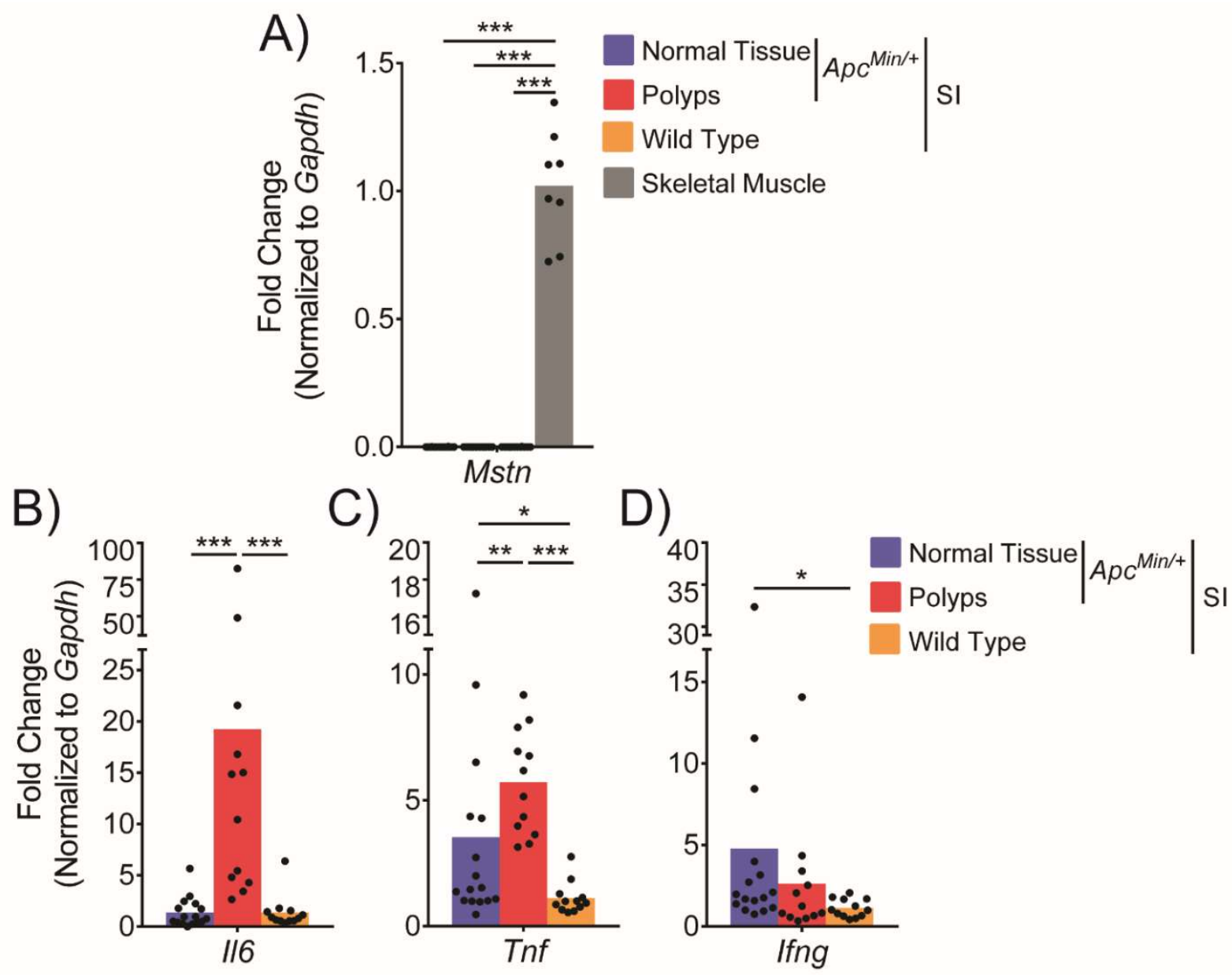

Figure 4.5.1: Small intestine polyps from $\mathrm{Apc}^{\mathrm{Min} / \mathrm{+}}$ mice express IL-6 and TNFa.

A) Relative myostatin (Mstn) gene expression in the normal non-cancerous small intestinal tissue (normal tissue) ( $\mathrm{n}=4)$ and polyps $(\mathrm{n}=3)$ of 17 to 19 week old $A p c^{\mathrm{Min} / \mathrm{t}}$ mice. Small intestine $(\mathrm{n}=3)$ and GAS muscles $(n=4)$ from 17 to 20 week old wild type mice were used as controls. Gene expression was normalized to Gapdh. Significance was assessed by one-way ANOVA followed by Tukey's post-hoc test: ${ }^{*} \mathrm{p}<0.05$, $* * \mathrm{p}<0.01, * * * \mathrm{p}<0.001 . \Delta \mathrm{Ct}$ was used to calculate significance.

Relative gene expression of B) interleukin-6 (Il6), C) TNF $\alpha(T N F)$, variant 1, and D) interferon gamma (Ifng), in the normal non-cancerous small intestinal tissue (normal tissue) $(\mathrm{n}=4)$ and polyps $(\mathrm{n}=3)$ of 17 to 19 week old $A p c^{M i n /+}$ mice. Small intestine (n=3) from 17 to 19 week old wild type mice were used as controls. Gene expression was normalized to Gapdh. Significance was assessed by one-way ANOVA followed by Tukey's post-hoc test: ${ }^{*} \mathrm{p}<0.05,{ }^{*} \mathrm{p}<0.01,{ }^{* * *} \mathrm{p}<0.001 . \Delta \mathrm{Ct}$ was used to calculate significance. For Figures 4.5.1A and 4.5.1B, $\mathrm{n}$ represents the number of mice used for this experiment. However, with respect to the data collected for the normal non-cancerous small intestinal tissue, wild type small intestine, and polyps, it must be noted that the small intestine was divided into 4 equal parts per mouse. Thus, each data point represents the fold change for a particular region of the small intestine. 

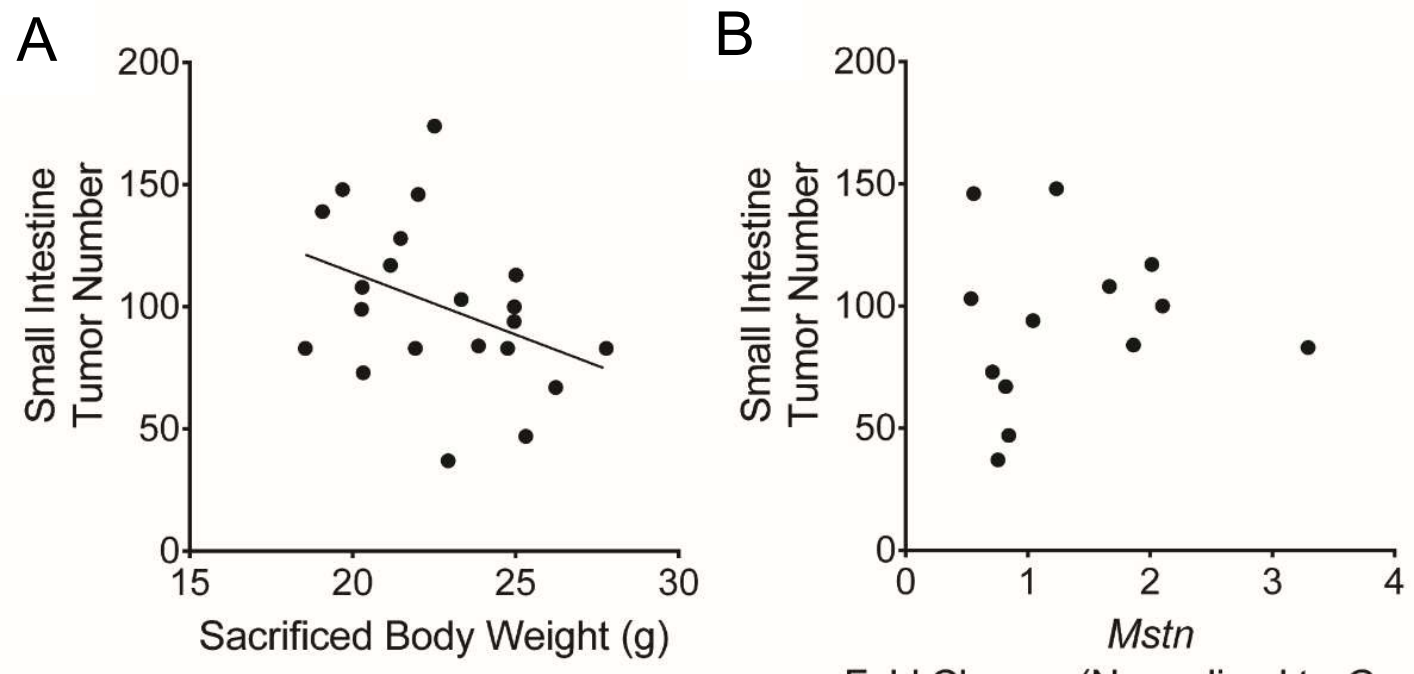

Fold Change (Normalized to Gapdh)

Figure 4.5.2: Small intestine polyp number did not correlate with male $A \mathrm{pc}^{\mathrm{Min} /+}$ body weight or myostatin gene expression.

A) The body weight (grams) of male $\mathrm{ApC}^{\mathrm{Min} / \mathrm{+}}$ mice against their corresponding small intestine tumor number $(n=21)$. Linear regression analysis was performed to determine whether the body weight was correlated with the small intestine tumor number. The mice were between 17 to 21 weeks old. Line-of-best fit is represented in the graph. $\mathrm{R}^{2}=0.1453 \mathrm{p}=0.0883$.

B) Relative gene expression of myostatin (Mstn) from the GAS muscles of $A p c^{\mathrm{Min} /+}$ mice against their corresponding small intestine tumor number $(\mathrm{n}=13)$. The mice were between 17 to 20 weeks old. Gene expression was normalized to Gapdh. 


\subsection{Soluble ActRIIB administration partially halted the loss of skeletal muscle mass and marginally improved grip strength in cachectic $\mathrm{Apc}^{\mathrm{Min} / \mathrm{+}}$ mice}

To test whether myostatin contributes to the pathogenesis of cancer-induced skeletal muscle wasting, we next inhibited the activity of myostatin by the administration of soluble ActRIIB (sActRIIB) to $A p c^{\mathrm{Min} /+}$ mice undergoing cachexia. We initially aimed to administer $5 \mu \mathrm{g} / \mathrm{gram}$ body weight of sActRIIB, three times per week, to 16 week old male $A p c^{\mathrm{Min} /+}$ mice for a period of 4 weeks (Figure 4.6.1). This dosage was chosen based on a previous study conducted by our laboratory [280]. However, 3 mice reacted adversely to this dosage, and unfortunately died, prompting us to reduce the concentration of the sActRIIB to $2.5 \mu \mathrm{g} / \mathrm{gram}$ body weight (Table 7.6.1). The sActRIIB dose was increased to $4.5 \mu \mathrm{g} / \mathrm{gram}$ body weight during the course of the experiment (Table 7.6.1). Despite this increase in SActRIIB dosage, we failed to observe any improvement in the growth and the percentage change in body weight of the sActRIIB treated $A p c^{\mathrm{Min} /+}$ mice when compared to the dialysis buffer treated controls (Figures 4.6.1A and 4.6.1B). Weekly food consumption was unaffected by the sActRIIB treatment (Figure 4.6.1C). Food consumption was measured by recording the difference between the food added at the start of the week and the food remaining at the end of the week for a cage housing a treatment group divided by the number of mice housed in the stated cage. At the end of the trial, sActRIIB treatment failed to increase the body weights of the cachectic $\mathrm{Apc}^{\mathrm{Min} /+}$ mice (Figure 4.6.1D).

Prior to the sacrifice of these animals, the forelimb grip strength was measured to determine whether the pharmacological inhibition of myostatin improved skeletal muscle strength and function (Figure 4.6.2A). In spite of the continuous decrease in body weight exhibited during the trial, we noted that the cachectic $A p c^{\mathrm{Min} /+}$ mice treated with sActRIIB displayed a significantly stronger grip strength than the dialysis buffer treated controls (Figure 4.6.2A). The grip strength of the sActRIIB treated $\mathrm{Apc}^{\mathrm{Min} / \mathrm{+}}$ mice were far weaker than the age matched wild type controls, indicating that sActRIIB treatment was incapable of restoring muscle function to that of wild type mice (Table 7.6.2). The increased grip strength observed in the sActRIIB treated animals corresponded to a larger TA, GAS, and QUAD mass (Figure 4.6.2B, Tables 7.6.3 and 7.6.4). However, this increase in both grip strength and muscle mass observed in the sActRIIB treated group did not yield a significant increase in the TA myofiber cross-sectional area (Figures 4.6.3 and 7.6.1). Adipose tissue weights and tibia lengths in the sActRIIB treated $A p c^{\mathrm{Min} /+}$ mice were 
similar to the dialysis buffer treated controls (Figure 4.6.2C and Table 7.6.5). It must be noted that three mice from the dialysis buffer treated group were omitted from our analysis because these mice failed to exhibit the symptoms of cachexia, such as reduced skeletal muscle weight and decreased adiposity (Figures 4.6.2A, 4.6.2B, Tables 7.6.3 and 7.6.4). 

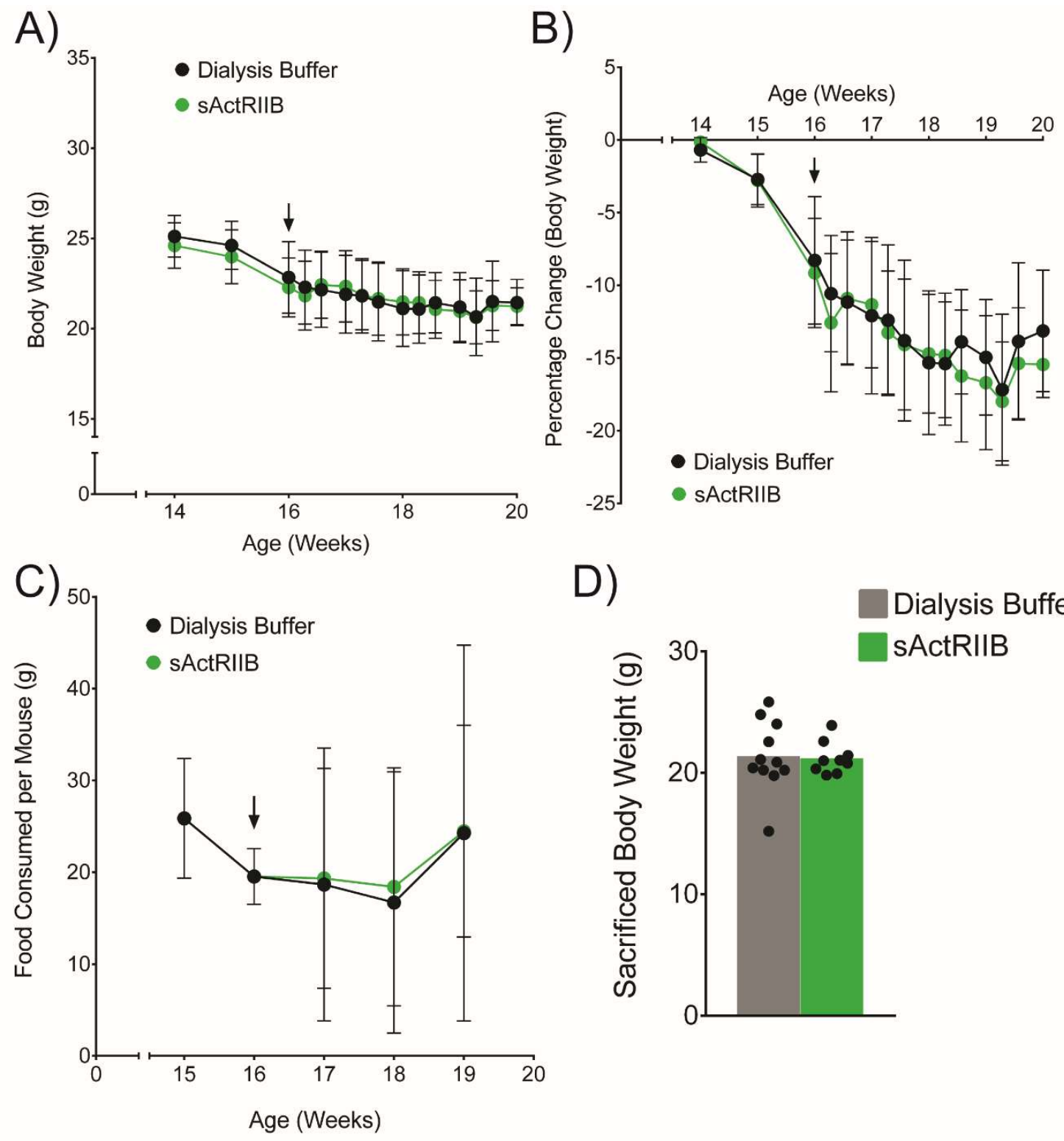

D)
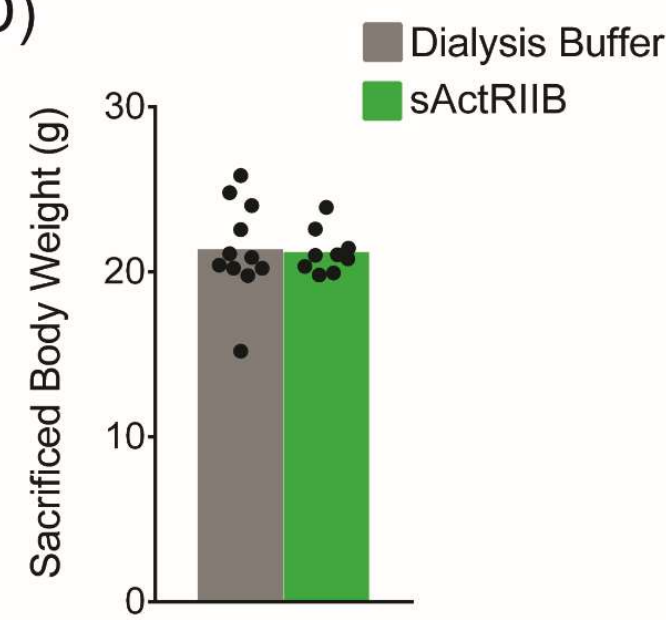

Figure 4.6.1: The loss of body mass due to cancer cachexia in male $\mathrm{Apc}^{\mathrm{Min}+}$ mice was not prevented by sActRIIB treatment.

A) Body weights (grams) and B) Percentage change in body weight (\%) for dialysis buffer $(\mathrm{n}=8-11)$ and sActRIIB treated $A p c^{\mathrm{Min} /+}(\mathrm{n}=9-12)$ mice. Treatment date (16 weeks) is indicated by an arrow. Error bars denote $\pm 95 \%$ CI. Three mice perished during the course of the sActRIIB treatment. Live body weights were not measured for three dialysis buffer treated mice on the last day of the treatment regime.

C) Food consumed per mouse (grams) for dialysis buffer $(\mathrm{n}=3)$ and sActRIIB treated $A p c^{\operatorname{Min} /+}(\mathrm{n}=3)$ mice, where $\mathrm{n}$ represents the number of cages. Treatment date (16 weeks) is indicated by an arrow. Food consumption was measured from 14 weeks. Error bars denote $\pm 95 \%$ CI.

D) Sacrificed body weights (grams) for dialysis buffer ( $\mathrm{n}=11)$ and sActRIIB treated $A p c^{\operatorname{Min} /+}(\mathrm{n}=9)$ mice. Significance was assessed by Student's t-test with Welch correction. No significant difference was observed. 

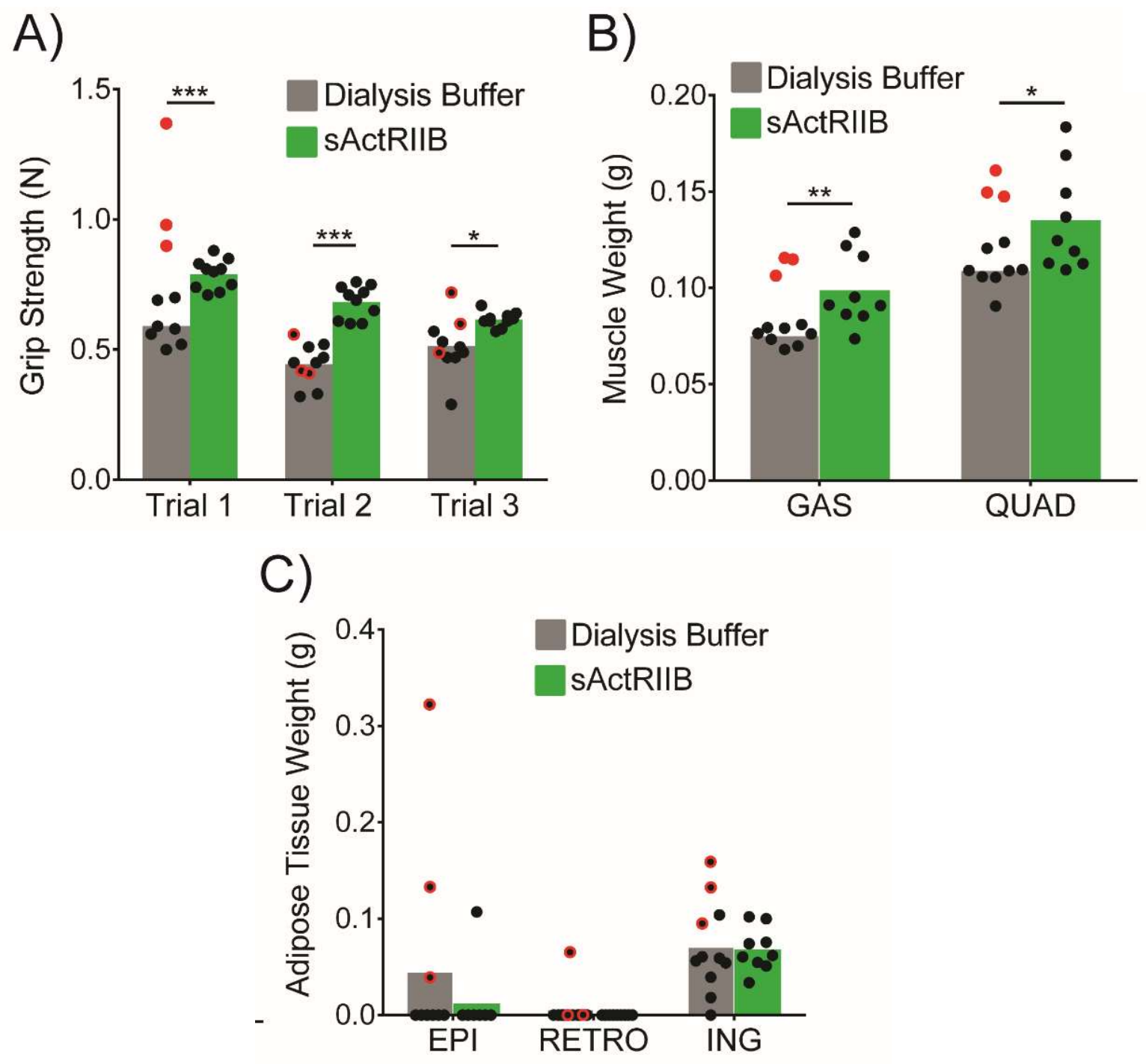

Figure 4.6.2: Soluble ActRIIB treatment of male $A p c^{M i n /+}$ mice at 16 weeks partially prevented skeletal muscle atrophy and improved forelimb grip strength.

A) Forelimb grip strength measured in Newtons (N) for dialysis buffer $(\mathrm{n}=11)$ and sActRIIB treated $\mathrm{Ap} \mathrm{c}^{\mathrm{Min} /+}$ $(n=9)$ mice. The grip strengths of three mice that are omitted from the analysis of trial 1 are highlighted as red dots. The same mice that were omitted in trial 1 but not omitted from trial 2 and 3 are highlighted as black dots with a red outline. Significance was assessed by Student's t-test with Welch correction: ${ }^{*} p<0.05$, $* * p<0.01, * * * p<0.001$.

B) Gastrocnemius (GAS) and quadriceps (QUAD) weights (grams) for dialysis buffer ( $\mathrm{n}=11$ ) and sActRIIB treated $\mathrm{Apc}^{\mathrm{Min} /+}(\mathrm{n}=9)$ mice. Three mice that were omitted from the analysis are highlighted as red dots. Significance was assessed by Student's t-test with Welch correction: ${ }^{*} p<0.05,{ }^{* *} p<0.01,{ }^{* * *} p<0.001$.

C) Epididymal (EPI), retroperitoneal (RETRO), and inguinal (ING) adipose tissue weights (grams) for dialysis buffer $(\mathrm{n}=11)$ and sActRIIB treated $A p c^{M i n /+}(\mathrm{n}=9)$ mice. Mice were not omitted from this analysis. However, the three mice that were omitted from the previous analysis are highlighted as black dots with a red outline. Significance was assessed by Student's t-test with Welch correction: No significant difference was observed. 


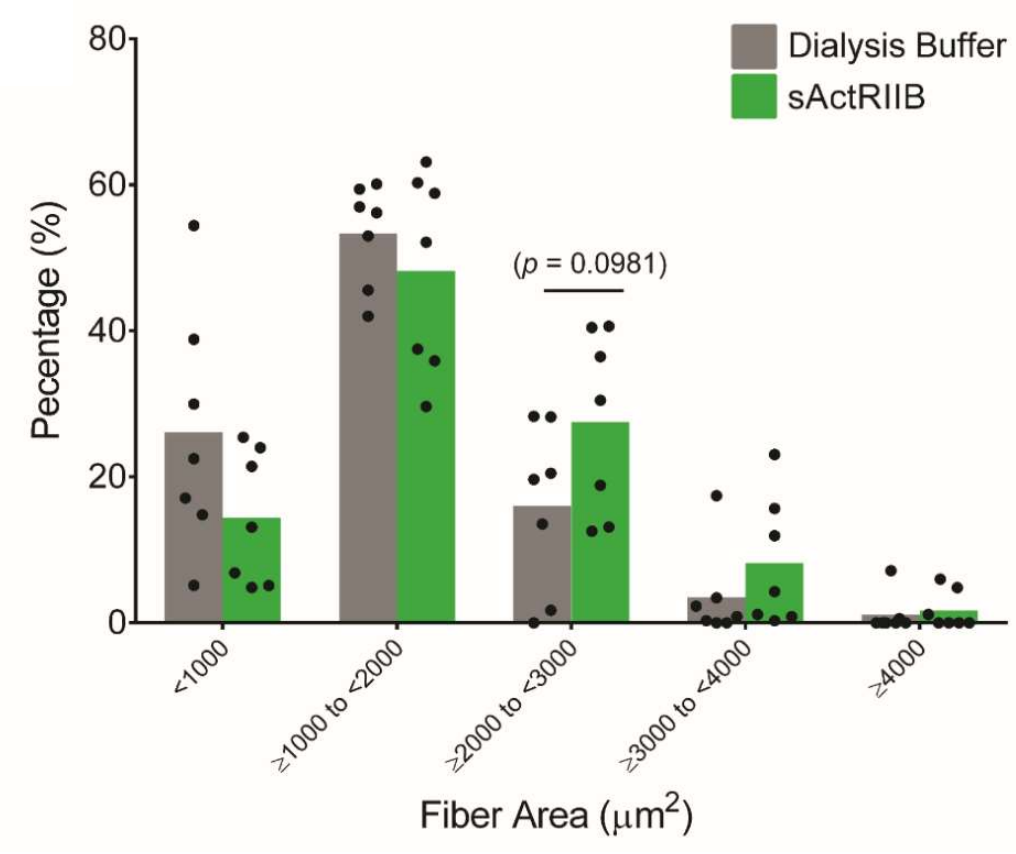

Figure 4.6.3: Soluble ActRIIB treatment of male $\mathrm{Apc}^{\mathrm{Min} /+}$ mice at 16 weeks failed to improve the TA myofiber cross-sectional area.

Percentage frequency distribution for the TA myofiber cross-sectional area $\left(\mu \mathrm{m}^{2}\right)$ from sActRIIB $(n=7)$ and dialysis buffer ( $\mathrm{n}=7)$ treated $A p c^{\mathrm{Min} /+}$ mice (left). Significance was assessed by Student's t-test with Welch correction: ${ }^{*} p<0.05,{ }^{* *} p<0.01,{ }^{* * *} p<0.001$. Representative H\&E images of cryosectioned TA are found in Figure 7.6.1. 
To determine whether the administration of sActRIIB could prevent the onset of cancer cachexia in the $\mathrm{Apc}^{\mathrm{Min} /+}$ mouse, we administered sActRIIB to pre-cachectic 14 week old $A p c^{\mathrm{Min} /+}$ mice (see Table 7.6.6 for information on the dosage). The administration of sActRIIB to these mice failed to halt the occurrence of cancer cachexia in the $\mathrm{Apc}^{\mathrm{Min} /+}$ mice (Figure 4.6.4). Specifically, the body weights of the sActRIIB treated animals decreased at the same rate as the dialysis buffer treated controls (Figures 4.6.4A and 4.6.4B). In addition, the sacrificed body weight of sActRIIB treated $\mathrm{ApC}^{\mathrm{Min} /+}$ mice measured at the end of the trial were no different to the mice that received dialysis buffer (Figure 4.6.4C). On the other hand, the forelimb grip strength of the sActRIIB treated mice were far stronger than the dialysis buffer treated controls (Figure 4.6.5A). Unlike the previous trial, however, we were unable to observe a marked increase in the skeletal muscle size of sActRIIB treated animals (Figure 4.6.5B and Table 7.6.7). The administration of sActRIIB did not alter adipose tissue weights and tibia lengths (Figure 4.6.5C and Table 7.6.8). 


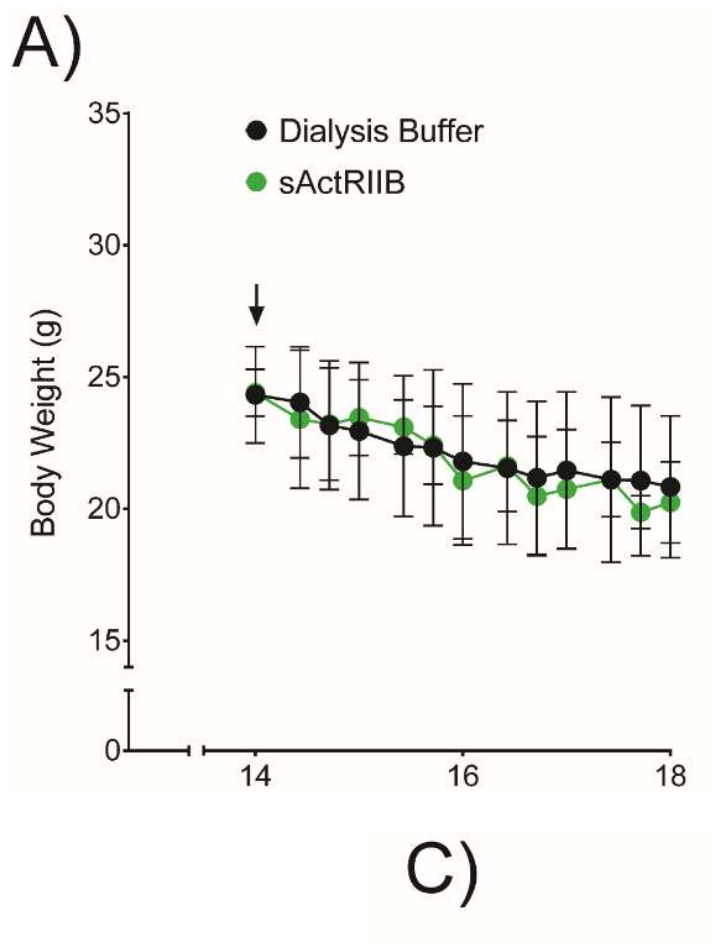

\section{B)}

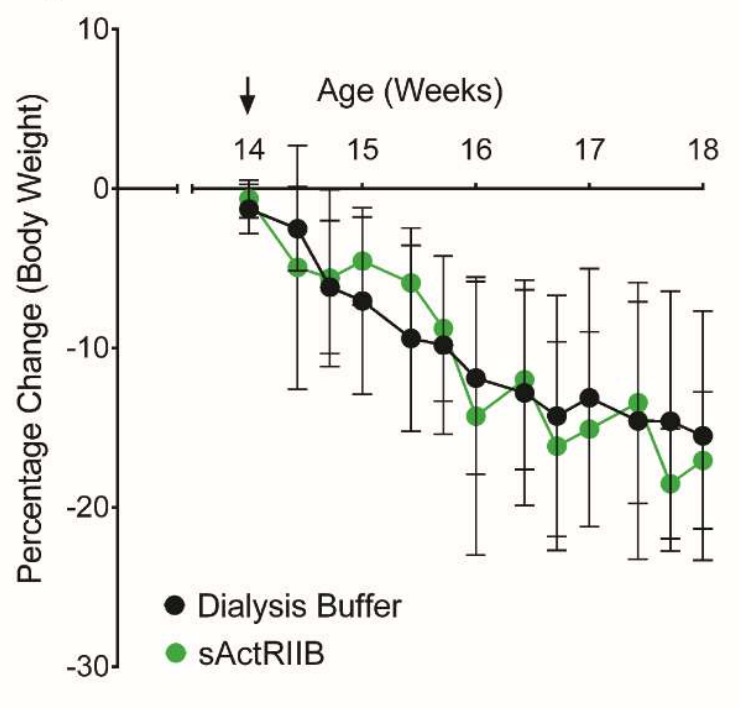

Dialysis Buffer

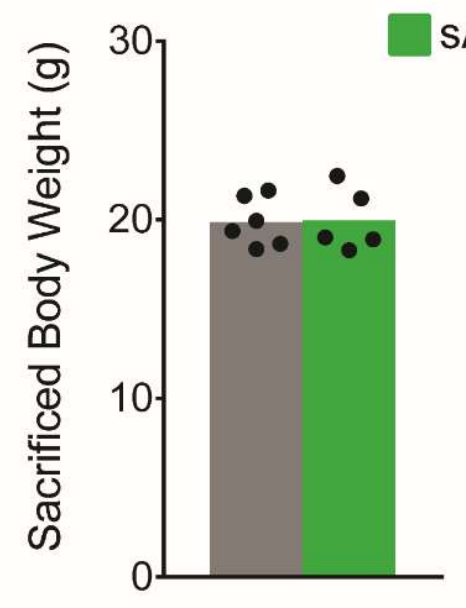

Figure 4.6.4: Soluble ActRIIB treatment of pre-cachectic male $A p c^{\mathrm{Min} /+}$ mice failed to prevent the onset of cancer cachexia.

A) Body weights (grams) and B) Percentage change in body weight (\%) for dialysis buffer ( $\mathrm{n}=7)$ and sActRIIB treated $A p c^{\mathrm{Min} /+}$ ( $\left.\mathrm{n}=5-6\right)$ mice. Treatment date (14 weeks) is indicated by an arrow. One mouse in the sActRIIB treatment group died during the course of the experiment. Error bars denote \pm $95 \%$ CI.

C) Sacrificed body weights (grams) for dialysis buffer $(\mathrm{n}=6)$ and sActRIIB treated $\operatorname{Apc}^{\mathrm{Min} /+}(\mathrm{n}=5)$ mice. One mouse from the dialysis buffer treated group, mouse ID 650, was not dissected, as it was surmised to be non-cachectic. Significance was assessed by Student's t-test with Welch correction: No significant difference was observed. 


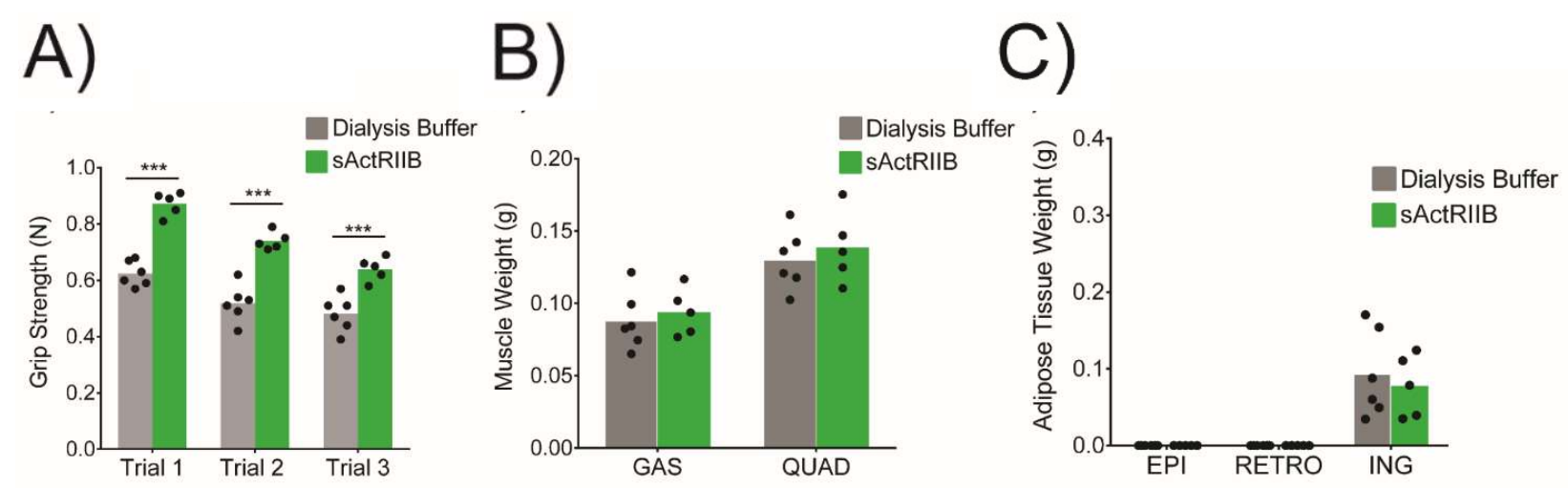

Figure 4.6.5: Soluble ActRIIB treatment of pre-cachectic male $\mathrm{Apc}^{\mathrm{Min} / \mathrm{+}}$ mice partially improved forelimb grip strength.

A) Forelimb grip strength measured in Newtons $(\mathrm{N})$ for dialysis buffer $(\mathrm{n}=6)$ and sActRIIB treated $A p c^{\operatorname{Min} /+}(\mathrm{n}=5)$ mice. One mouse from the dialysis buffer treated group, mouse ID 650, was not measured for forelimb grip strength, as it was surmised to be non-cachectic. Significance was assessed by Student's t-test with Welch correction: ${ }^{*} p<0.05,{ }^{* *} p<0.01, * * * p<0.001$.

B) Gastrocnemius (GAS) and quadriceps (QUAD) weights (grams) for dialysis buffer $(n=6)$ and sActRIIB treated $\mathrm{Apc}^{\mathrm{Min} /+}(\mathrm{n}=5)$ mice. One mouse from the dialysis buffer treated group, mouse ID 650 , was not dissected, as it was surmised to be non-cachectic. Significance was assessed by Student's t-test with Welch correction: No significant difference was observed.

C) Epididymal (EPI), retroperitoneal (RETRO), and inguinal (ING) adipose tissue weights (grams) for dialysis buffer $(\mathrm{n}=6)$ and sActRIIB treated $\mathrm{Apc}^{\mathrm{Min} /+}(\mathrm{n}=5)$ mice. One mouse from the dialysis buffer treated group, mouse ID 650, was not dissected, as it was surmised to be non-cachectic. Significance was assessed by Student's t-test with Welch correction: No significant difference was observed. 
We next examined whether the administration of sActRIIB influenced the intestinal tumor biology of the $\mathrm{Apc}^{\mathrm{Min} /+}$ mouse. To investigate this question, we measured the small intestine tumor number of the same $A p c^{\mathrm{Min} /+}$ mice treated with sActRIIB at 16 weeks of age (see Figures 4.6.1-4.6.3). According to Figure 4.6.4, the small intestinal tumor number and the average tumor diameter garnered from the sActRIIB treated animals were similar to the dialysis buffer treated controls (Figures 4.6.6A and 4.6.6C). However, tumors with a diameter smaller than $1 \mathrm{~mm}$ were significantly greater in the dialysis buffer treated controls compared to sActRIIB treated $\mathrm{Ap} \mathrm{c}^{\mathrm{Min} /+}$ mice (Figure 4.6.6B). This difference was not recapitulated when we represented the tumor diameter as a percentage of the total number of tumors per mouse (Figure 4.6.6D). 


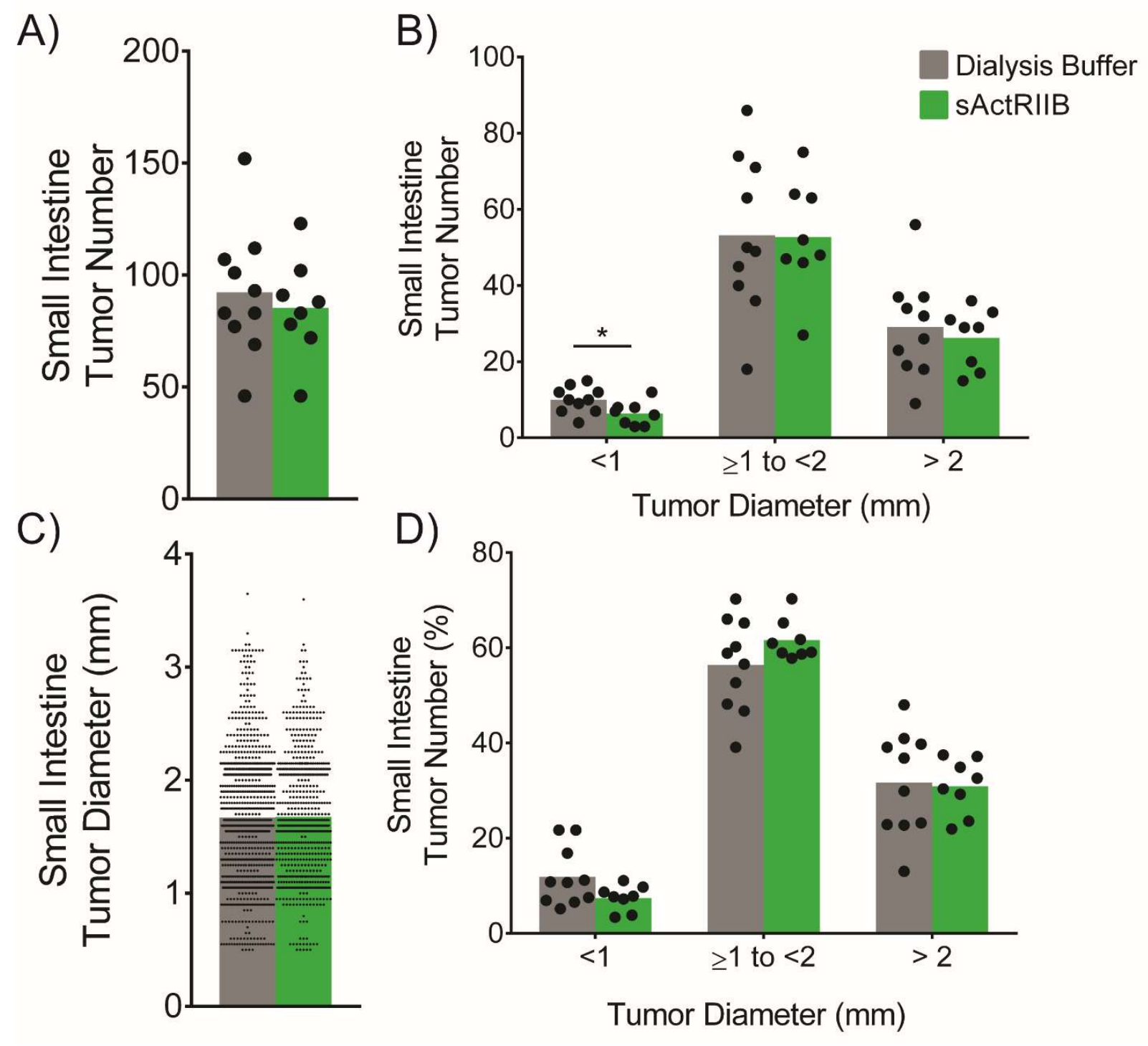

Figure 4.6.6: The small intestine tumor biology of $A p c^{M i n /+}$ mice administered with sActRIIB from 16 weeks of age.

A) Small intestine tumor number for dialysis buffer $(\mathrm{n}=10)$ and sActRIIB treated $A p c^{\operatorname{Min} /+}(\mathrm{n}=8)$ mice. Significance was assessed by Student's t-test with Welch correction: No significant difference was observed.

B) Small intestine tumor diameter $(\mathrm{mm})$ for dialysis buffer $(\mathrm{n}=10)$ and sActRIIB treated $\mathrm{Apc}^{\mathrm{Min} /+}(\mathrm{n}=8)$ mice. Significance was assessed by Student's t-test with Welch correction: ${ }^{*} p<0.05, * * p<0.01$, $* * * p<0.001$.

C) Average small intestine tumor diameter $(\mathrm{mm})$ for dialysis buffer $(\mathrm{n}=10)$ and sActRIIB treated $\operatorname{Apc}^{\mathrm{Min} /+}(\mathrm{n}=8)$ mice. Please note that each data point represents the diameter of one tumor. Significance was assessed by Student's t-test with Welch correction: No significant difference was observed.

D) Small intestine tumor diameter (\%)for dialysis buffer (n=10) and sActRIIB treated $\operatorname{Apc}^{\mathrm{Min} /+}(\mathrm{n}=8)$ mice. Each data point represents the number of tumors that belong to the stated diameter over the total number of tumors per animal. Data was represented as a percentage. Significance was assessed by Student's t-test with Welch correction: No significant difference was observed. 


\subsection{The genetic ablation of myostatin prevented the onset of cancer cachexia in $\mathrm{Apc}^{\mathrm{Min} /+}$ mice}

The pharmacological inactivation of myostatin through sActRIIB resulted in the slight rescue of skeletal muscle atrophy in cachectic $\mathrm{Apc}^{\mathrm{Min} /+}$ mice. To further delineate the involvement of myostatin in promoting cancer cachexia, we next studied the phenotype of male $A p c^{\operatorname{Min} /+}$ mice in a myostatin hemizygote $\left(M_{\left.s t n^{+/-}\right)}\right.$and nullizygote $\left(\mathrm{Msth}^{-/}\right)$. The $\mathrm{Mstn}^{+/}: \because A p c^{\mathrm{Min} /+}$ mice were generated by crossing $\mathrm{Mstn}^{+/+}:: \mathrm{Apc}^{\mathrm{Min} /+}$ male mice to female $\mathrm{Mstn}^{-/}$mice. $\mathrm{Mstn}^{-/}: \because \mathrm{Apc}^{\mathrm{Min} /+}$ mice were generated by the $\mathrm{Mstn}^{+/}: \because A p c^{\mathrm{Min} /+}$ male and $\mathrm{Mstn}^{+/}$female breeding scheme. Wild type $A p c^{M i n /+}$ mice $\left(\mathrm{Mstn}^{+/+}: \because A p c^{M i n /+}\right)$ mice were maintained as a separate line. Similar to the $A p c^{M i n /+}$ mice, $M_{s t n}{ }^{-/}$mice in our colony are C57BL/6.

Preliminary evidence for the relative resistance of $\mathrm{Mstn}^{+/-} \because: A p c^{\mathrm{Min} /+}$ and $\mathrm{Msth}^{-/} \because: A p c^{\mathrm{Min} /+}$ mice to cancer cachexia came from the examination of their growth curve (Figure 4.7.1A). Both $\mathrm{Mstn}^{+/-}$ $\because A p c^{M i n /+}$ and $\mathrm{Mstn}^{-1-}: \because A p c^{\mathrm{Min} /+}$ male mice failed to exhibit a precipitous reduction in their body weight between 14 to 18 weeks of age (Figure 4.7.1A). To confirm whether $A p c^{M i n /+}$ mice on the myostatin hemizygote and nullizygote background displayed the hallmarks of cancer cachexia, we terminated $\mathrm{Mstn}^{+/-}: \because A p c^{\mathrm{Min} /+}$ and $\mathrm{Mstn}^{-/} \because: A p \mathrm{Cin}^{\mathrm{Mi+}}$ male mice as well as their respective controls at 18 weeks and compared their body weight and skeletal muscle weights to 18 week old $\mathrm{Mstn}^{+/+}:: A p c^{\mathrm{Min/+}}$ and $\mathrm{Mstn}^{+/+}: \because A p c^{+/+}$mice (Figures 4.7.1B and 4.7.1C). 18 weeks of age was chosen as most $\mathrm{Mstn}^{+/+}:: A p \mathrm{cMin/+}^{\mathrm{m}}$ male mice exhibit the symptoms of cancer cachexia.

As expected, 18 week old $\mathrm{Mstn}^{+/+}:: A p \mathrm{c}^{\mathrm{Min} /+}$ mice demonstrated a marked reduction in their body weight and their skeletal muscle mass when compared to their wild type, $\mathrm{Mstn}^{+/+}:: A p c^{+/+}$controls (Figures 4.7.1B, 4.7.1C, and Table 7.7.1). Conversely, $\mathrm{Mstn}^{-/} \because: A p c^{\mathrm{Min} /+}$ and $\mathrm{Msth}^{-/} \because: \mathrm{Apc}^{+/+}$mice displayed a similar body weight and skeletal muscle mass (Figures 4.7.1B, 4.7.1C, and Table 7.7.2). The $\mathrm{Mstn}^{-/}: \because A p \mathrm{CMin/+}^{\mathrm{M}}$ body weights and skeletal muscle weights were significantly larger than the $\mathrm{Mstn}^{+/+}: \because A p c^{\mathrm{Min} /+}$ and $\mathrm{Mstn}^{+/-}: \because A p c^{\mathrm{Min} /+}$ mice, indicating that the absence of myostatin may preserve body weight and skeletal muscle mass despite the onset of intestinal tumors (Figures 4.7.1B and 4.7.1C). It was interesting to note that $\mathrm{Mstn}^{+/} \because \because A p \mathrm{c}^{\mathrm{Min} /+}$ mice were smaller in their body weight, GAS, and QUAD weights compared to their respective wild type controls (Figures 4.7.1B, 4.7.1C, and Table 7.7.2). 


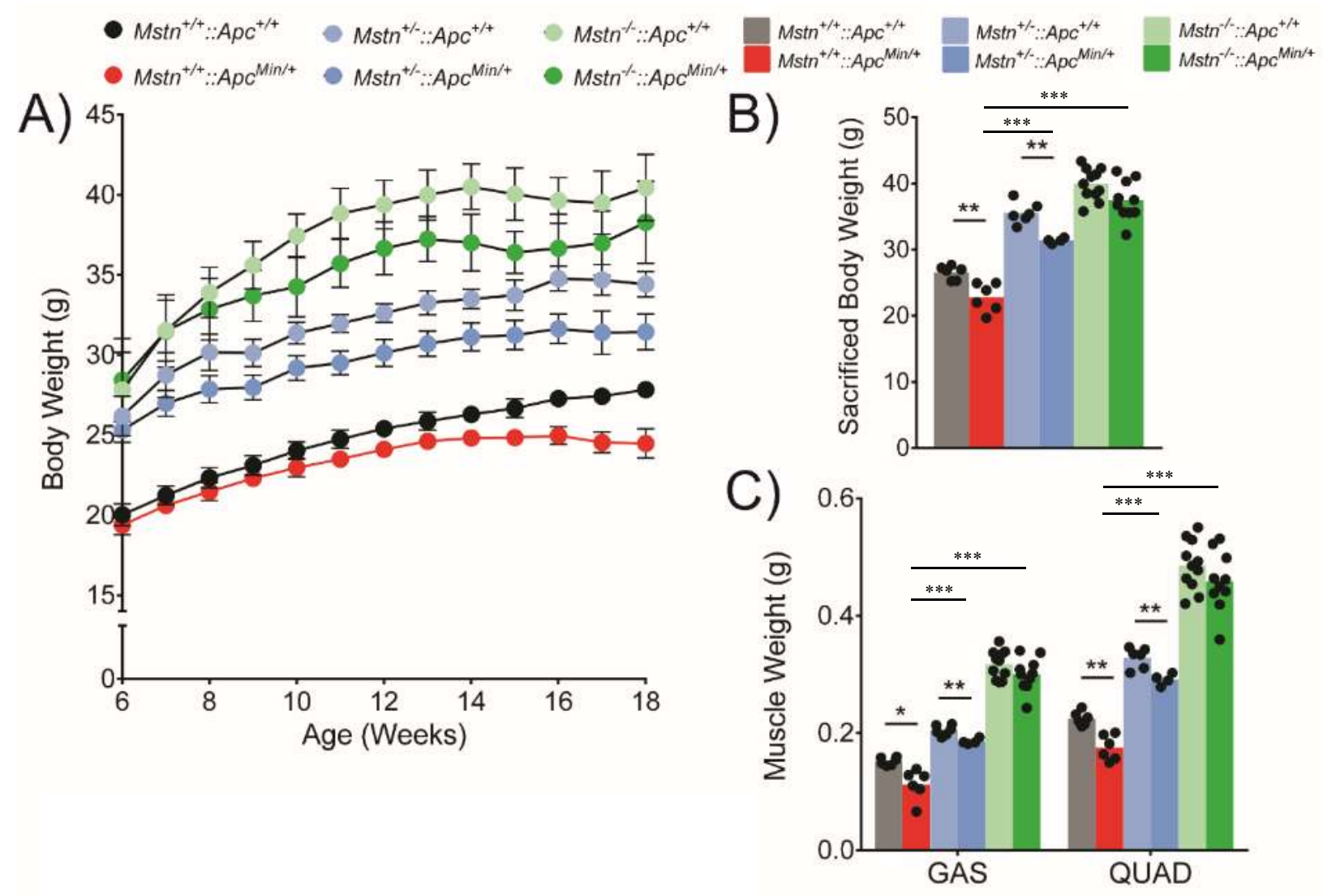

Figure 4.7.1: Genetic ablation of myostatin prevented the onset of cancer cachexia in 18 week old $A p c^{M i n /+}$ mice.

A) Weekly body weights for male $M_{s t n}{ }^{+/+}: \because A p c^{M i n /+}(\mathrm{n}=9-50), M_{s t n}{ }^{+/} \because: A p c^{M i n /+}(\mathrm{n}=10-20)$, and Mstn ${ }_{1} \because A p c^{M i n /+}(\mathrm{n}=7-22)$ mice and their respective wild type controls, $\operatorname{Mstn}^{+/+}: \because A p c^{+/+}(\mathrm{n}=11-38), M_{s t n}{ }^{+/}$ $\therefore A p c^{+/+}(\mathrm{n}=7-17)$, and $M s t^{-/}: \because A p c^{+/+}(\mathrm{n}=8-20)$ mice. Body weights are presented from 6 to 18 weeks. Data for $\mathrm{Msth}^{+/+}: \because A p c^{\mathrm{Min} /+}$ and $\mathrm{Mstn}^{+/+}: \because A p c^{+/+}$mice were acquired from Figure 4.1.1. Error bars denote $\pm 95 \%$ CI.

B) Sacrificed body weights (grams) for $M_{s t n}{ }^{+/+}: \because A p c^{M i n /+}(\mathrm{n}=6), M s t n^{+/}: \because A p c^{M i n /+}(\mathrm{n}=4)$, and $\mathrm{Mstn}^{-/ /}$ $\because A p c^{M i n /+}(\mathrm{n}=10)$ mice and their respective wild type controls, $\operatorname{Mstn}^{+/+}: \because A p c^{+/+}(\mathrm{n}=6), M_{s t n^{+/}}:: A p c^{+/+}$ $(\mathrm{n}=6)$, and $M \operatorname{stn}^{-/}: \because A p c^{+/+}(\mathrm{n}=11)$ mice. Significance between $A p c^{M i n /+}$ and $A p c^{+/+}$was assessed by Student's t-test with Welch correction. Significance between $\mathrm{Mstn}^{+/+}: \because A p c^{M i n /+}, M_{s t n}{ }^{+/}: \because A p c^{M i n /+}$, and $M s t n^{-/}: \because A p c^{M i n /+}$ was assessed by one-way ANOVA followed by Tukey's post-hoc test: ${ }^{*} p<0.05$, $* * p<0.01, * * * p<0.001$.

C) Gastrocnemius (GAS) and quadriceps (QUAD) weights (grams) for $\mathrm{Mstn}^{+/+}:: A p c^{\mathrm{Min} /+}(\mathrm{n}=6)$, $M_{s t n}{ }^{+/} \because: A p c^{M i n /+}(\mathrm{n}=4)$, and $M_{s t n^{-1}} \because A p c^{M i n /+}(\mathrm{n}=10)$ mice and their respective wild type controls, $\operatorname{Mstn}^{+/+}: \because A p c^{+/+}(\mathrm{n}=6), M_{s t n}^{+/} \because: A p c^{+/+}(\mathrm{n}=6)$, and $\operatorname{Mstn}^{-/}: \because A p c^{+/+}(\mathrm{n}=11)$ mice. Significance between $\mathrm{Apc}^{\mathrm{Min} /+}$ and $\mathrm{Apc}^{+/+}$was assessed by Student's t-test with Welch correction. Significance between $\mathrm{Mstn}^{+/+}: \because \mathrm{Apc}^{\mathrm{Min/+}}, \mathrm{Mstn}^{+/} \because \because A p c^{\mathrm{Min} /+}$, and $\mathrm{Mstn}^{-/-}: \because \mathrm{Apc}^{\mathrm{Min} /+}$ was assessed by one-way ANOVA followed by Tukey's post-hoc test: ${ }^{*} p<0.05,{ }^{* *} p<0.01,{ }^{* * *} p<0.001$. 


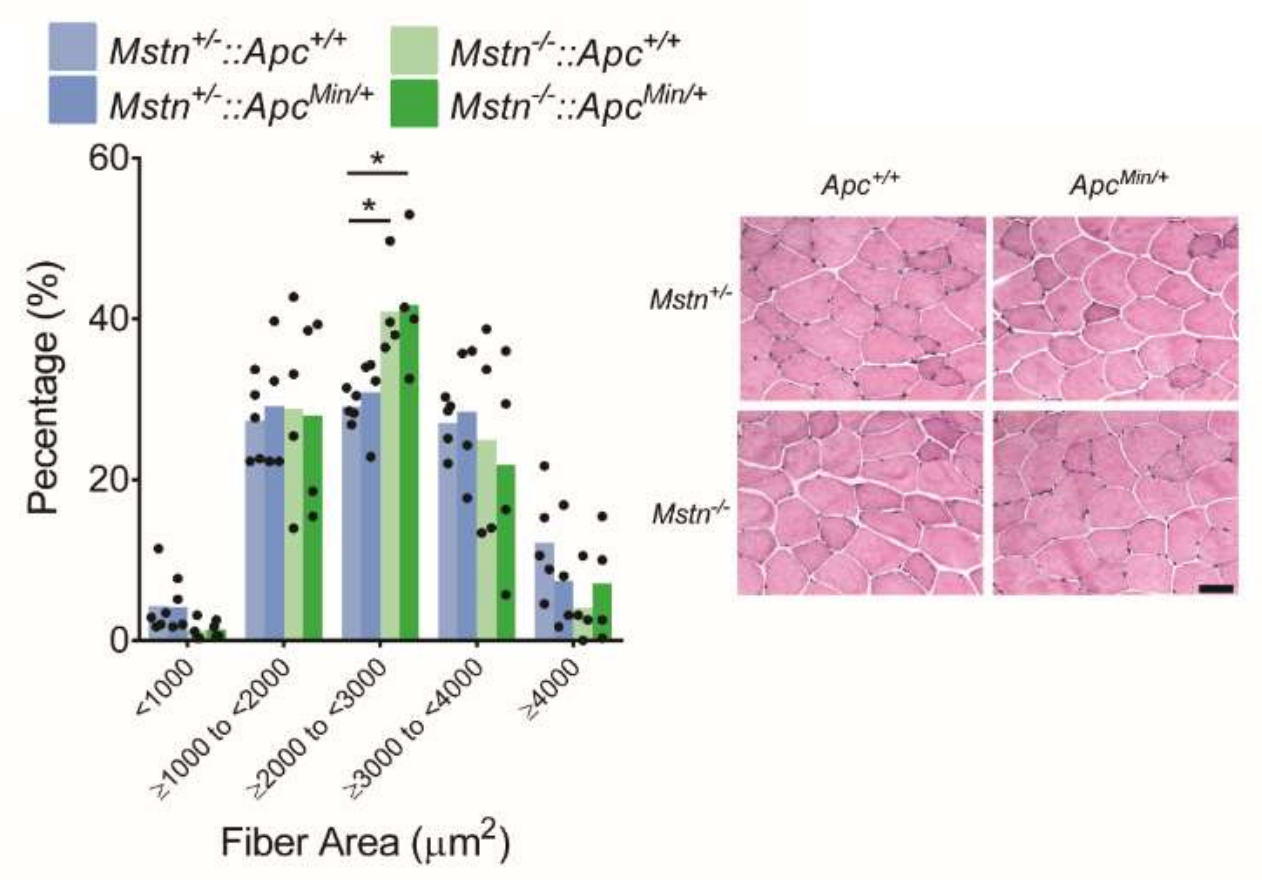

Figure 4.7.2: TA Myofiber cross-sectional area of 18 week old $\mathrm{Mstn}^{+/}: \because \mathrm{Apc}^{\mathrm{Min} /+}$ and $\mathrm{Mstn}^{-/-}$ $\because A p c^{M i n / 4}$ mice.

A) TA myofiber cross-sectional area $\left(\mu \mathrm{m}^{2}\right)$ for $\operatorname{Msth}^{+/} \because \because A p c^{\operatorname{Min} /+}(\mathrm{n}=4)$ and $\operatorname{Mstn}^{-/} \because: \operatorname{Apc}^{\operatorname{Min} /+}(\mathrm{n}=4)$ mice, and their respective wild type controls, $\mathrm{Mstn}^{+/} \because: \mathrm{Apc}^{+/+}(\mathrm{n}=5)$ and $\mathrm{Msth}^{-/} \because \because \mathrm{Apc}^{+/+}(\mathrm{n}=4)$ mice (Right). Each data point represents the number of myofibers that belong to the stated diameter over the total number of fibers counted per animal. Data is represented as a percentage Significance was assessed by one-way ANOVA followed by Tukey's post-hoc test: ${ }^{*} p<0.05,{ }^{*} p<0.01,{ }^{*} * * p<0.001$.

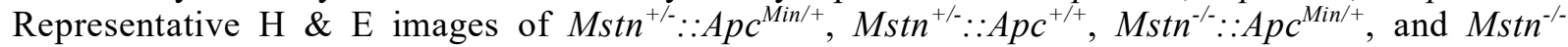
$\because A p c^{+/+}$cryosectioned TA. Scale bar represents $50 \mu \mathrm{m}($ Left $)$. 
The observed decrease in the body weight and muscle weight of 18 week old $\mathrm{Mstn}^{+-}: \because A p \mathrm{Ain}^{\mathrm{Mi}+}$ mice when compared to its respective control $\left(\mathrm{Mstn}^{+/}: \because \mathrm{Apc}^{+/+}\right)$was not due to the onset of cancer cachexia. In fact, the TA myofiber cross-sectional area of the $\mathrm{Mstn}^{+/}: \because A p \mathrm{C}^{\mathrm{Min} /+}$ mice was similar to the $\mathrm{Mstn}^{+/}: \because A p c^{+/+}$mice (Figure 4.7.2). More important, the protein expression of Atrogin-1 and MuRF1 in the GAS muscles of the $\mathrm{Mstn}^{+/}: \because A p \mathrm{AMin} /+^{\mathrm{m}}$ mice were comparable to the $\mathrm{Mstn}^{+/-}$ $\because A p c^{+/+}$controls (Figures 4.7.3A, 4.7.3B, and Figures 7.7.1-7.7.4). Likewise, the TA myofiber cross-sectional area and the protein amounts of Atrogin-1 and MuRF1 from 18 week old Mstr-/$\because A p c^{\mathrm{Min} /+}$ mice were also similar to their respective wild type controls (Figures 4.7.2, 4.7.3C, 4.7.3D, and Figures 7.7.1-7.7.4). The absence of cancer cachexia in the $\mathrm{Mstn}^{+/} \because: \mathrm{Apc}^{\mathrm{Min} /+}$ and $\mathrm{Mstn}^{-}$ ${ }^{\prime} \because A A p c^{\mathrm{Min} /+}$ mice was also apparent as the protein expression of Atrogin-1 and MuRF1 were significantly lower in these mice when compared to 18 week old $\mathrm{Mstn}^{+/+}: \because \mathrm{Apc}^{\mathrm{Min} /+}$ mice (Figures 4.7.3E, 4.7.3F, and Figures 7.7.1-7.7.4). Unlike the 18 week old $\mathrm{Mstn}^{+/+}:: A p c^{\mathrm{Min} /+}$ mice, both the $\mathrm{Mstn}^{+/}: \because \mathrm{Apc}^{\mathrm{Min} /+}$ and $\mathrm{Msth}^{-/} \because: \mathrm{Apc}^{\mathrm{Min} /+}$ mice exhibited a relative preservation in the adipose tissue mass, further confirming that the absence of myostatin in the $\mathrm{Apc}^{\mathrm{Min} /+}$ mice was sufficient to prevent cancer cachexia (Figure 4.7.4A). Furthermore, the spleen weights of the 18 week old $\mathrm{Mstn}^{+/+}: \because A p c^{\mathrm{Min} /+}$ mice were significantly larger than their respective wild type controls (Figure 4.7.4). An enlarged spleen, or splenomegaly, is indicative of anemia caused by the loss of blood through the intestinal tumors $[279,288,289]$. Splenomegaly was not recorded in the Mstn ${ }^{+/}$ $\because A p c^{\mathrm{Min} /+}$ and $\mathrm{Mstn}^{-/}: \because A p \mathrm{CMin/+}^{\mathrm{M}}$ mice when compared to their respective $\mathrm{Apc}^{+/+}$controls, suggesting the absence of anemia and possibly a lack of intestinal tumors in mice devoid of myostatin (Figure 4.7.4B). Additionally, tibia lengths of both $\mathrm{Mstn}^{+/} \because \because \mathrm{Apc}^{\mathrm{Min} /+}$ and $\mathrm{Mstn}^{-/}$ $\because A p c^{M i n /+}$ mice were larger than their respective $M_{s t n}^{+/+}$controls (Table 7.7.2). Mstn ${ }^{-/} \because A p c^{+/+}$ mice also demonstrated a marked increase in tibia length compared to $\mathrm{Mstn}^{+/+}: \because \mathrm{Apc}^{\mathrm{Min} /+}$ mice (Table 7.7.2). Furthermore, $M s t n^{+/+}:: A p c^{M i n /+}$ tibia lengths were significantly smaller than the $M_{s t n^{+/+}}: A p c^{+/+}$controls (Table 7.7.2). 


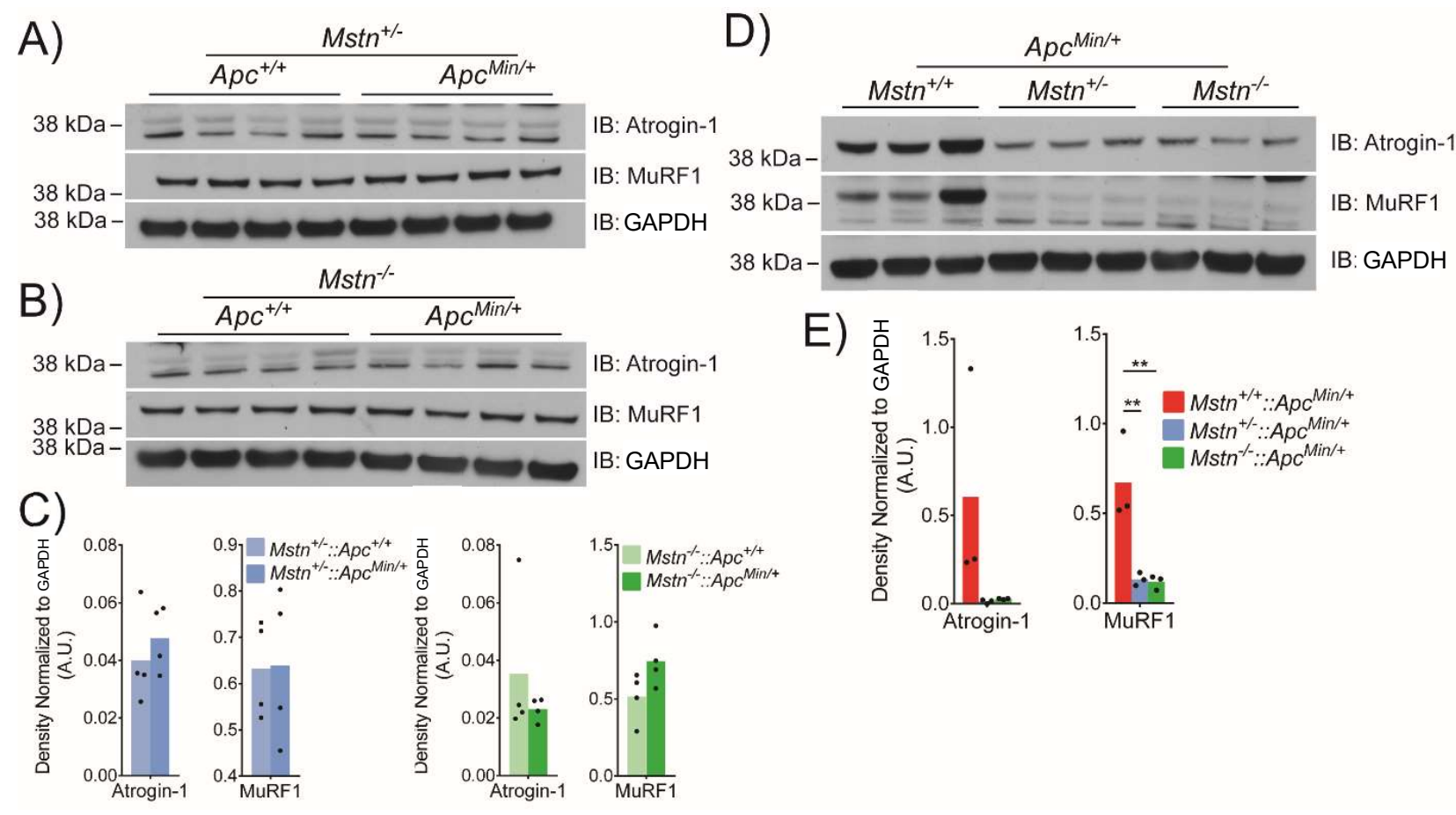

Figure 4.7.3: The absence of myostatin suppressed the over-expression of the ubiquitin proteasome pathway in skeletal muscles of 18 week old $\mathrm{Mstn}^{+-}:: \mathrm{Apc}^{\mathrm{Min} /+}$ and $\mathrm{Mstn}^{-/-}:: \mathrm{Apc}^{\mathrm{Min} /+}$ mice.

A) Immunoblots for Atrogin- 1 and MuRF1 for the GAS muscles isolated from $M \operatorname{stn}^{+/}: \because A p c^{\operatorname{Min} /+}(\mathrm{n}=4)$ and $\mathrm{Mstn}^{+/}: \because \mathrm{Apc}^{+/+}(\mathrm{n}=4)$ mice. GAPDH was used as a loading control. GAPDH shown here corresponds to the loading control used for MuRF1 (Figure 7.7.4A). The molecular weight markers are represented on the left side of the immunoblots. Immunoblots used for this figure and their corresponding GAPDH loading controls are displayed in Figures 7.7.1-7.7.4.

B) Immunoblots for Atrogin-1 and MuRF1 for the GAS muscles isolated from $\operatorname{Mstn}^{-1}: \because A p c^{M i n /+}(\mathrm{n}=4)$ and $M s t n^{-/}: \because \mathrm{Apc}^{+/+}(\mathrm{n}=4)$ mice. GAPDH was used as a loading control. GAPDH shown here corresponds to the loading control used for MuRF1 (Figure 7.7.4B). The molecular weight markers are represented on the left side of the immunoblots. Immunoblots used for this figure and their corresponding GAPDH loading controls are displayed in Figures 7.7.1-7.7.4.

C) Densitometry for Atrogin-1 and MuRF1 normalized to their corresponding GAPDH loading controls for immunoblots represented in Figures 4.7.3A and 4.7.3B. Significance was assessed by Student's t-test with Welch correction: No significant difference was observed.

D) Immunoblots for Atrogin-1 and MuRF1 for the GAS muscles isolated from $\operatorname{Mstn}^{+/+}: \because A p c^{\operatorname{Min} /+}(\mathrm{n}=3)$, $M s t n^{-/}: \because A p c^{M i n /+}(\mathrm{n}=3)$ and $M_{s t n^{-/}}:: A p c^{+/+}(\mathrm{n}=3)$ mice. GAPDH was used as a loading control. GAPDH shown here corresponds to the loading control used for Atrogin-1 (Figure 7.7.2C). The molecular weight markers are represented on the left side of the immunoblots. Immunoblots used for this figure and their corresponding GAPDH loading controls are displayed in Figures 7.7.1 and 7.7.2.

E) Densitometry for Atrogin-1 and MuRF1 normalized to their corresponding GAPDH loading controls for immunoblots represented in Figure 4.7.3D. Significance was assessed by one-way ANOVA followed by Tukey's post-hoc test: ${ }^{*} p<0.05,{ }^{* *} p<0.01,{ }^{* * *} p<0.001$. 

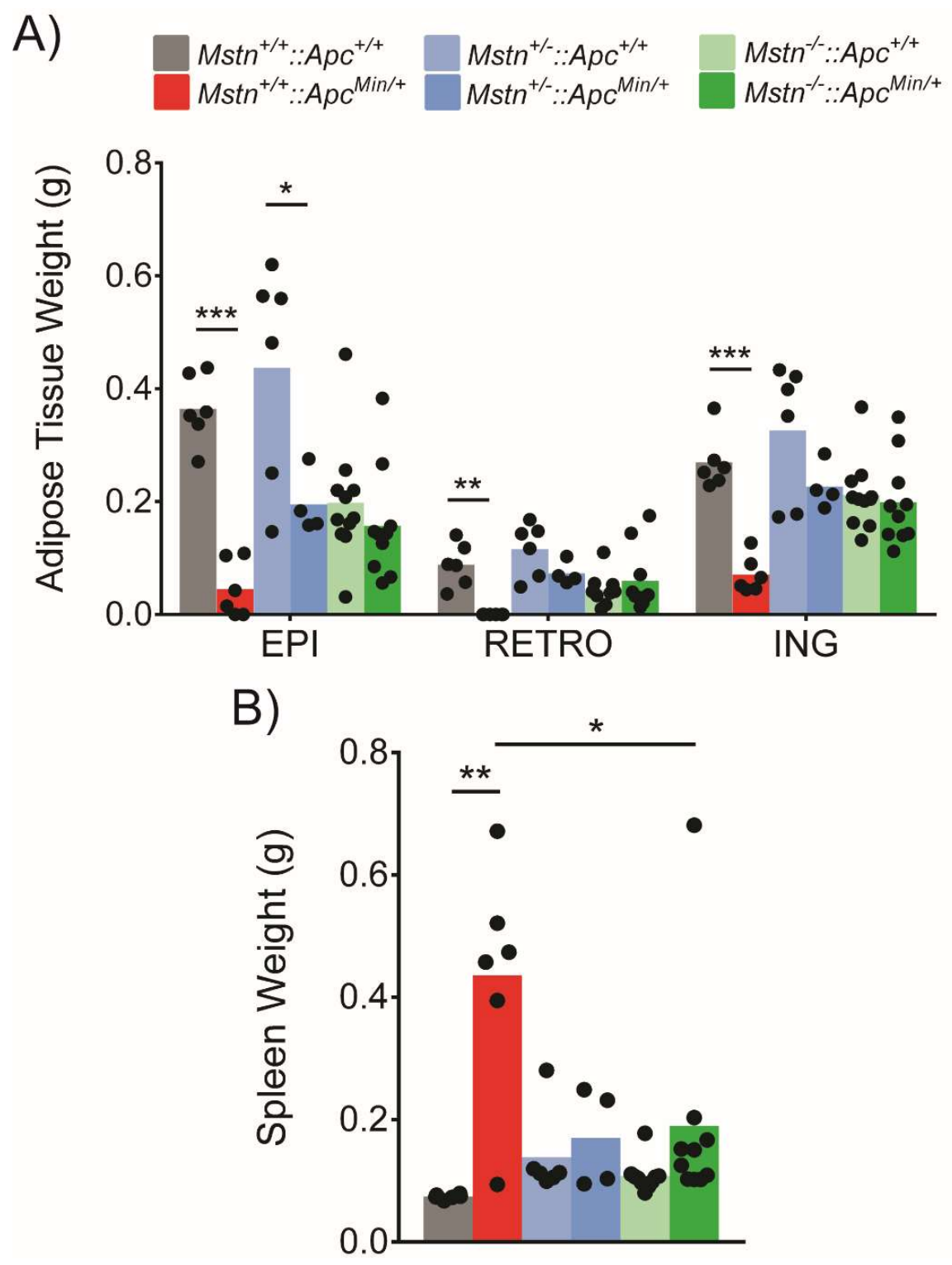

Figure 4.7.4: Genetic ablation of myostatin preserved adipose tissue and spleen mass in 18 week old $A p c^{\mathrm{Min} /+}$ mice.

A) Epididymal (EPI), retroperitoneal (RETRO), and inguinal (ING) adipose tissue weights (grams) for $\operatorname{Mstn}^{+/+}: \because A p c^{M i n /+}(\mathrm{n}=6), \mathrm{Mstn}^{+/} \because \because A p c^{\mathrm{Min} /+}(\mathrm{n}=4)$, and $\operatorname{Msth}^{-/} \because: A p c^{\mathrm{Min} /+}(\mathrm{n}=10)$ mice and their respective wild type controls, $\operatorname{Mstn}^{+/+}: \because \mathrm{Apc}^{+/+}(\mathrm{n}=6), \mathrm{Mstn}^{+/} \because \because \mathrm{Apc}^{+/+}(\mathrm{n}=6)$, and $\mathrm{Mstn}^{-/}: \because \mathrm{Apc}^{+/+}(\mathrm{n}=11)$ mice. Significance was assessed by Student's t-test with Welch correction: ${ }^{*} p<0.05,{ }^{* *} p<0.01$, $* * * p<0.001$

B) Spleen weights (grams) for $M_{s t n}^{+/+}: \because A p c^{M i n /+}(\mathrm{n}=6), M_{s t n}^{+/}: \because A p c^{M i n /+}(\mathrm{n}=4)$, and $\mathrm{Mstn}^{-/-}:: A p c^{\mathrm{Min} /+}$ $(\mathrm{n}=10)$ mice, and their respective wild type controls, $\operatorname{Mstn}^{+/+}: \because A p c^{+/+}(\mathrm{n}=6), M_{s t n}^{+/}: \because A p c^{+/+}(\mathrm{n}=6)$, and $M_{s t n}{ }^{-/} \because: A p c^{+/+}(\mathrm{n}=10)$ mice. Spleen weight for mouse ID $436\left(\mathrm{Mstn}^{-/} \because \because A p c^{+/+}\right)$was not collected. Significance between $\mathrm{Apc}^{\mathrm{Min} /+}$ and $\mathrm{Apc}^{+/+}$mice was assessed by Student's t-test with Welch correction: ${ }^{*} p<0.05,{ }^{* *} p<0.01,{ }^{* * *} p<0.001$. The significance between $\mathrm{Mstn}^{+/+}: \because A p c^{\mathrm{Min} /+}, \mathrm{Mstn}^{+/}$ $\because A p c^{M i n /+}$, and $M_{s t n^{-1}}: \because p c^{M i n /+}$ spleen weights was assessed by one-way ANOVA followed by Tukey's post-hoc test: ${ }^{*} p<0.05,{ }^{*} p<0.01,{ }^{* * *} p<0.001$. 


\subsection{The deletion of myostatin did not alter the intestinal tumor biology of the $\mathrm{Apc}^{\mathrm{Min} /+}$ mouse}

We next examined whether the absence of cancer cachexia in the $\mathrm{Mstn}^{+/-}: \because A p \mathrm{CMin}^{\mathrm{+}}$ and $\mathrm{Msth}^{-/-}$ $\because A p c^{\mathrm{Min} /+}$ mice was as due to a reduced polyp burden in the intestine. To investigate this question, we chose a heterozygote breeding scheme where $\mathrm{Mstn}^{+/-}: \because A p \mathrm{c}^{\mathrm{Min} /+}$ male mice were mated to $\mathrm{Mstn}^{+/}$female mice to generate the following genotypes: $\mathrm{Mstn}^{+/+}: \because \mathrm{Apc}^{\mathrm{Min} /+}, \mathrm{Mstn}^{+/-}$ $\because A p c^{M i n /+}$, and $M_{s t n}{ }^{-/} \because: A p c^{M i n /+}$. The Mstn ${ }^{+/+} \because: A p c^{M i n /+}, M_{s t n}^{+/} \because: A p c^{M i n /+}$, and Mstn ${ }^{-/-} \because A p c^{M i n /+}$ mice were terminated between ages of 20 to 21 weeks and evaluated for their intestinal tumor number and diameter (Figure 4.8.1). Sacrificed body weights and spleen weights for these mice are shown in Figure 7.8.1. According to Figure 4.8.1, we observed no difference in the small intestine tumor number in spite of the loss of myostatin (Figures 4.8.1A). However, the average small intestine tumor diameter measured in the $\mathrm{Mstn}^{-/}: \because A p c^{\mathrm{Min} /+}$ mice was slightly smaller $(15.33 \%)$ than the $M_{s t n^{+/+}}: A p c^{M i n /+}$ controls (Figure 4.8.1B and Table 7.8.1). Furthermore, the average small intestine tumor diameter was significantly smaller in the $\mathrm{Mstn}^{-/-} \because: \mathrm{Apc}^{\mathrm{Min} /+}$ mice when compared to $\mathrm{Mstn}^{+/}: \because A p c^{\mathrm{Min} /+}$ mice (Figure 4.8.1B). There was no difference in the average tumor diameter between $\mathrm{Mstn}^{+/-}: \because \mathrm{Apc}^{\mathrm{Min} /+}$ mice and the $\mathrm{Mstn}^{+/+}: \because \mathrm{Apc}^{\mathrm{Min} /+}$ controls (Figure 4.8.1B). A survey of the literature reveals that the small intestine tumor diameter can also be represented as a group of three ranges $(<1 \mathrm{~mm}, \geq 1$ to $<2 \mathrm{~mm}, \geq 2 \mathrm{~mm})[137,138]$. When the data was grouped into the stated sets we failed to observe a difference in the small intestine tumor diameter amongst the three genotypes (Figures 7.8.2A and 7.8.2B). In addition, the colon tumor number and diameter were approximately the same in all three genotypes (Figures 4.8.1C and 4.8.1D). In conclusion, despite its statistical significance, the difference between the $\mathrm{Mstn}^{-/-}: \because A p c^{\mathrm{Min} /+}$ and the $\mathrm{Mstn}^{+/+}: \because A p c^{\mathrm{Min} /+}$ small intestine tumor diameter was marginal. Furthermore, the loss of significance when the tumor diameter was segmented into three ranges and the fact that there was no difference in the small and large intestine tumor number would suggest no definitive link between myostatin and intestinal tumorigenesis. 


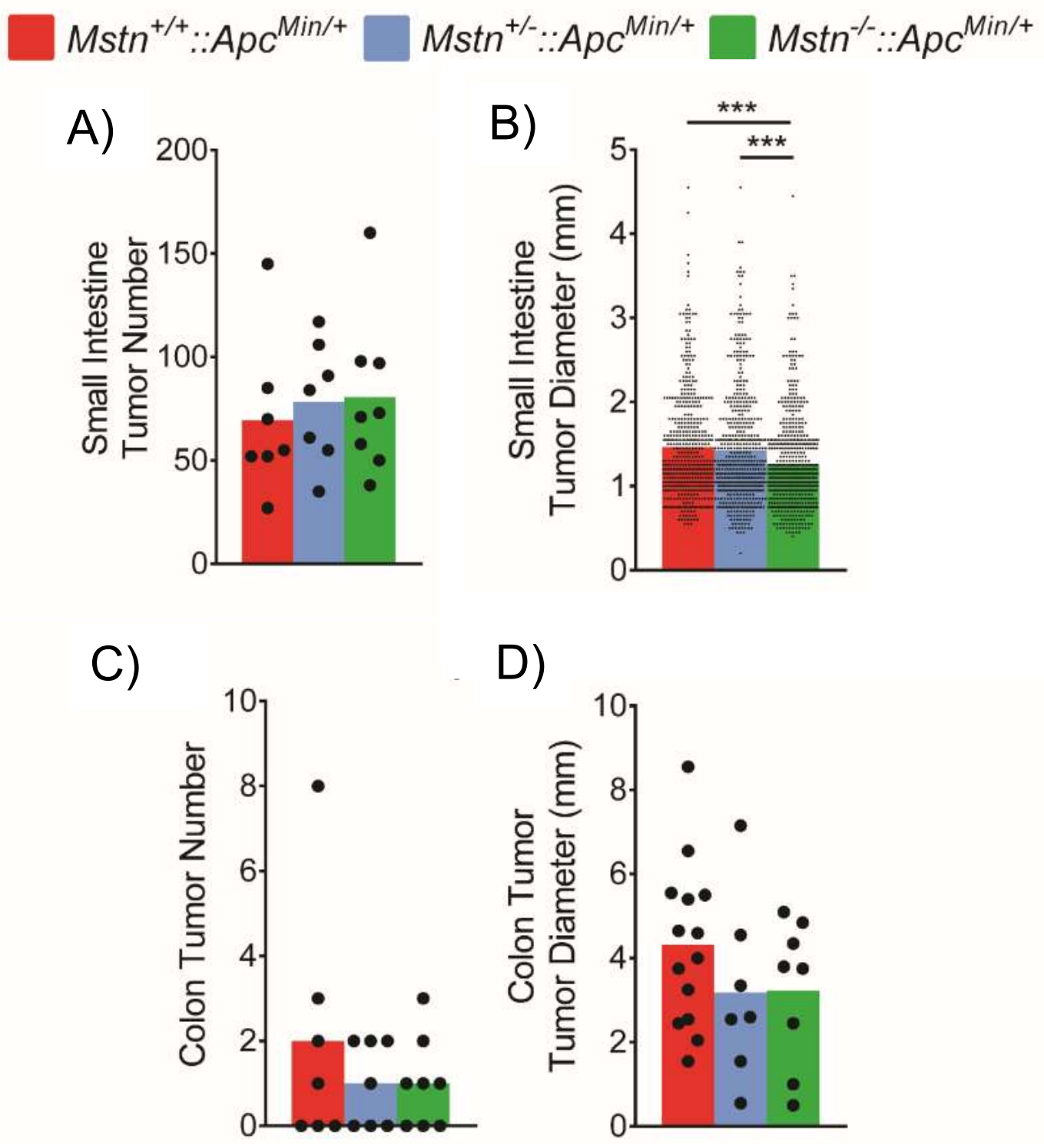

Figure 4.8.1: Genetic ablation of myostatin failed to alter the tumor biology of the $\mathrm{Apc} \mathrm{Min} /+^{\mathrm{N}}$ mouse.

A) Small intestine tumor number for $M_{s t n^{+/+}}: A p c^{\operatorname{Min} /+}(\mathrm{n}=7), M_{s t n}{ }^{+/} \because: A p c^{M i n /+}(\mathrm{n}=7)$, and $M s t n^{-/-}$ $\because A p c^{M i n /+}(\mathrm{n}=8)$ mice. Significance was assessed by one-way ANOVA followed by Tukey's posthoc test: No significant difference was observed.

B) Average small intestine tumor diameter (mm) for $\operatorname{Mstn}^{+/+}: \because A p c^{M i n /+}(\mathrm{n}=7), M_{s t n}^{+/-}: \because A p c^{M i n /+}$ $(\mathrm{n}=7)$, and $\mathrm{Mstn}^{-1}: \because A p c^{\mathrm{Min} /+}(\mathrm{n}=8)$ mice. While $\mathrm{n}$ represents the number of mice used for this experiment, each data point shown in this figure represents the diameter of one small intestinal tumor. Significance was assessed by one-way ANOVA followed by Tukey's post-hoc test: $* p<0.05, * * p<0.01, * * * p<0.001$.

C) Colon tumor number and D) Colon tumor diameter (mm) for $\mathrm{Mstn}^{+/+}: \because \mathrm{Apc}^{\mathrm{Min} /+}$ (n=7), Mstn ${ }^{+/-}$ $\because A p c^{M i n /+}(\mathrm{n}=7)$, and $M_{s t n^{-/}}: \because A p c^{\mathrm{Min} /+}(\mathrm{n}=8)$ mice. For Figure 4.8.1D, $\mathrm{n}$ represents the number of mice used for this experiment, but each data point represents the diameter of a single colon tumor. Significance was assessed by one-way ANOVA followed by Tukey's post-hoc test: No significant difference was observed. Mice used in this experiment were between 20 to 21 weeks of age. 


\subsection{The growth characteristics of $\mathrm{Mstn}^{-/}:: \mathrm{Apc}^{\mathrm{Min} /+}$ cancer organoids}

We next aimed to investigate whether there are any molecular differences exhibited by the polyps generated in the absence of myostatin when compared to the wild type controls. To address this question, we employed the organoid culture technique developed by Professor Hans Clevers group [228]. We isolated and cultured small intestinal tumors from $\mathrm{Mstn}^{+/+} \because: A p c^{\mathrm{Min} /+}, \mathrm{Mstn}^{+/-} \because A p c^{\mathrm{Min} /+}$, and $\mathrm{Mstn}^{-/-}: \because A p c^{\mathrm{Min} /+}$ mice. These mice were generated from the heterozygote breeding scheme described in section 4.8. According to Figure 4.9.1A, organoids isolated from $\mathrm{Mstn}^{-/} \because: A p c^{\mathrm{Min} /+}$ mice were smaller and darker than the $\mathrm{Mstn}^{+/+}: \because A p c^{\mathrm{Min} /+}$ and $\mathrm{Mstn}^{+/}: \because A p c^{\mathrm{Min} /+}$ cancer organoids (Figure 4.9.1A). The preponderance of these smaller organoids noted in the $\mathrm{Mstn}^{-/}: \because A p c^{\mathrm{Min} /+}$ mice might not be due to apoptosis, as cleaved caspase-3 levels in the $M_{s t n^{-1}} \because A p c^{M i n /+}$ organoids were similar to both $\mathrm{Mstn}^{+/+}: \because A p c^{\mathrm{Min} /+}$ and Msth $^{+/-}: \because A p c^{\mathrm{Min} /+}$ cancer organoids (Figures 4.9.1B, 4.9.1C, Figures 7.9.1 and 7.9.2). Furthermore, the organoids collected from these three genotypes demonstrated similar levels of Ki67 and PCNA messenger RNA, two markers of proliferation (Figure 4.9.1C). The gene expression of stem cell markers such as Lgr5 and Bmil in these three groups were also relatively unchanged (Figure 4.9.1C). Conversely, $\mathrm{Mstn}^{+/}: \because \mathrm{Apc}^{\mathrm{Min} /+}$ organoids demonstrated a significantly lower c-Myc transcript level compared to the wild type control (Figure 4.9.1C). This reduction in c-Myc gene expression levels was not observed in the Mstn ${ }^{\prime}: \because A p c^{M i n /+}$ organoids when compared to the wild type $\mathrm{Mstn}^{+/+}: \because A p c^{\mathrm{Min} /+}$ organoids (Figure 4.9.1C). 

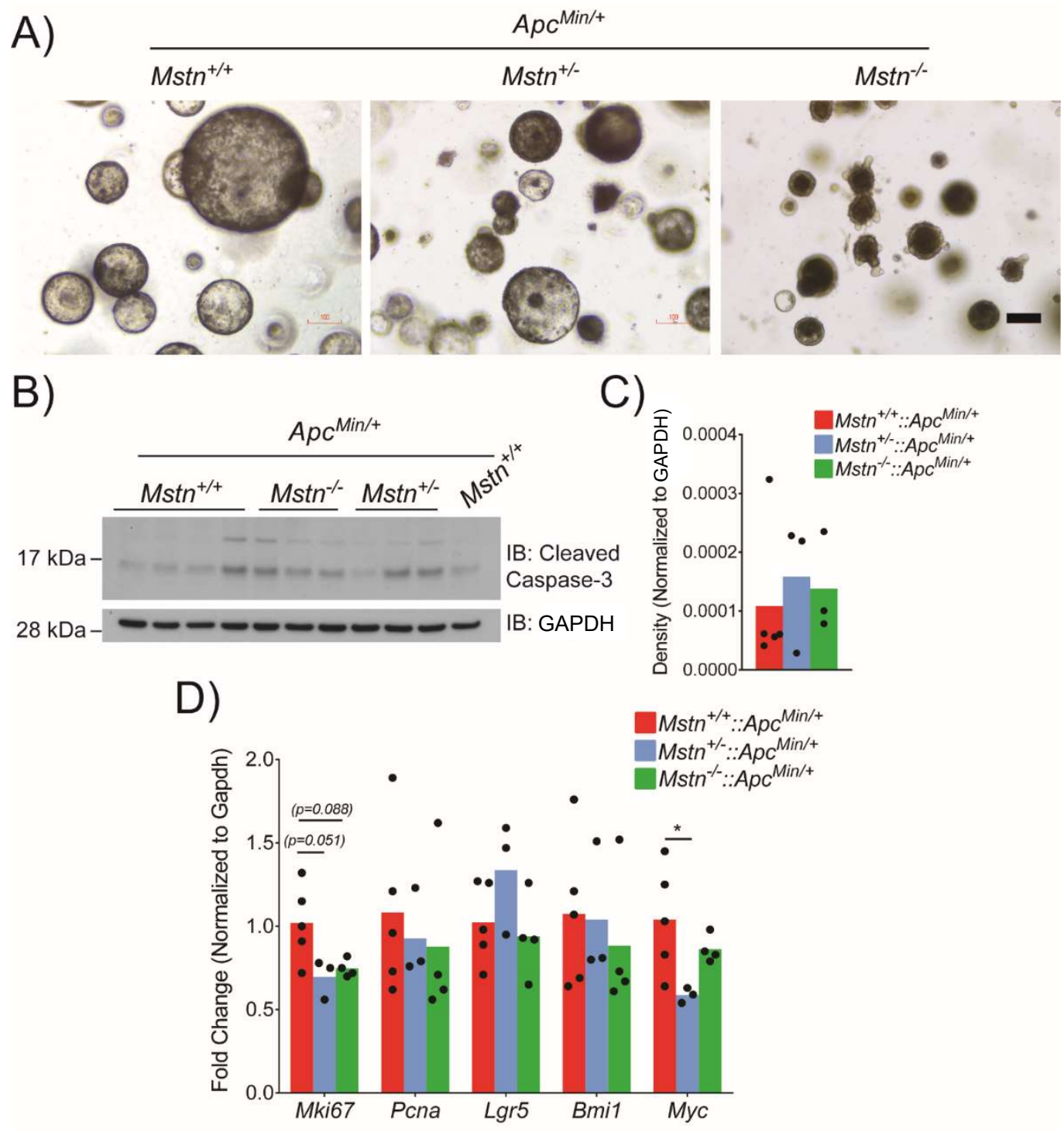

Figure 4.9.1: The growth characteristics of $\mathrm{Mstn}^{+/+}:: \mathrm{Apc}^{\mathrm{Min} /+}, \mathrm{Mstn}^{+/-}:: \mathrm{Apc}^{\mathrm{Min} /+}$, and Mstn- :Apc ${ }^{\mathrm{Min} /+}$ small intestine cancer organoids.

A) Representative bright field images of $\mathrm{Mstn}^{+/+}: \because A p c^{\mathrm{Min} /+}, \mathrm{Mstn}^{+/} \because: A p c^{\mathrm{Min} /+}$, and $\mathrm{Msth}^{-/} \because: A p c^{\mathrm{Min} /+}$ cancer organoids at $4 \mathrm{X}$. Scale bar represents $100 \mu \mathrm{m}$.

B) Immunoblot for cleaved caspase-3 for $\mathrm{Mstn}^{+/+}: \because A p c^{\mathrm{Min} /+}(\mathrm{n}=5), \mathrm{Mstn}^{+/}: \because A p c^{\mathrm{Min} /+}(\mathrm{n}=3)$, and $M_{s t n^{-1}}: \because A p c^{M i n /+}(\mathrm{n}=3)$ cancer organoids. GAPDH was used as a loading control. GAPDH shown here corresponds to the loading control used cleaved-caspase 3 . The molecular weight markers are represented on the left side of the immunoblots. Immunoblot used for this figure and their corresponding GAPDH loading control are displayed in Figures 7.9.1 and 7.9.2.

C) Densitometry for cleaved-caspase 3 normalized to their corresponding GAPDH loading controls for immunoblots represented in Figure 4.9.1B. Significance was assessed by one-way ANOVA followed by Tukey's post-hoc test: No significant difference was observed.

D) Relative gene expression of Ki67 (Mki67), PCNA (Pcna), Lgr5 (Lgr5), Bmi1 (Bmil), and cMyc $(M y c)$ in Mstn $^{+/+}: \because A p c^{M i n /+}(\mathrm{n}=5)$, Mstn $^{+/}: \because A p c^{M i n /+}(\mathrm{n}=3)$, and $\operatorname{Mstn}^{-/} \because: A p c^{M i n /+}(\mathrm{n}=4)$ cancer organoids. Gene expression was normalized to Gapdh. Significance was assessed by one-way ANOVA followed by Tukey's post-hoc test: ${ }^{*} \mathrm{p}<0.05, * * \mathrm{p}<0.01, * * * \mathrm{p}<0.001 . \Delta \mathrm{Ct}$ was used to calculate significance. 


\subsection{0 $\mathrm{Mstn}^{-/-}$mice were resistant to colitis-induced colorectal carcinogenesis}

We were unable to conclusively identify a link between myostatin and intestinal tumor biology using the $A p c^{\mathrm{Min} /+}$ mouse model. To further investigate the relationship between myostatin and intestinal tumorigenesis, we employed the AOM/DSS model of colitis-induced colorectal carcinogenesis to examine the susceptibility of $\mathrm{Mstn}^{-/}$mice to colorectal tumors. The AOM/DSS procedure was performed on male $\mathrm{Mstn}^{+/+}$and $\mathrm{Mstn}^{-/}$mice that were generated by an $\mathrm{Mstn}^{+/-}$male and $\mathrm{Mstn}^{+/-}$female heterozygote breeding scheme.

As expected, the administration of $2.5 \%$ DSS into the drinking water resulted the loss of body mass in both $\mathrm{Mstn}^{+/+}$and $\mathrm{Mstn}^{-/}$mice (Figure 4.10.1A). Although the two genotypes consumed a relatively similar volume of $2.5 \%$ DSS per cycle, the extent of mass loss appeared to be greater in the $\mathrm{Mstn}^{+/+}$mice compared to the $\mathrm{Mstn}^{-/-}$mice (Figures 4.10.1B and 4.10.1C). Mice were sacrificed between 9 to 11 weeks after the administration of AOM for the assessment of colorectal tumors (Figure 4.10.2). Quite surprisingly, we recorded a significantly larger number of colorectal tumors in the $\mathrm{Mstn}^{+/+}$mice compared to the $\mathrm{Mstn}^{-/-}$mice (Figures 4.10.2A, 4.10.2B, Figures 7.10.1 and 7.10.2). In other words, $\mathrm{Mstn}^{-/}$mice exhibited an almost 75\% reduction in colorectal tumor number when compared to their wild type controls (Figures 4.10.2A, 4.10.2B, Figures 7.10.1 and 7.10.2). $\mathrm{Mstr}^{-/}$mice also displayed a significantly smaller colorectal tumor diameter than the $\mathrm{Mstn}^{+/+}$mice (Figure 4.10.2C). While the resistance to weight loss induced by $2.5 \%$ DSS consumption observed in the $\mathrm{Msth}^{-/-}$mice is an indicator of reduced inflammation, we failed to observe a similar decrease in other colitis-associated markers in mice devoid of myostatin when compared to the wild type controls (Figures 4.10.1F, 4.10.2D, and 4.10.2E). In particular, the two genotypes failed to demonstrate any difference in the spleen weight, colon length, or uncleared colon weights (Figures 4.10.1F, 4.10.2D, and 4.10.2E). Data for the sacrificed body weight, skeletal muscle mass, and tibia length are presented in Figures 4.10.1D, 4.10.1E, Table 7.10.1, and Table 7.10.2. 

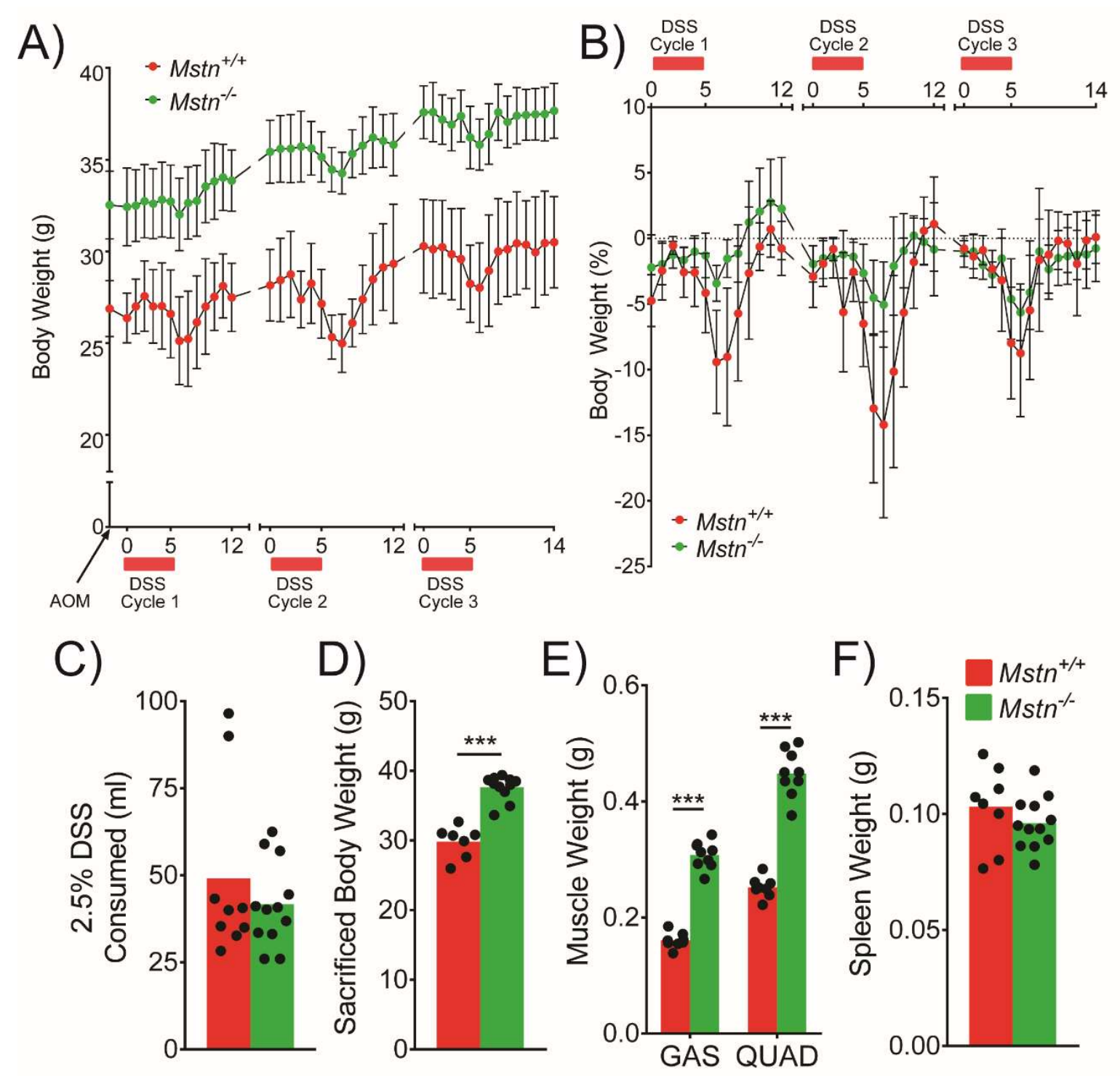

Figure 4.10.1: Body weights for male $\mathrm{Mstn}^{+/+}$and $\mathrm{Mstn}^{-/}$mice subjected to the AOM/DSS model of colitis-induced colorectal carcinogenesis.

A) Body weights (grams) for $\mathrm{Mstn}^{+/+}(\mathrm{n}=6-7)$ and $\mathrm{Mstn}^{-/}(\mathrm{n}=9)$ mice during the course of the AOM/DSS experimental procedure. Error bars denote $\pm 95 \%$ CI. The body weight of one $\mathrm{Mstn}^{+/+}$ mouse was not measured on one day due to an administrative error.

B) Body weight change (\%) for $\mathrm{Mstn}^{+/+}(\mathrm{n}=6-7)$ and $\mathrm{Mstn}^{-/-}(\mathrm{n}=9)$ mice during the course of the AOM/DSS experimental procedure. Percentage change in body weight was calculated by dividing the body weight by the maximum body weight observed during the 5 day $2.5 \%$ DSS treatment regime. Error bars denote $\pm 95 \% \mathrm{CI}$. The body weight of one $M s t n^{+/+}$mouse was not measured on one day due to an administrative error.

C) The amount of $2.5 \%$ DSS consumed $(\mathrm{ml})$ per mouse per cycle by $\mathrm{Mstn}^{+/+}(\mathrm{n}=9)$ and $\mathrm{Msth}^{-/-}$ $(n=12)$ mice, where $n$ represents number of cages. Significance was assessed by Student's t-test with Welch correction: No significant difference was observed.

D) Sacrificed body weights (grams) for $\mathrm{Mstn}^{+/+}(\mathrm{n}=7)$ and $\mathrm{Msth}^{-/}(\mathrm{n}=11)$ mice. Significance was assessed by Student's t-test with Welch correction: ${ }^{*} p<0.05, * * p<0.01, * * * p<0.001$.

E) Gastrocnemius (GAS) and quadriceps (QUAD) weights (grams) for $M_{s t n}^{+/+}(\mathrm{n}=8)$ and $\mathrm{Msth}^{-/}$ $(\mathrm{n}=9)$ mice. Significance was assessed by Student's t-test with Welch correction: ${ }^{*} p<0.05$, $* * p<0.01, * * * p<0.001$.

F) Spleen weights (grams) for $M s n^{+/+}(\mathrm{n}=8)$ and $M s t n^{-/-}(\mathrm{n}=12)$ mice. Significance was assessed by Student's t-test with Welch correction: ${ }^{*} p<0.05,{ }^{*} p<0.01,{ }^{* * *} p<0.001$. 


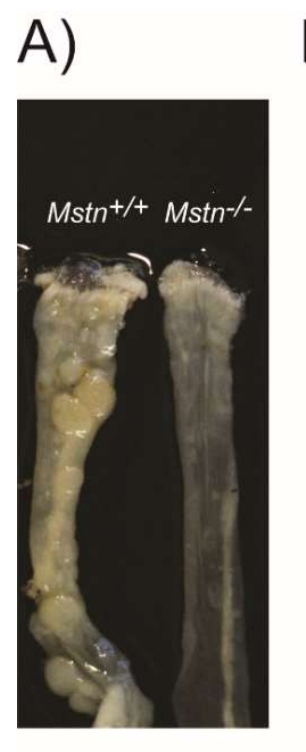

B)
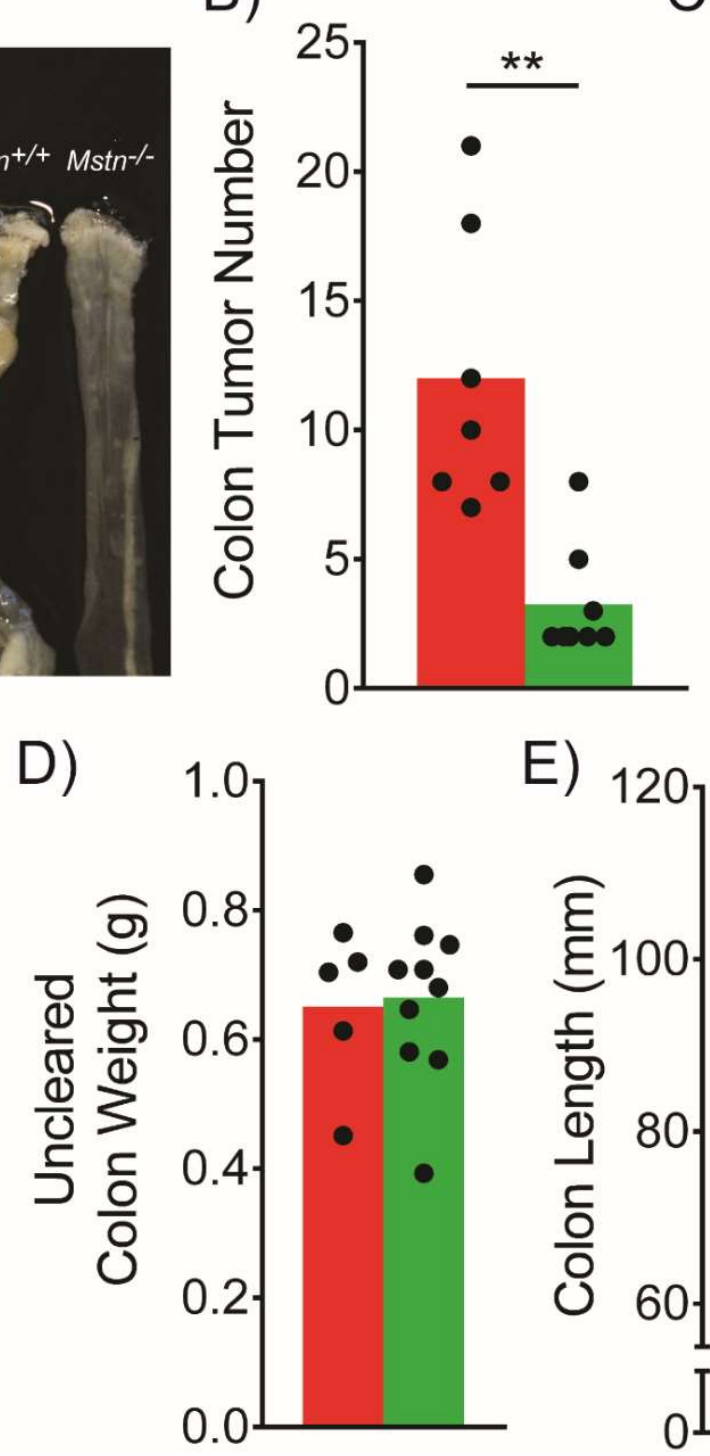

C)

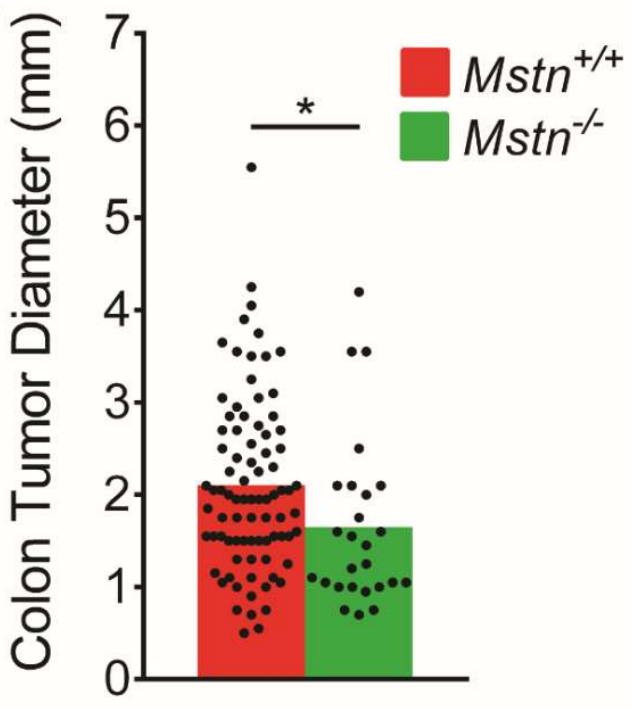

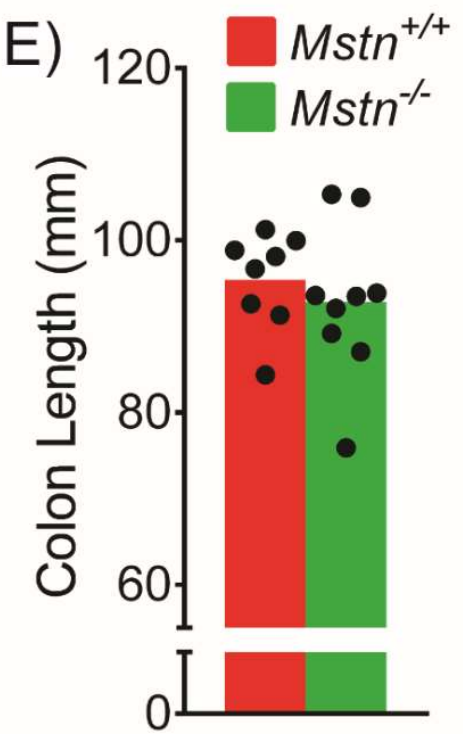

Figure 4.10.2: Absence of myostatin suppressed colitis-induced colorectal tumorigenesis. A) Representative image of $10 \% \mathrm{NBF}$ fixed $\mathrm{Mstn}^{+/+}$and $\mathrm{Msth}^{-/}$colons. The distal region of the colon is represented in this figure. Please refer to Figures 7.10.1 and 7.10.2 for the images of the colons used for analysis.

B) Colon tumor number for $\mathrm{Mstn}^{+/+}(\mathrm{n}=7)$ and $\mathrm{Mstn}^{-/-}(\mathrm{n}=8)$ mice. Significance was assessed by Student's t-test with Welch correction: $* p<0.05, * * p<0.01, * * * p<0.001$.

C) Average colon tumor diameter $(\mathrm{mm})$ for $\mathrm{Mstn}^{+/+}(\mathrm{n}=7)$ and $\mathrm{Msth}^{-/}(\mathrm{n}=8)$ mice. For the colon tumor diameter, $\mathrm{n}$ represents the number of mice, but each data point represents the diameter of a single colon tumor. Significance was assessed by Student's t-test with Welch correction: ${ }^{*} p<0.05$, $* * p<0.01, * * * p<0.001$.

D) Colon length $(\mathrm{mm})$ for $\mathrm{Mstn}^{+/+}(\mathrm{n}=8)$ and $\mathrm{Mstn}^{-/-}(\mathrm{n}=9)$ mice. Significance was assessed by Student's t-test with Welch correction: $* p<0.05, * * p<0.01, * * * p<0.001$.

E) Uncleared colon weight $(\mathrm{mm})$ for $\mathrm{Mstn}^{+/+}(\mathrm{n}=5)$ and $\mathrm{Mstn}^{-/-}(\mathrm{n}=10)$ mice. Significance was assessed by Student's t-test with Welch correction: ${ }^{*} p<0.05,{ }^{* *} p<0.01,{ }^{* * *} p<0.001$. 


\subsection{The intestinal biology of the $\mathrm{Mstn}^{-/}$mice}

The resistance to colitis-induced colorectal carcinogenesis exhibited by $\mathrm{Msth}^{-/-}$mice suggested that the absence of myostatin may alter normal intestinal homeostasis. To gain an understanding of the intestinal biology of the $\mathrm{Msth}^{-/}$mouse, we measured the intestinal lengths and weights of male $\mathrm{Mstn}^{+/+}, \mathrm{Mstn}^{+/-}$, and $\mathrm{Mstn}^{-/-}$mice (Figure 4.11.1). According to Figure 4.11.1, $\mathrm{Mstn}^{-/}$mice did not display any difference in small intestine and colon length compared to the $\mathrm{Mstn}^{+/+}$mice (Figure 4.11.1A and 4.11.1D). $\mathrm{Mstn}^{+/-}$mice exhibited a significantly longer small intestine length compared to $\mathrm{Mstn}^{-/}$mice (Figure 4.11.1A). The uncleared and cleared intestinal weights were relatively similar in all three genotypes (Figures 4.11.1B, 4.11.1C, 4.11.1E, and 4.11.1F). In addition, $M_{s t n^{+/+}} \because A p c^{M i n /+}, M_{s t n^{+/-}} \because A p c^{M i n /+}$ and $M_{s t n^{-/}} \because A p c^{M i n /+}$ mice displayed no significant differences in their intestinal length and uncleared colon weight (Figure 7.11.1). However, $\mathrm{Mstn}^{-/}: \because A p c^{\mathrm{Min} /+}$ mice exhibited a lighter uncleared small intestine weight when compared to $\mathrm{Mstn}^{+/+}: \because A p c^{\mathrm{Min} /+}$ mice (Figure 7.11.1B). 

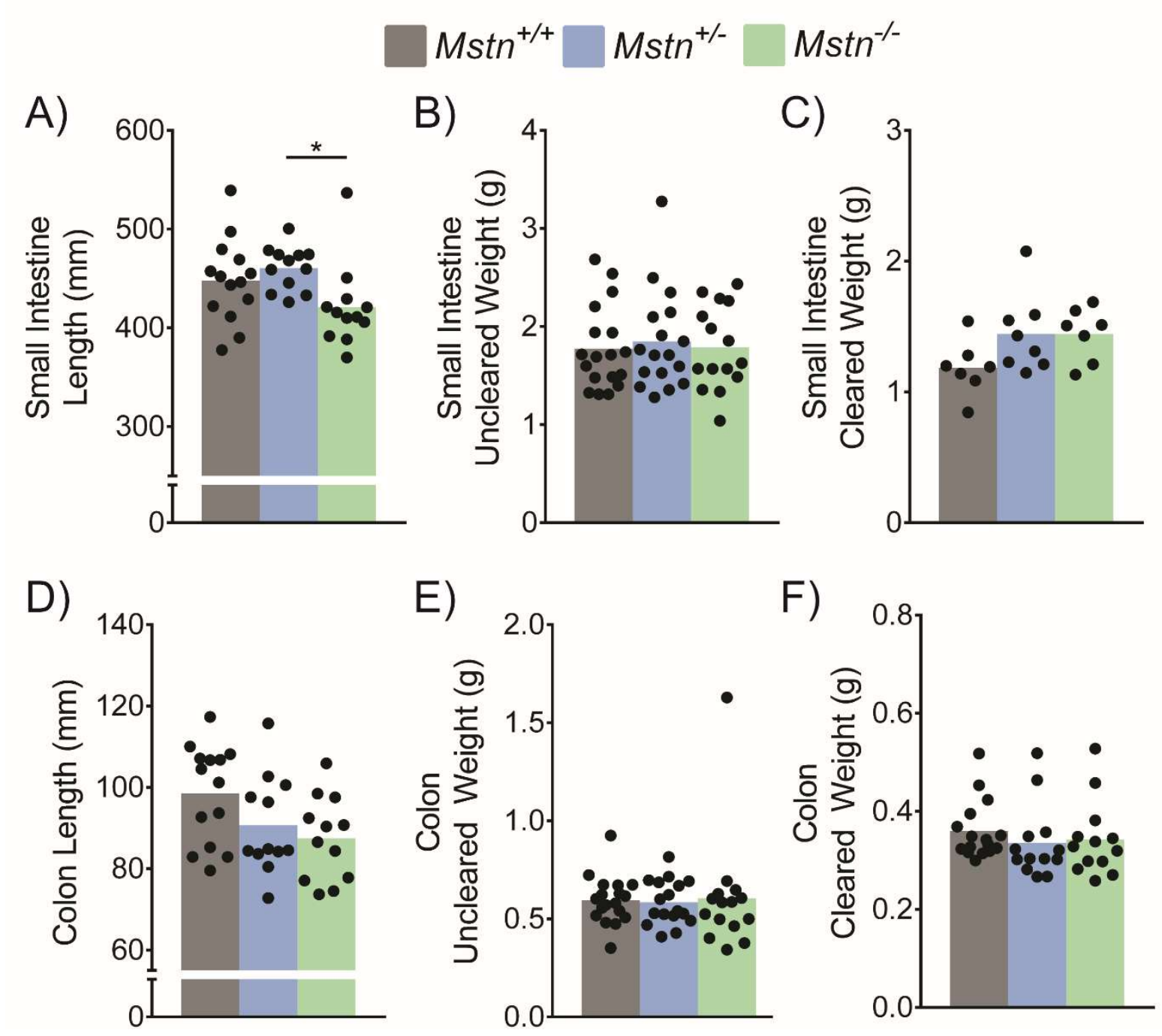

Figure 4.11.1: Intestinal biology of $\mathrm{Mstn}^{+/+}, \mathrm{Mstn}^{+/}$, and $\mathrm{Mstn}^{-/}$mice.

A) Small intestine length (mm) of $\operatorname{Mstn}^{+/+}(\mathrm{n}=14), \mathrm{Mstn}^{+/}(\mathrm{n}=12)$, and $\mathrm{Mstn}^{-/}(\mathrm{n}=12)$ mice. Mice were between 18 to 21 weeks old. Significance was assessed by one-way ANOVA followed by Tukey's post-hoc test: ${ }^{*} \mathrm{p}<0.05,{ }^{* *} \mathrm{p}<0.01,{ }^{* * *} \mathrm{p}<0.001$.

B) Uncleared small intestine weight (grams) of $\operatorname{Mstn}^{+/+}(\mathrm{n}=18), \mathrm{Mstn}^{+/-}(\mathrm{n}=17)$, and $\operatorname{Msth}^{-/}(\mathrm{n}=15)$ mice. Mice were 18 to 22 weeks old. Significance was assessed by one-way ANOVA followed by Tukey's post-hoc test: No significant difference was observed.

C) Small intestine cleared weight (grams) of $\mathrm{Mstn}^{+/+}(\mathrm{n}=7), \mathrm{Mstn}^{+/-}(\mathrm{n}=8)$, and $\mathrm{Mstn}^{-/}(\mathrm{n}=7)$ mice. Mice were 18 to 22 weeks old. Significance was assessed by one-way ANOVA followed by Tukey's post-hoc test: No significant difference was observed.

D) Colon length $(\mathrm{mm})$ of $\mathrm{Mstn}^{+/+}(\mathrm{n}=14), \mathrm{Mstn}^{+/}(\mathrm{n}=12)$, and $\operatorname{Msth}^{-/}(\mathrm{n}=12)$ mice. Mice were between 18 to 21 weeks old. Significance was assessed by one-way ANOVA followed by Tukey's post-hoc test: No significant difference was observed.

E) Uncleared colon weight (grams) of $\mathrm{Mstn}^{+/+}(\mathrm{n}=18), \mathrm{Mstn}^{+/}(\mathrm{n}=17)$, and $\mathrm{Msth}^{-/-}(\mathrm{n}=15)$ mice. Mice were 18 to 22 weeks old. Significance was assessed by one-way ANOVA followed by Tukey's post-hoc test: No significant difference was observed.

F) Cleared colon weight (grams) of $\operatorname{Mstn}^{+/+}(\mathrm{n}=16), \mathrm{Mstn}^{+/}(\mathrm{n}=13)$, and $\mathrm{Msth}^{-/}(\mathrm{n}=13)$ mice. Mice were 18 to 22 weeks old. Significance was assessed by one-way ANOVA followed by Tukey's post-hoc test: No significant difference was observed. 


\section{Discussion}

The elevated expression of myostatin is a salient feature of cancer cachexia and several other metabolic pathologies ranging from diabetes and insulin resistance to heart failure and disuse atrophy [149, 280, 290-293]. Using the $A p c^{\mathrm{Min} /+}$ mouse model of intestinal tumorigenesis, we have documented the upregulation of myostatin gene expression in the skeletal muscles of cachectic $A p c^{\mathrm{Min} /+}$ mice (Figure 4.4.1, Tables 7.1.1 and 7.1.2). Furthermore, the loss of skeletal muscle mass and function in cachectic $A p c^{\mathrm{Min} /+}$ mice was marginally rescued by the administration of sActRIIB, an antagonist of myostatin (Figures 4.6.1-4.6.6). The link between muscle atrophy and myostatin in the $A p{ }^{\mathrm{Min} /+}$ mouse was further supported by the delayed onset of cancer cachexia in $\mathrm{Apc}^{\mathrm{Min} /+}$ mice that were devoid of myostatin (Figure 4.7.1-4.7.3). Finally, in spite of using two models of colorectal carcinogenesis, the work presented in this study was unable to establish a convincing relationship between myostatin and intestinal tumorigenesis (Figures 4.8.1C, 4.10.1, and 4.10.2).

\subsection{The $A p c^{\mathrm{Min} /+}$ model of cancer cachexia}

The role of myostatin in cancer cachexia has been studied in several tumor explantation models $[63,201,202]$. One of the most well characterized model of experimental cancer cachexia is the C26 colon cancer model. While this system has contributed invaluable information regarding the pathology of cancer cachexia, the $\mathrm{C} 26$ model as well as other tumor explantation methods have several shortcomings. A principal disadvantage of this model is that the tumor mass can account for $10 \%$ of the body weight [294]. Cancer cachexia in humans is observed in spite of a much lower tumor burden ( $<1 \%$ of body mass) [295]. Another limitation of the C26 model is the dependency on a mixed background mouse, $\mathrm{CD} 2 \mathrm{~F} 1$, generated from the $\mathrm{F} 1$ progeny of DBA2/J and BALB/c mice [201]. C26 cells do not grow in C57BL/6 mice, rendering the study of C26-induced cancer cachexia in the $M s t n^{-/-}$background unfeasible [201].

The $A p c^{\mathrm{Min} /+}$ mouse has been extensively used to examine intestinal and colonic tumorigenesis [255]. Developed by Moser et al., the $A p c^{\mathrm{Min} /+}$ mouse manifests multiple polyps along the small and large intestine [252]. Professor Carson's group at the University of South Carolina pioneered the use of the $A p c^{\mathrm{Min} /+}$ mouse for the study of cancer cachexia [253, 296]. The results presented in this report provides further support for the $A p c^{\mathrm{Min} /+}$ mouse as a faithful model of cancer cachexia. The $\mathrm{Apc}^{\mathrm{Min} /+}$ male mice in our colony exhibited a precipitous decrease in body 
weight between 14 weeks to 18 weeks of age (Figure 4.1.1). This loss of body weight was associated with the atrophy of the skeletal muscle and adipose tissue depots (Figures 4.2.1 and 4.2.2). We did observe some variability in the myofiber cross-sectional area between the noncachectic and wild type mice, though this difference was insignificant (Figure 4.2.1C). We believe that this variability could be due to the lack of replicates, and as such, future experiments should include TA myofiber cross-sectional area measurements from at least five mice. In addition, a potential improvement to this study would have been to measure the grip strength of the mice and determine whether there was a correlation between the severity of cancer cachexia and muscle function. According to the findings presented in this report, the increasing severity of cancer cachexia in the $\mathrm{Ap} \mathrm{c}^{\mathrm{Min} /+}$ mouse was also accompanied by the increased expression of the catabolic E3 ligases, Atrogin-1 and MuRF1 (Figures 4.3.1 and 4.3.2). This likely elevation in activity of the UPP could be correlated to the elevated amounts of active, de-phosphorylated FoxO3 transcription factor, a principal regulator of the skeletal muscle atrophy program (Figure 4.3.2) [116].

While we have observed a correlation between the increased expression of key UPP components and the severity of cancer cachexia, it must be stressed that further experiments should be conducted to ascertain the activity of this pathway as the $A p c^{\mathrm{Min} /+}$ mouse develops a cachectic phenotype. One such improvement to this report would be to quantify the rate of proteolysis through a flurometric free tyrosine release assay in the skeletal muscles of cachectic $\mathrm{Apc}^{\mathrm{Min} /+}$ mice [297]. In fact, using the free tyrosine release assay, White and colleagues have demonstrated that protein degradation was increased as the $\mathrm{Apc}^{\mathrm{Min} /+}$ mouse manifested cachexia [297]. Furthermore, a UPP inhibitor such as MG 132 could be inoculated into cachectic $\mathrm{Apc}^{\mathrm{Min} / \mathrm{+}}$ mice to determine whether the attenuation of this degradative pathway could rescue the loss of skeletal muscle mass and function [298]. Finally, performing a pull-down assay to quantify the extent of ubiquitination on bona fide substrates of the E3 ligases, such as the structural proteins and MyoD, could also reveal the activity of the UPP during cancer cachexia in the $A p c^{\mathrm{Min} /+}$ mouse [5].

The regular progression of intestinal tumorigenesis in the $A p c^{\mathrm{Min} /+}$ mouse is a purported advantage of this model compared to other methods of cancer cachexia induction in rodents [137, 138, 279, 299]. However, we noted that the onset of cancer cachexia in this model was heterogeneous (Figure 4.1.1 and Table 7.1.1). We could surmise that the variability in the induction of cancer cachexia arose from the stochastic nature of intestinal tumor formation in 
the $A p c^{\mathrm{Min} /+}$ mouse. In fact, we have observed that $A p c^{\mathrm{Min} /+}$ mice exhibited a variable tumor multiplicity in the small intestine (Figure 4.5.2A). Could the intestinal tumor burden be correlated to the severity of cancer cachexia in the $\mathrm{Apc}^{\mathrm{Min} /+}$ mouse? A report by Baltgalvis and co-workers have in fact highlighted that the $\mathrm{Apc}^{\mathrm{Min} /+}$ mice became severely cachectic once the tumor burden in the small intestine and colon exceeded a threshold [138]. The relationship between the intestinal tumor burden and the onset of cancer cachexia could be reinforced by our observation that the polyps are a rich source of IL-6 and TNF $\alpha$ mRNA (Figures 4.5.1 and 7.5.1). Although another report has confirmed a similar enrichment of IL-6 and TNF $\alpha$ mRNA in the intestinal tumors of $\mathrm{Apc}^{\mathrm{Min} /+}$ mice, an ELISA test should be accomplished to gain a clearer insight into the expression level of these pro-cachectic cytokines [300]. Nevertheless, IL-6 and TNF $\alpha$ are not only potent inducers of cancer cachexia, but are also stimulators of myostatin gene expression [285-287]. However, our report failed to observe a strong correlation between the intestinal tumor burden and the severity of cancer cachexia (Figure 4.5.2). Our results could suggest that there are other variables such as the onset of anemia and anorexia, which could potentially act together with the intestinal tumor burden to influence the expression of myostatin in the skeletal muscle of cachectic $A p c^{\mathrm{Min} /+}$ mice.

\subsection{The role of myostatin in cancer cachexia}

Myostatin over-expression is a critical feature of several muscle wasting pathologies [301]. We recorded that myostatin gene expression was upregulated during the intermediate to severe stages of cancer cachexia in the $A p c^{\mathrm{Min} /+}$ mouse (Figure 4.4.1). A large variation in the relative transcript levels of myostatin was noted in the severely cachectic $A p c^{\mathrm{Min} /+}$ mice (Figures 4.4.1, 7.4.1, and 7.4.2). This heterogeneity in the myostatin transcript levels could have been due to the relative instability of certain housekeeping genes we have used in this study (Figures 4.4.1, 7.4.1, and 7.4.2). However, if we consider the observation that early cachectic $A p c^{\mathrm{Min} /+}$ mice failed to display elevated amounts of myostatin gene expression and that moderately cachectic mice consistently exhibited increased amounts of myostatin mRNA, it is possible that the transcription of this growth factor may only occur during periods of rapid remodeling of the skeletal muscle. Furthermore, our results may explain why certain studies have reported variability in the expression of myostatin in human cancer cachexia samples [149, 204-207]. In other words, careful characterization of the extent of cancer cachexia in human patients using the similar parameters described in this report may yield a clearer indication of the expression status of this growth hormone. 
The use of antibodies to detect myostatin in the skeletal muscle and serum would have greatly improved this aspect of the study. The overt reliance on gene expression data, though highly encouraging, overlooks other considerations such as the translation rate of this particular growth hormone. Moreover, like other TGF- $\beta$ molecules, myostatin activity is dependent on the furin-like proconvertases which cleaves the homodimeric precursor at the pro-domain and the active region $[152,302]$. Proteolysis alone is insufficient as the non-covalent association of the pro-domain to the $\mathrm{C}$-terminal domain, termed as the latent complex, can suppress the biological activity of myostatin [144, 303]. In fact, the cleavage of the pro-domain by the proteolytic activity of the BMP-1/Tolloid family of metalloproteinases liberates the mature myostatin from the latent complex [160]. Furthermore, myostatin activity is tightly regulated by other extracellular binding proteins such as FSTL3/FLRG protein, the WAP, Kazal, immunoglobulin, Kunitz and NTR domain-containing protein 1 and 2 (WFIKKN1 and WFIKKN2) [303, 304]. Given the nuances in the regulation of myostatin function, it is currently unknown how much biologically active myostatin maybe present during the course of cancer cachexia in the $A p c^{\mathrm{Min} /+}$ mouse.

It must be disclosed that attempts were made to validate the protein levels of myostatin in the skeletal muscle and serum of cachectic $A p c^{\mathrm{Min} /+}$ mice (Figures 7.12.1 - 7.12.8). After using two brands of myostatin antibodies, we failed to observe any change in the myostatin levels during the course of cachexia (Figures 7.12.1, 7.12.3 - 7.12.6). In addition, two ELISA kits were used to detect the presence of circulating myostatin levels in the stratified cancer cachexia samples (Figures 7.12.2B and 7.12.2C). The Immundiagnostik MSTN ELISA kit yielded a result that was antithetical to the transcription amounts of myostatin during cancer cachexia in the $A p c^{\mathrm{Min} /+}$ mouse (Figure 7.12.2C). Conversely, the R\&D Systems ELISA kit yielded mixed results (Figure 7.12.2B).

The difference between the mRNA and protein levels of myostatin in the skeletal muscles of $A p c^{M i n /+}$ mice undergoing cancer cachexia could be due to several reasons. As highlighted previously, one plausible explanation is that the rate of gene transcription of myostatin is uncoupled from the rate of protein translation. In other words, the increased amount of myostatin mRNA cannot be efficiently translated into functional myostatin. Conversely, myostatin is a secreted hormone [150]. Thus, if there is no difference in the rate of transcription and translation, the relatively unchanged amount of myostatin protein levels may suggest an increased rate of secretion from the muscles into circulation. While this may be an attractive 
explanation to reconcile the difference between the protein and mRNA expression of myostatin, this reason falls short because of the inconclusive ELISA results (Figures 7.12.2B and 7.12.2C). A reason why our ELISA experiments yielded mixed outcomes was likely due to the quality of the serum collected. The $\mathrm{Apc}^{\mathrm{Min} /+}$ mouse undergoes anemia due to the tumor burden from the intestine [255]. It is currently unknown how the quality of the serum collected from anemic $A p c^{\mathrm{Min} /+}$ mice may affect the reliability of the myostatin ELISA results.

The principle issue with antibody based methods of detecting this growth hormone stems from its remarkable similarity to GDF-11 and other TGF- $\beta$ signaling molecules. In fact, 11 amino acids distinguish the mature GDF-11 from myostatin [305]. Thus, a myostatin antibody designed against the mature region would likely detect the presence of GDF-11. We believe this issue was highlighted when we detected the presence of an immunoreactive band in $\mathrm{Mstn}^{-}$ /- skeletal muscles using the R\&D Systems myostatin antibody (Figures 7.12.2A, 7.12.7, and 7.12.8). In addition, using the same R\&D Systems myostatin ELISA kit, Rodgers and Eldridge detected circulating myostatin in $\mathrm{Msth}^{-/-}$mice [306]. Furthermore, in certain cases, the epitope through which the antibody was raised against was unclear. For instance, the myostatin ELISA kit from Immundiagnostik failed to indicate which region of myostatin is being detected. As a result, we strongly feel that such antibodies should be used with caution.

Recently, Schafer and colleagues developed a novel immunoplexed liquid chromatography with tandem mass spectrometry (LC-MS/MS) assay that allowed for the accurate quantification of myostatin without any cross-contamination from GDF-11 [307]. Thus, a significant improvement to this study would be to employ this technique to ascertain the levels of myostatin as the $\mathrm{Apc}^{\mathrm{Min} /+}$ mouse manifests cancer cachexia.

\subsection{The inhibition of myostatin prevented cancer cachexia in the $\mathrm{Apc}^{\mathrm{Min} / \mathrm{+}}$ mouse}

A putative role for myostatin in promoting cancer cachexia in the $\mathrm{Apc}^{\mathrm{Min} / \mathrm{+}}$ mouse came from two lines of evidence, the administration of sActRIIB and the genetic inactivation of myostatin in $A p c^{\mathrm{Min} /+}$ mice. Although the body weight of the sActRIIB treated $\mathrm{Ap} \mathrm{c}^{\mathrm{Min} / \mathrm{+}}$ mice decreased at the same rate as the dialysis buffer treated animals, the inoculation of this antagonist partially protected the skeletal muscle from atrophy and marginally improved grip strength (Figures 4.6.1-4.6.6). We were unable to observe a change in the amount of food consumed by mice treated with sActRIIB when compared to the dialysis buffer treated controls (Figure 4.6.1C). 
This result may suggest that sActRIIB may improve the skeletal muscle mass and function without altering appetite. However, this conclusion maybe too premature as we observed a significant variation in the amount of food consumed per treatment group as the trial progressed. We believe that this variability arose from both the design of the experiment and the nature of cachexia induction in the $\mathrm{Apc}^{\mathrm{Min} /+}$ mouse. First, the sensitivity of this experiment only allowed us to differentiate the food consumption rate per cage. Unfortunately, we did not have access to metabolic chambers which would have facilitated the measurement of food consumed per mouse. Second, the nature of cancer cachexia induction in the $\mathrm{Apc}^{\mathrm{Min} /+}$ mouse was sporadic. In other words, we have noticed that severely cachectic mice can co-habilitate with age matched non-cachectic mice. This would result in a heterogenous population of mice in a cage and hence alter the assessment of food consumption.

The inability of sActRIIB to fully reverse cachexia in the $A p c^{\mathrm{Min} /+}$ mouse indicated that there were factors other than myostatin which were precipitating the progressive muscle loss in this model. Unlike Zhou et al., who demonstrated the complete reversal of cachexia in the C26 model upon sActRIIB treatment, we believe that we were unable to recapitulate these findings in the $\mathrm{Apc}^{\mathrm{Min} /+}$ mouse due to the onset of anemia [63, 279]. Furthermore, the sActRIIB we have designed did not include a stabilizing moiety such as an Fc region, which could have markedly improved the half-life of the antagonist [308, 309]. Moreover, a major limitation concerning the use of sActRIIB is that this receptor is also known to interact with other TGF- $\beta$ signaling molecules such as GDF-11 and Activin A [310].

Experiments assaying the efficiency of sActRIIB should also be included. Performing immunofluorescence microscopy on the TA cryosections of sActRIIB treated $\mathrm{Apc}^{\mathrm{Min} /+}$ mice using an anti-6X His tag antibody would reveal the biodistribution of the recombinant protein. Given that this therapeutic is soluble, we would expect sActRIIB to be predominantly enriched in the extracellular space outside the myofiber. Additionally, performing a serum and skeletal muscle immunoblot for sActRIIB using an anti-6X His tag antibody would also reveal the relative amounts and the distribution of this therapeutic. Finally, sActRIIB should have been inoculated into wild type mice to determine the efficacy of this therapeutic in improving skeletal muscle mass and function during basal conditions. We would expect to see an improvement in the stated parameters as a report by Toledo et al. demonstrated that the administration of sActRIIB, though a different source to the one used in this report, profoundly increased the body weight and skeletal muscle mass of wild type mice [311]. 
To accurately discern the involvement of myostatin in manifesting cancer cachexia in the $\mathrm{Apc}^{\mathrm{Min} /+}$ mouse, we crossed $\mathrm{Apc}^{\mathrm{Min} /+}$ and $\mathrm{Mstn}^{-/-}$mice and examined the progeny for the onset of cancer cachexia and intestinal tumorigenesis. According to Figure 4.7.1, haploinsufficiency of myostatin was sufficient to delay the onset of cancer cachexia in the $\mathrm{Apc}^{\mathrm{Min} /+}$ mouse. Moreover, mice lacking myostatin demonstrated a preservation in body and skeletal muscle mass, and exhibited reduced amounts of Atrogin-1 and MuRF1 when compared to 18 week old $\mathrm{Mstn}^{+/+}:: \mathrm{ApcMin}^{/+}$mice (Figures 4.7.1-4.7.4).

\subsection{Myostatin and intestinal tumorigenesis}

Studies examining the link between myostatin and tumorigenesis are limited. A report by Professor Freyssenet's group demonstrated that Lewis-lung carcinoma (LLC) tumors ectopically grown on $\mathrm{Mstn}^{-/}$mice grow slower than on wild type controls [312]. Indeed, this delayed growth rate was in part attributed to the elevated levels of apoptosis [312]. A similar experiment performed by Benny-Klimek and colleagues, however, did not record such a dramatic reduction in the tumor size [201]. Moreover, it appeared that $\mathrm{Msth}^{-/}$mice bearing LLC and B16F10, another cachexia inducing ectopic tumor model, failed to confer any protection against cancer cachexia despite the absence of host myostatin. The reason for this disparity between two nearly identical experiments is unknown.

Nevertheless, compelling evidence for myostatin regulating tumorigenesis came from the work by Gallot and colleagues, who also demonstrated that $\mathrm{Mstn}^{-/} \because \because A p c^{\mathrm{Min} /+}$ mice exhibited a remarkable reduction in small intestinal tumor number and a longer lifespan compared to wild type $\mathrm{Mstn}^{+/+}: \because A p c^{\mathrm{Min} /+}$ mice [312]. Given that the inactivation of myostatin appears to confer a protective effect against not only cancer-induced skeletal muscle wasting but also intestinal tumorigenesis, we postulated that $\mathrm{Mstn}^{-/}$mice may exhibit a unique biology of the intestine. However, we failed to observe a difference in the intestinal length and mass of Mstn ${ }^{-/}$mice compared to $\mathrm{Mstn}^{+/+}$mice (Figure 4.11.1). Furthermore, in stark contrast to the results reported by Gallot and colleagues, we were unable to witness such a dramatic reduction in the intestinal tumor burden and tumor diameter in the $\mathrm{Mstn}^{-/}: \because A p c^{\mathrm{Min} /+}$ male mice when compared to their respective wild type controls (Figure 4.8.1) [312].

On the other hand, using the AOM/DSS model of colitis-induced carcinogenesis we have established that there was a $75 \%$ reduction in the colonic tumor burden in $\mathrm{Mstn}^{-/-}$male mice 
when compared to the wild type control (Figure 4.10.2). While this outcome appears to be very compelling, it must be stressed that this experiment should be interpreted with caution. The extent of tumorigenesis is dependent on the ability of DSS to induce inflammation in the AOM primed animal $[269,273,313]$. In other words, greater the inflammatory response, the greater incidence of colorectal tumors.

In our experiment, $\mathrm{Mstn}^{-/}$mice that consumed DSS exhibited a 5\% average decrease in body weight compared to the $15 \%$ reduction observed by $\mathrm{Mstn}^{+/+}$mice, indicating that mice devoid of myostatin are resistant to inflammation-induced loss of body weight (Figure 4.10.1). The relative resistance to weight loss due to colitis is a strong indicator of a reduced inflammatory response to DSS [269, 273, 313, 314]. Hence, the lack of inflammation in the colon conferred by the absence of myostatin might be the principle reason for the reduced incidence of colon tumors in the $\mathrm{Mstn}^{-/-}$mouse subjected to the AOM/DSS protocol. A significant improvement to this study would be to first increase the percentage of DSS to match the body weight loss exhibited by the wild type mice consuming $2.5 \%$ DSS and then subsequently quantify the colorectal tumor burden. If the weight loss matched $\mathrm{Msth}^{-/}$mice exhibited fewer colorectal tumors compared to the wild type mice consuming $2.5 \%$ DSS, we would then be able to suggest that myostatin may affect colorectal carcinogenesis. As of now, the reduced tumor burden witnessed in the $\mathrm{Mstn}^{-/-}$mice after the completion of the AOM/DSS cycle is not necessarily due to the absence of myostatin but due to the resistance of $\mathrm{Mstn}^{-/}$mice to DSS-induced inflammation.

The resilience to colitis and subsequently colitis-induced carcinogenesis exhibited by the Mstn/ mice could be due to several reasons. $\mathrm{Mstn}^{-/-}$mice are demonstrably larger than their wild type counterparts. Thus, it is unsure whether its increased body mass due to the hypertrophy and hyperplasia of the skeletal muscle can influence its vulnerability to DSS treatment. As implied previously, it is also probable that the absence of myostatin in the animal may impart an anti-inflammatory environment in the gut. Indeed, myostatin inhibition in a mouse model of chronic kidney disease (CKD) is associated with a reduction of pro-inflammatory cytokines such as IL-6, TNF- $\alpha$, and IFN- $\gamma$ [286]. There are no reports examining the relative amounts of such pro-inflammatory cytokines in the intestines of $\mathrm{Msth}^{-/}$mice when compared to their wild type controls. Thus, this study could be vastly improved by ascertaining the mRNA and protein expression levels of key inflammatory cytokines and chemokines in the intestines of mice devoid of myostatin. 
The expression of pro-inflammatory cytokines and the induction of intestinal inflammation is critically dependent on the NF- $\kappa B$ pathway [315]. Several lines of evidence have implicated the elevated activity of the NF- $\mathrm{B}$ pathway and the pathogenesis of inflammation in the guts of murine models and patients suffering from irritable bowel disease (IBD) and intestinal cancer [316-318]. Hence, to further gain insight into the relative resistance of the $\mathrm{Mstr}^{-/-}$gut to inflammation, the activation levels of certain members of the NF- $\kappa$ B pathway during basal and inflammatory conditions compared to the wild type intestines should be queried.

The differential response to AOM/DSS in the $\mathrm{Mstn}^{-/-}$mice compared to the wild type control could also be due to the nature of the intestinal cells in the $\mathrm{Mstn}^{-/-}$mice. Based on the current literature, intestinal tumors are purported to arise from the intestinal stem cells located at the base of the crypts [241, 244-247]. Measuring the depth and width of the crypts would provide an essential clue about the ISC compartment of the $\mathrm{Mstn}^{-/-}$mouse. Other experiments such as staining for cleaved caspase-3 and Ki67 positive cells in the intestines of these mice could yield information on the intestinal cell turnover in mice devoid of myostatin, as increased apoptotic activity and reduced proliferative potential is associated with the reduced incidence of intestinal tumorigenesis [319, 320].

\subsection{Murine genetic background and intestinal tumorigenesis}

The suppression in the polyp number and diameter in $A p c^{M i n /+}$ mice devoid of myostatin was recently reported by Gallot and colleagues [312]. Unlike their group, we were unable to witness such a drastic reduction in the small intestine tumor number in the $\mathrm{Msth}^{-/-} \because: A p c^{\mathrm{Min} /+}$ mice when compared to their respective wild type controls despite using a similar breeding scheme (Figure 4.8.1). This difference could be due to the use of a constitutive cre-loxP targeted $\mathrm{Msth}^{-/}$mouse maintained on a C57BL/6 background $[312,321]$. It must be noted that we did notice several differences in the wild type $\mathrm{Mstn}^{+/+}: \because A p c^{\mathrm{Min} /+}$ mice generated from the heterozygote breeding scheme (hereby referred to as $\operatorname{Mstn}^{+/+}:: A p c^{M i n /+(H e t)}$ ) when compared to our wild type $\mathrm{Mstn}^{+/+}: \because \mathrm{Apc}^{\mathrm{Min} /+}$ colony control mice.

Despite their identical genotype, the small intestine tumor multiplicity and size were vastly different between the male $\mathrm{Mstn}^{+/+}: \because A p c^{\mathrm{Min} /+}{ }^{(\mathrm{Het})}$ and the colony control $\mathrm{Mstn}^{+/+}:: A p c^{\mathrm{Min} /+}$ mice (Figure 7.13.1). Specifically, the colony control $\mathrm{Mstn}^{+/+}: \because A p c^{\mathrm{Min} /+}$ mice exhibited a higher incidence of polyps, greater average polyp diameter, and a significantly larger proportion of 
tumors greater than $2 \mathrm{~mm}$ compared to the $\mathrm{Mstn}^{+/+}:: A p c^{\mathrm{Min} /+}$ (Het) mice (Figures 7.13.1A-D). The number and the average diameter of the colon tumors in the $\mathrm{Mstn}^{+/+}: \because \mathrm{Apc}^{\mathrm{Min} /+}{ }^{\mathrm{Het})}$ mice were similar to the colony control (Figures 7.13.1E and 7.13.1F). We also noted some differences in the growth characteristics of $\mathrm{Mstn}^{+/+} \mathrm{Apc} \mathrm{Min} /+(\mathrm{Het})^{\mathrm{mice}}$ (Figures 7.13.2, 7.13.3D,

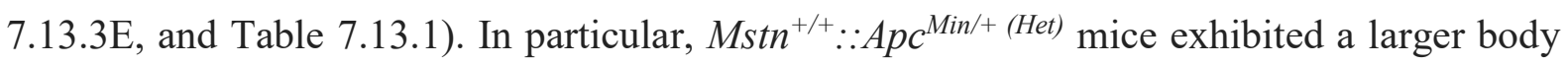
weight and greater TA, GAS, and QUAD skeletal muscle mass when compared to the age matched colony control $\mathrm{Mstn}^{+/+}: \because A p c^{\mathrm{Min} /+}$ mice (Figures 7.13.2, 7.13.3D, 7.13.3E, and Table 7.13.1). The spleen weight and tibia lengths remained unchanged (Figure 7.13.3F and Table 7.13.2).

The colony control $\mathrm{Mstn}^{+/+}$male mice wild type for the $\mathrm{Apc}$ allele $\left(\mathrm{Mstn}^{+/+}:: A p c^{+/+}\right)$also exhibited some differences to the $\mathrm{Mstn}^{+/+}: \because \mathrm{Apc}^{+/+}$mice generated by the heterozygote breeding scheme, referred to as $\mathrm{Mstn}^{+/+}: \because A p c^{+/+}{ }^{(H e t)}$. The $\mathrm{Mstn}^{+/+}:: A p c^{+/+}$(Het) GAS, QUAD, spleen weights, and tibia lengths were significantly greater than the colony control $\mathrm{Mstn}^{+/+}:: A p c^{+/+}$ mice (Figures 7.13.3A-C, Tables 7.13.3 and 7.13.4). Furthermore, the intestinal lengths of the colony control $\mathrm{Mstn}^{+/+}: \because \mathrm{Apc}^{+/+}$mice were significantly shorter than the $\mathrm{Mstn}^{+/+}: \because \mathrm{Apc}^{+/+}(\mathrm{Het})$ mice (Figures 7.13.4A and 7.13.4D). In addition, $\mathrm{Mstn}^{+/+}: \because A p c^{+/+(H e t)}$ mice displayed a significantly heavier uncleared and cleared intestinal weights compared to the $\mathrm{Mstn}^{+/+}:: \mathrm{Apc}^{+/+}$ colony control mice (Figures 7.13.4B, 7.13.4C, 7.13.4E, and 7.13.4F).

Despite these differences, it must be stressed that $\mathrm{Mstn}^{+/+}: \because A p c^{\mathrm{Min} /+}(\mathrm{Het})$ and $\mathrm{Mstn}^{+/+}: \because A p c^{+/+}$ (Het) mice were still significantly smaller in both body mass and skeletal muscle mass when compared to the $\mathrm{Mstn}^{-/}: \because A p c^{\mathrm{Min} /+}$ and $\mathrm{Mstn}^{-/}: \because A p c^{+/+}$mice that were generated from the same heterozygote breeding scheme (Figures 7.13.5A, 7.13.5B, 7.13.6A, and Table 7.13.6). Moreover, these $\mathrm{Mstn}^{+/+}: \because A p c^{\mathrm{Min} /+(H e t)}$ mice possessed larger spleens compared to the $\mathrm{Msth}^{-/-}$ $\because A p c^{\mathrm{Min} /+}$ mice, indicating a greater extent of splenomegaly and anemia (Figure 7.13.6B). However, given the striking differences between the colony control and the $\mathrm{Mstn}^{+/+}: \because \mathrm{Apc} \mathrm{Min} /+^{-}$ (Het) mice, we felt that it was not advisable to assess the tumor burden of the colony control $\mathrm{Mstn}^{+/+} \because: A p c^{\mathrm{Min} /+}$ mice and compare it to the $\mathrm{Mstn}^{+/-} \because \mathrm{Apc}^{\mathrm{Min} /+}$ and $\mathrm{Mstn}^{-/-} \because \mathrm{Apc}^{\mathrm{Min} /+}$ mice generated from the heterozygote breeding scheme (Figure 7.13.7).

The reasons for this variation in the growth and tumor characteristics of the $\mathrm{Mstn}^{+/+}: \because \mathrm{Apc} \mathrm{Min} /+^{\mathrm{H}}$ mice generated from the heterozygote breeding pair compared to the wild type $\mathrm{Mstn}^{+/+} \because: A p c^{\mathrm{Min} /+}$ colony controls is unknown. One such possibility would be that the Mstn ${ }^{-/-}$ 
mice used to first generate the heterozygotes may not be identical to the $A p c^{M i n /+}$ mouse as previously thought. $\mathrm{Mstn}^{-/-}$mice used in this report were generated by first disrupting the Mstn gene by homologous targeting in embryonic stem cells and then crossing the pups to C57BL/6 females [140]. Conversely, the $A p c^{M i n /+}$ mouse was first generated by $N$-ethyl-N-nitrosourea (ENU) mutagenesis in founder C57BL/6 mice then maintained on a C57BL/6 background [255]. Although these two mice originate from the same genetic background, our data suggests that the $\mathrm{Mstn}^{+/+}: \because A p c^{\mathrm{Min} /+}$ mice from the heterozygote breeding scheme may exhibit genetic diversity over several loci when compared to the colony control $\mathrm{Mstn}^{+/+}: \because A p c^{\mathrm{Min} /+}$ mice. This consideration is particularly important as polyposis in the $A p c^{\mathrm{Min} /+}$ mice is susceptible to genetic modifiers [265]. Although such modifiers of the $A p c^{M i n /+}$ phenotype are prevalent between in-bred mouse strains, there have been reports of $A p c^{\mathrm{Min} /+}$ modifiers occurring within the same substrain [266]. Thus, to determine whether the $\mathrm{Mstn}^{-/-}$and $A p c^{\mathrm{Min} /+}$ mice in our colony are of the same substrain, a background characterization panel offered by Charles River should be conducted.

\subsection{Future experiments to investigate the link between myostatin and cancer}

This study would have benefitted from the execution of in vitro experiments designed to investigate the molecular mechanism of how myostatin may influence cancer. Specifically, the proliferation and apoptosis rates of cancer cell lines from numerous tissue sources should be examined in the presence of biologically active myostatin (Figure 5.6.1). These in vitro experiments should be repeated in primary cancer cell lines such as the organoids collected from the $A p c^{\mathrm{Min} /+}$ mice (Figure 5.6.1). If there is an alteration in the proliferation and death kinetics of the cancer cell in response to myostatin, the molecular mechanisms that initiated such a change can be examined by gene expression and immunoblot analysis for bona fide targets of myostatin, such as phosphorylated Smad2/3 levels [150].

If there are no effects on the growth rates of cancer cells treated with myostatin, then we could surmise that there may be an indirect mechanism through which myostatin regulates tumorigenesis. The absence of myostatin leads to a profound change in not only the skeletal muscle, but also in the adipose tissue, bone, and overall body metabolism [284, 322]. These physiological changes have been linked to the circulation of muscle-specific hormones other than myostatin $[2,24,323]$. To gain an understanding on how the absence of myostatin can influence the growth of tumors, additional in vitro experiments using both primary and 
secondary cancer cell lines cultured in the conditioned media of primary skeletal muscle cells isolated from $\mathrm{Mstn}^{-/}$animals should be performed. This particular experiment would provide an indication if there is another muscle derived hormone unique to $\mathrm{Mstn}^{-/}$animals which may impart a restrictive environment for cancer growth (Figure 5.6.1).

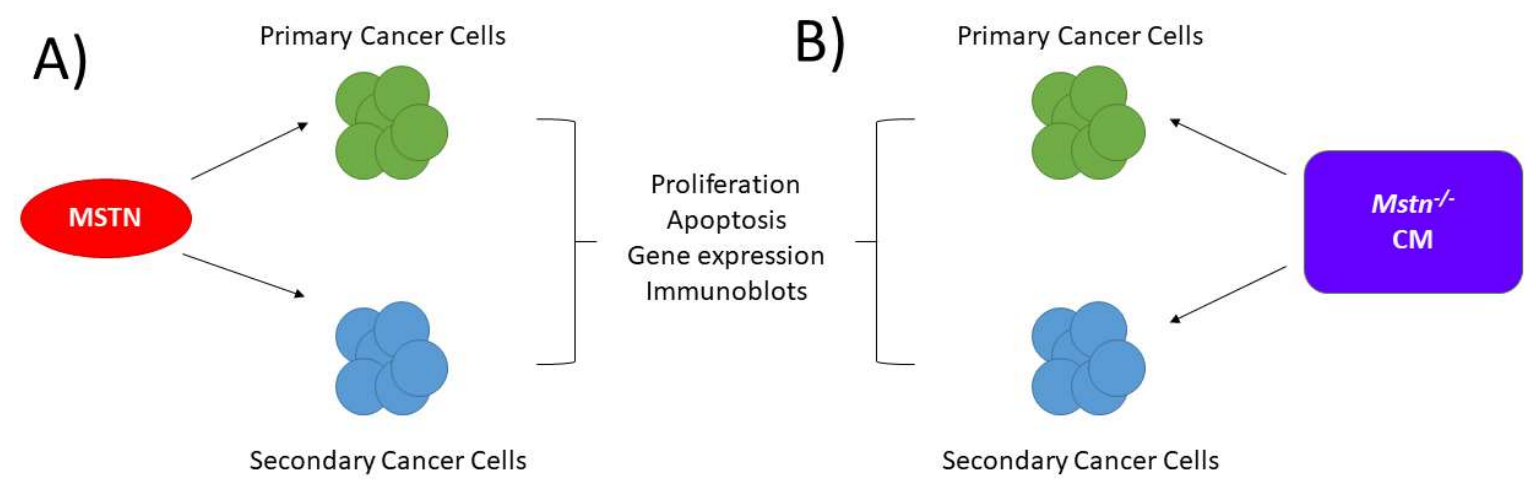

Figure 5.6.1: Investigating the molecular mechanism of myostatin and cancer.

A schematic diagram to highlight the in vitro experiments that could be performed in the future to discern the molecular mechanism through which myostatin can regulate tumorigenesis. A) Schematic diagram representing the treatment of primary and secondary cancer cell lines with biologically active myostatin. B) Schematic diagram representing the treatment of primary and secondary cancer cell lines with the conditioned media (CM) from $\mathrm{Mstn}^{-/}$primary muscle cell cultures. Both experiments would require the assessment of the growth kinetics. Provided that there is an alteration in the growth rates of the cancer cells, subsequent experiments will be performed to identify the signaling components that are affected by the treatment.

Inconclusive results from the above in vitro experiments would necessitate the execution of several more pre-clinical models of cancer to study the impact of myostatin and tumorigenesis. For instance, the suppression of myostatin through CRISPR/Cas9 in other clinically relevant mouse models of cancer such as the Rip1Tag2 transgenic model for pancreatic cancer could confirm whether this muscle specific hormone could affect the growth rates of cancers from other tissues [324-326]. In a report published by Wei and colleagues, the researchers demonstrated that the muscle specific deletion of myostatin using the Staphylococcus aureus (SaCas9)/adeno-associated virus 8 (AAV8) system 9 with a tissue-specific double muscle creatine kinase $(\mathrm{dMCK})$ promoter expression preserved muscle mass and function in mice undergoing LLC induced cancer cachexia [327]. It would be possible to employ such a strategy on cachectic $A p c^{M i n /+}$ mice to investigate whether the direct silencing of myostatin in skeletal muscle is sufficient to delay the onset of cancer cachexia and influence intestinal tumorigenesis. At the same time, the question of whether the over-expression of myostatin could modulate the incidence and growth of $\mathrm{Apc}^{\mathrm{Min} /+}$ small intestinal tumors could be addressed by the delivery of myostatin cDNA through AAVs. 



\section{Conclusions}

It is becoming apparent that the function of myostatin is not just limited to regulating the size of the skeletal muscle [150]. Indeed, since the discovery of transgenic $\mathrm{Mstn}^{-/-}$mice, several reports have demonstrated that myostatin can regulate the physiology of the adipose tissue depots [140, 280, 328]. Recently, myostatin has been shown to directly influence the skeletal system. In fact, a report by Pap and colleagues have uncovered that myostatin induces osteoclastogenesis and stimulates bone destruction in murine models of rheumatoid arthritis [329]. The link between myostatin and a relatively distant organ, the intestine, is currently unexplored.

This thesis presents several lines of data to support the notion that myostatin regulates the loss of skeletal muscle mass during colorectal cancer-induced cachexia. First, we have demonstrated that myostatin is expressed during cancer cachexia in the $\mathrm{Apc}^{\mathrm{Min} / \mathrm{+}}$ mouse. Evidence for the augmented activity of myostatin was highlighted by the partial rescue of skeletal muscle size and function by sActRIIB inoculation in cachectic $\mathrm{Apc}^{\mathrm{Min} /+}$ mice. Furthermore, the genetic ablation of myostatin in $\mathrm{Apc}^{\mathrm{Min} /+}$ mice delayed the onset of cancer cachexia. It is currently unclear whether myostatin directly regulates colorectal carcinogenesis. We have demonstrated that there is no reduction in the number of tumors in the intestines of $A p c^{M i n /+}$ mice devoid of myostatin. While there was a significant decrease in the average small intestinal tumor size, the magnitude of this reduction observed in the $\mathrm{Mstn}^{-/}: \because A p c^{\mathrm{Min} /+}$ mice when compared to the wild type control was marginal (15.33\%). Using the AOM/DSS model of colorectal carcinogenesis, however, we have shown that $M s t n^{-/}$mice are strikingly resistant to colitis-induced colorectal tumors. Though highly encouraging, it is currently unclear whether the reduction in the colorectal tumor burden exhibited by the $\mathrm{Msth}^{-/-}$mouse was a direct result of myostatin or due to the attenuated inflammatory response to the colitis-inducing agent. Hence, given the disparity in the results garnered from the $A p c^{M i n /+}$ and AOM/DSS models of colorectal cancer, it is currently too early to declare myostatin as a novel pro-tumorigenic factor. 


\section{$7 \quad$ Appendices}

7.1 $A p c^{M i n /+}$ mice demonstrated cancer cachexia

Table 7.1.1 (Section 4.1, Figure 4.1.1): Body weights for male $A \mathrm{Ac}^{\mathrm{Min} /+}$ mice $(\mathrm{n}=9-50)$ and wild type controls $(\mathrm{n}=11-38)$ measured from 5 to 20 weeks.

Wild Type

\begin{tabular}{|c|c|c|c|c|c|c|c|c|c|c|c|c|c|c|c|c|}
\hline Age (weeks) & 5 & 6 & 7 & 8 & 9 & 10 & 11 & 12 & 13 & 14 & 15 & 16 & 17 & 18 & 19 & 20 \\
\hline Average weight (g) & 18.92 & 20.03 & 21.24 & 22.34 & 23.11 & 24.04 & 24.75 & 25.41 & 25.87 & 26.30 & 26.68 & 27.29 & 27.44 & 27.84 & 27.97 & 28.56 \\
\hline \pm S.D. & 1.69 & 1.76 & 1.58 & 1.75 & 1.67 & 1.69 & 1.75 & 1.60 & 1.65 & 1.62 & 1.77 & 1.63 & 1.54 & 1.53 & 1.44 & 1.51 \\
\hline $\mathrm{N}$ & 12 & 28 & 31 & 31 & 31 & 38 & 38 & 38 & 37 & 38 & 38 & 38 & 38 & 35 & 18 & 11 \\
\hline Maximum & 20.75 & 23.55 & 24.63 & 26.34 & 26.84 & 28.32 & 28.38 & 29.36 & 29.20 & 30.54 & 31.19 & 31.74 & 31.96 & 31.68 & 31.08 & 31.28 \\
\hline Minimum weight $(\mathrm{g})$ & 15.18 & 16.20 & 17.90 & 18.70 & 19.00 & 20.40 & 20.73 & 21.70 & 22.30 & 22.60 & 22.07 & 23.00 & 24.60 & 25.04 & 25.22 & 25.65 \\
\hline
\end{tabular}

$$
A p c^{\operatorname{Min} /+}
$$

\begin{tabular}{|c|c|c|c|c|c|c|c|c|c|c|c|c|c|c|c|c|}
\hline \multirow[b]{2}{*}{ Age (weeks) } & & & & & & & & & & & & & & & & \\
\hline & 5 & 6 & 7 & 8 & 9 & 10 & 11 & 12 & 13 & 14 & 15 & 16 & 17 & 18 & 19 & 20 \\
\hline Average weight (g) & 18.72 & 19.39 & 20.61 & 21.49 & 22.31 & 22.97 & 23.51 & 24.11 & 24.62 & 24.83 & 24.85 & 24.97 & 24.55 & 24.49 & 23.92 & 24.95 \\
\hline \pm S.D. & 1.30 & 1.62 & 1.35 & 1.65 & 1.58 & 1.76 & 1.65 & 1.48 & 1.49 & 1.46 & 1.67 & 1.91 & 2.17 & 2.29 & 2.45 & 2.43 \\
\hline $\mathrm{N}$ & 18 & 30 & 32 & 35 & 41 & 40 & 41 & 47 & 47 & 50 & 50 & 49 & 46 & 27 & 14 & 9 \\
\hline Maximum weight $(\mathrm{g})$ & 22.22 & 23.49 & 23.99 & 25.42 & 26.58 & 27.17 & 26.84 & 27.79 & 28.36 & 27.95 & 28.35 & 28.43 & 28.50 & 28.06 & 28.90 & 29.19 \\
\hline Minimum weight (g) & 17.08 & 16.76 & 18.48 & 18.88 & 19.46 & 19.79 & 20.30 & 21.56 & 21.37 & 21.69 & 19.46 & 19.70 & 17.90 & 19.27 & 21.03 & 21.94 \\
\hline
\end{tabular}


Table 7.1.2 (Section 4.1, Table 4.1.1): Male $\mathrm{Apc}^{\mathrm{Min} / \mathrm{+}}$ mice used for the cancer cachexia stratification trial.

\begin{tabular}{|c|c|c|c|c|c|c|}
\hline Genotype & ID & $\begin{array}{l}\text { Peak body weight } \\
\text { (g) }\end{array}$ & $\begin{array}{l}\text { Live body weight at } \\
\text { termination }(\mathrm{g})\end{array}$ & $\begin{array}{l}\text { Percentage } \\
\text { Change }\end{array}$ & Stratification & $\begin{array}{c}\text { Age at } \\
\text { Termination }\end{array}$ \\
\hline ApcMin/+ & 412 & 24.56 & 24.15 & 1.7 & Non-cachectic & $17 \mathrm{w} 1 \mathrm{~d}$ \\
\hline ApcMin/+ & $910(1 / 5)$ & 26.7 & 26.1 & 2.2 & Non-cachectic & $17 \mathrm{w} 1 \mathrm{~d}$ \\
\hline ApcMin/+ & 950 & N/A & 26.07 & N/A & Non-cachectic & $13 w 6 d$ \\
\hline ApcMin/+ & 949 & N/A & 26.21 & N/A & Non-cachectic & $13 w 6 d$ \\
\hline ApcMin/+ & 965 & $\mathrm{~N} / \mathrm{A}$ & 25.93 & N/A & Non-cachectic & $13 \mathrm{w} 5 \mathrm{~d}$ \\
\hline ApcMin/+ & 967 & N/A & 28.01 & N/A & Non-cachectic & $13 w 5 d$ \\
\hline ApcMin/+ & 968 & N/A & 26.8 & N/A & Non-cachectic & $13 \mathrm{w} 5 \mathrm{~d}$ \\
\hline ApcMin/+ & 416 & 25.6 & 24.37 & 4.8 & Early & $17 \mathrm{w} 2 \mathrm{~d}$ \\
\hline ApcMin/+ & $901(3 / 5)$ & 26.53 & 24.61 & 7.2 & Early & $17 \mathrm{w} 5 \mathrm{~d}$ \\
\hline ApcMin/+ & $906(1 / 3)$ & 25.98 & 24.45 & 5.9 & Early & $17 w 6 d$ \\
\hline ApcMin/+ & $908(2 / 3)$ & 26.6 & 24.86 & 6.5 & Early & $17 \mathrm{w} 2 \mathrm{~d}$ \\
\hline ApcMin/+ & $907(3 / 3)$ & 26.24 & 24.77 & 5.6 & Early & $17 \mathrm{w} 0 \mathrm{~d}$ \\
\hline ApcMin/+ & 414 & 26.6 & 22.88 & 14.0 & Intermediate & $17 \mathrm{w} 4 \mathrm{~d}$ \\
\hline ApcMin/+ & $905(4 / 5)$ & 25.39 & 22.89 & 9.8 & Intermediate & $17 \mathrm{w} 0 \mathrm{~d}$ \\
\hline ApcMin/+ & $903(5 / 5)$ & 27.42 & 24.14 & 12.0 & Intermediate & $16 \mathrm{w} 6 \mathrm{~d}$ \\
\hline ApcMin/+ & $904(6 / 5)$ & 25.16 & 22.3 & 11.4 & Intermediate & $17 \mathrm{w} 1 \mathrm{~d}$ \\
\hline ApcMin/+ & 413 & 26.58 & 21.15 & 20.4 & Severe & $17 \mathrm{w} 0 \mathrm{~d}$ \\
\hline ApcMin/+ & 417 & 24.57 & 19.46 & 20.8 & Severe & $15 \mathrm{w} 0 \mathrm{~d}$ \\
\hline ApcMin/+ & 299 & 23.7 & 18.72 & 21.0 & Severe & $17 \mathrm{w} 0 \mathrm{~d}$ \\
\hline ApcMin/+ & 21 & 23 & 17.97 & 21.9 & Severe & $17 w 4 d$ \\
\hline ApcMin/+ & 415 & 27.17 & 21.18 & 22.0 & Severe & $19 \mathrm{w} 0 \mathrm{~d}$ \\
\hline ApcMin/+ & 902 & 27.51 & 20.41 & 25.8 & Severe & $21 \mathrm{w} 6 \mathrm{~d}$ \\
\hline
\end{tabular}

Please note, mice ID in brackets represent the old numbering system used for the mouse. 
Table 7.1.3 (Section 4.1, Table 4.1.1): Tibia lengths $(\mathrm{mm})$ for wild type $(\mathrm{n}=6)$, noncachectic $(n=7)$, early $(n=5)$, intermediate $(n=4)$, and severe $(n=6)$ cachectic $^{\mathrm{Apc}^{\mathrm{Min} /+}}$ mice.

\begin{tabular}{lccccc} 
& & \multicolumn{4}{c}{$A p c^{\text {Min } /+}$} \\
\cline { 2 - 5 } & Wild Type & Non-Cachectic & Early & Intermediate & Severe \\
\hline Tibia Length $(\mathrm{mm})$ & 16.84 & 16.86 & 16.80 & 16.81 & 16.88 \\
\pm S.D. & 0.09 & 0.28 & 0.03 & 0.03 & 0.20 \\
\hline
\end{tabular}

Significance was assessed by one-way ANOVA followed by Tukey's post-hoc test: No significant difference was observed. 


\subsection{Cachectic $\mathrm{Apc}^{\mathrm{Min} /+}$ mice displayed skeletal muscle and adipose tissue atrophy}

Table 7.2.1 (Section 4.2, Figure 4.2.1): Skeletal muscle weights of $A p c^{\mathrm{Min} /+}$ mice stratified by percentage body weight loss.

\begin{tabular}{lccccc} 
& & \multicolumn{4}{c}{$A p c^{\mathrm{Min} /+}$} \\
\cline { 3 - 6 } & Wild Type & $\begin{array}{c}\text { Non- } \\
\text { Cachectic }\end{array}$ & Early & Intermediate & Severe \\
\hline TA weight $(\mathrm{g})$ & 0.0471 & 0.0488 & 0.0494 & 0.0448 & $0.0393 \dagger$ \\
\pm S.D. & 0.0021 & 0.0030 & 0.0037 & 0.0061 & 0.0092 \\
\hline EDL weight $(\mathrm{g})$ & 0.0094 & 0.0105 & 0.0099 & $0.0079 \dagger$ & $0.0065 \ddagger$ \\
\pm S.D. & 0.0009 & 0.0017 & 0.0011 & 0.0014 & 0.0010 \\
\hline GAS weight $(\mathrm{g})$ & 0.1469 & 0.1527 & 0.1444 & $0.1343 \dagger$ & $0.1154 \dagger$ \\
\pm S.D. & 0.0051 & 0.0086 & 0.0020 & 0.0070 & 0.0170 \\
\hline SOL weight $(\mathrm{g})$ & 0.0083 & 0.0085 & 0.0070 & 0.0074 & 0.0069 \\
\pm S.D. & 0.0004 & 0.0007 & 0.0014 & 0.0013 & 0.0013 \\
\hline QUAD weight $(\mathrm{g})$ & 0.2083 & 0.2183 & 0.1989 & $0.1754 \dagger$ & $0.1494 \dagger$ \\
\pm S.D. & 0.0111 & 0.0164 & 0.0040 & 0.0137 & 0.0174 \\
\hline
\end{tabular}

$\uparrow$ Significantly different to non-cachectic group $(\mathrm{p}<0.05)$

\$ Significantly different to non-cachectic group $(\mathrm{p}<0.01)$

Significance was assessed by one-way ANOVA followed by Tukey's post-hoc test 
7.3 Skeletal muscle atrophy in cachectic $\mathrm{Apc}^{\mathrm{Min} / \mathrm{+}}$ mice was associated with the increased expression of the ubiquitin-proteasome pathway

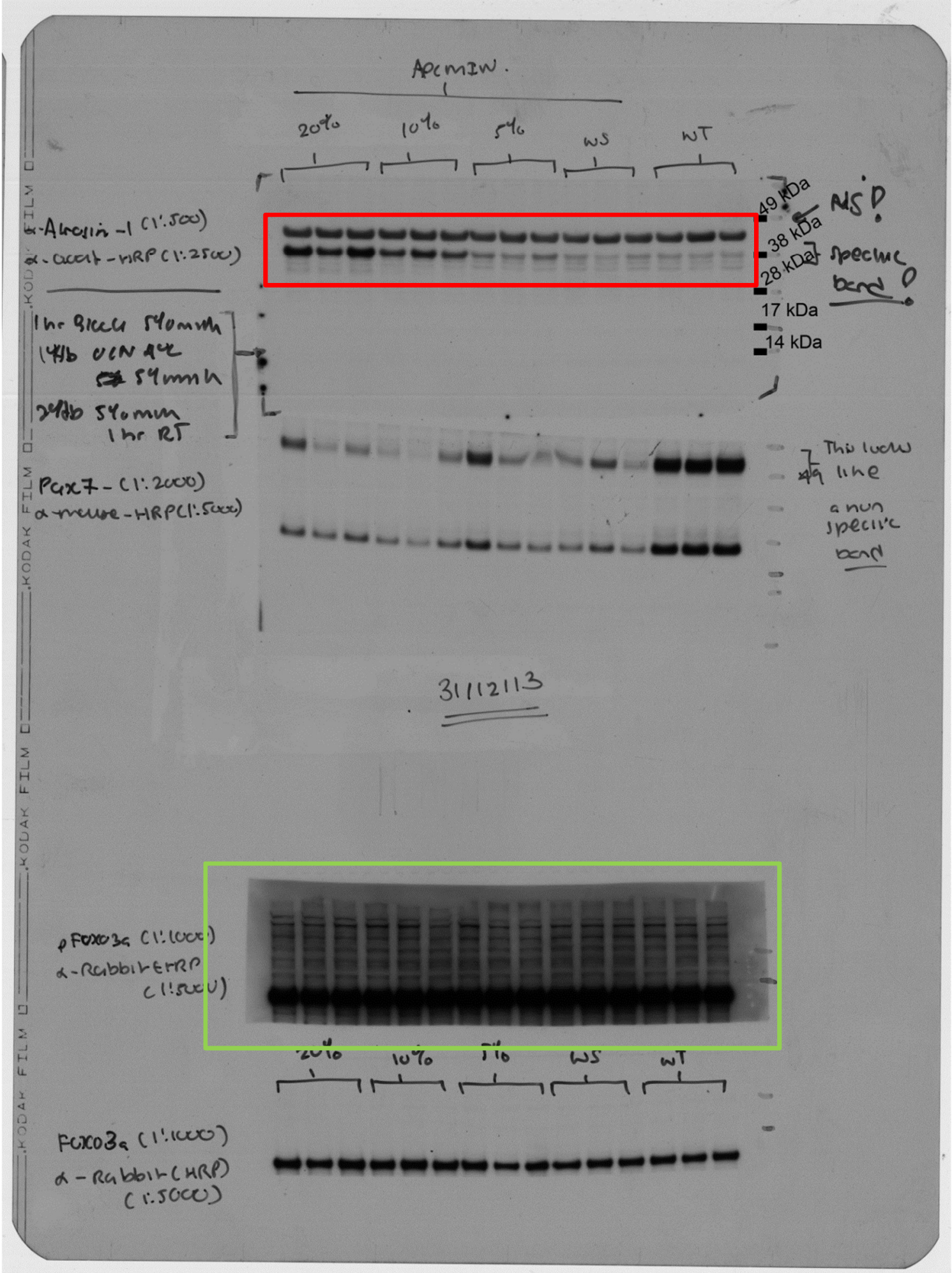

Figure 7.3.1 (Section 4.3, Figure 4.3.2A and 4.3.2B): Immunoblot for Atrogin-1.

The red box highlights the region used for image preparation. Please note that there was a nonspecific band above the specific $38 \mathrm{kDa}$ Atrogin-1 band. Please note that the cropped image was reflected along the y-axis. Molecular weight markers are recorded on the image. Furthermore, the top half of this immunoblot was cut to probe for p-FoxO3a (highlighted in a green box, Figure 7.3.2). 


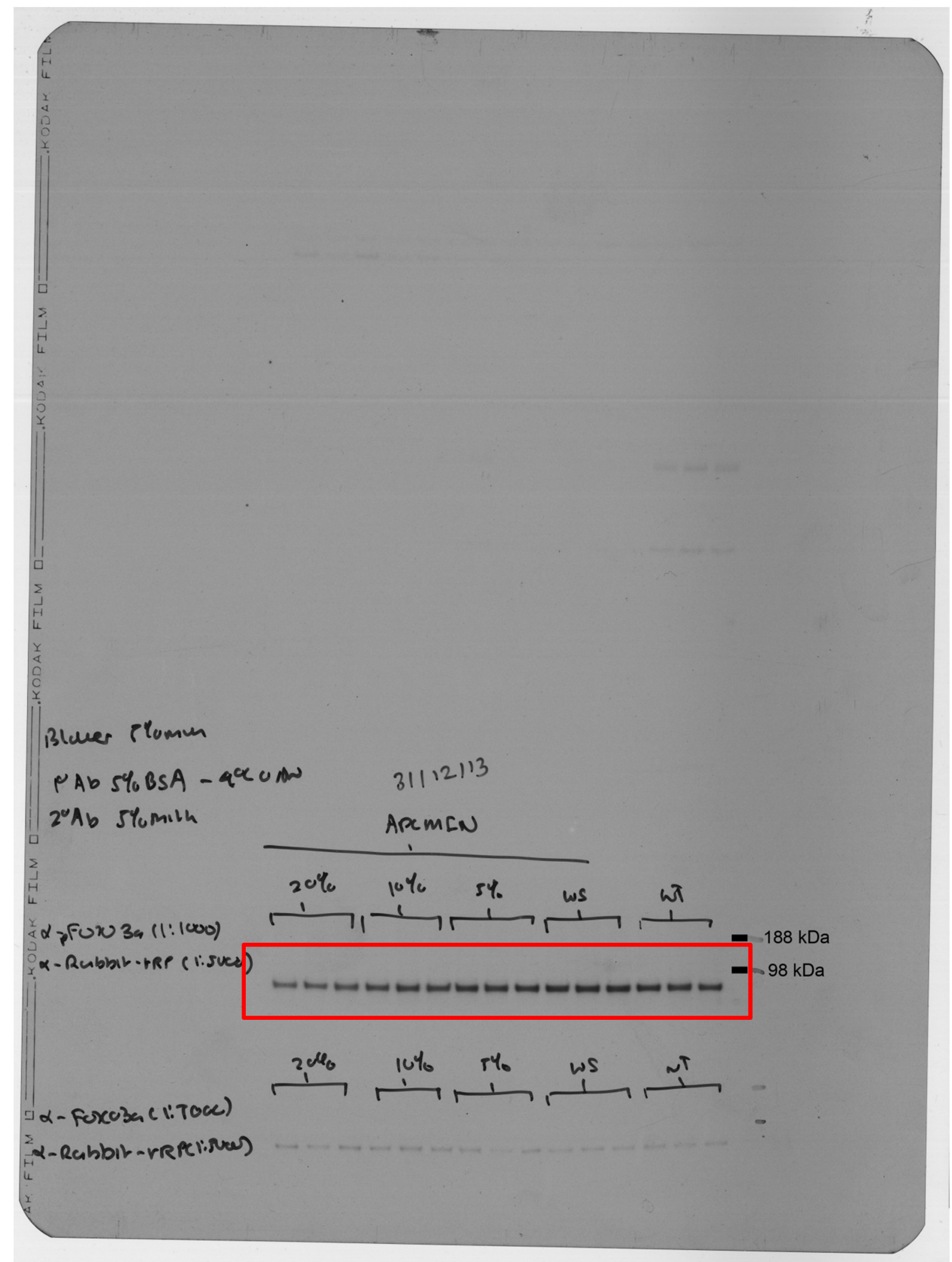

Figure 7.3.2 (Section 4.3, Figures 4.3.2A and 4.3.2B): Immunoblot for p-FoxO3a.

The red box highlights the region used for image preparation. Please note that the cropped image was reflected along the y-axis. Molecular weight markers are recorded on the image. The bottom half of this immunoblot was used to probe for Atrogin-1 (Figure 7.3.1). 


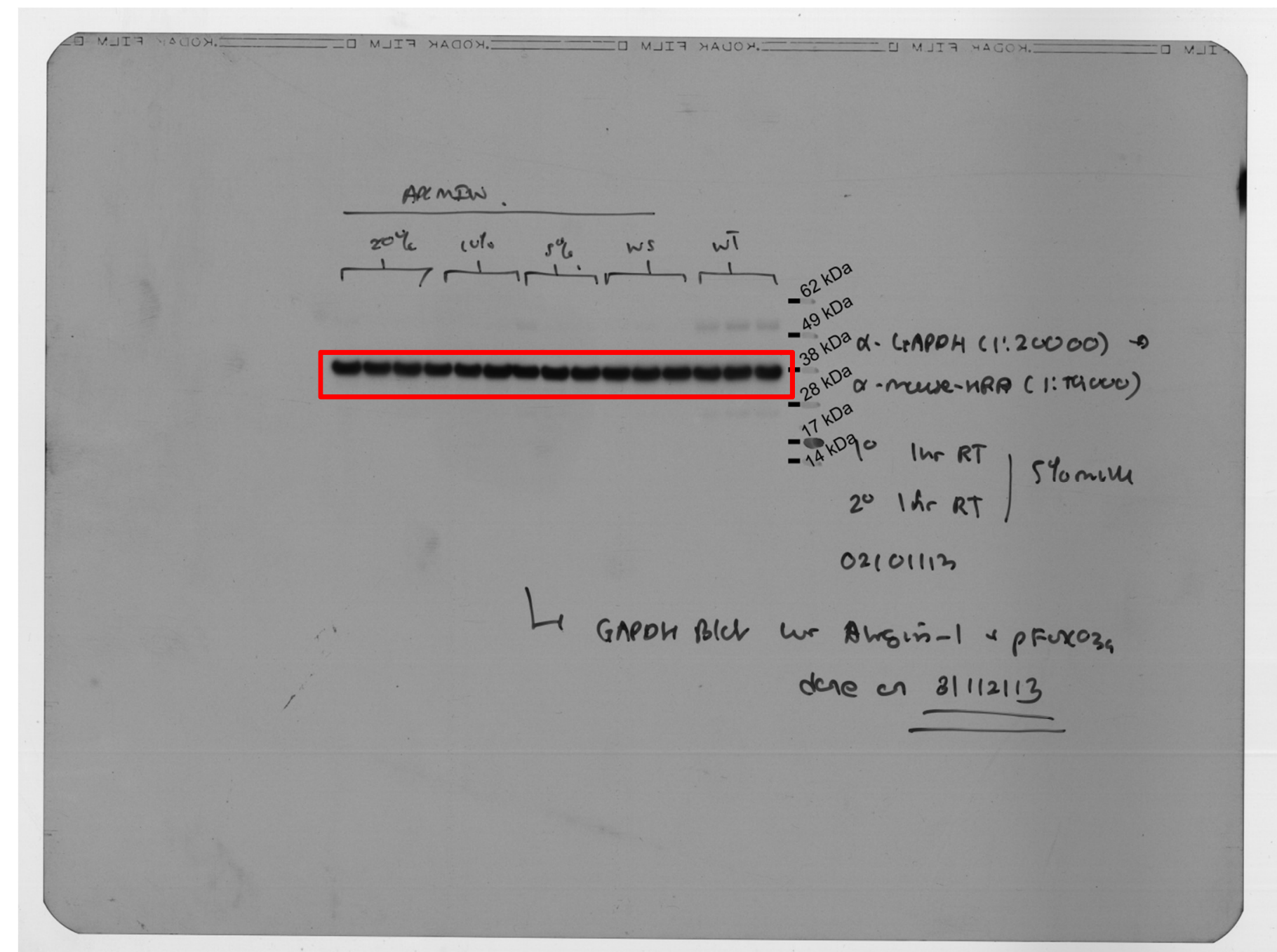

Figure 7.3.3 (Section 4.3): Immunoblot for GAPDH corresponding to Atrogin-1 and p-FoxO3a immunoblots represented in Figure 7.3.1 and Figure 7.3.2 (Section 4.3, Figure 4.3.2A and 4.3.2B).

The red box highlights the region used for image preparation. Please note that the cropped image was reflected along the y-axis. Molecular weight markers are recorded on the image. Please note that the top half of this immunoblot was used to detect for p-FoxO3a (Figure 7.3.2). 


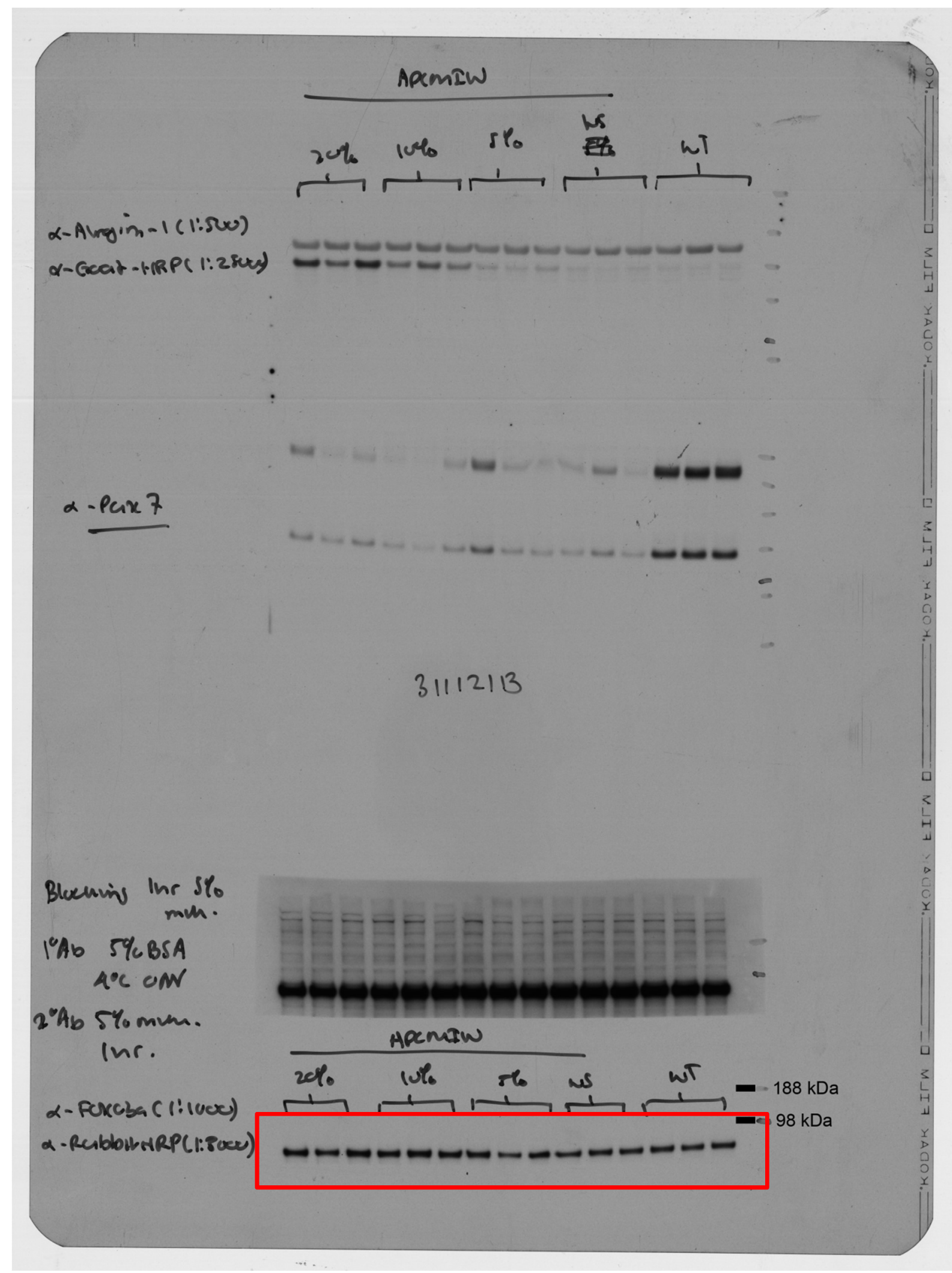

Figure 7.3.4 (Section 4.3, Figures 4.3.2A and 4.3.2B): Immunoblot for FoxO3a immunoblots.

The red box highlights the region used for image preparation. Please note that the cropped image was reflected along the y-axis. Furthermore, the top half of this immunoblot (above $62 \mathrm{kDa}$ ) was used to probe for FoxO3a. The bottom half of the immunoblot (at $62 \mathrm{kDa}$ ) was used to probe for GAPDH (see Figure 7.3.5). Molecular weight markers are recorded on the image. 


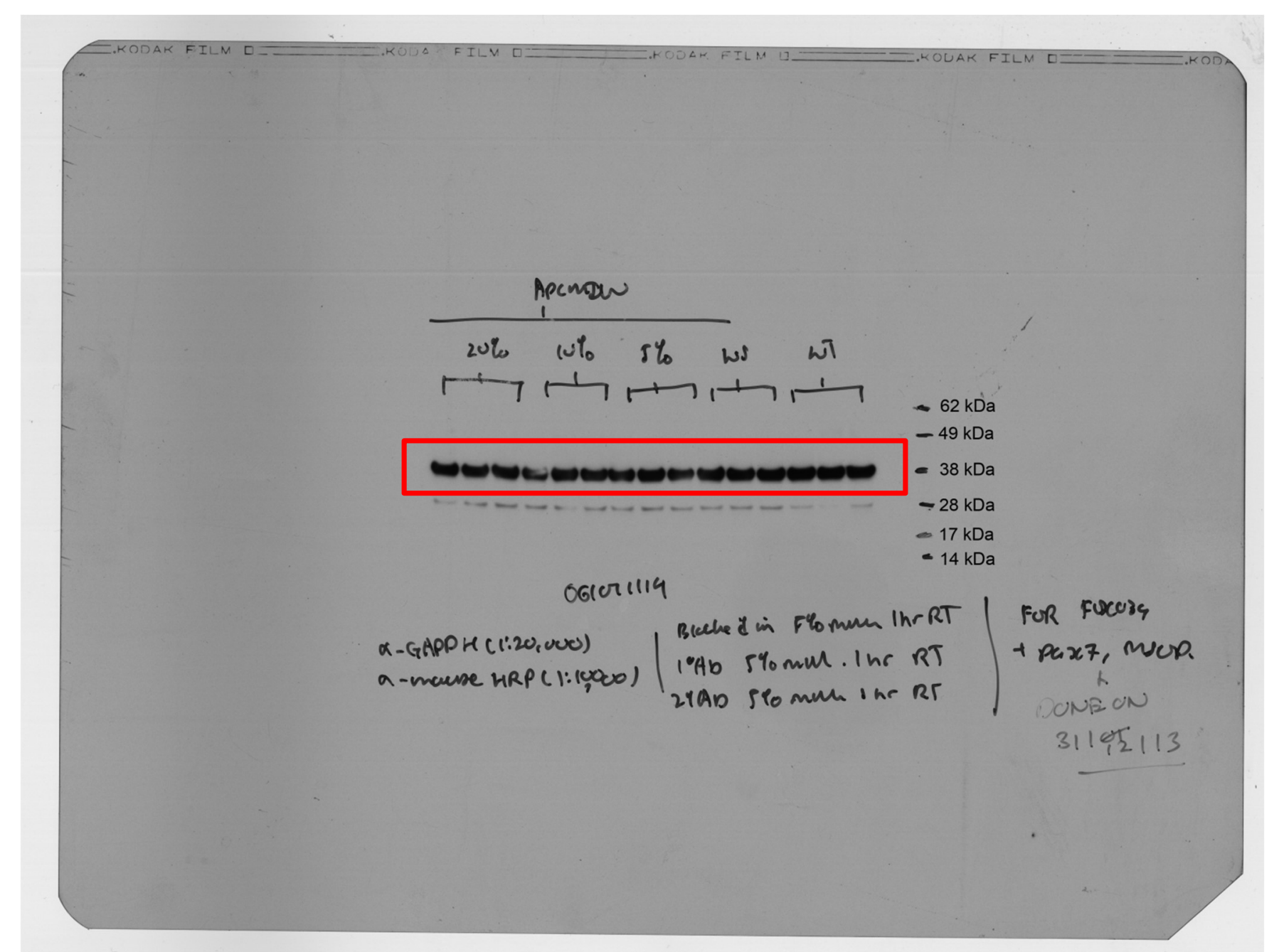

Figure 7.3.5: Immunoblot for GAPDH corresponding to FoxO3a immunoblots represented in Figure 7.3.4 (Section 4.3, Figure 4.3.2A and 4.3.2B).

The red box highlights the region used for densitometry calculation. The top half of this immunoblot (above $62 \mathrm{kDa}$ ) was used to probe for FoxO3a (Figure 7.3.4). Molecular weight markers are recorded on the image. 


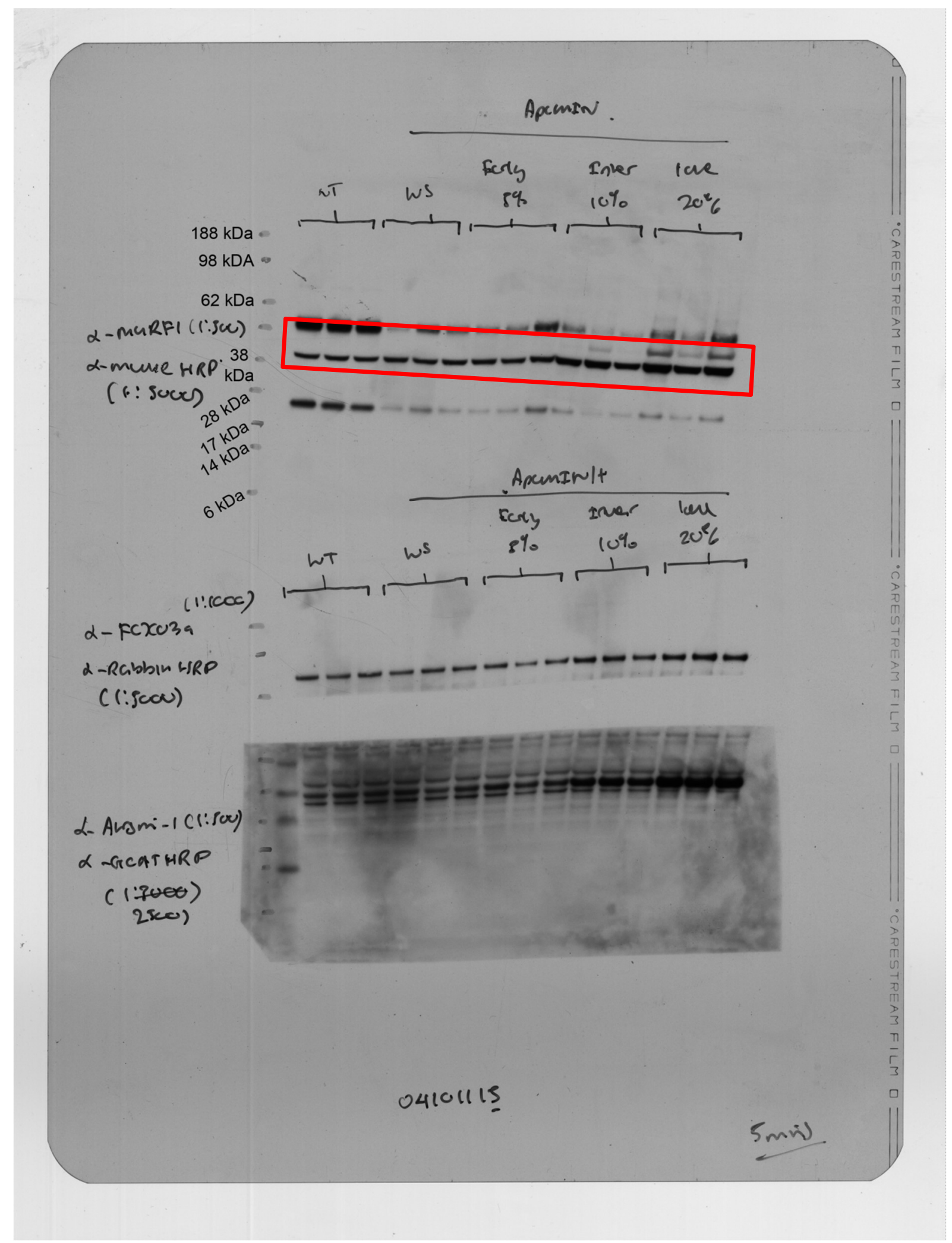

\section{Figure 7.3.6 (Section 4.3, Figures 4.3.2A and 4.3.2B): Immunoblot for MuRF1.}

The red box highlights the region used for image preparation. The cropped image was slighted tilted to capture all the MuRF1 bands. Molecular weight markers are recorded on the image, except for the $49 \mathrm{kDa}$ band (located between the $62 \mathrm{kDa}$ and $38 \mathrm{kDa}$ bands). The $49 \mathrm{kDa}$ molecular weight marker was not recorded on the image as the text would have occluded notes that are written on the immunoblot. Please note that this blot was incubated with anti-MuRF1 for 2 days at $4^{\circ} \mathrm{C}$. 


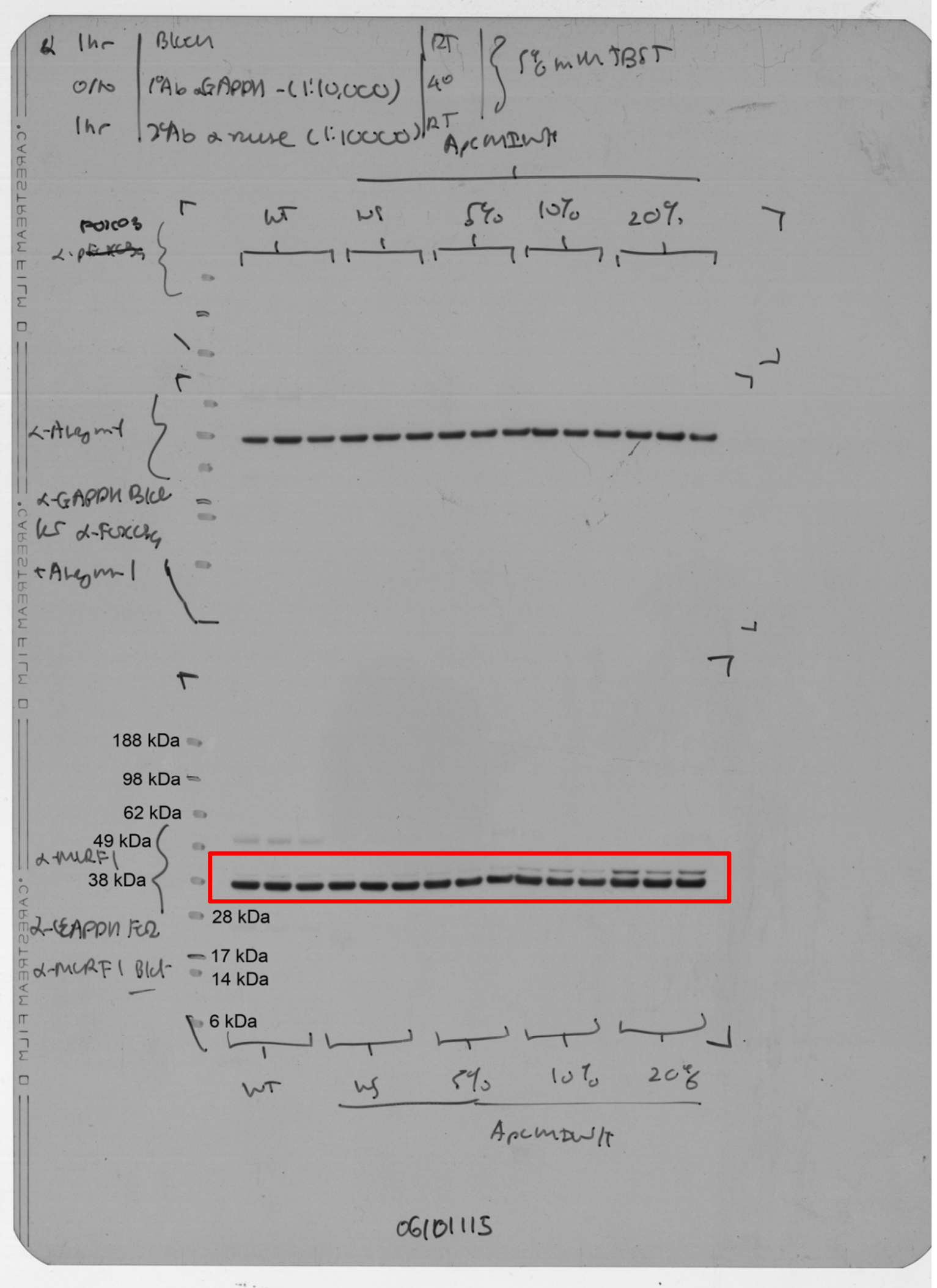

Figure 7.3.7: Immunoblot for GAPDH corresponding to MuRF1 immunoblots represented in Figure 7.3.6 (Section 4.3, Figures 4.3.2A and 4.3.2B).

The red box highlights the region used for densitometry calculation. Molecular weight markers are recorded on the image. Please note that this blot was incubated with anti-GAPDH for 2 days at $4^{\circ} \mathrm{C}$. 
7.4 Myostatin gene expression was elevated in the skeletal muscles of cachectic $\mathrm{Apc}^{\mathrm{Min} / \mathrm{H}}$ mice

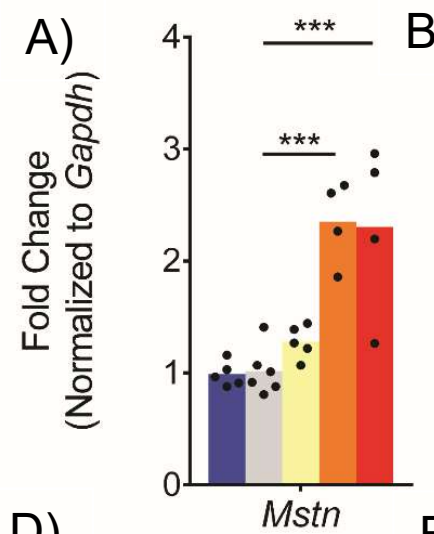

D)

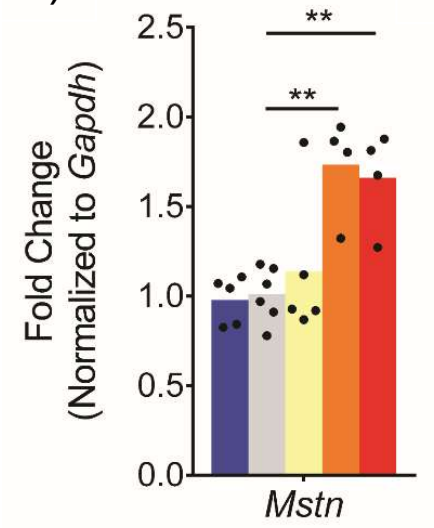

B)

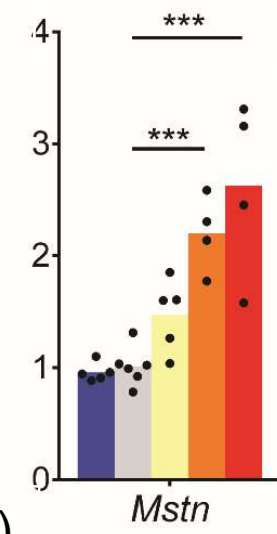

E)

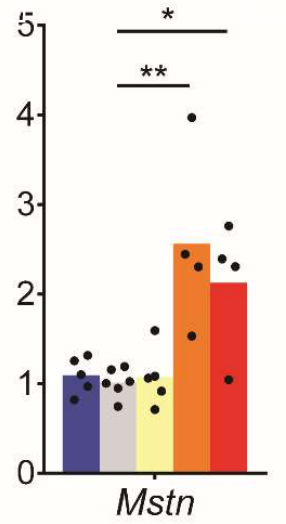

C)

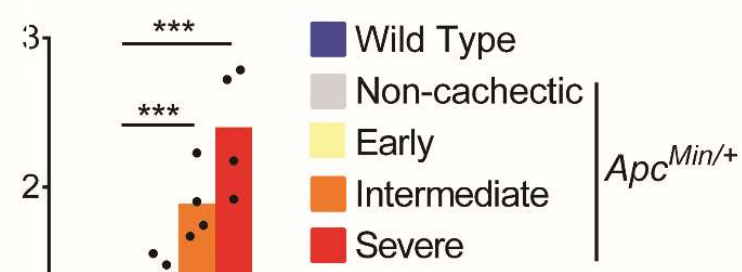

F)

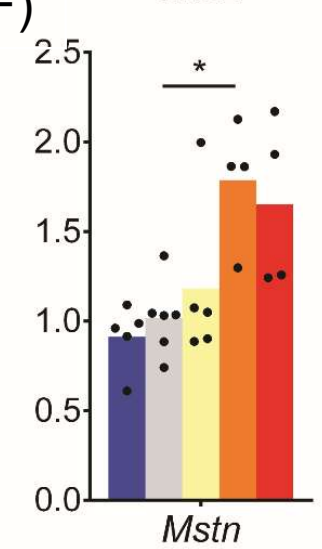

Figure 7.4.1 (Section 4.4, Figure 4.4.1): Myostatin gene expression levels were elevated in the GAS muscles of moderately to severely cachectic $\mathrm{Apc}^{\mathrm{Min} /+}$ mice.

Relative gene expression of myostatin (Mstn) in the GAS muscles of wild type $(n=5)$, noncachectic $(n=6)$, early $(n=5)$, intermediate $(n=4)$, and severely $(n=4)$ cachectic $\operatorname{Apc}^{\text {Min } /+}$ mice. Gene expression was normalized to Gapdh. (A-F) Each bar graph represents one RT-qPCR replicate. Figure 7.4.1A was represented in Figure 4.4.1. Significance was assessed by one-way ANOVA followed by Tukey's post-hoc test: ${ }^{*} \mathrm{p}<0.05,{ }^{* *} \mathrm{p}<0.01,{ }^{* * *} \mathrm{p}<0.001 . \Delta \mathrm{Ct}$ was used to calculate significance. 
A)

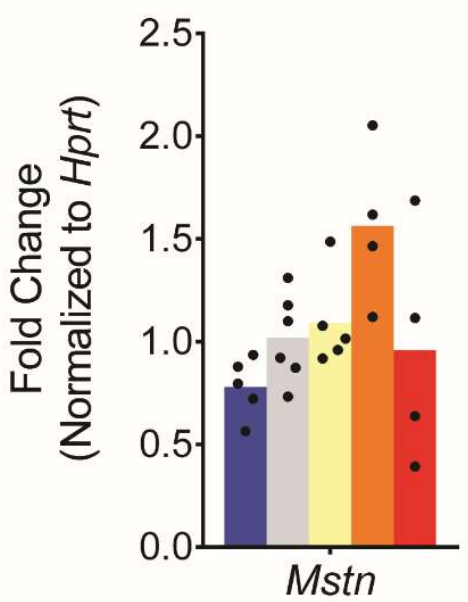

B)

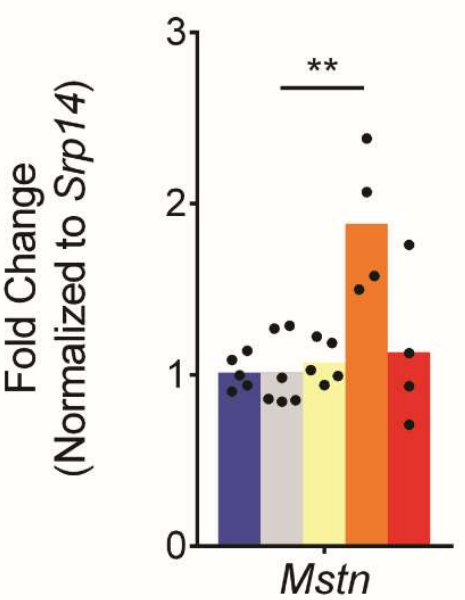

Wild Type

Non-cachectic

Early

\begin{tabular}{l|l} 
Intermediate & $A p c^{\mathrm{Min} /+}$ \\
Severe &
\end{tabular}

Figure 7.4.2 (Section 4.4, Figure 4.4.1): Myostatin gene expression levels in the GAS muscles of the cachectic $\mathrm{Apc}^{\mathrm{Min} / \mathrm{+}}$ mice.

Relative gene expression of myostatin (Mstn) in the GAS muscles of wild type $(\mathrm{n}=5)$, noncachectic $(\mathrm{n}=6)$, early $(\mathrm{n}=5)$, intermediate $(\mathrm{n}=4)$, and severely $(\mathrm{n}=4)$ cachectic $^{\mathrm{Apc}} \mathrm{Min} / \mathrm{+}^{\mathrm{mice}}$. Gene expression was normalized to A) Hprt and B) Srp 14. Significance was assessed by one-way ANOVA followed by Tukey's post-hoc test: $* \mathrm{p}<0.05, * * \mathrm{p}<0.01, * * * \mathrm{p}<0.001 . \Delta \mathrm{Ct}$ was used to calculate significance. 

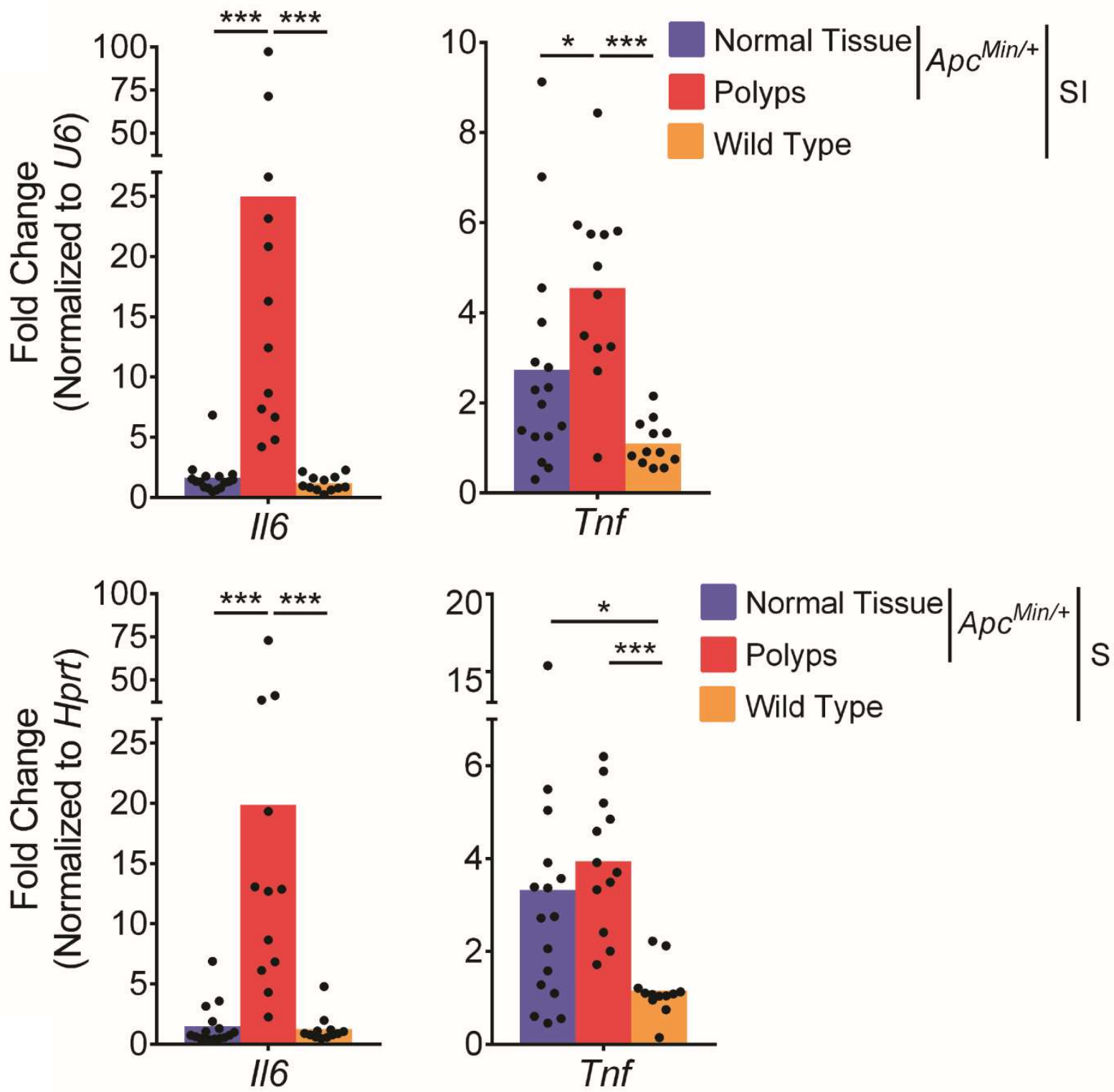

Figure 7.5.1 (Section 4.5, Figure 4.5.1): Small intestine polyps from $\mathrm{Apc}^{\mathrm{Min} /+}$ male mice express interleukin-6 and TNFa.

Relative gene expression of interleukin-6 (Il6) and TNF $\alpha(T N F)$, variant 1 in the normal noncancerous small intestinal tissue (normal tissue) $(n=4)$ and polyps $(n=3)$ of 17 to 19 week old $A p c^{M i n /+}$ mice. Small intestine (n=3) from 17 to 19 week old wild type mice were used as controls. Gene expression was normalized to either U6 or Hprt. Significance was assessed by one-way ANOVA followed by Tukey's post-hoc test: ${ }^{*} \mathrm{p}<0.05,{ }^{* *} \mathrm{p}<0.01,{ }^{* * *} \mathrm{p}<0.001 . \Delta \mathrm{Ct}$ was used to calculate significance. $\mathrm{N}$ represents the number of mice used for this experiment. However, with respect to the data collected for the normal non-cancerous small intestinal tissue, wild type small intestine, and polyps, it must be noted that the small intestine was divided into 4 equal parts per mouse. Thus, each data point represents the fold change for a particular region of the small intestine. 

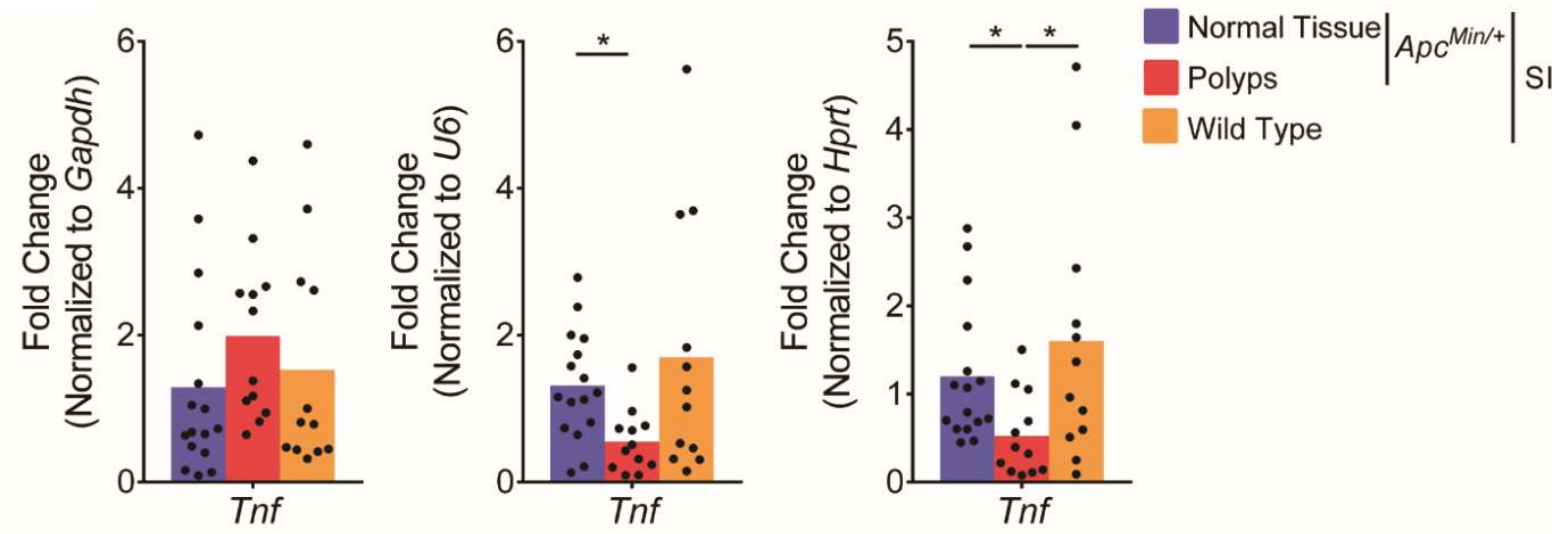

Figure 7.5.2 (Section 4.5, Figure 4.5.1): Small intestine polyps from $A p c^{M i n /+}$ male mice do not express TNF $\alpha$ variant 2.

Relative gene expression of TNF $\alpha(T N F)$, variant 2 in the normal non-cancerous small intestinal tissue (normal tissue) $(\mathrm{n}=4)$ and polyps $(\mathrm{n}=3)$ of 17 to 19 week old $A p c^{\mathrm{Min} /+}$ mice. Small intestine $(\mathrm{n}=3)$ from 17 to 19 week old wild type mice were used as controls. Gene expression was normalized to either Gapdh, U6, or Hprt. Significance was assessed by one-way ANOVA followed by Tukey's post-hoc test: ${ }^{*} \mathrm{p}<0.05, * * \mathrm{p}<0.01, * * * \mathrm{p}<0.001 . \Delta \mathrm{Ct}$ was used to calculate significance. $\mathrm{N}$ represents the number of mice used for this experiment. However, with respect to the data collected for the normal non-cancerous small intestinal tissue, wild type small intestine, and polyps, it must be noted that the small intestine was divided into 4 equal parts per mouse. Thus, each data point represents the fold change for a particular region of the small intestine. 
7.6 Soluble ActRIIB administration partially halted the loss of skeletal muscle mass and marginally improved grip strength in cachectic $\mathrm{Apc}^{\mathrm{Min} /+}$ mice

Table 7.6.1 (Section 4.6, Figures 4.6.1-4.6.3): Dosage of sActRIIB administered to $\mathrm{Apc}^{\mathrm{Min} /+}$ mice at 16 weeks.

Injection Number

\begin{tabular}{lcccccccccccc}
\hline & 1 & 2 & 3 & 4 & 5 & 6 & 7 & 8 & 9 & 10 & 11 & 12 \\
\hline sActRIIB $(\mu \mathrm{g} /$ gram body weight) & 5.0 & $5.0 \dagger$ & 3.0 & 3.0 & 3.5 & 3.5 & 3.5 & 3.5 & 4.5 & 4.5 & 4.5 & 4.5 \\
\hline$\dagger 3$ out 10 mice were administered $2.5 \mu \mathrm{g} /$ gram body weight of sActRIIB. The remaining mice received \\
$5.0 \mu \mathrm{g} /$ gram body weight of sActRIIB.
\end{tabular}


Table 7.6.2 (Section 4.6, Figure 4.6.2A): Grip strength, measured in Newtons (N), for age-matched wild type $(\mathrm{n}=7)$, dialysis buffer $(\mathrm{n}=11)$, and sActRIIB treated $\mathrm{Apc}^{\mathrm{Min} /+}$ $(\mathrm{n}=9)$ mice.

\begin{tabular}{lccc} 
& & \multicolumn{2}{c}{$A p c^{\mathrm{Min} /+}$} \\
\cline { 2 - 4 } & Wild Type & Dialysis Buffer & sActRIIB \\
\hline Grip Strength Trial 1 (N) & 1.020 & $0.736 \dagger$ & 0.799 \\
\pm S.D. & 0.113 & 0.259 & 0.053 \\
\cline { 2 - 4 } Grip Strength Trial 2 (N) & 0.784 & $0.459 \dagger$ & $0.691 \dagger \dagger$ \\
\pm S.D. & 0.108 & 0.089 & 0.061 \\
\cline { 2 - 4 } Grip Strength Trial 3 (N) & 0.711 & $0.520 \dagger$ & $0.620 \dagger$ \\
\pm S.D. & 0.0385 & 0.106 & 0.027 \\
\hline
\end{tabular}

$\uparrow$ Significantly different to wild type age-matched mice $(\mathrm{p}<0.05)$

$\$$ Significantly different to dialysis buffer treatment $(\mathrm{p}<0.05)$

Significance was assessed by one-way ANOVA followed by Tukey's post-hoc test 
A)
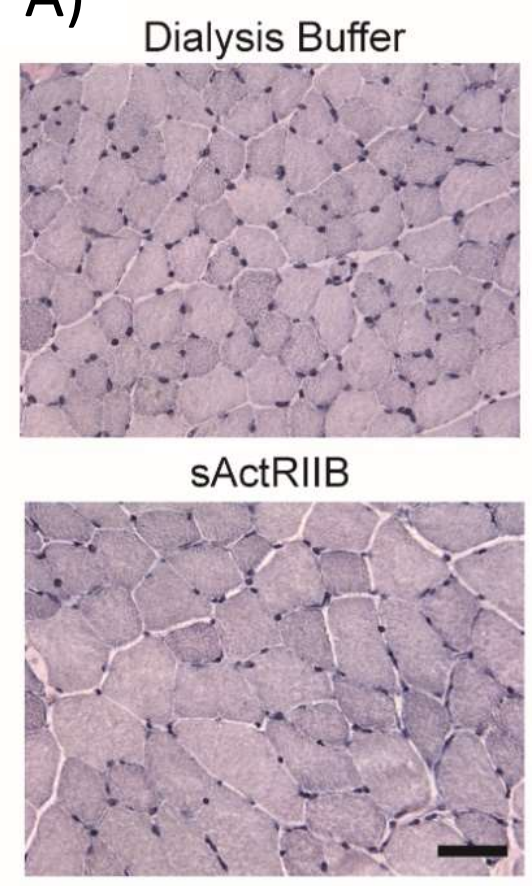

B)

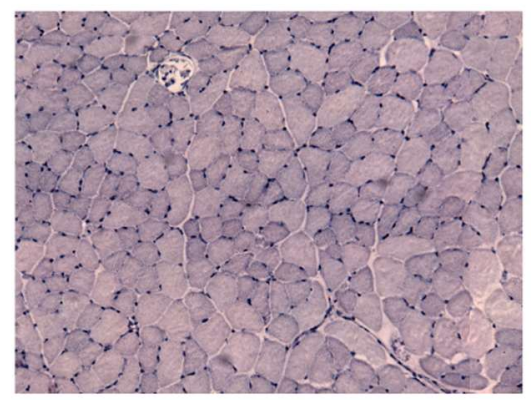

Dialysis Buffer
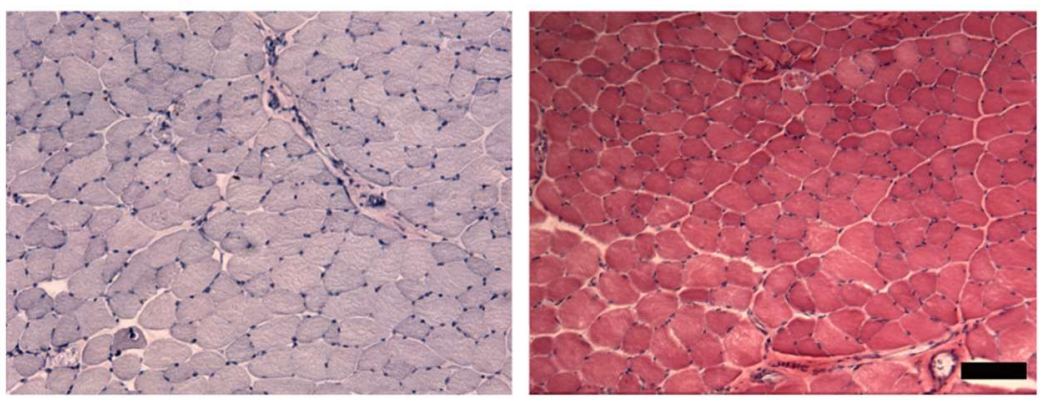

SActRIIB
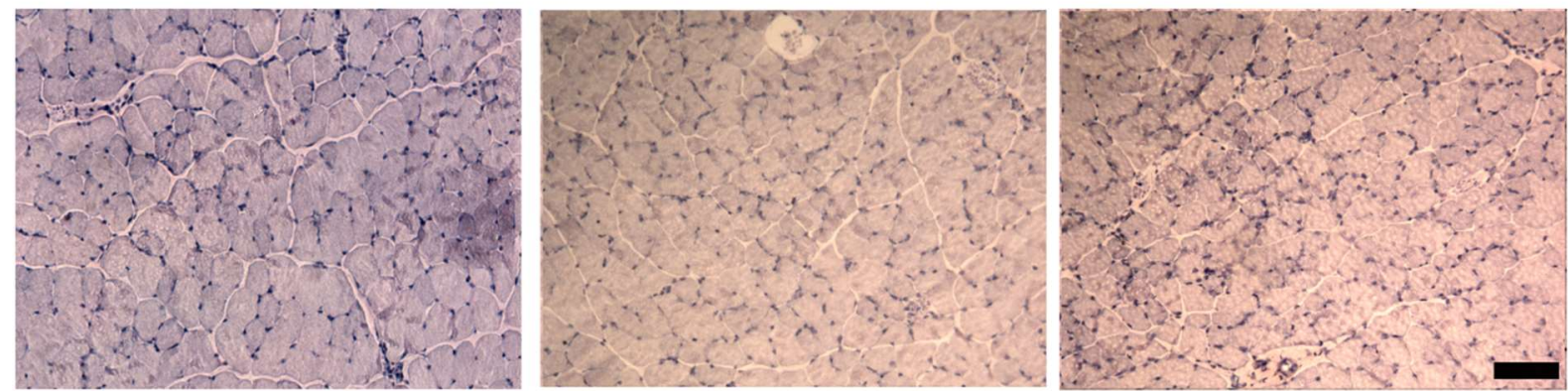

Figure 7.6.1 (Section 4.6, Figure 4.6.3): Soluble ActRIIB treatment of male $\mathrm{Apc}^{\mathrm{Min} /+}$ mice at 16 weeks failed to improve the TA myofiber cross-sectional area.

A) Representative H\&E images of cryosectioned TA. Scale bar represents $50 \mu \mathrm{m}$.

B) Additional representative H\&E images of cryosectioned TA. Scale bar represents $100 \mu \mathrm{m}$. Images are taken from the tiled images of the TA's used for the assessment of the myofiber cross-sectional area. Please note that only the brightness and contrast were adjusted. 
Table 7.6.3 (Section 4.6, Figure 4.6.2B): Skeletal muscle weights for dialysis buffer $(\mathrm{n}=11)$ and sActRIIB treated $\mathrm{Apc}^{\mathrm{Min} /+}(\mathrm{n}=9)$ mice.

$A p c^{\mathrm{Min} /+}$

\begin{tabular}{lcc} 
& Dialysis Buffer & sActRIIB \\
\hline TA weight $(\mathrm{g})$ & 0.0319 & 0.0319 \\
\pm S.D. & 0.0078 & 0.0048 \\
\hline EDL weight $(\mathrm{g})$ & 0.0071 & 0.0075 \\
\pm S.D. & 0.0019 & 0.0013 \\
\hline GAS weight $(\mathrm{g})$ & 0.0932 & 0.0989 \\
\pm S.D. & 0.0307 & 0.0189 \\
\hline SOL weight $(\mathrm{g})$ & 0.0077 & 0.0073 \\
\pm S.D. & 0.0009 & 0.0018 \\
\hline QUAD weight $(\mathrm{g})$ & 0.1315 & 0.1353 \\
\pm S.D. & 0.0393 & 0.0267 \\
\hline
\end{tabular}

Please note, this table of results represents the data collected for all the mice used for this trial. No omissions were made. Significance was assessed by Student's t-test with Welch correction. No significant difference was observed. 
Table 7.6.4 (Section 4.6, Figure 4.6.2B): Skeletal muscle weights for dialysis buffer $(n=8)$ and sActRIIB treated $\mathrm{Apc}^{\mathrm{Min} /+}(\mathrm{n}=9)$ mice.

$$
\mathrm{Apc}^{\mathrm{Min} /+}
$$

\begin{tabular}{lcc} 
& Dialysis Buffer & sActRIIB \\
\hline TA weight $(\mathrm{g})$ & 0.0275 & $0.0319 \dagger$ \\
\pm S.D. & 0.0027 & 0.0048 \\
\hline EDL weight $(\mathrm{g})$ & 0.0064 & 0.0075 \\
\pm S.D. & 0.0014 & 0.0013 \\
\hline GAS weight $(\mathrm{g})$ & 0.0755 & $0.0989 \dagger$ \\
\pm S.D. & 0.0047 & 0.0189 \\
\hline SOL weight $(\mathrm{g})$ & 0.0073 & 0.0073 \\
\pm S.D. & 0.0006 & 0.0018 \\
\hline QUAD weight $(\mathrm{g})$ & 0.1093 & $0.1353 \dagger$ \\
\pm S.D. & 0.0101 & 0.0267 \\
\hline
\end{tabular}

$\uparrow$ Significantly different to dialysis buffer treated controls $(\mathrm{p}<0.05)$ $\$$ Significantly different to dialysis buffer treated controls $(p<0.01)$

Please note that three mice were omitted from the dialysis buffer treated group (Mice ID 969, 971, 973).

Significance was assessed by Student's t-test with Welch correction. 
Table 7.6.5 (Section 4.6, Figures 4.6.1-4.6.3): Tibia length $(\mathrm{mm})$ for dialysis buffer $(\mathrm{n}=11)$ and sActRIIB treated $\mathrm{Apc}^{\mathrm{Min} /+}(\mathrm{n}=9)$ mice.

\begin{tabular}{lcc} 
& \multicolumn{2}{c}{$A p c^{\mathrm{Min} /+}$} \\
\cline { 2 - 3 } & Dialysis Buffer & sActRIIB \\
\hline Tibia Length (mm) & 17.05 & 17.06 \\
\pm S.D. & 0.10 & 0.11 \\
\hline
\end{tabular}

Significance was assessed by Student's t-test with Welch correction. No significant difference was observed. 
Table 7.6.6 (Section 4.6, Figures 4.6.4 and 4.6.5): Dosage of sActRIIB administered to $\mathrm{Apc}^{\mathrm{Min} /+}$ mice at 14 weeks.

Injection Number

\begin{tabular}{lccccccccccccc} 
& 1 & 2 & 3 & 4 & 5 & 6 & 7 & 8 & 9 & 10 & 11 & 12 & 13 \\
\hline $\begin{array}{l}\text { sActRIIB }(\mu \mathrm{g} / \text { gram } \\
\text { body weight })\end{array}$ & 3.0 & 3.0 & 3.0 & 3.5 & 3.5 & 4.5 & 4.5 & 4.5 & 4.5 & 4.5 & 4.5 & 4.5 & 4.5 \\
\hline
\end{tabular}


Table 7.6.7 (Section 4.6, Figure 4.6.5B): Skeletal muscle weights for dialysis buffer $(n=6)$ and sActRIIB treated $\mathrm{Apc}^{\mathrm{Min} /+}(\mathrm{n}=5)$ mice.

\begin{tabular}{lcc} 
& \multicolumn{2}{c}{$A^{\mathrm{Min} /+}$} \\
\cline { 2 - 3 } & Dialysis Buffer & sActRIIB \\
\hline TA weight $(\mathrm{g})$ & 0.0308 & 0.0329 \\
\pm S.D. & 0.0045 & 0.0037 \\
\hline EDL weight $(\mathrm{g})$ & 0.0075 & 0.0066 \\
\pm S.D. & 0.0020 & 0.0013 \\
\hline GAS weight $(\mathrm{g})$ & 0.0880 & 0.0939 \\
\pm S.D. & 0.0200 & 0.0163 \\
\hline SOL weight $(\mathrm{g})$ & 0.0070 & 0.0076 \\
\pm S.D. & 0.0008 & 0.0006 \\
\hline QUAD weight $(\mathrm{g})$ & 0.1302 & 0.1387 \\
\pm S.D. & 0.0207 & 0.0245 \\
\hline
\end{tabular}

Significance was assessed by Student's t-test with Welch correction. No significant difference was observed. 
Table 7.6.8 (Section 4.6, Figures 4.6.4 and 4.6.5): Tibia length $(\mathrm{mm})$ for dialysis buffer $(\mathrm{n}=6)$ and sActRIIB treated $\mathrm{Apc}^{\mathrm{Min} /+}(\mathrm{n}=5)$ mice.

\begin{tabular}{lcc} 
& \multicolumn{2}{c}{$A p c^{\mathrm{Min} /+}$} \\
\cline { 2 - 3 } & Dialysis Buffer & sActRIIB \\
\hline Tibia Length $(\mathrm{mm})$ & 17.05 & 17.06 \\
\pm S.D. & 0.10 & 0.11 \\
\hline
\end{tabular}

Significance was assessed by Student's t-test with Welch correction. No significant difference was observed. 
7.7 The genetic ablation of myostatin prevented the onset of cancer cachexia in $\mathrm{Apc}^{\mathrm{Min} /+}$ mice

Table 7.7.1 (Section 4.7, Figure 4.7.1C): Skeletal muscle weights for $\mathrm{Mstn}^{+/+}:: \mathrm{Apc}^{\mathrm{Min} /+}(\mathrm{n}=6)$, $\mathrm{Mstn}^{+/-:}: \mathrm{Apc}^{\mathrm{Min} /+}(\mathrm{n}=4)$, and $\mathrm{Mstn}^{-/}: \because \mathrm{Apc}^{\mathrm{Min} /+}(\mathrm{n}=10)$ mice and their respective wild type controls, $\mathrm{Mstn}^{+/+}:: \mathrm{Apc}^{+/+}(\mathrm{n}=6), \mathrm{Mstn}^{+/-}: \because \mathrm{Apc}^{+/+}(\mathrm{n}=6)$, and $\mathrm{Mstn}^{-/}: \because \mathrm{Apc}^{+/+}(\mathrm{n}=11) \mathrm{mice}$.

\begin{tabular}{|c|c|c|c|c|c|c|}
\hline & \multicolumn{2}{|c|}{$\mathrm{Mstn}^{+/+}$} & \multicolumn{2}{|c|}{$\mathrm{Mstn}^{+/-}$} & \multicolumn{2}{|c|}{ Msth $^{-/-}$} \\
\hline & $A p c^{+/+}$ & $A p c^{\mathrm{Min} /+}$ & $A p c^{+/+}$ & $A p c^{\mathrm{Min} /+}$ & $A p c^{+/+}$ & $A p c^{\operatorname{Min} /+}$ \\
\hline TA weight (g) & 0.0476 & $0.0357 \$$ & 0.0621 & $0.0567 \$$ & 0.0875 & 0.0822 \\
\hline \pm S.D. & 0.0029 & 0.0066 & 0.0024 & 0.0016 & 0.0114 & 0.0054 \\
\hline EDL weight (g) & 0.0101 & 0.0081 & 0.0237 & 0.0126 & 0.0194 & 0.0191 \\
\hline \pm S.D. & 0.0018 & 0.0025 & 0.0260 & 0.0009 & 0.0045 & 0.0026 \\
\hline GAS weight (g) & 0.1517 & $0.1123 \dagger$ & 0.2045 & $0.1855 \ddagger$ & 0.3171 & 0.2999 \\
\hline \pm S.D. & 0.0066 & 0.0258 & 0.0089 & 0.0049 & 0.0240 & 0.0287 \\
\hline SOL weight (g) & 0.0074 & 0.0078 & 0.0243 & 0.0079 & 0.0108 & 0.0105 \\
\hline \pm S.D. & 0.0008 & 0.0006 & 0.0258 & 0.0008 & 0.0013 & 0.0016 \\
\hline $\begin{array}{l}\text { QUAD weight } \\
\text { (g) }\end{array}$ & 0.225 & $0.175 t$ & 0.328 & $0.291 \ddagger$ & 0.485 & 0.459 \\
\hline \pm S.D. & 0.0118 & 0.0214 & 0.0176 & 0.0100 & 0.0420 & 0.0507 \\
\hline
\end{tabular}

$\uparrow$ Significantly different to respective wild type skeletal muscle weight control $(\mathrm{p}<0.05)$ $\$$ Significantly different to respective wild type skeletal muscle weight control $(p<0.01)$ Significance was assessed by Student's t-test with Welch correction 


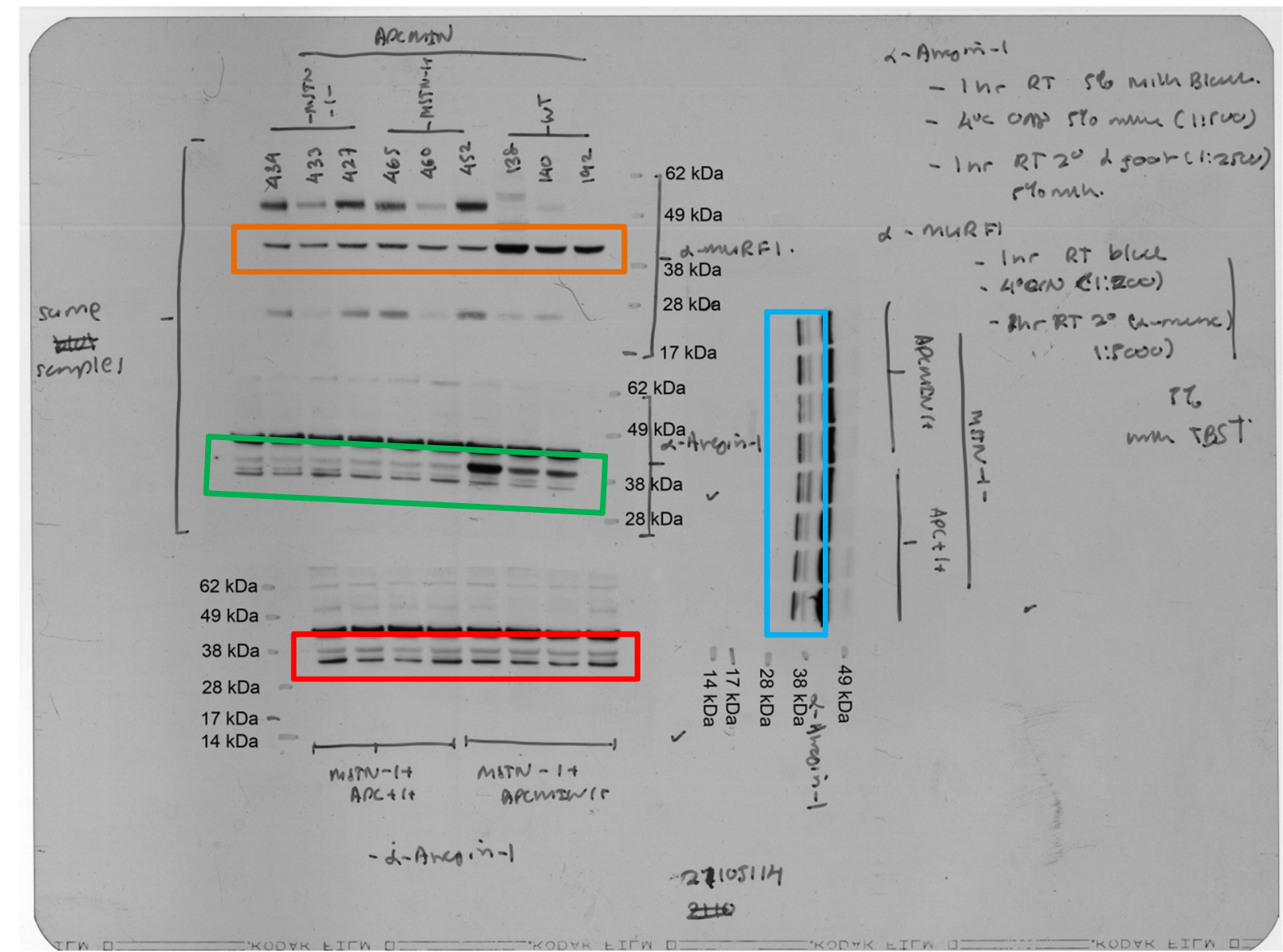

Figure 7.7.1 (Section 4.7, Figures 4.7.3A, 4.7.3B, and 4.7.3D): Immunoblots for Atrogin-1 and MuRF1.

A) The red box highlights the region used for Figure 4.7.3A (Atrogin-1).

B) The blue box highlights the region used for Figure 4.7.3B (Atrogin-1). Please note that the cropped image was rotated $90^{\circ} \mathrm{C}$ to the left, and reflected along the y-axis.

C) The green box highlights the region used for Figure 4.7.3D (Atrogin-1). The blot was slightly tilted to display the specific Atrogin-1 bands. This cropped image was reflected along the y-axis.

D) The orange box highlights the region used for Figure 4.7.3D (MuRF1). This cropped image was reflected along the y-axis.

Molecular weight markers are recorded on the image. Please note that there was a non-specific band above the specific $38 \mathrm{kDa}$ Atrogin-1 band. 


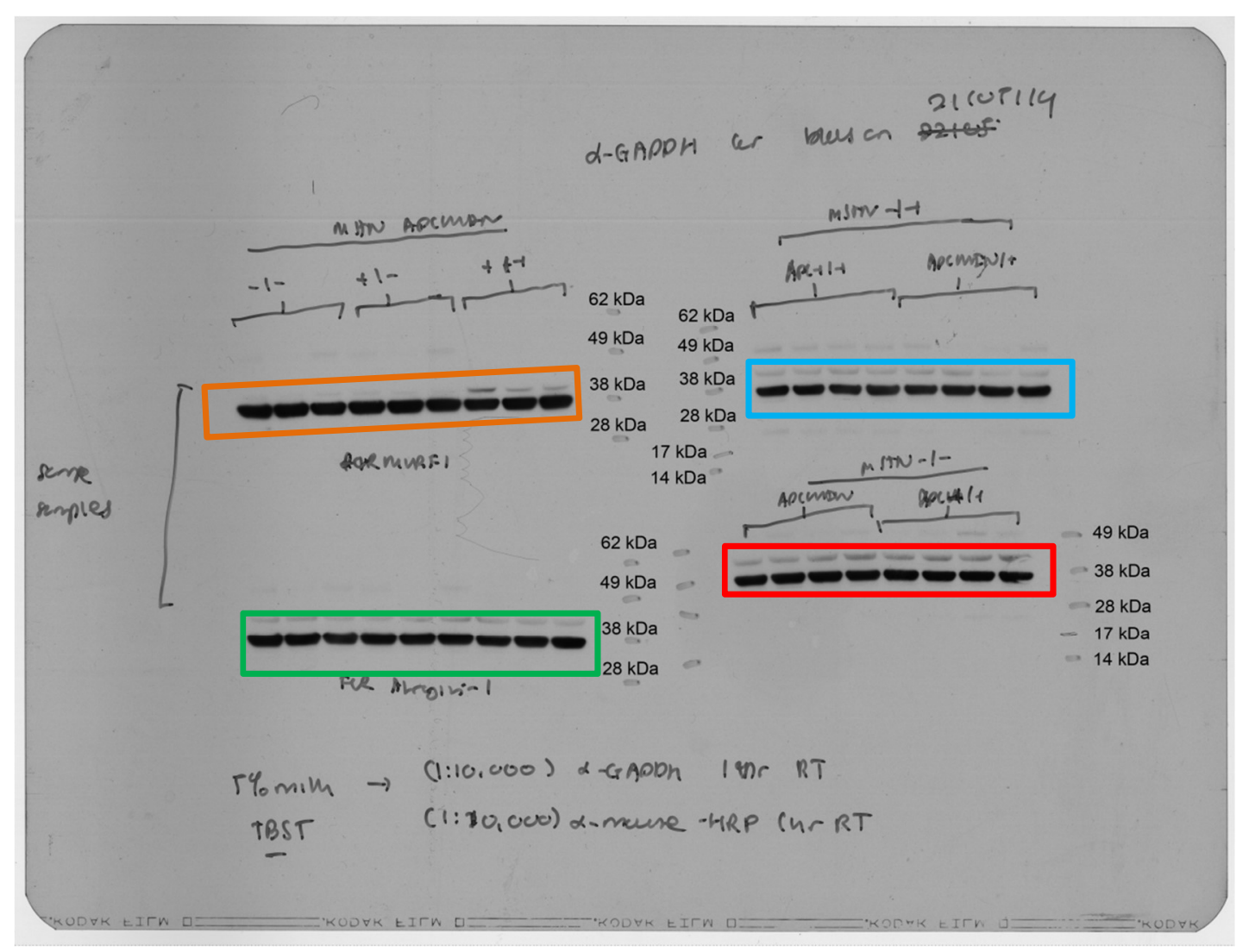

Figure 7.7.2 (Section 4.7, Figures 4.7.3A, 4.7.3B, and 4.7.3D): Immunoblots for GAPDH corresponding to the Atrogin-1 and MuRF1 immunoblots represented in Figure 7.7.1.

A) Blue box highlights the GAPDH immunoblot for the Atrogin-1 immunoblot in Figure 4.7.3A.

B) Red box highlights the GAPDH immunoblot for the Atrogin-1 immunoblot in Figure 4.7.3B.

C) Green box highlights the GAPDH immunoblot for the Atrogin-1 immunoblot in Figure 4.7.3D. This immunoblot is represented as the loading control in Figure 4.7.3D. The cropped imaged was reflected along the y-axis.

D) Orange box highlights the GAPDH immunoblot for the MuRF1 immunoblot in Figure 4.7.3D.

Molecular weight markers are recorded on the image. 


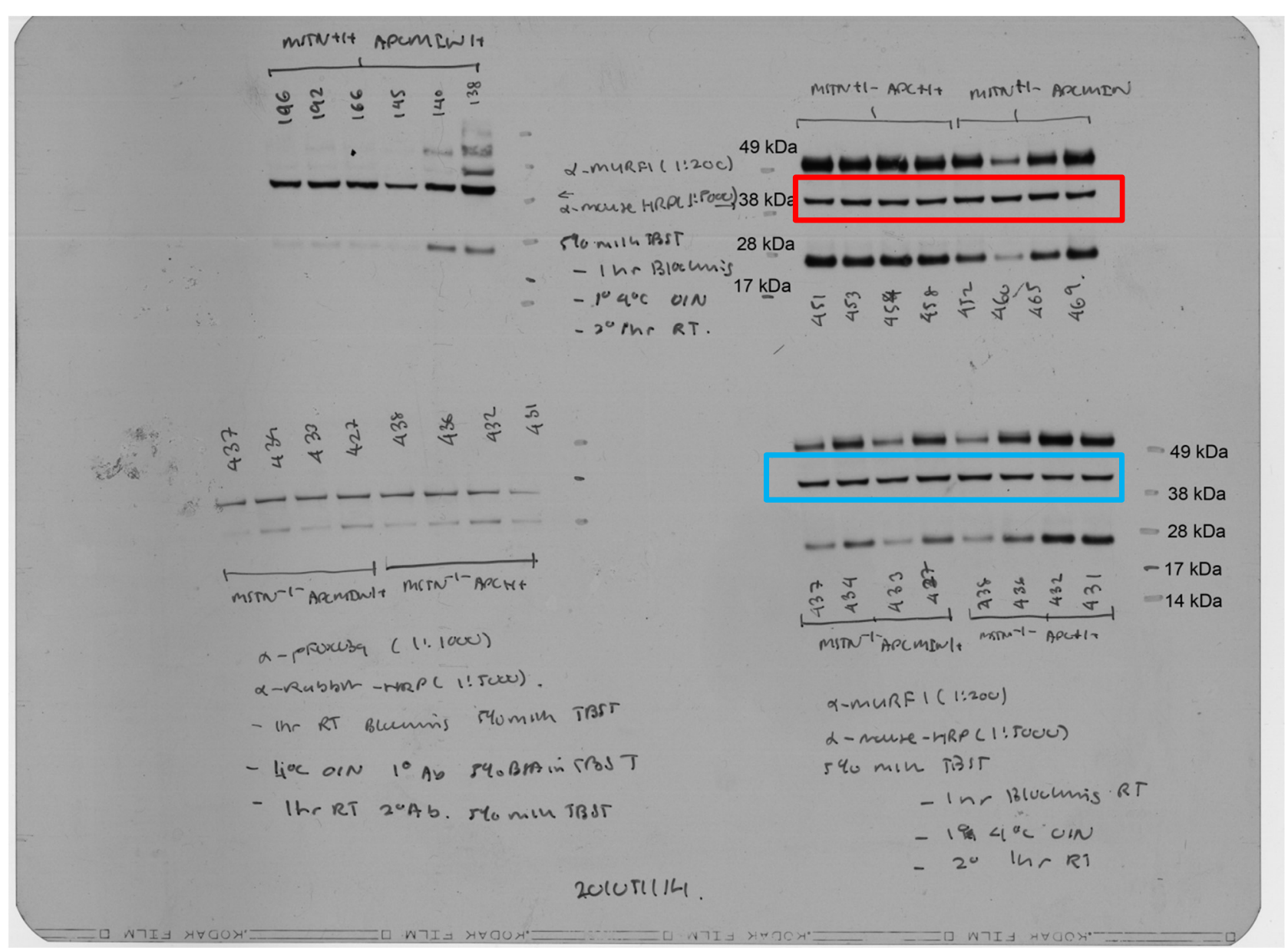

Figure 7.7.3 (Section 4.7, Figures 4.7.3A and 4.7.3B): Immunoblots for MuRF1.

A) Red box highlights the region used for Figure 4.7.3A (MuRF1).

B) Blue box highlights the region used for Figure 4.7.3B (MuRF1). Please note that the cropped image was reflected along the y-axis. Molecular weight markers are recorded on the image. 


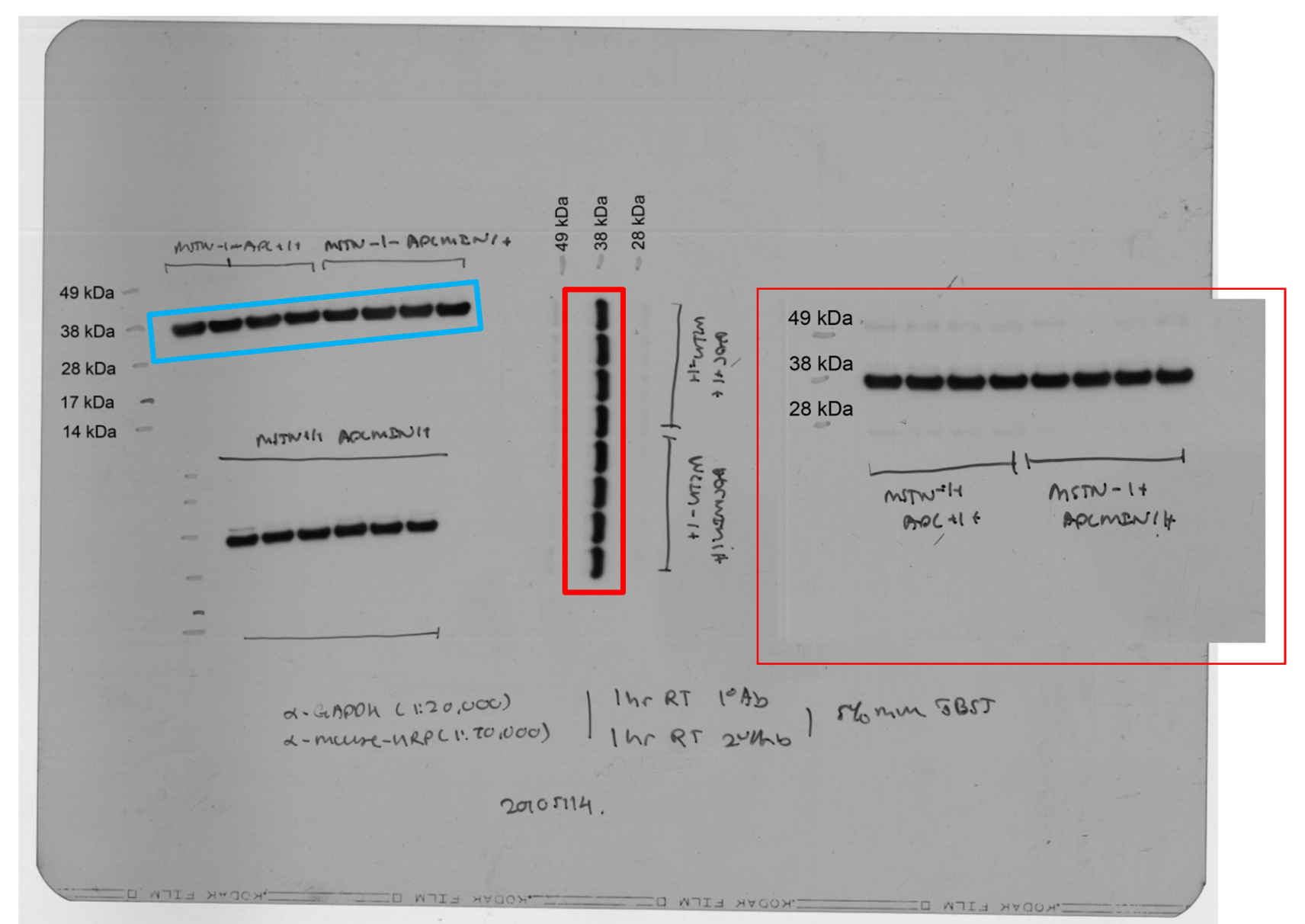

Figure 7.7.4 (Section 4.7, Figures 4.7.3A and 4.7.3B): Immunoblots for GAPDH corresponding to the MuRF1 immunoblots represented in Figure 7.7.3.

A) The red box highlights the GAPDH immunoblot for the MuRF1 immunoblot in Figure 4.7.3A. Cropped image was rotated $90^{\circ} \mathrm{C}$ to the right and reflected along the y-axis. This transformation is represented as an inset highlighted with a red border. This immunoblot is represented as the loading control in Figure 4.7.3A.

B) The blue box highlights the GAPDH immunoblot for the MuRF1 immunoblot in Figure 4.7.3B. The blot was slightly tilted to capture all the GAPDH bands. This immunoblot is represented as the loading control in Figure 4.7.3B.

Molecular weight markers are recorded on the image. 
Table 7.7.2 (Section 4.7, Figure 4.7.1): Tibia lengths for $\mathrm{Mstn}^{+/+}:: \mathrm{Apc}^{\mathrm{Min} /+}(\mathrm{n}=6), \mathrm{Mstn}^{+/-}$ $\because \mathrm{Apc}^{\mathrm{Min} /+}(\mathrm{n}=4)$, and $\mathrm{Mstn}^{-/-}: \mathrm{Apc}^{\mathrm{Min} /+}(\mathrm{n}=10)$ mice and their respective wild type controls, $M_{s t n}^{+/+}: \because \operatorname{Apc}^{+/+}(\mathrm{n}=6), M_{s t n}^{+/-}: \because \operatorname{Apc}^{+/+}(\mathrm{n}=6)$, and $M_{s t n}{ }^{-/}: \because A p c^{+/+}(\mathrm{n}=11)$ mice.

\begin{tabular}{|c|c|c|c|c|c|c|}
\hline & \multicolumn{2}{|c|}{$\mathrm{Mstn}^{+/+}$} & \multicolumn{2}{|c|}{$\mathrm{Mstn}^{+/-}$} & \multicolumn{2}{|c|}{$\mathrm{Mstn}^{-/-}$} \\
\hline & $A p c^{+/+}$ & $A p c^{M i n /+}$ & $A p c^{+/+}$ & $A p c^{\mathrm{Min} /+}$ & $A p c^{+/+}$ & $A p c^{\operatorname{Min} /+}$ \\
\hline $\begin{array}{l}\text { Tibia Length } \\
(\mathrm{mm})\end{array}$ & 17.84 & $17.25 \dagger$ & 17.64 & $17.47^{*}$ & $18.31 \bullet^{\circ}$ & $18.10 * . \bullet$ \\
\hline \pm S.D. & 0.31 & 0.10 & 0.43 & 0.04 & 0.27 & 0.23 \\
\hline
\end{tabular}

$\dagger$ Significantly different to respective wild type tibia length controls $(\mathrm{p}<0.01)$

Significance was assessed by Student's t-test with Welch correction

$\$$ Significantly different to $\mathrm{Mstn}^{+/+}:: A p c^{+/+}$tibia length $(\mathrm{p}<0.05)$

- Significantly different to $M s t n^{+/}: \because A p c^{+/+}$tibia length $(\mathrm{p}<0.01)$

* Significantly different to $M s n^{+/+}: \because A p c^{M i n /+}$ tibia length $(\mathrm{p}<0.001)$

- Significantly different to $M s{ }^{+1} \because: A p c^{M i n /+}$ tibia length control $(\mathrm{p}<0.001)$

Significance was assessed by one-way ANOVA followed by Tukey's post-hoc test 
7.8 The deletion of myostatin did not alter the intestinal tumor biology of the $\mathrm{Apc}^{\mathrm{Min} /+}$ mouse

A)

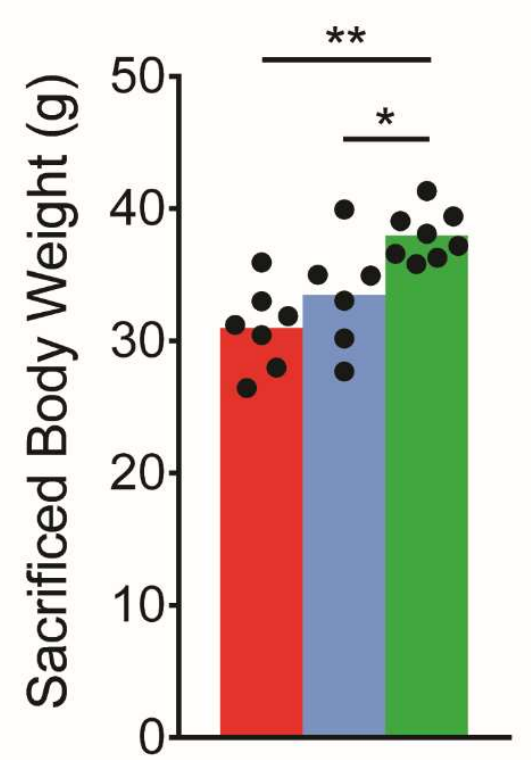

B)

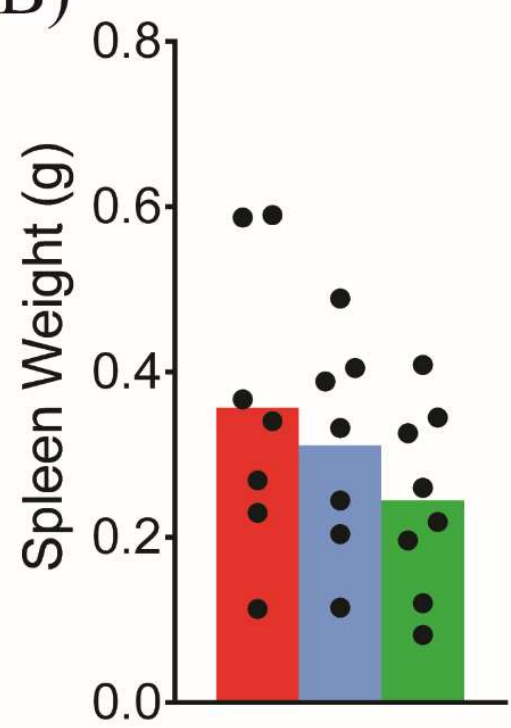

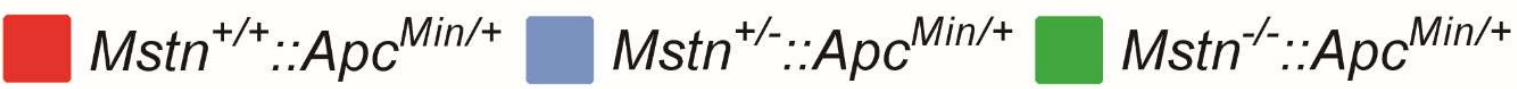

Figure 7.8.1 (Section 4.8, Figure 4.8.1): Sacrificed body weights and spleen weights from 20 to 21 week old $\mathrm{Mstn}^{+/+}:: \mathrm{Apc}^{\mathrm{Min} /+}, \mathrm{Mstn}^{+/-}:: \mathrm{Apc}^{\mathrm{Min} /+}$, and $\mathrm{Mstn}^{-/-}: \because \mathrm{Apc}^{\mathrm{Min} /+}$ mice.

A) Sacrificed body weights (grams) for $M_{s t n}^{+/+}: \because A p c^{M i n /+}(\mathrm{n}=7), M_{s t n}{ }^{+-} \because: A p c^{M i n /+}(\mathrm{n}=6)$, and $M_{s t n}-$ ${ }^{\prime} \because \because \operatorname{Apc}^{\mathrm{Min} /+}(\mathrm{n}=8)$ mice. Mice were between 20 to 21 weeks old. Significance was assessed by oneway ANOVA followed by Tukey's post-hoc test: ${ }^{*} p<0.05, * * p<0.01, * * * p<0.001$.

B) Spleen weights (grams) for $\operatorname{Mstn}^{+/+}: \because A p c^{\operatorname{Min} /+}(\mathrm{n}=7), \mathrm{Mstn}^{+/} \because: A p c^{\mathrm{Min} /+}(\mathrm{n}=7)$, and $\mathrm{Mstn}^{-/-}$ $\because \operatorname{Apc}^{\mathrm{Min} /+}(\mathrm{n}=8)$ mice. Mice were between 20 to 21 weeks old. Significance was assessed by oneway ANOVA followed by Tukey's post-hoc test: No significant difference was observed. 


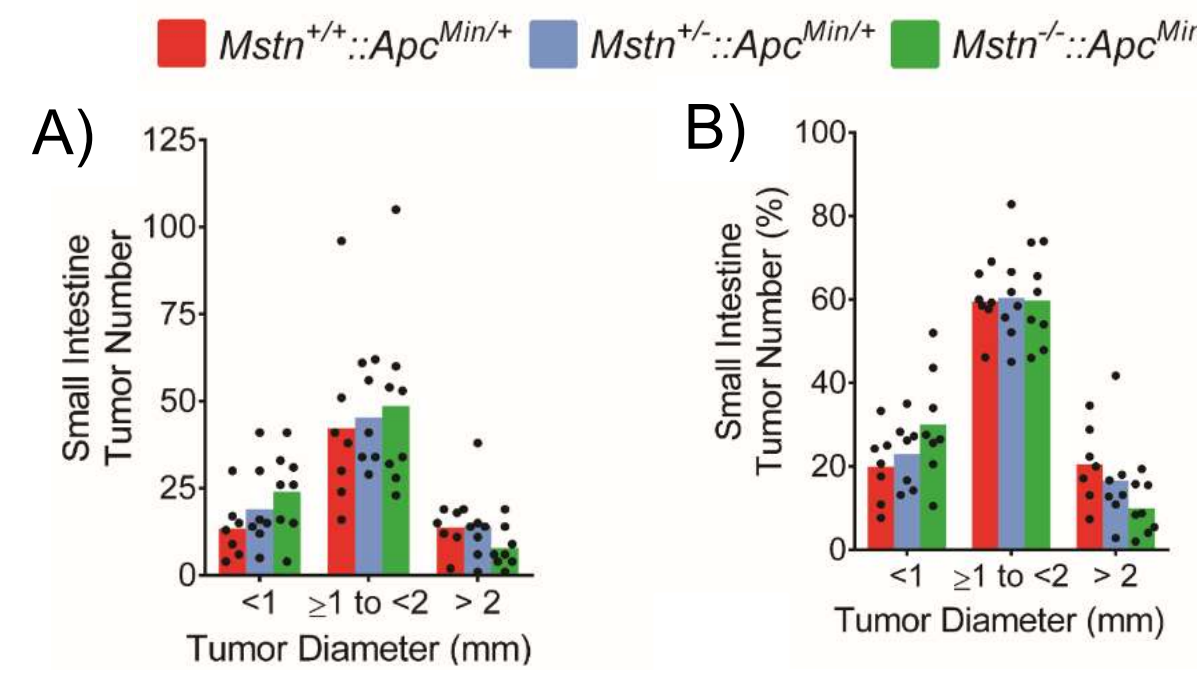

Figure 7.8.2 (Section 4.8, Figure 4.8.1B): Small intestine tumor diameter for $\mathrm{Mstn}^{+/+}:: \mathrm{Apc}^{\mathrm{Min} /+}, \mathrm{Mstn}^{+/-}:: \mathrm{Apc}^{\mathrm{Min} /+}$, and $\mathrm{Mstn}^{-/-}: \mathrm{Apc}^{\mathrm{Min} /+}$ mice.

A) Small intestine tumor diameter (mm) for $\operatorname{Mstn}^{+/+}: \because A p c^{M i n /+}(\mathrm{n}=7), \operatorname{Mstn}^{+/} \because: A p c^{M i n /+}(\mathrm{n}=7)$, and $M \operatorname{stn}^{-/}: \because A p c^{M i n /+}(\mathrm{n}=8)$ mice. Significance was assessed by one-way ANOVA followed by Tukey's post-hoc test: No significant difference was observed.

B) Small intestine tumor diameter (\%) for $\operatorname{Mstn}^{+/+}: \because A p c^{M i n /+}(\mathrm{n}=7), \operatorname{Mstn}^{+/-} \because: A p c^{M i n /+}(\mathrm{n}=7)$, and $\operatorname{Mstn}^{-/}:: A p c^{M i n /+}(\mathrm{n}=8)$ mice. Each data point represents the number of tumors that belong to the stated diameter over the total number of tumors per animal. Data was represented as a percentage. Significance was assessed by one-way ANOVA followed by Tukey's post-hoc test: No significant difference was observed. 
Table 7.8.1 (Section 4.8, Figure 4.8.1A and Figure 4.8.1B): Small intestine tumor number and diameter for $\mathrm{Mstn}^{+/+}:: \mathrm{Apc}^{\mathrm{Min} /+}, \mathrm{Mstn}^{+/-}:: \mathrm{Apc}^{\mathrm{Min} /+}$, and $\mathrm{Mstn}^{-/-}: \mathrm{Apc}^{\mathrm{Min} /+}$ mice.

\begin{tabular}{|c|c|c|c|}
\hline & $\operatorname{Mstn}^{+/+}:: A p c^{M i n /+}$ & $\operatorname{Mstn}^{+/}: \because A p c^{M i n /+}$ & $M_{s t n}{ }^{-/} \because: A p c^{M i n /+}$ \\
\hline Number of mice & 7 & 7 & 8 \\
\hline Number of tumors counted (n) & 486 & 549 & 644 \\
\hline Mean diameter $(\mathrm{mm})$ & 1.47 & 1.42 & 1.24 \\
\hline $\mathrm{SD} \pm$ & 0.6 & 0.7 & 0.5 \\
\hline Percentage Reduction & 0.00 & 2.92 & 15.33 \\
\hline Absolute Uncertainty $( \pm) \dagger$ & 0.0 & 1.9 & 9.4 \\
\hline
\end{tabular}

$\uparrow$ The uncertainty was calculated by calculating the fractional uncertainties then adding in quadrature. 


\subsection{The growth characteristics of $\mathrm{Mstn}^{-/}: \because \mathrm{Apc}^{\mathrm{Min} /+}$ cancer organoids}

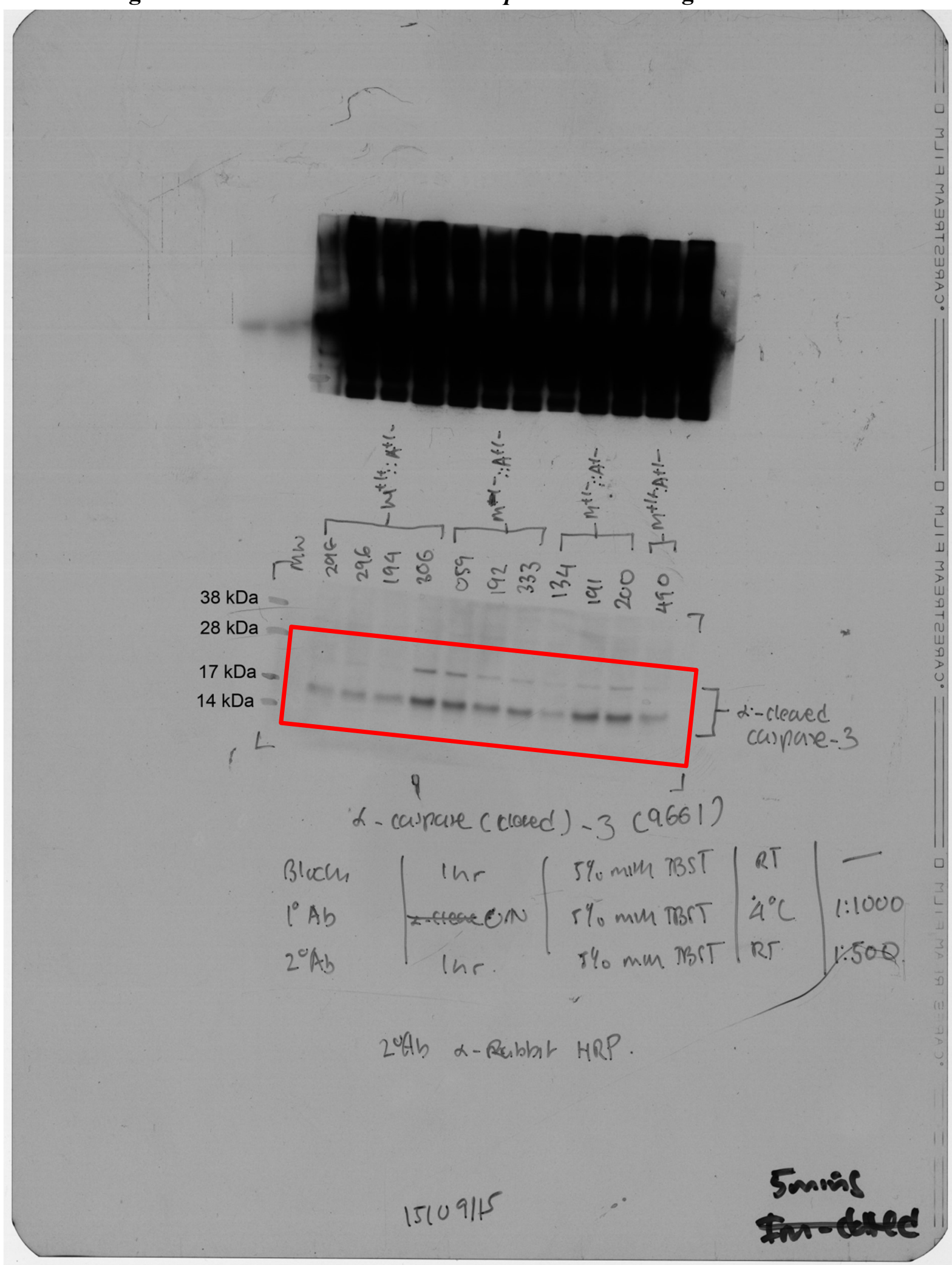

Figure 7.9.1 (Section 4.9, Figure 4.9.1B): Immunoblot for Cleaved Caspase-3. The red box highlights the region used for image preparation and densitometry calculation. Molecular weight markers are recorded on the image. 


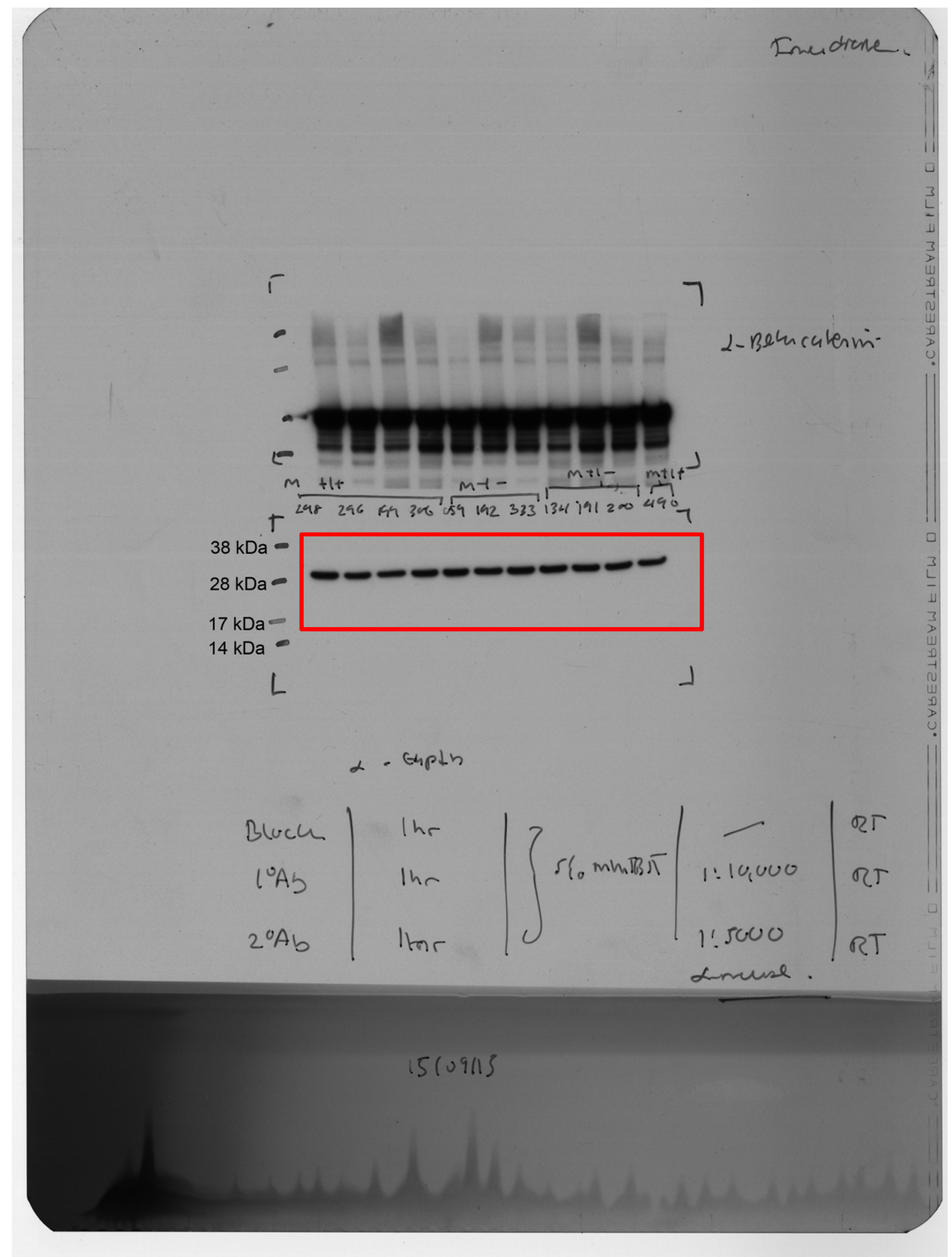

Figure 7.9.2 (Section 4.9, Figures 4.9.1B and 4.9.1C): Immunoblot for GAPDH for the Cleaved Caspase-3 immunoblot shown in Figure 7.9.1.

Red box highlights the region used for densitometry calculation and image preparation (Figure 4.9.1B). Molecular weight markers are recorded on the image. 
7.10 $\mathrm{Mstn}^{-/}$mice were resistant to colitis-induced colorectal carcinogenesis
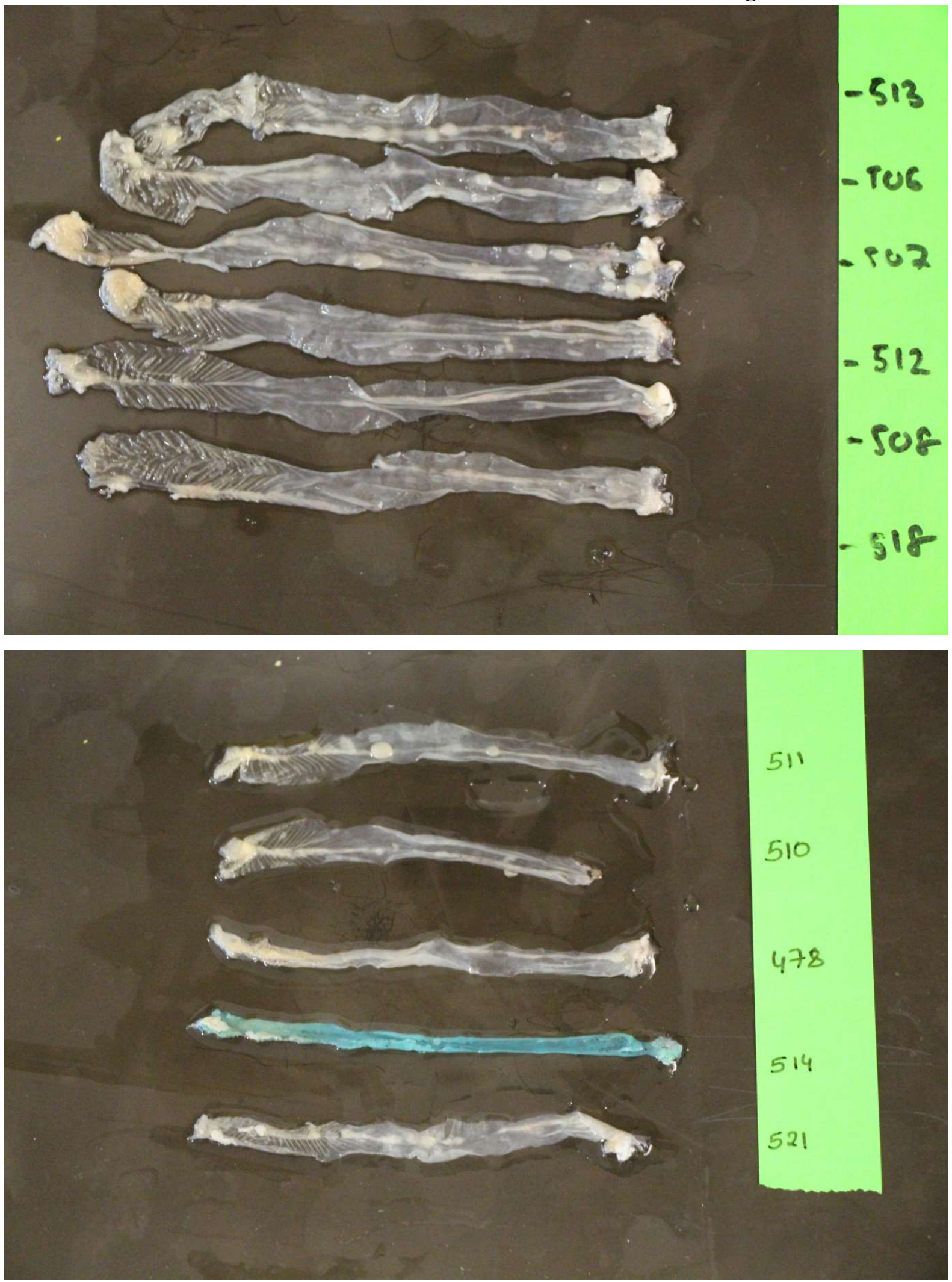

Figure 7.10.1 (Section 4.10, Figures 4.10.2A-C): Images of $10 \%$ NBF fixed $\mathrm{Mstn}^{+/+}$and $\mathrm{Mstn}^{-/}$colons used for macroscopic tumor analysis. 


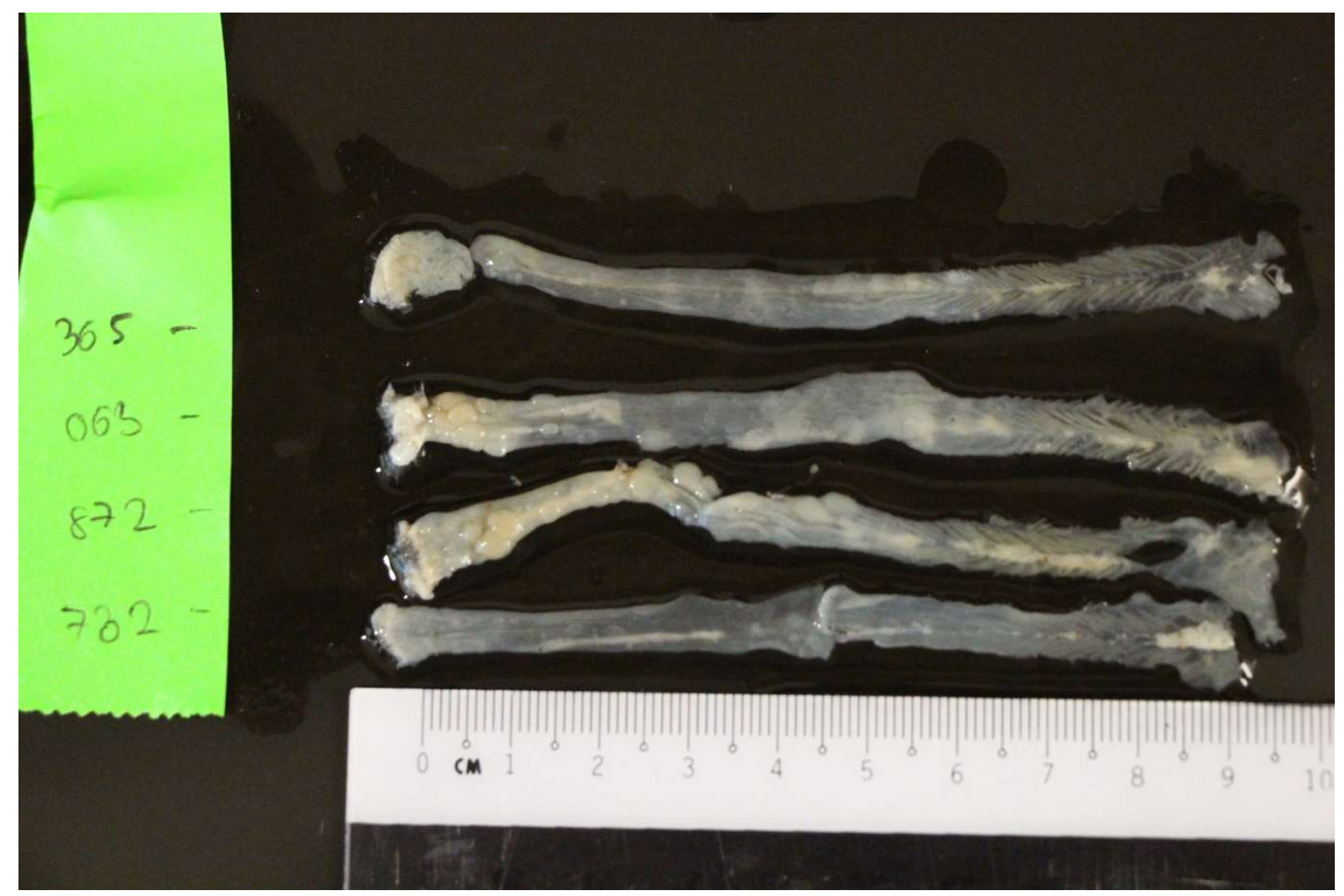

Figure 7.10.2 (Section 4.10, Figures 4.10.2A-C): Images of $10 \%$ NBF fixed $\mathrm{Mstn}^{+/+}$and $\mathrm{Mstn}^{-/}$colons used for macroscopic tumor analysis. 
Table 7.10.1 (Section 4.10, Figure 4.10.1E): Skeletal muscle weights for $\mathrm{Mstn}^{+/+}(\mathrm{n}=8)$ and $\mathrm{Mstn}^{-/}(\mathrm{n}=9)$ mice subjected to AOM/DSS model of colitis-induced colorectal carcinogenesis.

\begin{tabular}{lcc} 
& Mstn $+/+$ & Mstn- $/-$ \\
\hline TA weight $(\mathrm{g})$ & 0.0541 & $0.0947 \dagger$ \\
\pm S.D. & 0.0045 & 0.0074 \\
\hline EDL weight $(\mathrm{g})$ & 0.0102 & $0.0214 \dagger$ \\
\pm S.D. & 0.0019 & 0.0016 \\
\hline GAS weight $(\mathrm{g})$ & 0.1605 & $0.3077 \dagger$ \\
\pm S.D. & 0.0134 & 0.0230 \\
\hline SOL weight $(\mathrm{g})$ & 0.0075 & $0.0119 \dagger$ \\
\pm S.D. & 0.0013 & 0.0014 \\
\hline QUAD weight $(\mathrm{g})$ & 0.2517 & $0.4483 \dagger$ \\
\pm S.D. & 0.0179 & 0.0400 \\
\hline
\end{tabular}

$\uparrow$ Significantly different to $\mathrm{Mstn}^{+/+}$mice $(\mathrm{p}<0.001)$

Significance was assessed by Student's t-test with Welch correction 


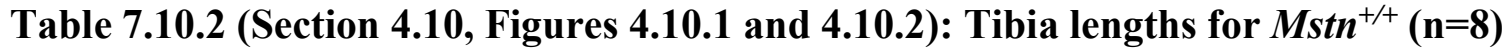
and $\mathrm{Mstn}^{-/}(\mathrm{n}=9)$ mice subjected to AOM/DSS model of colitis-induced colorectal carcinogenesis.

\begin{tabular}{lcc} 
& Mstn $+/+$ & Mstn-/- \\
\hline Tibia Length (mm) & 17.91 & 17.72 \\
\pm S.D. & 0.47 & 0.97 \\
\hline
\end{tabular}

Significance was assessed by Student's t-test with Welch correction. No significant difference was observed. 
7.11 The intestinal biology of the $\mathrm{Mstn}^{-/-}$mice
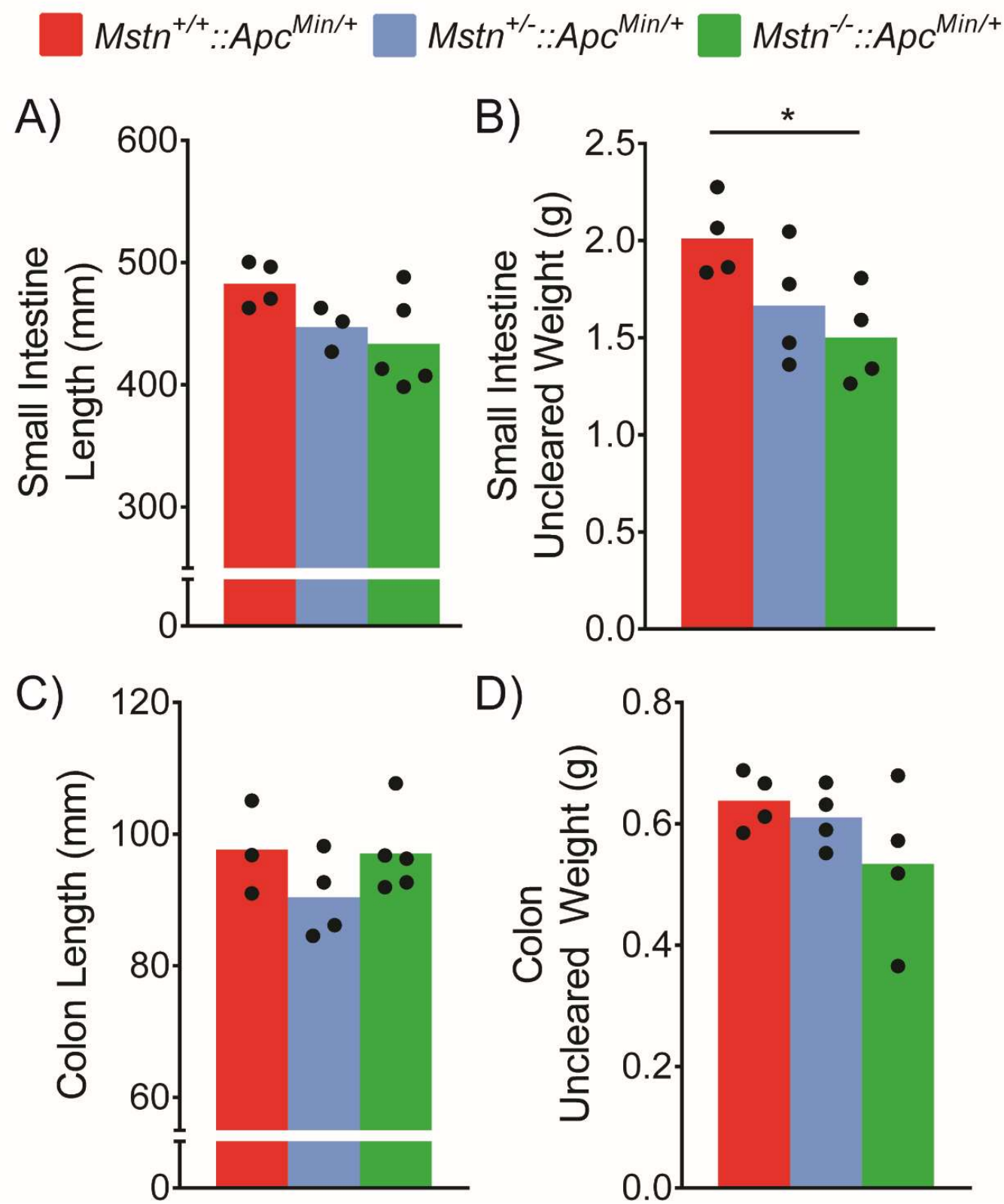

Figure 7.11.1 (Section 4.11, Figure 4.11.1): Intestinal biology of $\mathrm{Mstn}^{+/+}:: \mathrm{Apc}^{\mathrm{Min} /+}, \mathrm{Mstn}^{+/-}$ $\because A p c^{M i n /+}$, and $\mathrm{Mstn}^{-/-}:: A p c^{M i n /+}$ mice.

A) Small intestine length (mm) of $\mathrm{Mstn}^{+/+}: \because A p c^{\mathrm{Min} /+}(\mathrm{n}=4), \mathrm{Mstn}^{+/} \because: A p c^{\mathrm{Min} /+}(\mathrm{n}=3)$, and $\mathrm{Msth}^{-/-}$ $\because A p c^{M i n /+}(\mathrm{n}=5)$ mice. Mice were 19 to 20 weeks old. Significance was assessed by one-way ANOVA followed by Tukey's post-hoc test: No significant difference was observed.

B) Small intestine uncleared weight (grams) of $\operatorname{Mstn}^{+/+}: \because A p c^{M i n /+}(\mathrm{n}=4), M_{s t n}^{+/-}: \because A p c^{M i n /+}(\mathrm{n}=4)$, and $M_{s t n}{ }^{-/} \because: A p c^{M i n /+}(\mathrm{n}=4)$ mice. Mice were between 19 to 21 weeks of age. Significance was assessed by one-way ANOVA followed by Tukey's post-hoc test: ${ }^{*} p<0.05,{ }^{* *} p<0.01$, $* * * p<0.001$.

C) Colon length (mm) of $M_{s t n}^{+/+}: \because A p c^{M i n /+}(\mathrm{n}=3), M_{s t n}^{+/}: \because A p c^{M i n /+}(\mathrm{n}=4)$, and $M_{s t n}{ }^{-/}: \because A p c^{M i n /+}$ $(\mathrm{n}=5)$ mice. Mice were 19 to 20 weeks old. Significance was assessed by one-way ANOVA followed by Tukey's post-hoc test: No significant difference was observed.

D) Uncleared colon weight (grams) of $M_{s t n^{+/+}}: A p c^{M i n /+}(\mathrm{n}=4), M_{s t n}^{+/-}: \because A p c^{M i n /+}(\mathrm{n}=4)$, and Mstn ${ }^{-}$ ${ }^{\prime} \because A p c^{M i n /+}(\mathrm{n}=4)$ mice. Significance was assessed by one-way ANOVA followed by Tukey's posthoc test: No significant difference was observed. 


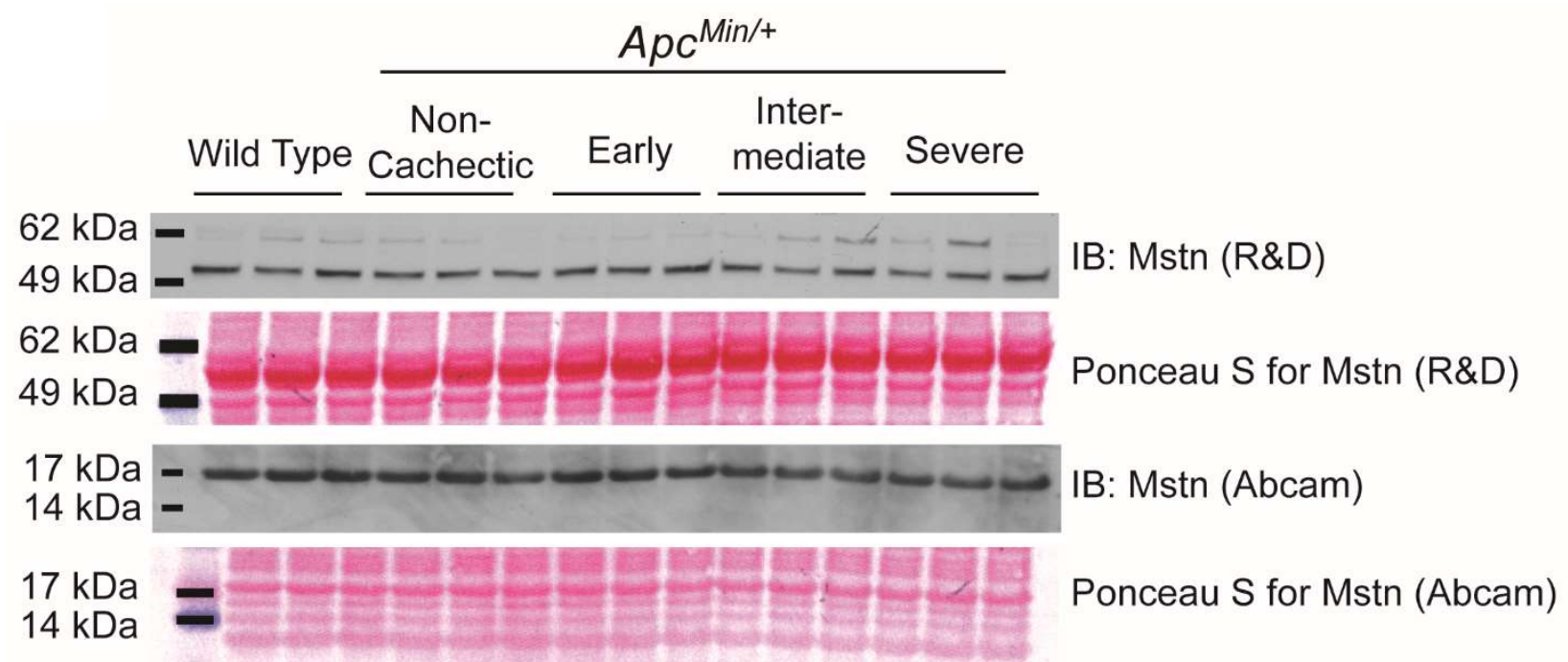

Figure 7.12.1 (Section 5.2): Myostatin protein expression levels in cachectic $\mathrm{Apc}^{\mathrm{Min} /+}$ mice were inconclusive.

Immunoblots for Myostatin (R\&D Systems and Abcam) for the GAS muscles of wild type, noncachectic, early, intermediate, and severely cachectic $A p c^{\mathrm{Min} /+}$ mice $(\mathrm{n}=3)$. Ponceau $\mathrm{S}$ was used as a loading control. The molecular weight makers are represented on the left side of the immunoblots. The immunoblots used for this figure and their corresponding Ponceau S loading controls are displayed in Figures 7.12.3 through 7.12.6. 


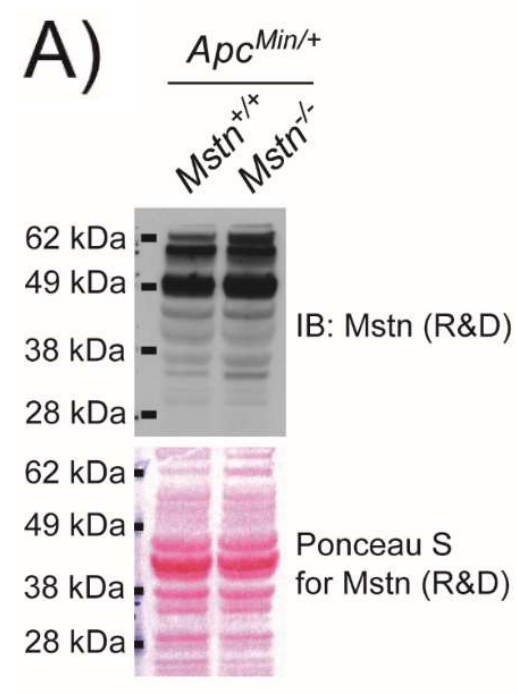

B)
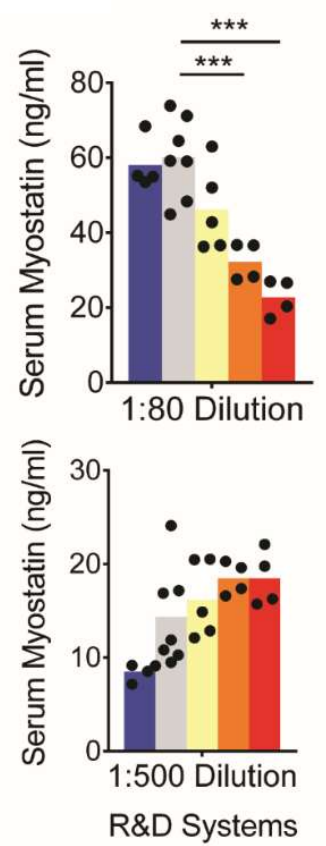

Wild Type

Non-cachectic

Early

Intermediate

Severe

。

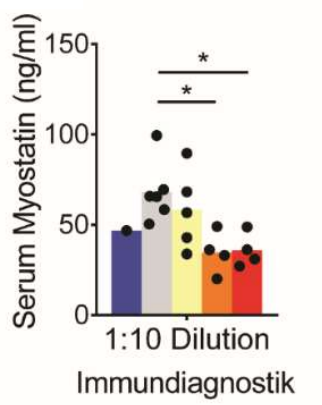

\section{Figure 7.12.2 (Section 5.2): Myostatin serum levels in cachectic Apc $^{\mathrm{Min} /+}$ mice were inconclusive.}

A) Immunoblot for Myostatin (R\&D Systems) for the GAS muscles of $\mathrm{Mstn}^{+/+}:: A p c^{\mathrm{Min} /+}$ (Mouse ID 140) and $\mathrm{Mstn}^{-1-}: \because A p c^{\mathrm{Min} /+}$ (Mouse ID 427) mice ( $\mathrm{n}=1$ ). Ponceau $\mathrm{S}$ was used as a loading control. The molecular weight makers are represented on the left side of the immunoblots. The immunoblots used for this figure and their corresponding Ponceau S loading controls are displayed in Figure 7.12.7 and 7.12.8.

B) Serum myostatin amounts $(\mathrm{ng} / \mu \mathrm{l})$ in wild type $(\mathrm{n}=4)$, non-cachectic $(\mathrm{n}=7)$, early $(\mathrm{n}=5)$, intermediate $(\mathrm{n}=4)$, and severely $(\mathrm{n}=4)$ cachectic $A p c^{\mathrm{Min} /+}$ mice. The R\&D Systems myostatin ELISA kit was used for this experiment. The top bar graph represents the results for serum diluted at 1:80. The bottom bar graph represents the results for serum diluted at 1:500. The final results represent myostatin concentration in the serum multiplied by the dilution factor. ELISA plate was read at $450 \mathrm{~nm}$ and corrected with a reference filter read at $550 \mathrm{~nm}$. The reference filter corrected absorbances were then subtracted by the blank absorbance. The background corrected absorbances were then interpolated onto the standard curve. The standard curve plotted with a linear absorbance and a linear concentration in Excel. Linear regression was used to create the standard curve. Significance was assessed by one-way ANOVA followed by Tukey's post-hoc test: $p<0.05$, $* * p<0.01, * * * p<0.001$. No significant difference was observed in for the myostatin ELISA performed with the serum diluted at 1:500.

C) Serum myostatin amounts $(\mathrm{ng} / \mu \mathrm{l})$ in wild type $(\mathrm{n}=4)$, non-cachectic $(\mathrm{n}=6)$, early $(\mathrm{n}=5)$, intermediate ( $\mathrm{n}=4)$, and severely $(\mathrm{n}=4)$ cachectic $A p c^{\mathrm{Min} / \mathrm{+}}$ mice. The Immundiagnostik myostatin ELISA kit was used for this experiment. The final results represent myostatin concentration in the serum multiplied by the dilution factor. ELISA plate was read at $450 \mathrm{~nm}$ and corrected with a reference filter read at $650 \mathrm{~nm}$. The reference filter corrected absorbances were then interpolated onto the standard curve. The standard curve plotted with a linear absorbance and logarithmic concentration in GraphPad Prism 6. Please note that the $0 \mathrm{ng} / \mu 1$ standard was set at 0.001 . A sigmoidal 4-parameter curve was used to create the standard curve. The reference corrected absorbances were interpolated to the standard curve using GraphPad Prism 6. Please note that 3 out the 4 wild type mice (Mouse ID 137, 912, and 998) demonstrated absorbances beyond the standard curve, and thus not included in the analysis. Significance was assessed by one-way ANOVA followed by Tukey's post-hoc test: $p<0.05, * * p<0.01, * * * p<0.001$. 


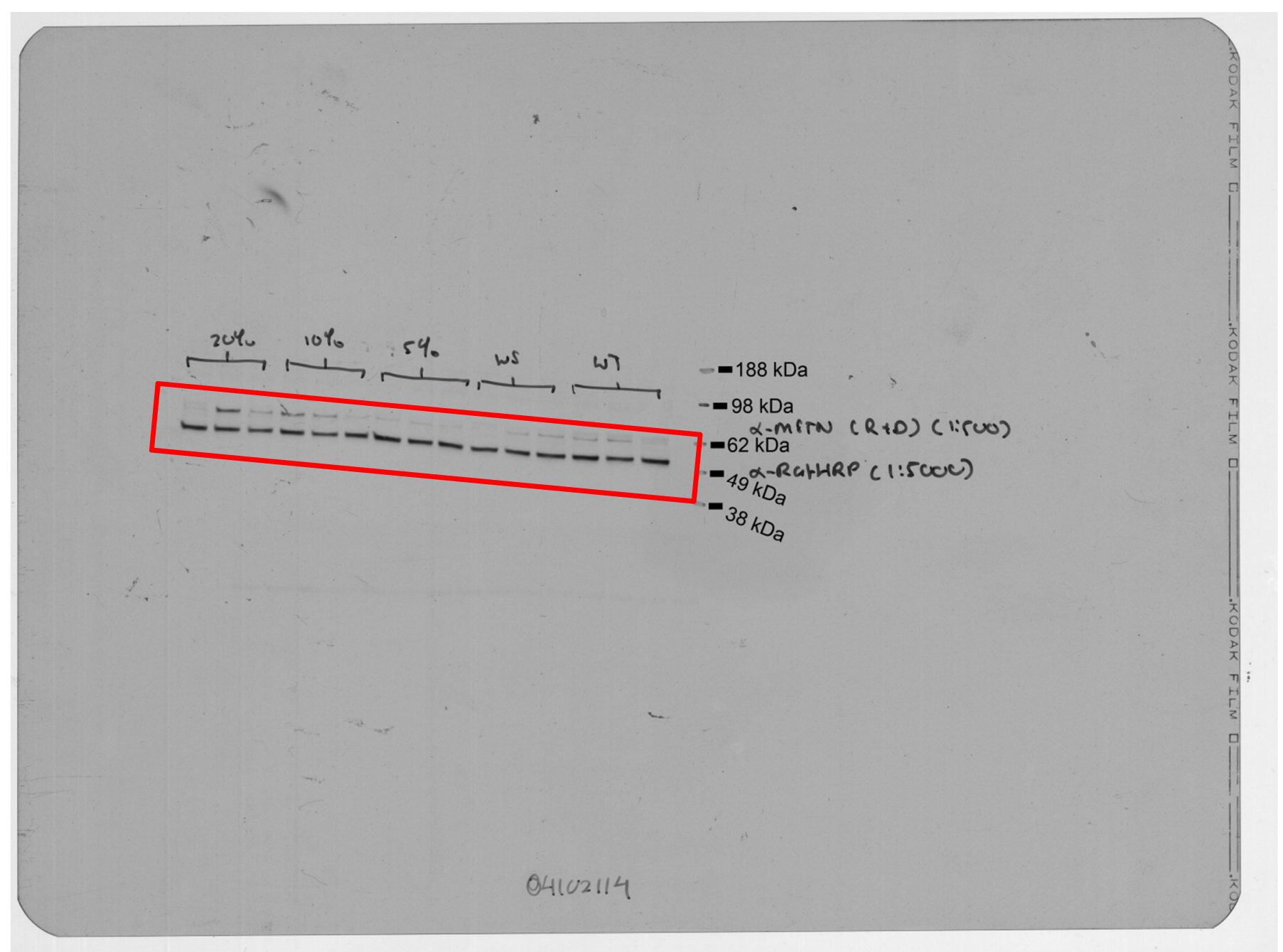

Figure 7.12.3 (Section 5.2, Figure 7.12.1): Immunoblot for Myostatin (R\&D Systems).

Red box highlights the region used for image preparation. Please note that the bottom half (below the $38 \mathrm{kDa}$ marker) was cut. The blot was slightly tilted to capture all the bands. Please note that the cropped image was reflected along the y-axis. Molecular weight markers are recorded on the image. 


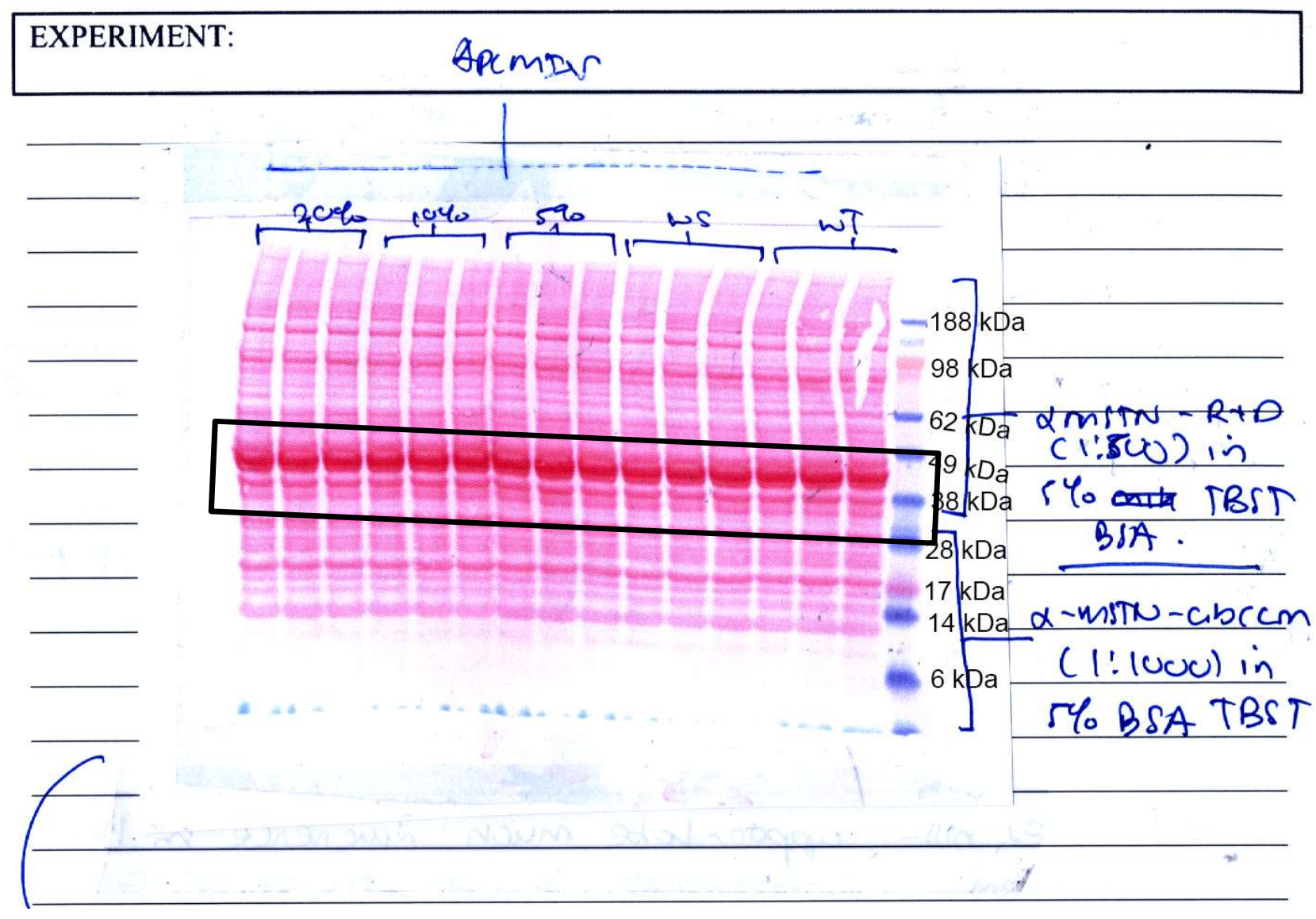

Figure 7.12.4 (Section 5.2, Figures 7.12.1 and 7.12.3): Ponceau $S$ for Myostatin (R\&D Systems) immunoblot in Figure 7.12.1 and Figure 7.12.3.

The black box highlights the region used for image preparation. The blot was slightly tilted to capture all the bands. Please note that the cropped image was reflected along the y-axis. Molecular weight markers are recorded on the image. 


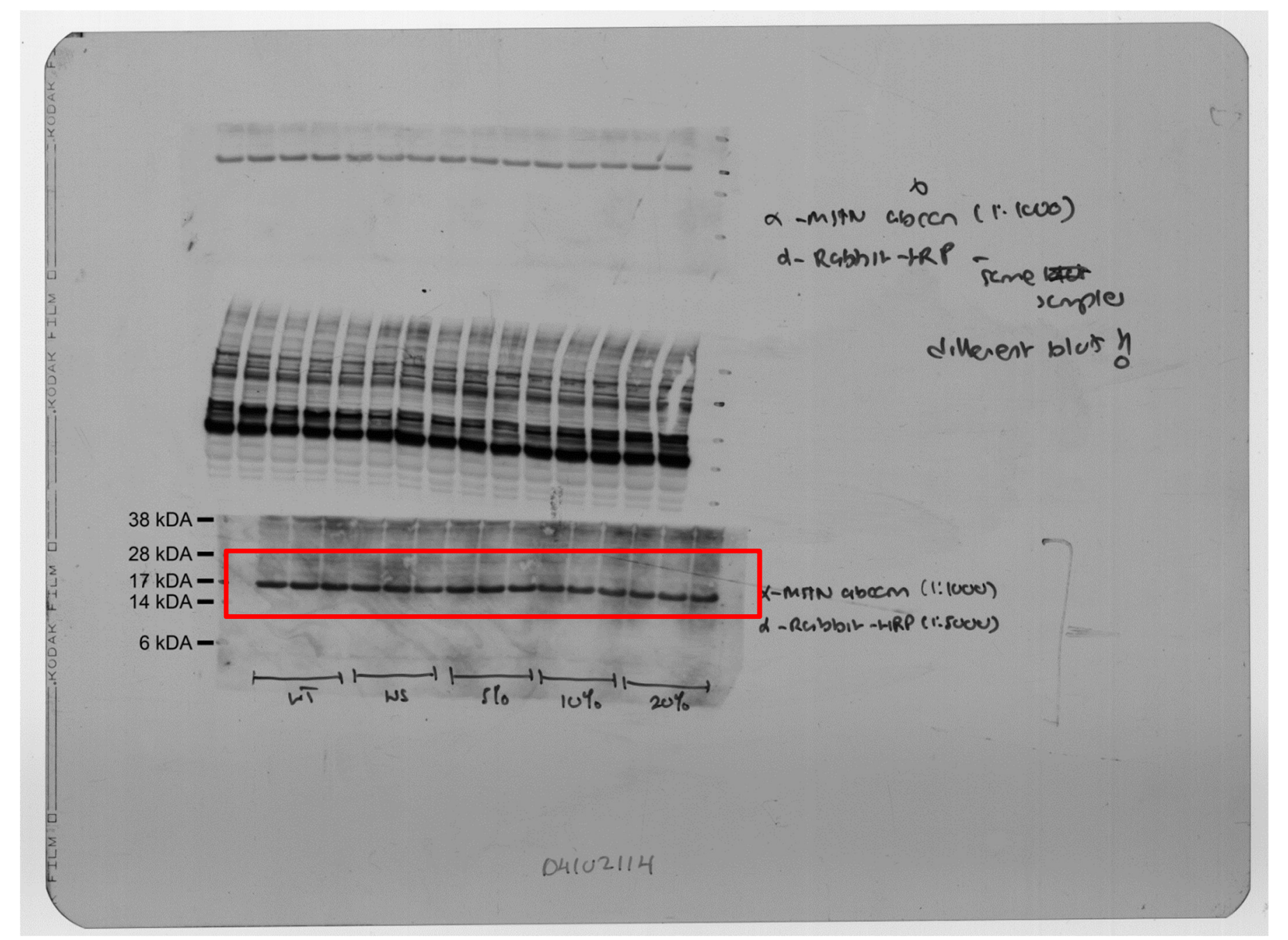

Figure 7.12.5 (Section 5.2, Figure 7.12.1): Immunoblot for Myostatin (Abcam).

Red box highlights the region used for image preparation. Please note that the top half of this immune (above the $38 \mathrm{kDa}$ molecular weight marker) was trimmed. Molecular weight markers are recorded on the image. 


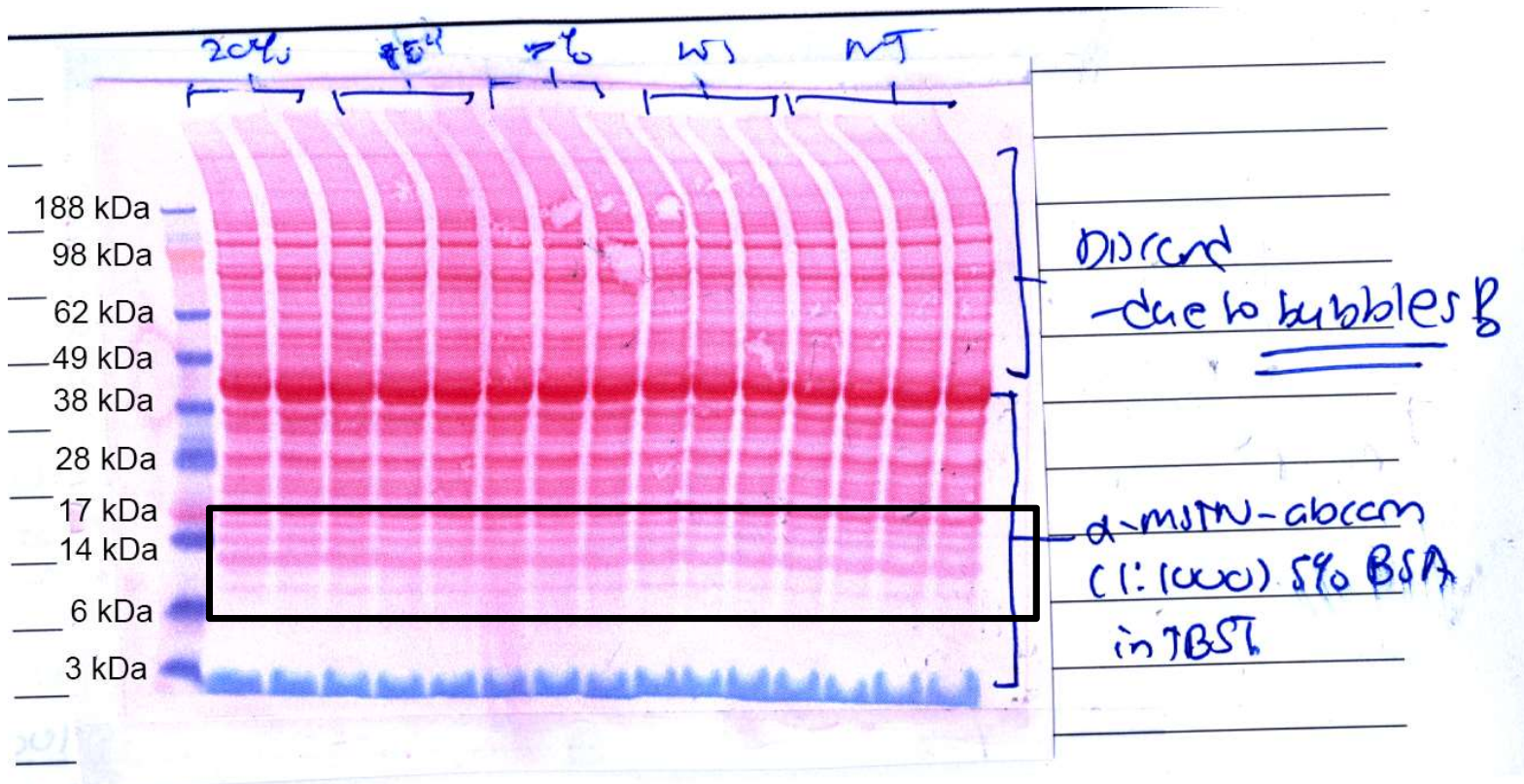

Figure 7.12.6 (Section 5.2, Figures 7.12.1 and 7.12.5): Ponceau $S$ for Myostatin (Abcam) immunoblot in Figure 7.12.1 and Figure 7.12.5.

The black box highlights the region used for image preparation. Molecular weight markers are recorded on the image. 


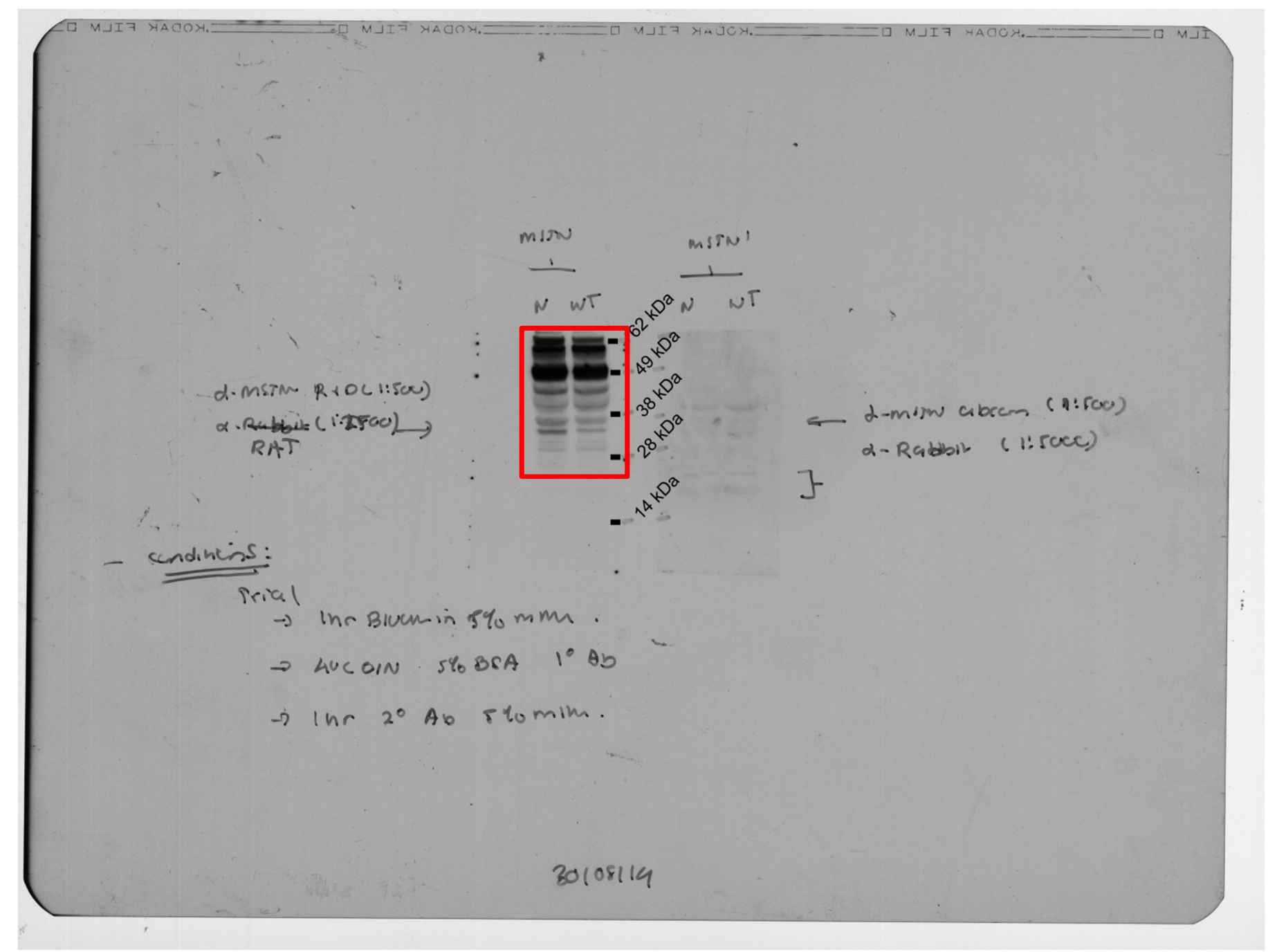

Figure 7.12.7 (Section 5.2, Figure 7.12.2A): Immunoblot for Myostatin (R\&D) (Figure 7.12.2A).

Red box highlights the region used for image preparation. Please note that the cropped image was reflected along the y-axis. Molecular weight markers are recorded on the image. 


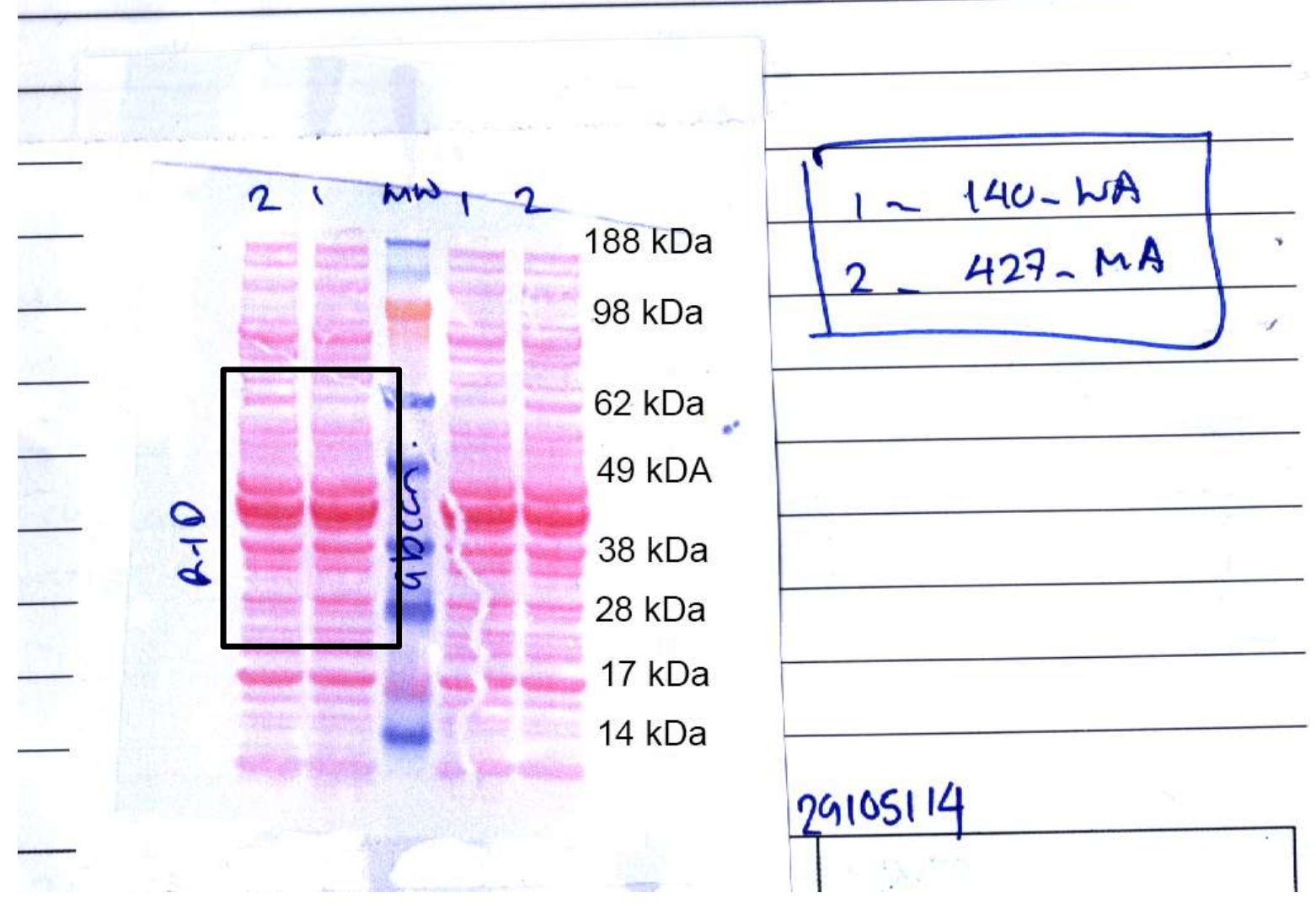

Figure 7.12.8 (Section 5.2, Figures 7.12.2A and 7.12.7): Ponceau $S$ for Myostatin (R\&D) immunoblot in Figure 7.12.2A and Figure 7.12.7.

The black box highlights the region used for image preparation. Please note that the cropped image was reflected along the y-axis. Molecular weight markers are recorded on the image. 


\subsection{Murine background and intestinal tumorigenesis}

$\mathrm{Mstn}^{+/+}:: \mathrm{Apc}^{\mathrm{Min} /+}$ (Colony Control) $\mathrm{Mstn}^{+/+}:: A p c^{\mathrm{Min} /+}(\mathrm{Het})$

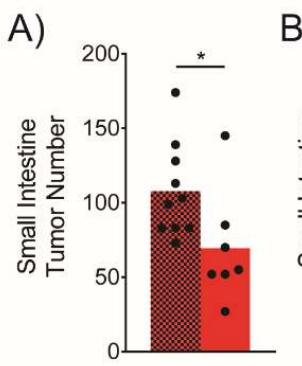

B)
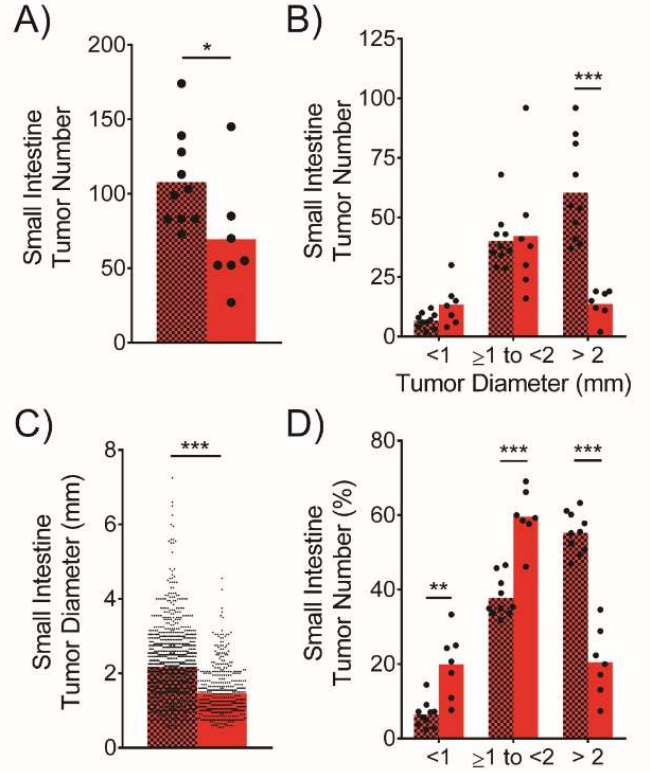

D)
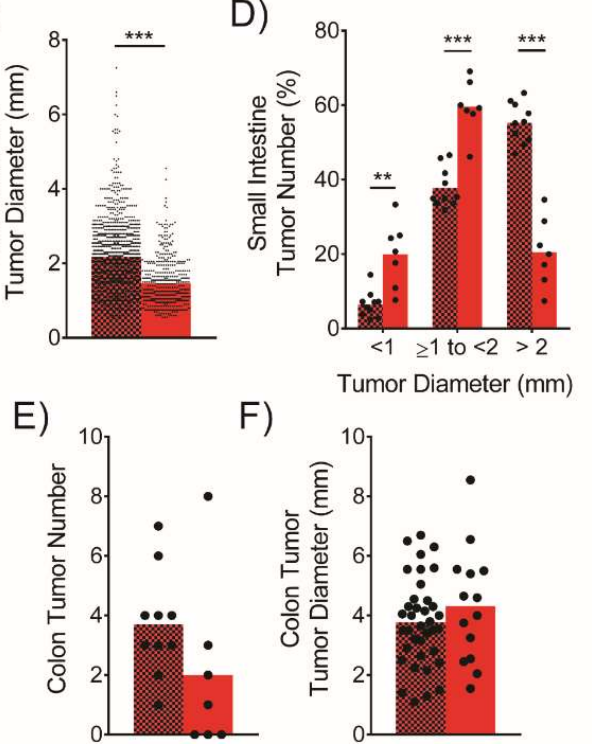

Figure 7.13.1 (Section 5.5): Colony control $\mathrm{Mstn}^{+/+}:: \mathrm{Apc}^{\mathrm{Min} /+}$ mice displayed an increase in small intestine tumor multiplicity and tumor size compared to $\mathrm{Mstn}^{+++}:: \mathrm{Apc}^{\mathrm{Min} /+}$ (Het) $^{\text {mice. }}$

A) Small intestine tumor number and B) Small intestine tumor diameter for colony control $M_{s t n^{+/+}}:: A p c^{M i n /+}(\mathrm{n}=10)$ and $M_{s t n}{ }^{+/+}:: A p c^{M i n /+}(\mathrm{Het})(\mathrm{n}=7)$ mice. Mice were 20 to 21 weeks old. Significance was assessed by Student's t-test with Welch correction: ${ }^{*} p<0.05, * * p<0.01, * * * p<0.001$. C) Average small intestine tumor diameter $(\mathrm{mm})$ for colony control $\mathrm{Mstn}^{+/+} \because: A p c^{\mathrm{Min} /+}(\mathrm{n}=10)$ and $M_{s t n}^{+/+}: \because A p c^{M i n /+}(H e t) \quad(n=7)$ mice. Mice were 20 to 21 weeks old. For the small intestine tumor diameter, $\mathrm{n}$ represents the number of mice, but each data point represents the diameter of one tumor. A data point represents the diameter of one tumor. Significance was assessed by Student's t-test with Welch correction: $* p<0.05, * * p<0.01, * * * p<0.001$.

D) Small intestine tumor diameter (\%) for colony control $\operatorname{Mstn}^{+/+}: \because A p c^{\operatorname{Min} /+} \quad(\mathrm{n}=10)$ and $\mathrm{Mstn}^{+/+}:: A p c^{\text {Min/+ }}{ }^{\text {Het })}(\mathrm{n}=7)$ mice. Mice were 20 to 21 weeks old. Each data point represents the number of tumors that belong to the stated diameter over the total number of tumors per animal. Data was represented as a percentage. Significance was assessed by Student's t-test with Welch correction: ${ }^{*} p<0.05, * * p<0.01, * * * p<0.001$.

E) Colon tumor number and F) Colon tumor diameter for colony control $\operatorname{Mstn}^{+/+}: \because A p c^{M i n /+}(\mathrm{n}=10)$ and $\mathrm{Mstn}^{+/+}: \because A p c^{\text {Min/+ }(\mathrm{Het})}(\mathrm{n}=7)$ mice. For the colon tumor diameter, $\mathrm{n}$ represents the number of mice, but each data point represents the diameter of one tumor. Mice were 20 to 21 weeks old. Significance was assessed by Student's t-test with Welch correction: No significant difference was observed. 

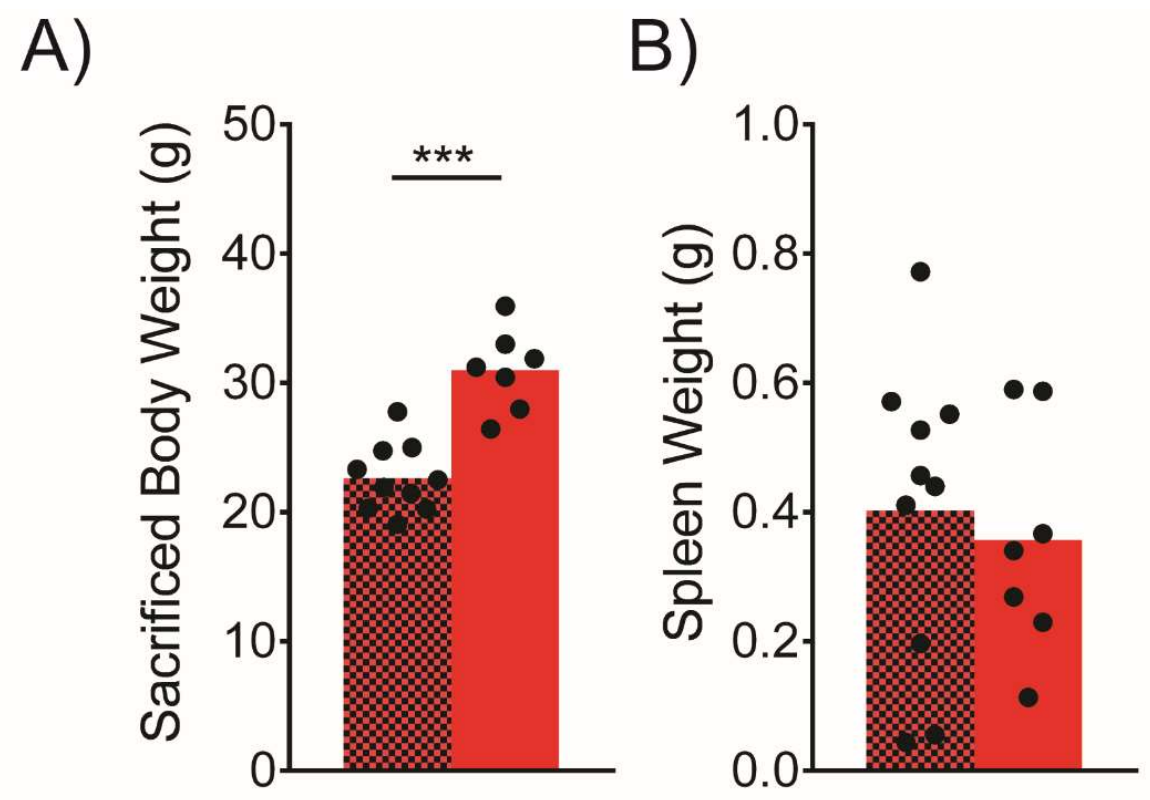

$\mathrm{Mstn}^{+/+}:: A p c^{\mathrm{Min} /+}$ (Colony Control) $\quad \mathrm{Mstn}^{+/+}: . A p c^{\mathrm{Min} /+}(\mathrm{Het})$

Figure 7.13.2 (Section 5.5, Figure 7.13.1): Sacrificed body weights and spleen weights for colony control $\mathrm{Mstn}^{+/+}:: \mathrm{Apc}^{\mathrm{Min} /+}$ and $\mathrm{Mstn}^{+/+}:: \mathrm{Apc}^{\mathrm{Min} /+(\mathrm{Het})}$ mice.

A) Sacrificed body weights (grams) for colony control $\mathrm{Mstn}^{+/+}: \because A p c^{\mathrm{Min} /+}(\mathrm{n}=10)$ and $\mathrm{Mstn}^{+/+}: \because A p c^{\mathrm{Min} /+}$ ${ }^{(H e t)}(\mathrm{n}=7)$ mice used in Figure 7.13.1. Mice were 20 to 21 weeks old. Significance was assessed by Student's t-test with Welch correction: ${ }^{*} p<0.05,{ }^{*} p<0.01, * * * p<0.001$.

B) Spleen weights (grams) for colony control Mstn ${ }^{++}: \because A p c^{M i n /+}(\mathrm{n}=10)$ and $\mathrm{Mstn}^{+/+}:: A p c^{\mathrm{Min} /+}$ (Het) $(\mathrm{n}=7)$ mice used in Figure 7.13.1. Mice were 20 to 21 weeks old. Significance was assessed by Student's t-test with Welch correction: No significant difference was observed. 


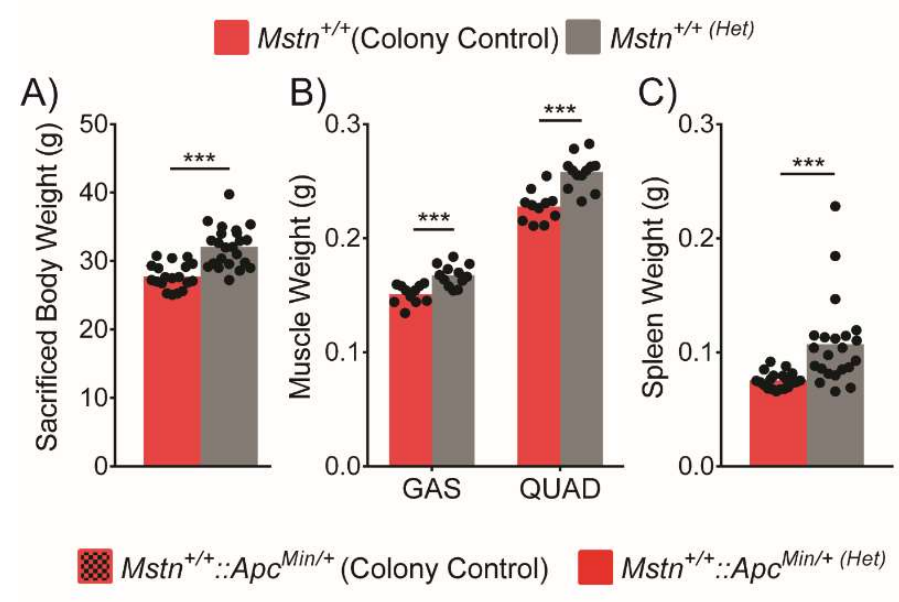

D)

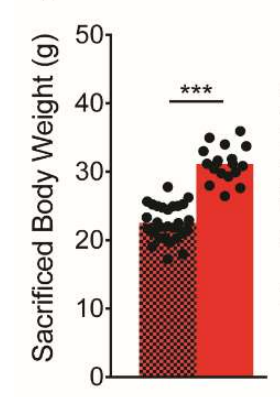

E)

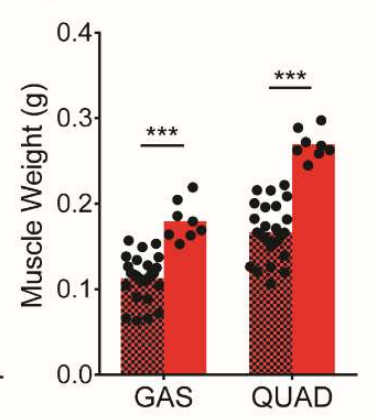

F)

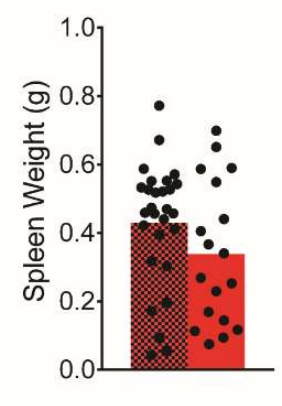

Figure 7.13.3 (Section 5.5): Body weights, skeletal muscle weights, and spleen weights for colony control $\mathrm{Mstn}^{+/+}:: \mathrm{Apc}^{+/+}$and $\mathrm{Mstn}^{+/+}:: \mathrm{Apc}^{+/+}(\mathrm{Het})$ mice, and $\mathrm{Mstn}^{+/+}:: A p c^{+/+(H e t)}$ and $\mathrm{Mstn}^{+/+}:: \mathrm{Apc}^{\mathrm{Min} /+}(\mathrm{Het})$ mice.

A) Sacrificed body weights (grams) for colony control $M s t n^{+/+}: \because A p c^{+/+}$mice $(\mathrm{n}=19)$ and $M_{s t n}^{+/+}: \because A p c^{+/+(H e t)}(\mathrm{n}=23)$. Mice were between 18 to 22 weeks of age. Significance was assessed by Student's t-test with Welch correction: ${ }^{*} p<0.05,{ }^{*} p<0.01, * * * p<0.001$.

B) Gastrocnemius (GAS) and quadriceps (QUAD) weights (grams) for colony control $\mathrm{Mstn}^{+/+}:: \mathrm{Apc}^{+/+}$ mice $(\mathrm{n}=11)$ and $\mathrm{Mstn}^{++}: \because A p c^{+++(\mathrm{Het})}(\mathrm{n}=12)$. Mice were between 18 to 20 weeks of age. Significance was assessed by Student's t-test with Welch correction: * $p<0.05$, ${ }^{*} p<0.01,{ }^{*} * *<0.001$.

C) Spleen weights (grams) for colony control $\mathrm{Mstn}^{+/+}: \because \mathrm{Apc}^{+/+}$mice $(\mathrm{n}=20)$ and $\mathrm{Mstn}^{+/+}: \because \mathrm{Apc}^{+/+}$(Het) $(\mathrm{n}=22)$. Mice were between 18 to 22 weeks of age. Significance was assessed by Student's t-test with Welch correction: $* p<0.05, * * p<0.01, * * * p<0.001$.

D) Sacrificed body weights (grams) for colony control $\operatorname{Mstn}^{+/+}: \because A p c^{M i n /+}$ mice $(\mathrm{n}=28)$ and

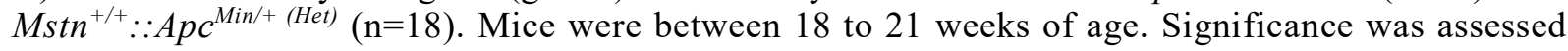
by Student's t-test with Welch correction: ${ }^{*} p<0.05, * * p<0.01, * * * p<0.001$.

E) Gastrocnemius (GAS) and quadriceps (QUAD) weights (grams) for colony control $\operatorname{Mstn}^{+/+}: \because A p c^{\text {Min/+ }}(\mathrm{n}=26)$ and Mstn $^{+++}: \because A p c^{\text {Min/+ }(H e t)}(\mathrm{n}=8)$. Mice were between 18 to 20 weeks of age. Significance was assessed by Student's t-test with Welch correction: ${ }^{*} p<0.05, * * p<0.01,{ }^{*} * * p<0.001$. F) Spleen weights (grams) for colony control $\mathrm{Mstn}^{+/+}: \because A p c^{\mathrm{Min} /+}(\mathrm{n}=20)$ and $\mathrm{Mstn}^{+/+}:: A p c^{\mathrm{Min} /+}(\mathrm{Het})$ $(\mathrm{n}=18)$. Mice were between 18 to 21 weeks of age. Significance was assessed by Student's t-test with Welch correction: No significant difference was observed. 
Table 7.13.1 (Section 5.5, Figure 7.13.3E): Skeletal muscle weights for colony control $\mathrm{Mstn}^{+/+}:: \mathrm{Apc}^{\mathrm{Min} /+}(\mathrm{n}=26)$ and $\mathrm{Mstn}^{+/+}:: \mathrm{Apc}^{\mathrm{Min} /+}(\mathrm{Het})(\mathrm{n}=8)$ mice.

\begin{tabular}{lcc} 
& \multicolumn{2}{c}{ Mstr $^{+/+}:: A^{M} c^{\text {Min } /+}$} \\
\cline { 2 - 3 } & Colony Control & Heterozygote Breeding \\
& & Scheme \\
\hline TA weight $(\mathrm{g})$ & 0.0358 & $0.0519 \dagger$ \\
\pm S.D. & 0.0068 & 0.0053 \\
\hline EDL weight $(\mathrm{g})$ & 0.0075 & 0.0107 \\
\pm S.D. & 0.0022 & 0.0038 \\
\hline GAS weight $(\mathrm{g})$ & 0.1131 & $0.1798 \dagger$ \\
\pm S.D. & 0.0261 & 0.0226 \\
\hline SOL weight $(\mathrm{g})$ & 0.0070 & 0.0067 \\
\pm S.D. & 0.0011 & 0.0009 \\
\hline QUAD weight $(\mathrm{g})$ & 0.1669 & $0.2694 \dagger$ \\
\pm S.D. & 0.0321 & 0.0169 \\
\hline
\end{tabular}

$\uparrow$ Significantly different to colony control $M s{ }^{+/+}: \because A p c^{M i n /+}$ mice $(\mathrm{p}<0.001)$

Significance was assessed by Student's t-test with Welch correction

Mice were between 18 to 20 weeks old 
Table 7.13.2 (Section 5.5, Figures 7.13.3D-F): Tibia length $(\mathrm{mm})$ for colony control $\mathrm{Mstn}^{+/+}:: \mathrm{Apc}^{\mathrm{Min} /+}(\mathrm{n}=10)$ and $\mathrm{Mstn}^{+/+}:: \mathrm{Apc}^{\mathrm{Min} /+}(\mathrm{n}=9)$ mice.

\begin{tabular}{lcc} 
& \multicolumn{2}{c}{$\operatorname{Mstn}^{+/+}:: A p c^{M i n /+}$} \\
\cline { 2 - 3 } & Colony Control & $\begin{array}{c}\text { Heterozygote } \\
\text { Breeding Scheme }\end{array}$ \\
\hline Tibia Length $(\mathrm{mm})$ & 17.52 & 18.07 \\
\pm S.D. & 0.41 & 0.23 \\
\hline
\end{tabular}

Significance was assessed by Student's t-test with Welch correction. No significant difference was observed. Mice were between 18 to 20 weeks old 
Table 7.13.3 (Section 5.5, Figure 7.13.3B): Skeletal muscle weights for colony control $\mathrm{Mstn}^{+/+}:: \mathrm{Apc}^{+/+}$mice $(\mathrm{n}=11)$ and $\mathrm{Mstn}^{+/+}:: \mathrm{Apc}^{+/+}(\mathrm{Het})(\mathrm{n}=12)$ mice.

$$
\mathrm{Mstn}^{+/+}
$$

Colony Control Heterozygote Breeding Scheme

\begin{tabular}{lcc}
\hline TA weight $(\mathrm{g})$ & 0.0480 & 0.0511 \\
\pm S.D. & 0.0026 & 0.0051 \\
\hline EDL weight $(\mathrm{g})$ & 0.0098 & 0.0089 \\
\pm S.D. & 0.0014 & 0.0022 \\
\hline GAS weight $(\mathrm{g})$ & 0.1511 & $0.1675 \dagger$ \\
\pm S.D. & 0.0083 & 0.0094 \\
\hline SOL weight $(\mathrm{g})$ & 0.0078 & 0.0072 \\
\pm S.D. & 0.0011 & 0.0015 \\
\hline QUAD weight $(\mathrm{g})$ & 0.2278 & $0.2579 \dagger$ \\
\pm S.D. & 0.0134 & 0.0147 \\
\hline
\end{tabular}

$\dagger$ Significantly different to colony control $\mathrm{Mstn}^{+/+}$mice $(\mathrm{p}<0.001)$ Significance was assessed by Student's t-test with Welch correction Mice were between 18 to 20 weeks old 
Table 7.13.4 (Section 5.5, Figure 7.13.3A-C): Tibia length $(\mathrm{mm})$ for colony control $\mathrm{Mstn}^{+/+}:: \mathrm{Apc}^{+/+}(\mathrm{n}=10)$ and $\mathrm{Mstn}^{+/+}:: \mathrm{Apc}^{+/+}(\mathrm{Het})(\mathrm{n}=14)$ mice.

\begin{tabular}{lcc} 
& \multicolumn{2}{c}{$M^{2} \operatorname{stn}^{+/+}:: A p c^{+/+}$} \\
\cline { 2 - 3 } & Colony Control & $\begin{array}{c}\text { Heterozygote } \\
\text { Breeding Scheme }\end{array}$ \\
\hline Tibia Length $(\mathrm{mm})$ & 17.67 & $18.28 \dagger$ \\
\pm S.D. & 0.33 & 0.37 \\
\hline
\end{tabular}

$\dagger$ Significantly different to colony control $\mathrm{Mstn}^{+/+}:: A p c^{\operatorname{Min} /+}$ mice $(\mathrm{p}<0.001)$ Significance was assessed by Student's t-test with Welch correction.

Mice were between 18 to 20 weeks old 


\section{$\mathrm{Mstn}^{+/+}\left(\right.$Colony Control) $\square \mathrm{Mstn}^{+/+(\mathrm{Het})}$}

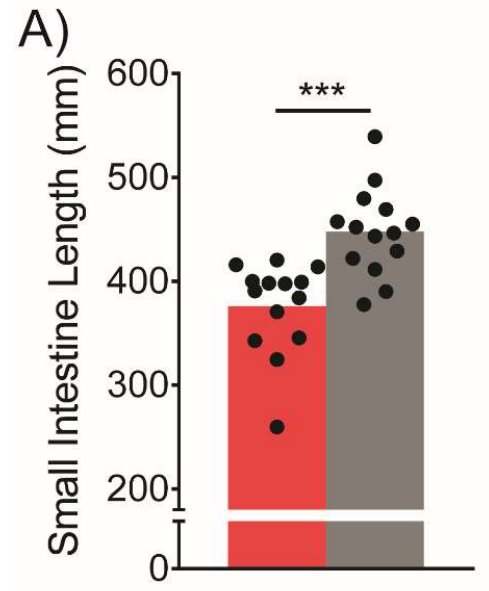

B)

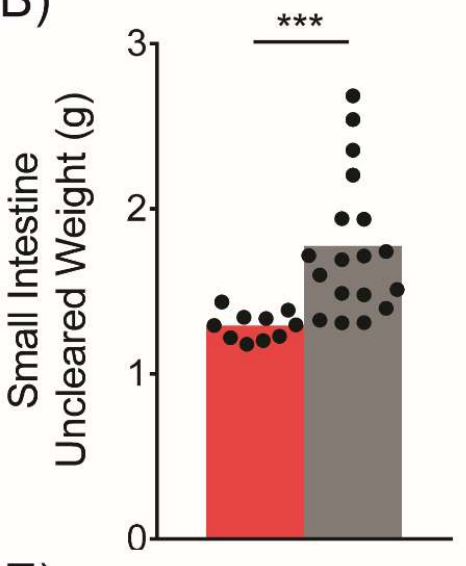

D)

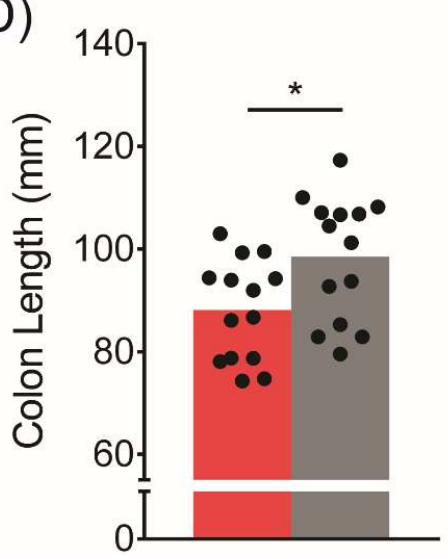

E)

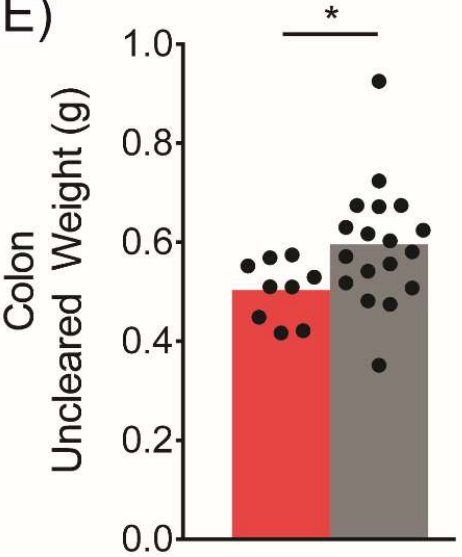

C)

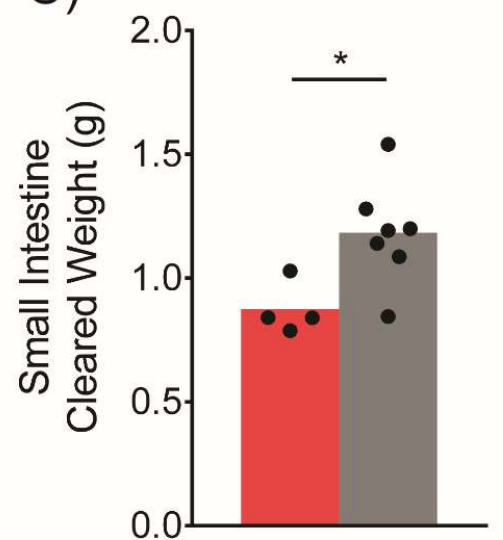

F)

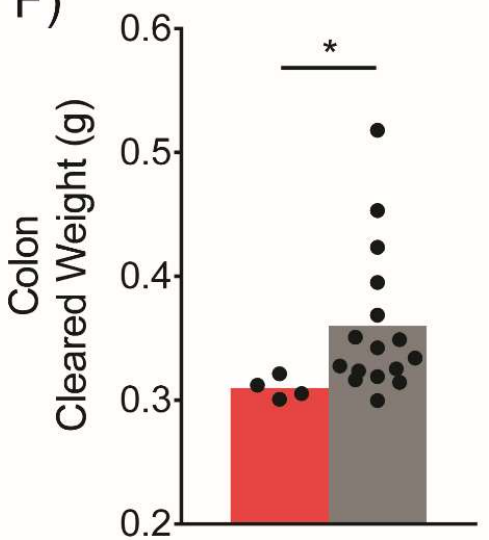

Figure 7.13.4 (Section 5.5): Colony control $\mathrm{Mstn}^{+/+}$and $\mathrm{Mstn}^{+/+}(\mathrm{Het})$ mice intestinal lengths and weights.

A) Small intestine length (mm) for colony control $\mathrm{Msth}^{+/+}(\mathrm{n}=14)$ and $\mathrm{Msth}^{+/+}(\mathrm{Het})(\mathrm{n}=14)$ mice. Mice were between 18 to 20 weeks old. Significance was assessed by Student's t-test with Welch correction: ${ }^{*} p<0.05, * * p<0.01, * * * p<0.001$.

B) Small intestine uncleared weight (grams) for colony control $\mathrm{Mstn}^{+/+}(\mathrm{n}=10)$ and $\mathrm{Msth}^{+/+(\mathrm{Het})}(\mathrm{n}=18)$ mice. Mice were between 18 to 21 weeks old. Significance was assessed by Student's t-test with Welch correction: $* p<0.05, * * p<0.01, * * * p<0.001$.

C) Small intestine cleared weight (grams) for colony control $\mathrm{Msth}^{+/+}(\mathrm{n}=4)$ and $\mathrm{Mstn}^{+/+}{ }^{(\mathrm{Het})}(\mathrm{n}=7)$ mice. Mice were between 18 to 21 weeks old. Significance was assessed by Student's t-test with Welch correction: ${ }^{*} p<0.05, * * p<0.01, * * * p<0.001$.

D) Colon length $(\mathrm{mm})$ for colony control $\mathrm{Mstn}^{+/+}(\mathrm{n}=14)$ and $\mathrm{Mstn}^{+/+}(\mathrm{Het})(\mathrm{n}=14)$ mice. Mice were between 18 to 20 weeks old. Significance was assessed by Student's t-test with Welch correction: ${ }^{*} p<0.05, * * p<0.01, * * * p<0.001$.

E) Colon uncleared weight (grams) for colony control $\mathrm{Mstn}^{+/+}(\mathrm{n}=9)$ and $\mathrm{Mstn}^{+/+}(\mathrm{Het})(\mathrm{n}=18)$ mice. Mice were between 18 to 21 weeks old. Significance was assessed by Student's t-test with Welch correction: ${ }^{*} p<0.05, * * p<0.01, * * * p<0.001$.

F) Colon cleared weight (grams) for colony control $\mathrm{Mstn}^{+/+}(\mathrm{n}=4)$ and $\mathrm{Mstn}^{+/+}(\mathrm{Het})(\mathrm{n}=16)$ mice. Mice were between 18 to 21 weeks old. Significance was assessed by Student's t-test with Welch correction: ${ }^{*} p<0.05, * * p<0.01, * * * p<0.001$.

Please note that the data for $\mathrm{Mstn}^{++(\mathrm{Het})}$ mice were also represented in Figure 4.11.1 as $\mathrm{Msth}^{+/+}$. 


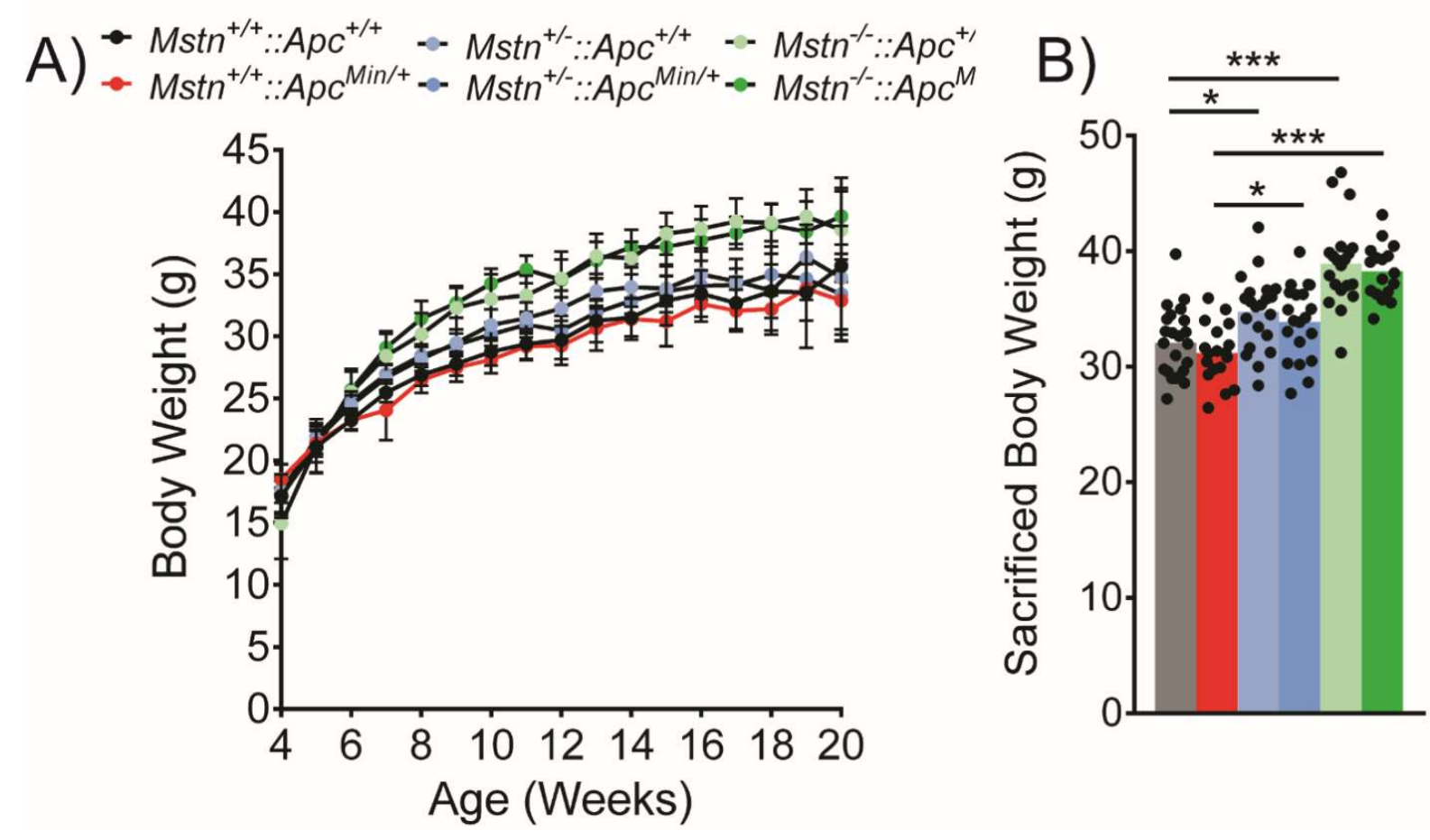

Figure 7.13.5 (Section 5.5): Growth curves and skeletal muscle weights of $\mathrm{Mstn}^{+/+}:: \mathrm{Apc}^{\mathrm{Min} /+}$, $\mathrm{Mstn}^{+/-}:: \mathrm{Apc}^{\mathrm{Min} /+}$, and $\mathrm{Mstn}^{-/}: \because \mathrm{Apc}^{\mathrm{Min} /+}$ mice and their respective controls.

A) Weekly body weights for male $M_{s t n}^{+/+}: \because A p c^{M i n /+}(\mathrm{n}=6-21), M_{s t n}^{+/} \because \because A p c^{M i n /+}(\mathrm{n}=8-34)$, and $M s t n^{-/}$ $\therefore A p c^{M i n /+}(\mathrm{n}=8-20)$ mice and their respective wild type controls, $M_{s t n^{+/+}}: \because A p c^{+/+}(\mathrm{n}=5-26), M_{s t n^{+/}}$ $\because: A p c^{+/+}(\mathrm{n}=10-38)$, and $M s n^{-/} \because \because A p c^{+/+}(\mathrm{n}=6-19)$ mice. Body weights are presented from 6 to 18 weeks. Error bars denote $\pm 95 \%$ CI.

B) Sacrificed body weights (grams) for $\operatorname{Mstn}^{+/+}: \because A p c^{M i n /+}$ (n=18), $\operatorname{Mstn}^{+/} \because: A p c^{M i n /+}(\mathrm{n}=21)$, and Mstn

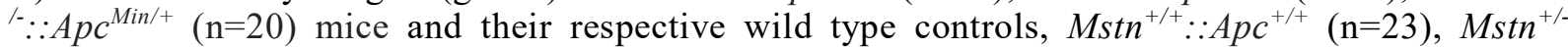
$\because A p c^{+/+}(\mathrm{n}=20)$, and $M_{s t n}{ }^{-/} \because A A c^{+/+}(\mathrm{n}=17)$ mice. Mice were between 18 to 22 weeks of age. The significance between the $A p c^{+/+}$and $A p c^{\operatorname{Min} /+}$ mice were assessed by Student's t-test with Welch correction: No significant difference was observed. The significance between $\mathrm{Mstn}^{+/+}:: A p c^{+/+}, \mathrm{Mstn}^{+/-}$ $\because A p c^{+/+}$, and $\mathrm{Mstn}^{-/}: \because A p c^{+/+}$mice was assessed by one-way ANOVA followed by Tukey's post-hoc test: ${ }^{*} p<0.05,{ }^{* *} p<0.01, * * * p<0.001$. Furthermore, the significance between $M_{s t n}{ }^{+/+}: \because A p c^{M i n /+}$, $M_{s t n^{+/}}{ }^{+}: A p c^{\mathrm{Min} /+}$, and $\mathrm{Mstn}^{-/}: \because A p c^{\mathrm{Min} /+}$ mice was assessed by one-way ANOVA followed by Tukey's post-hoc test: ${ }^{*} p<0.05,{ }^{* *} p<0.01,{ }^{* * *} p<0.001$. Please note that the data for $\operatorname{Mstn}^{+/+}: \because A p c^{+/+}(\mathrm{n}=23)$ and $\operatorname{Mstn}^{+/+}: \because A p c^{M i n /+}(\mathrm{n}=18)$ were also represented in Figure 7.13.3A and 7.13.3D as stn $^{+/+} \because: A p c^{+/+}$ (Het) and $\mathrm{Mstn}^{+++}: \because A p c^{\mathrm{Min} /+}(\mathrm{Het})$. 

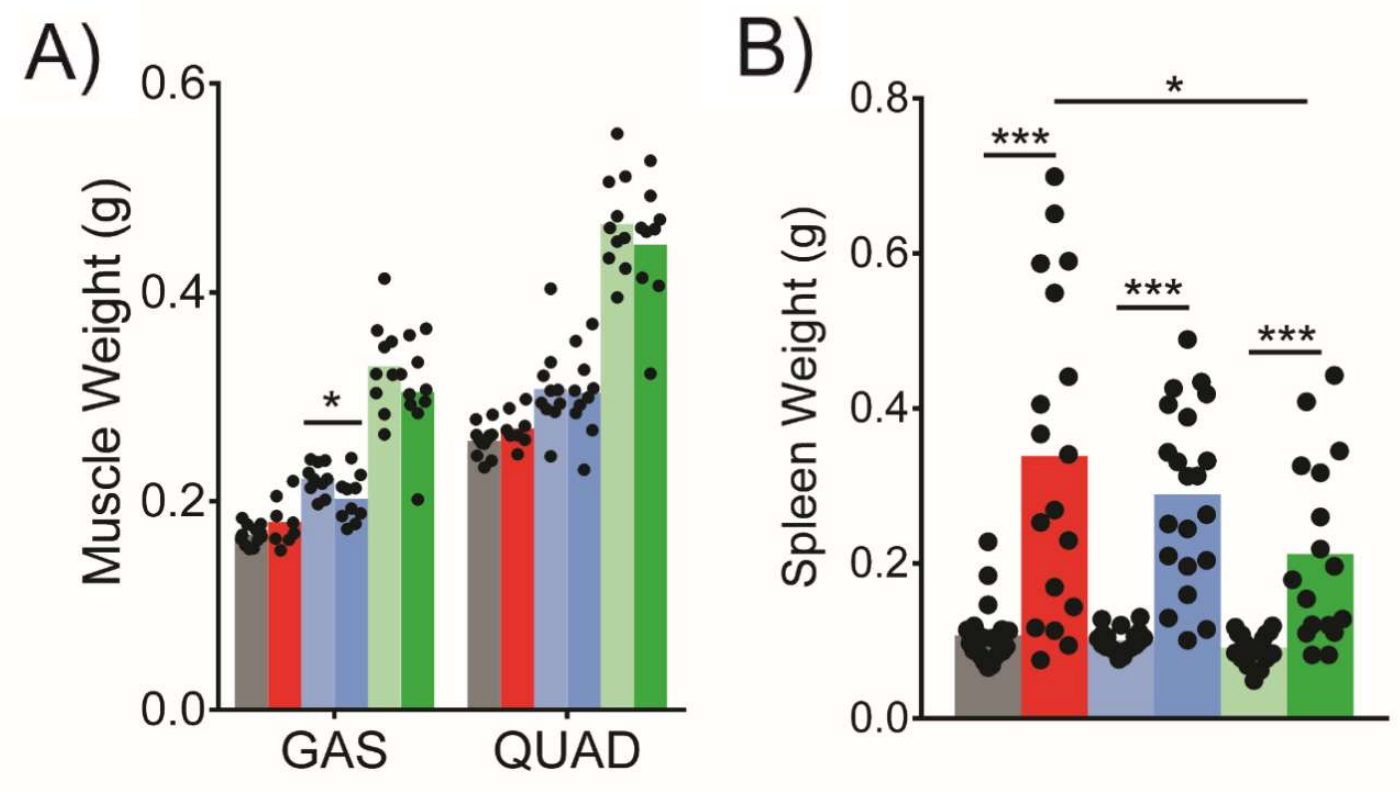

\begin{tabular}{|c|c|c|}
\hline $\mathrm{Mstn}^{+/+}: . \mathrm{Apc}^{+/+}$ & $\mathrm{Mstn}^{+/-}:: A p c^{+/+}$ & 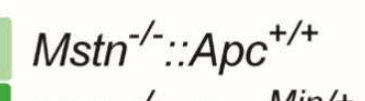 \\
\hline $\mathrm{Mstn}^{+/+}: . A p c^{\mathrm{Min} /+}$ & $M s t n^{+/-}: . A p c^{M i n /+}$ & $M s t n^{-/-}: . A p c^{M}$ \\
\hline
\end{tabular}

Figure 7.13.6 (Section 5.5): Growth curves and skeletal muscle weights of $\mathrm{Mstn}^{+/+}:: \mathrm{Apc}^{\mathrm{Min} /+}$, $M_{s t n}^{+/-}:: A p c^{M i n /+}$, and $M_{s t n}{ }^{-/}:: A p c^{M i n /+}$ mice and their respective controls.

A) Gastrocnemius (GAS) and quadricep (QUAD) weights (grams) for $\mathrm{Mstn}^{+/+}: \because A p c^{\mathrm{Min} /+}(\mathrm{n}=8), \mathrm{Mstn}^{+/-}$ $\because A p c^{M i n /+}(\mathrm{n}=10)$, and $\operatorname{Mstn}^{-/} \because \because A p c^{M i n /+}(\mathrm{n}=9)$ mice and their respective wild type controls, $\operatorname{Mstn}^{+/+}: \because A p c^{+/+}(\mathrm{n}=12), M_{s t n}^{+/} \because: A p c^{+/+}(\mathrm{n}=10)$, and $\operatorname{Mstn}^{-/}: \because A p c^{+/+}(\mathrm{n}=10)$ mice. Mice were between 18 to 21 weeks of age. The significance between $\mathrm{Apc}^{+/+}$and $\mathrm{Apc}^{\mathrm{Min} /+}$ mice were assessed by Student's t-test with Welch correction: $* p<0.05, * * p<0.01, * * * p<0.001$. The significance between $\mathrm{Mstn}^{+/+}: \because \mathrm{Apc}^{\mathrm{Min/+}}, \mathrm{Mstn}^{+/} \because: A p c^{\mathrm{Min} /+}$, and $\mathrm{Mstn}^{-/} \because: \mathrm{Apc}^{\mathrm{Min} /+}$ mice, and the significance between

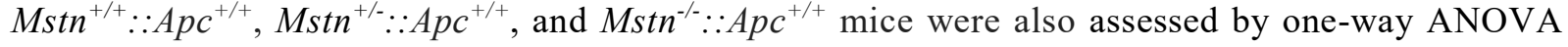
followed by Tukey's post-hoc test. The results are not displayed on this graph. The information is represented in Table 7.13.5. Please note that the data for $\operatorname{Mstn}^{+++}: \because \mathrm{Apc}^{+/+}(\mathrm{n}=12)$ and $\mathrm{Mstn}^{+/+}: \because A p c^{\mathrm{Min} /+}$ $(\mathrm{n}=8)$ were also represented in Figure $7.13 .3 \mathrm{~B}$ and $7.13 .3 \mathrm{E}$ as $\mathrm{Mstn}^{+/+}: \because A p c^{+/+}$(Het) and $\mathrm{Msth}^{+/+}: \because A p c^{\mathrm{Min} /+(\mathrm{Het})}$.

B) Spleen weights (grams) for $\operatorname{Mstn}^{+/+}: \because A p c^{M i n /+}(\mathrm{n}=18), M_{s t n^{+/}-}: A p c^{M i n /+}(\mathrm{n}=21)$, and $M_{s t n^{-/-}}$ $\because A p c^{M i n /+}(\mathrm{n}=17)$ mice and their respective wild type controls, $M_{s t n^{+/+}}: A p c^{+/+}(\mathrm{n}=22), M_{s t n^{+/-}}: A p c^{+/+}$ $(\mathrm{n}=19)$, and $M_{s t n}{ }^{-/}: \because A p c^{+/+}(\mathrm{n}=20)$ mice. Mice were between 18 to 22 weeks of age. The significance between $\mathrm{Apc}^{\mathrm{Min} /+}$ and $\mathrm{Apc}^{+/+}$mice was assessed by Student's t-test with Welch correction: ${ }^{*} p<0.05$, ${ }^{* *} p<0.01, * * * p<0.001$. Significance between $\mathrm{Mstn}^{+/+}: \because A p c^{\mathrm{Min} /+}, \mathrm{Mstn}^{+/} \because \because A p c^{\mathrm{Min} /+}$, and $\mathrm{Msth}^{-1-}$ $\because A p c^{\mathrm{Min} /+}$ mice was assessed by one-way ANOVA followed by Tukey's post-hoc test: ${ }^{*} p<0.05$, ${ }^{* *} p<0.01,{ }^{* * *} p<0.001$. Please note that the data for $M_{s t n^{+/+}}: A p c^{+/+}(\mathrm{n}=22)$ and $M_{s i n} n^{++}: \because A p c^{\text {Min } /+}$ $(\mathrm{n}=18)$ were also represented in Figure $7.13 .3 \mathrm{C}$ and $7.13 .3 \mathrm{~F}$ as $\mathrm{Mstn}^{+/+}: \because \mathrm{Apc}^{+/+}{ }^{\text {(Het) }}$ and $\mathrm{Mstn}^{+/+}:: A p c^{\mathrm{Min} /+(\mathrm{Het})}$. 
Table 7.13.5 (Section 5.5, Figure 7.13.6A): Skeletal muscle weights for $\mathrm{Mstn}^{+/+}:: \mathrm{Apc}^{\mathrm{Min} /+}$ $(\mathrm{n}=8), \mathrm{Mstn}^{+/-}:: \mathrm{Apc}^{\mathrm{Min} /+}(\mathrm{n}=10)$, and $\mathrm{Mstn}^{-/-}:: \mathrm{Apc}^{\mathrm{Min} /+}(\mathrm{n}=9)$ mice and their respective wild

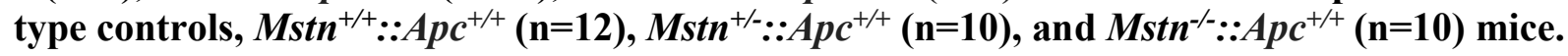

\begin{tabular}{lcccccc} 
& \multicolumn{2}{c}{$\mathrm{Mstn}^{+/+}$} & \multicolumn{2}{c}{$\mathrm{Mstn}^{+/}$} & \multicolumn{2}{c}{$\mathrm{Msn}^{-/}$} \\
\cline { 2 - 7 } & $A p c^{+/+}$ & $A p c^{\mathrm{Min} /+}$ & $A p c^{+/+}$ & $A p c^{\mathrm{Min} /+}$ & $A p c^{+/+}$ & $A p c^{\mathrm{Min} /+}$ \\
\hline TA weight $(\mathrm{g})$ & 0.0511 & 0.0519 & $0.0619 \dagger$ & 0.0594 & $0.0916 \bullet \bullet$ & $0.0898^{* * *}$ \\
\pm S.D. & 0.0051 & 0.0053 & 0.0054 & 0.0053 & 0.0123 & 0.0131 \\
\hline EDL weight (g) & 0.0089 & 0.0107 & $0.0136 \bullet$ & 0.0126 & $0.0195 \bullet \bullet$ & $0.0176^{* * *}$ \\
\pm S.D. & 0.0022 & 0.0038 & 0.0016 & 0.0016 & 0.0054 & 0.0031 \\
\hline GAS weight (g) & 0.1675 & 0.1798 & $0.2214 \bullet$ & $0.2023 \dagger$ & $0.3293 \bullet \bullet$ & $0.3045^{* * *}$ \\
\pm S.D. & 0.0094 & 0.0226 & 0.0151 & 0.0219 & 0.0426 & 0.0484 \\
\hline SOL weight (g) & 0.0072 & 0.0067 & 0.0076 & 0.0077 & $0.0116 \bullet \bullet$ & $0.0108^{* * *}$ \\
\pm S.D. & 0.0015 & 0.0009 & 0.0010 & 0.0020 & 0.019 & 0.0023 \\
\hline QUAD weight & & & & & & \\
g) & 0.2579 & 0.2694 & $0.3073 \bullet$ & 0.3037 & $0.4655 \bullet \bullet$ & $0.4457^{* * *}$ \\
\pm S.D. & 0.0147 & 0.0169 & 0.0414 & 0.0402 & 0.0466 & 0.0588 \\
\hline
\end{tabular}

$\dagger$ Significantly different to respective wild type skeletal muscle weight controls $(\mathrm{p}<0.05)$

Significance was assessed by Student's t-test with Welch correction

\$ Significantly different to $\mathrm{Mstn}^{+/+}: \because A p c^{+/+}$skeletal muscle weight $(\mathrm{p}<0.05)$

- Significantly different to $M s t n^{+/+}: \because A p c^{+/+}$skeletal muscle weight $(\mathrm{p}<0.01)$

-• Significantly different to $M s n^{+/+}: \because A p c^{M i n /+}$ skeletal muscle weight $(\mathrm{p}<0.001)$

Significance was assessed by one-way ANOVA followed by Tukey's post-hoc test

* Significantly different to $M s t n^{+/+}:: A p c^{\text {Min } /+}$ skeletal muscle weight $(\mathrm{p}<0.05)$

** Significantly different to $M s n^{+/+} \because: A p c^{M i n /+}$ skeletal muscle weight $(\mathrm{p}<0.01)$

*** Significantly different to $\mathrm{Mstn}^{+/+}: \because A p c^{\text {Min/+ }}$ skeletal muscle weight $(\mathrm{p}<0.001)$

Significance was assessed by one-way ANOVA followed by Tukey's post-hoc test 
Table 7.13.6 (Section 5.5, Figures 7.13.5 and 7.13.6): Tibia lengths for $\mathrm{Mstn}^{+/+}:: \mathrm{Apc}^{\mathrm{Min} /+}$ $(\mathrm{n}=9), \mathrm{Mstn}^{+/-}: \mathrm{Apc}^{\mathrm{Min} /+}(\mathrm{n}=13)$, and $\mathrm{Mstn}^{-/}:: \mathrm{Apc}^{\mathrm{Min} /+}(\mathrm{n}=9)$ mice and their respective wild

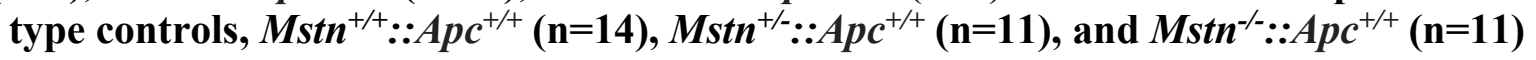
mice.

\begin{tabular}{lcccccc} 
& \multicolumn{2}{c}{$\mathrm{Mstn}^{+/+}$} & \multicolumn{2}{c}{$\mathrm{Mstn}^{+/}$} & \multicolumn{2}{c}{$\mathrm{Msth}^{-/-}$} \\
\cline { 2 - 7 } & $A p c^{+/+}$ & $A p c^{\mathrm{Min} /+}$ & $A p c^{+/+}$ & $A p c^{\mathrm{Min} /+}$ & $A p c^{+/+}$ & $A p c^{\mathrm{Min} /+}$ \\
\hline Tibia Length & 18.28 & 18.07 & 18.43 & $18.45 \dagger$ & 18.41 & $18.55 \ddagger$ \\
(mm) & 0.37 & 0.23 & 0.21 & 0.23 & 0.21 & 0.25 \\
\pm S.D. & & & & & & \\
\hline
\end{tabular}

$\uparrow$ Significantly different to $\mathrm{Mstn}^{+/+}: \because A p c^{\mathrm{Min} /+}$ tibia length $(\mathrm{p}<0.01)$

$\$$ Significantly different to $M s n^{+/+}: \because A p c^{M i n /+}$ tibia length $(\mathrm{p}<0.001)$

Significance was assessed by one-way ANOVA followed by Tukey's post-hoc test 


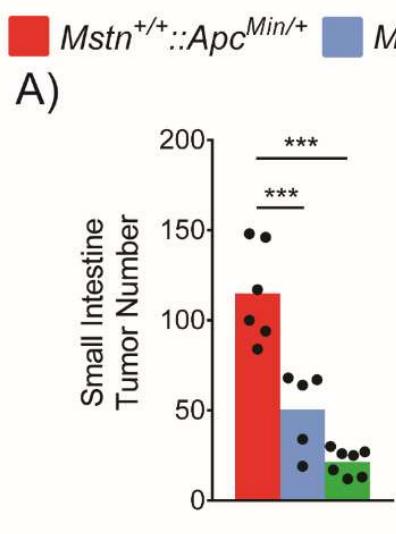

C)
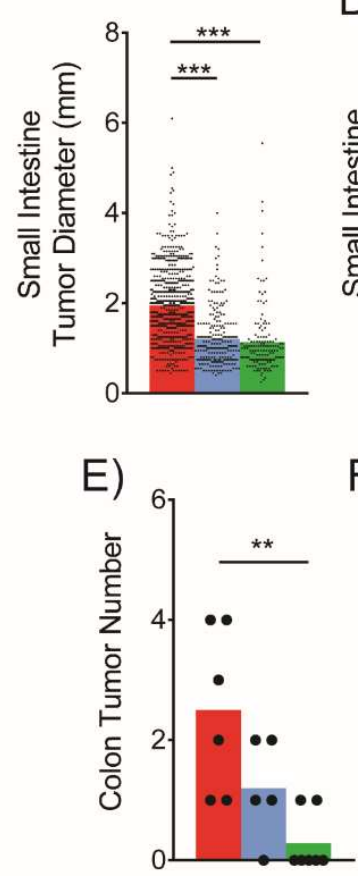

$\mathrm{Mstn}^{+/ \ldots}:: \mathrm{ApC}^{\mathrm{Min} /+} \square \mathrm{Mstn}^{-/}: \because \mathrm{Apc}^{\mathrm{Min} /+}$

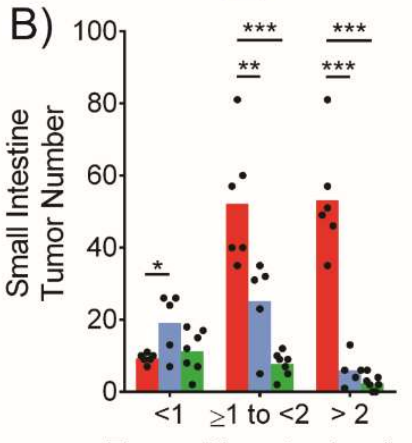

Tumor Diameter $(\mathrm{mm})$

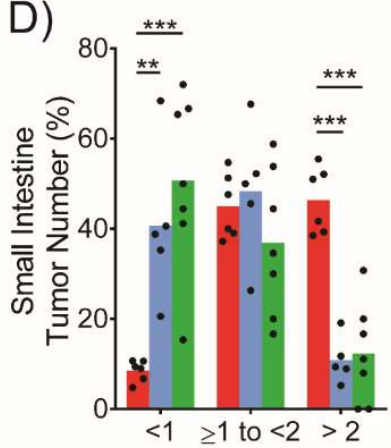

F)

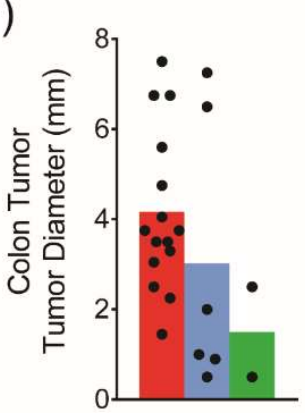

Figure 7.13.7 (Section 5.5): The deletion of myostatin suppressed the intestinal tumor number and diameter in 18 week old $A p c^{\mathrm{Min} /+}$ mice.

A) Small intestine tumor number and B) Small intestine tumor diameter $(\mathrm{mm})$ for $\operatorname{Mstn}^{+/+}: \because A_{p c} c^{M i n /+}(\mathrm{n}=6), M_{s t n}^{+/-} \because: A p c^{M i n /+}(\mathrm{n}=5)$, and $\operatorname{Mstn}^{-/}: \because A p c^{M i n /+}(\mathrm{n}=7)$. Significance was assessed by one-way ANOVA followed by Tukey's post-hoc test: ${ }^{*} p<0.05,{ }^{* *} p<0.01$, $* * * p<0.001$.

C) Average small intestine tumor diameter (mm) for $\operatorname{Mstn}^{+/+}: \because A p c^{M i n /+}(\mathrm{n}=6), M_{s t n^{+/}}: \because A p c^{M i n /+}$ $(\mathrm{n}=5)$, and $\mathrm{Mstn}^{-/} \because \because A p c^{\mathrm{Min} /+}(\mathrm{n}=7)$. While $\mathrm{n}$ represents the number of mice used in this experiment, one data point represents the diameter of a single small intestinal tumor. Significance was assessed by one-way ANOVA followed by Tukey's post-hoc test: * $p<0.05, * * p<0.01,{ }^{* * *} p<0.001$.

D) Small intestine tumor diameter (\%) for $\operatorname{Mstn}^{+/+}: \because A p c^{M i n /+}(\mathrm{n}=6), \operatorname{Mstn}^{+/-} \because: A p c^{M i n /+}(\mathrm{n}=5)$, and $M \operatorname{stn}^{-/}: \because A p c^{M i n /+}(\mathrm{n}=7)$. Each data point represents the number of tumors that belong to the stated diameter over the total number of tumors per animal. Data was represented as a percentage. Significance was assessed by one-way ANOVA followed by Tukey's post-hoc test: ${ }^{*} p<0.05$, $* * p<0.01, * * * p<0.001$

E) Colon tumor number and F) Colon tumor diameter (mm) for $\mathrm{Mstn}^{+/+}:: A p c^{\mathrm{Min} /+}(\mathrm{n}=6), \mathrm{Mstn}^{+/}$ $\because A p c^{M i n /+}(\mathrm{n}=5)$, and $M s t n^{-/}: \because A p c^{M i n /+}(\mathrm{n}=7)$. For Figure 7.13.7F, $\mathrm{n}$ represents the number of mice used for this experiment, but each data point represents the diameter of a single colon tumor. Significance was assessed by one-way ANOVA followed by Tukey's post-hoc test: ${ }^{*} p<0.05$, $* * p<0.01, * * * p<0.001$. 


\section{References}

1. Jenkins GW, Kemnitz CP, Tortora GJ. Anatomy and Physiology: From Science to Life. 2nd ed: John Wiley \& Sons, Inc.; 2006.

2. Pedersen BK, Febbraio MA. Muscle as an endocrine organ: focus on muscle-derived interleukin-6. Physiological reviews. 2008;88(4):1379-406. doi:

10.1152/physrev.90100.2007. PubMed PMID: 18923185.

3. Hill JA, Olson EN. Muscle: Fundamental Biology and Mechanisms of Disease. Elsevier Science; 2012.

4. Charge SB, Rudnicki MA. Cellular and molecular regulation of muscle regeneration. Physiological reviews. 2004;84(1):209-38. doi: 10.1152/physrev.00019.2003. PubMed PMID: 14715915.

5. Glass DJ. Skeletal muscle hypertrophy and atrophy signaling pathways. The international journal of biochemistry \& cell biology. 2005;37(10):1974-84. doi: 10.1016/j.biocel.2005.04.018. PubMed PMID: 16087388.

6. Tisdale MJ. Mechanisms of cancer cachexia. Physiological reviews. 2009;89(2):381410. doi: 10.1152/physrev.00016.2008. PubMed PMID: 19342610.

7. Barber MD, Wigmore SJ, Ross JA, Fearon KC, Tisdale MJ. Proinflammatory cytokines, nutritional support, and the cachexia syndrome: interactions and therapeutic options. Cancer. 1998;82(5):1000. PubMed PMID: 9486598.

8. Warrick HM, Spudich JA. Myosin structure and function in cell motility. Annual review of cell biology. 1987;3:379-421. doi: 10.1146/annurev.cb.03.110187.002115. PubMed PMID: 3318880.

9. Rayment I, Rypniewski WR, Schmidt-Base K, Smith R, Tomchick DR, Benning MM, et al. Three-dimensional structure of myosin subfragment-1: a molecular motor. Science. 1993;261(5117):50-8. PubMed PMID: 8316857.

10. Huxley HE. Electron Microscope Studies on the Structure of Natural and Synthetic Protein Filaments from Striated Muscle. Journal of molecular biology. 1963;7:281-308. PubMed PMID: 14064165.

11. Nobes CD, Hall A. Rho, rac and cdc42 GTPases: regulators of actin structures, cell adhesion and motility. Biochemical Society transactions. 1995;23(3):456-9. PubMed PMID: 8566347.

12. Rayment I, Holden HM, Whittaker M, Yohn CB, Lorenz M, Holmes KC, et al. Structure of the actin-myosin complex and its implications for muscle contraction. Science. 1993;261(5117):58-65. PubMed PMID: 8316858.

13. Spudich JA, Watt S. The regulation of rabbit skeletal muscle contraction. I.

Biochemical studies of the interaction of the tropomyosin-troponin complex with actin and the proteolytic fragments of myosin. The Journal of biological chemistry. 1971;246(15):4866-71. PubMed PMID: 4254541.

14. Knappeis GG, Carlsen F. The ultrastructure of the $\mathrm{Z}$ disc in skeletal muscle. The Journal of cell biology. 1962;13:323-35. PubMed PMID: 14457201; PubMed Central PMCID: PMC2106831.

15. Carlsen F, Knappeis GG. The fine structure of the $\mathrm{Z}$ disc in skeletal muscle revealed by electron microscopy. Danish medical bulletin. 1962;9:18. PubMed PMID: 13876627.

16. Obermann WM, Gautel M, Weber K, Furst DO. Molecular structure of the sarcomeric $\mathrm{M}$ band: mapping of titin and myosin binding domains in myomesin and the identification of a potential regulatory phosphorylation site in myomesin. The EMBO journal. 
1997;16(2):211-20. doi: 10.1093/emboj/16.2.211. PubMed PMID: 9029142; PubMed Central PMCID: PMC1169628.

17. Tskhovrebova L, Trinick J, Sleep JA, Simmons RM. Elasticity and unfolding of single molecules of the giant muscle protein titin. Nature. 1997;387(6630):308-12. doi: 10.1038/387308a0. PubMed PMID: 9153398.

18. Labeit S, Kolmerer B. Titins: giant proteins in charge of muscle ultrastructure and elasticity. Science. 1995;270(5234):293-6. PubMed PMID: 7569978.

19. Huxley AF, Niedergerke R. Structural changes in muscle during contraction; interference microscopy of living muscle fibres. Nature. 1954;173(4412):971-3. PubMed PMID: 13165697.

20. Endo M. Calcium release from the sarcoplasmic reticulum. Physiological reviews. 1977;57(1):71-108. PubMed PMID: 13441.

21. Gage PW, Eisenberg RS. Action potentials, afterpotentials, and excitation-contraction coupling in frog sartorius fibers without transverse tubules. The Journal of general physiology. 1969;53(3):298-310. PubMed PMID: 5767334; PubMed Central PMCID: PMC2202907.

22. Aukland K, Reed RK. Interstitial-lymphatic mechanisms in the control of extracellular fluid volume. Physiological reviews. 1993;73(1):1-78. PubMed PMID: 8419962.

23. Wrann CD, White JP, Salogiannnis J, Laznik-Bogoslavski D, Wu J, Ma D, et al. Exercise induces hippocampal BDNF through a PGC-1alpha/FNDC5 pathway. Cell metabolism. 2013;18(5):649-59. doi: 10.1016/j.cmet.2013.09.008. PubMed PMID: 24120943.

24. Bostrom P, Wu J, Jedrychowski MP, Korde A, Ye L, Lo JC, et al. A PGC1-alphadependent myokine that drives brown-fat-like development of white fat and thermogenesis. Nature. 2012;481(7382):463-8. doi: 10.1038/nature10777. PubMed PMID: 22237023; PubMed Central PMCID: PMC3522098.

25. Seale P, Sabourin LA, Girgis-Gabardo A, Mansouri A, Gruss P, Rudnicki MA. Pax7 is required for the specification of myogenic satellite cells. Cell. 2000;102(6):777-86. PubMed PMID: 11030621.

26. Wang YX, Zhang CL, Yu RT, Cho HK, Nelson MC, Bayuga-Ocampo CR, et al. Regulation of muscle fiber type and running endurance by PPARdelta. PLoS biology. 2004;2(10):e294. doi: 10.1371/journal.pbio.0020294. PubMed PMID: 15328533; PubMed Central PMCID: PMC509410.

27. Schiaffino S, Reggiani C. Molecular diversity of myofibrillar proteins: gene regulation and functional significance. Physiological reviews. 1996;76(2):371-423. PubMed PMID: 8618961.

28. Bassel-Duby R, Olson EN. Signaling pathways in skeletal muscle remodeling. Annual review of biochemistry. 2006;75:19-37. doi: 10.1146/annurev.biochem.75.103004.142622. PubMed PMID: 16756483.

29. Schiaffino S, Dyar KA, Ciciliot S, Blaauw B, Sandri M. Mechanisms regulating skeletal muscle growth and atrophy. FEBS J. 2013;280(17):4294-314. doi:

10.1111/febs.12253. PubMed PMID: 23517348.

30. Evans WJ, Morley JE, Argiles J, Bales C, Baracos V, Guttridge D, et al. Cachexia: a new definition. Clinical nutrition. 2008;27(6):793-9. doi: 10.1016/j.clnu.2008.06.013. PubMed PMID: 18718696.

31. Pette D, Staron RS. Transitions of muscle fiber phenotypic profiles. Histochemistry and cell biology. 2001;115(5):359-72. PubMed PMID: 11449884. 
32. Ciciliot S, Rossi AC, Dyar KA, Blaauw B, Schiaffino S. Muscle type and fiber type specificity in muscle wasting. The international journal of biochemistry \& cell biology. 2013;45(10):2191-9. doi: 10.1016/j.biocel.2013.05.016. PubMed PMID: 23702032.

33. Park SW, Goodpaster BH, Lee JS, Kuller LH, Boudreau R, de Rekeneire N, et al. Excessive loss of skeletal muscle mass in older adults with type 2 diabetes. Diabetes care. 2009;32(11):1993-7. doi: 10.2337/dc09-0264. PubMed PMID: 19549734; PubMed Central PMCID: PMC2768193.

34. Grinspoon S, Mulligan K, Department of H, Human Services Working Group on the $\mathrm{P}$, Treatment of $\mathrm{W}$, Weight $\mathrm{L}$. Weight loss and wasting in patients infected with human immunodeficiency virus. Clinical infectious diseases : an official publication of the Infectious Diseases Society of America. 2003;36(Suppl 2):S69-78. doi: 10.1086/367561. PubMed PMID: 12652374.

35. Tiao G, Hobler S, Wang JJ, Meyer TA, Luchette FA, Fischer JE, et al. Sepsis is associated with increased mRNAs of the ubiquitin-proteasome proteolytic pathway in human skeletal muscle. The Journal of clinical investigation. 1997;99(2):163-8. doi:

10.1172/JCI1 19143. PubMed PMID: 9005983; PubMed Central PMCID: PMC507782.

36. Fearon K, Strasser F, Anker SD, Bosaeus I, Bruera E, Fainsinger RL, et al. Definition and classification of cancer cachexia: an international consensus. The lancet oncology. 2011;12(5):489-95. doi: 10.1016/S1470-2045(10)70218-7. PubMed PMID: 21296615. 37. Kritchevsky SB, Wilcosky TC, Morris DL, Truong KN, Tyroler HA. Changes in plasma lipid and lipoprotein cholesterol and weight prior to the diagnosis of cancer. Cancer research. 1991;51(12):3198-203. PubMed PMID: 2039996.

38. Dewys WD, Begg C, Lavin PT, Band PR, Bennett JM, Bertino JR, et al. Prognostic effect of weight loss prior to chemotherapy in cancer patients. Eastern Cooperative Oncology Group. The American journal of medicine. 1980;69(4):491-7. PubMed PMID: 7424938.

39. DeWys WD. Weight loss and nutritional abnormalilties in cancer patients: incidence, severity, and significance1986.

40. Schmitt TL, Martignoni ME, Bachmann J, Fechtner K, Friess H, Kinscherf R, et al. Activity of the Akt-dependent anabolic and catabolic pathways in muscle and liver samples in cancer-related cachexia. J Mol Med (Berl). 2007;85(6):647-54. doi: 10.1007/s00109-0070177-2. PubMed PMID: 17333095.

41. Zampieri S, Doria A, Adami N, Biral D, Vecchiato M, Savastano S, et al. Subclinical myopathy in patients affected with newly diagnosed colorectal cancer at clinical onset of disease: evidence from skeletal muscle biopsies. Neurol Res. 2010;32(1):20-5. doi: 10.1179/016164110X12556180205997. PubMed PMID: 19941733.

42. Weber MA, Krakowski-Roosen H, Schroder L, Kinscherf R, Krix M, Kopp-Schneider A, et al. Morphology, metabolism, microcirculation, and strength of skeletal muscles in cancer-related cachexia. Acta Oncol. 2009;48(1):116-24. doi: 10.1080/02841860802130001. PubMed PMID: 18607877.

43. Eley HL, Skipworth RJ, Deans DA, Fearon KC, Tisdale MJ. Increased expression of phosphorylated forms of RNA-dependent protein kinase and eukaryotic initiation factor 2alpha may signal skeletal muscle atrophy in weight-losing cancer patients. British journal of cancer. 2008;98(2):443-9. doi: 10.1038/sj.bjc.6604150. PubMed PMID: 18087277; PubMed Central PMCID: PMCPMC2361431.

44. Kilgour RD, Vigano A, Trutschnigg B, Hornby L, Lucar E, Bacon SL, et al. Cancerrelated fatigue: the impact of skeletal muscle mass and strength in patients with advanced cancer. Journal of cachexia, sarcopenia and muscle. 2010;1(2):177-85. doi: 10.1007/s13539010-0016-0. PubMed PMID: 21475694; PubMed Central PMCID: PMCPMC3060645. 
45. Bachmann J, Buchler MW, Friess H, Martignoni ME. Cachexia in patients with chronic pancreatitis and pancreatic cancer: impact on survival and outcome. Nutrition and cancer. 2013;65(6):827-33. doi: 10.1080/01635581.2013.804580. PubMed PMID: 23909726. 46. Andreyev HJ, Norman AR, Oates J, Cunningham D. Why do patients with weight loss have a worse outcome when undergoing chemotherapy for gastrointestinal malignancies? European journal of cancer. 1998;34(4):503-9. PubMed PMID: 9713300.

47. Ross PJ, Ashley S, Norton A, Priest K, Waters JS, Eisen T, et al. Do patients with weight loss have a worse outcome when undergoing chemotherapy for lung cancers? British journal of cancer. 2004;90(10):1905-11. doi: 10.1038/sj.bjc.6601781. PubMed PMID: 15138470; PubMed Central PMCID: PMC2409471.

48. Glaus A. Fatigue and cachexia in cancer patients. Supportive care in cancer : official journal of the Multinational Association of Supportive Care in Cancer. 1998;6(2):77-8. PubMed PMID: 9540162.

49. Roberts BM, Frye GS, Ahn B, Ferreira LF, Judge AR. Cancer cachexia decreases specific force and accelerates fatigue in limb muscle. Biochemical and biophysical research communications. 2013;435(3):488-92. doi: 10.1016/j.bbrc.2013.05.018. PubMed PMID: 23673294; PubMed Central PMCID: PMC3708303.

50. Stewart GD, Skipworth RJ, Fearon KC. Cancer cachexia and fatigue. Clinical medicine. 2006;6(2):140-3. PubMed PMID: 16688969.

51. Mitch WE, Goldberg AL. Mechanisms of muscle wasting. The role of the ubiquitinproteasome pathway. The New England journal of medicine. 1996;335(25):1897-905. doi: 10.1056/NEJM199612193352507. PubMed PMID: 8948566.

52. Baracos VE. Cancer-associated cachexia and underlying biological mechanisms. Annual review of nutrition. 2006;26:435-61. doi: 10.1146/annurev.nutr.26.061505.111151. PubMed PMID: 16602932.

53. Laviano A, Cascino A, Muscaritoli M, Fanfarillo F, Rossi Fanelli F. Tumor-induced changes in host metabolism: a possible role for free tryptophan as a marker of neoplastic disease. Advances in experimental medicine and biology. 2003;527:363-6. PubMed PMID: 15206751 .

54. Emery PW, Edwards RH, Rennie MJ, Souhami RL, Halliday D. Protein synthesis in muscle measured in vivo in cachectic patients with cancer. British medical journal. 1984;289(6445):584-6. PubMed PMID: 6087973; PubMed Central PMCID: PMC1442890. 55. O'Keefe SJ, Ogden J, Ramjee G, Rund J. Contribution of elevated protein turnover and anorexia to cachexia in patients with hepatocellular carcinoma. Cancer research. 1990;50(4):1226-30. PubMed PMID: 2153453.

56. Schwartz AL, Ciechanover A. The ubiquitin-proteasome pathway and pathogenesis of human diseases. Annual review of medicine. 1999;50:57-74. doi:

10.1146/annurev.med.50.1.57. PubMed PMID: 10073263.

57. Glickman MH, Ciechanover A. The ubiquitin-proteasome proteolytic pathway: destruction for the sake of construction. Physiological reviews. 2002;82(2):373-428. doi: 10.1152/physrev.00027.2001. PubMed PMID: 11917093.

58. Ciechanover A. The ubiquitin-proteasome proteolytic pathway. Cell. 1994;79(1):1321. PubMed PMID: 7923371.

59. Cohen S, Brault JJ, Gygi SP, Glass DJ, Valenzuela DM, Gartner C, et al. During muscle atrophy, thick, but not thin, filament components are degraded by MuRF1-dependent ubiquitylation. The Journal of cell biology. 2009;185(6):1083-95. doi:

10.1083/jcb.200901052. PubMed PMID: 19506036; PubMed Central PMCID: PMC2711608. 60. Acharyya S, Ladner KJ, Nelsen LL, Damrauer J, Reiser PJ, Swoap S, et al. Cancer cachexia is regulated by selective targeting of skeletal muscle gene products. The Journal of 
clinical investigation. 2004;114(3):370-8. doi: 10.1172/JCI20174. PubMed PMID: 15286803; PubMed Central PMCID: PMCPMC484974.

61. Kwak KS, Zhou X, Solomon V, Baracos VE, Davis J, Bannon AW, et al. Regulation of protein catabolism by muscle-specific and cytokine-inducible ubiquitin ligase E3alpha-II during cancer cachexia. Cancer research. 2004;64(22):8193-8. doi: 10.1158/0008-5472.CAN04-2102. PubMed PMID: 15548684.

62. Lecker SH, Jagoe RT, Gilbert A, Gomes M, Baracos V, Bailey J, et al. Multiple types of skeletal muscle atrophy involve a common program of changes in gene expression.

FASEB journal : official publication of the Federation of American Societies for Experimental Biology. 2004;18(1):39-51. doi: 10.1096/fj.03-0610com. PubMed PMID: 14718385.

63. Zhou X, Wang JL, Lu J, Song Y, Kwak KS, Jiao Q, et al. Reversal of cancer cachexia and muscle wasting by ActRIIB antagonism leads to prolonged survival. Cell. 2010;142(4):531-43. doi: 10.1016/j.cell.2010.07.011. PubMed PMID: 20723755.

64. Yuan L, Han J, Meng Q, Xi Q, Zhuang Q, Jiang Y, et al. Muscle-specific E3 ubiquitin ligases are involved in muscle atrophy of cancer cachexia: an in vitro and in vivo study. Oncology reports. 2015;33(5):2261-8. doi: 10.3892/or.2015.3845. PubMed PMID: 25760630. 65. Bossola M, Muscaritoli M, Costelli P, Grieco G, Bonelli G, Pacelli F, et al. Increased muscle proteasome activity correlates with disease severity in gastric cancer patients. Annals of surgery. 2003;237(3):384-9. doi: 10.1097/01.SLA.0000055225.96357.71. PubMed PMID: 12616123; PubMed Central PMCID: PMCPMC1514311.

66. Stephens NA, Skipworth RJ, Gallagher IJ, Greig CA, Guttridge DC, Ross JA, et al. Evaluating potential biomarkers of cachexia and survival in skeletal muscle of upper gastrointestinal cancer patients. Journal of cachexia, sarcopenia and muscle. 2015;6(1):53-61. doi: 10.1002/jcsm.12005. PubMed PMID: 26136412; PubMed Central PMCID: PMCPMC4435097.

67. Bodine SC, Latres E, Baumhueter S, Lai VK, Nunez L, Clarke BA, et al. Identification of ubiquitin ligases required for skeletal muscle atrophy. Science. 2001;294(5547):1704-8. doi: 10.1126/science.1065874. PubMed PMID: 11679633.

68. Gomes AV, Waddell DS, Siu R, Stein M, Dewey S, Furlow JD, et al. Upregulation of proteasome activity in muscle RING finger 1-null mice following denervation. FASEB journal : official publication of the Federation of American Societies for Experimental Biology. 2012;26(7):2986-99. doi: 10.1096/fj.12-204495. PubMed PMID: 22508689; PubMed Central PMCID: PMC3382096.

69. Clarke BA, Drujan D, Willis MS, Murphy LO, Corpina RA, Burova E, et al. The E3 Ligase MuRF1 degrades myosin heavy chain protein in dexamethasone-treated skeletal muscle. Cell metabolism. 2007;6(5):376-85. doi: 10.1016/j.cmet.2007.09.009. PubMed PMID: 17983583.

70. Lagirand-Cantaloube J, Offner N, Csibi A, Leibovitch MP, Batonnet-Pichon S, Tintignac LA, et al. The initiation factor eIF3-f is a major target for atrogin1/MAFbx function in skeletal muscle atrophy. The EMBO journal. 2008;27(8):1266-76. doi: 10.1038/emboj.2008.52. PubMed PMID: 18354498; PubMed Central PMCID: PMC2367397.

71. Tintignac LA, Lagirand J, Batonnet S, Sirri V, Leibovitch MP, Leibovitch SA. Degradation of MyoD mediated by the SCF (MAFbx) ubiquitin ligase. The Journal of biological chemistry. 2005;280(4):2847-56. doi: 10.1074/jbc.M411346200. PubMed PMID: 15531760 .

72. Li HH, Kedar V, Zhang C, McDonough H, Arya R, Wang DZ, et al. Atrogin1/muscle atrophy F-box inhibits calcineurin-dependent cardiac hypertrophy by participating in an SCF ubiquitin ligase complex. The Journal of clinical investigation. 2004;114(8):1058- 
71. doi: 10.1172/JCI22220. PubMed PMID: 15489953; PubMed Central PMCID: PMC522252.

73. Cohen S, Zhai B, Gygi SP, Goldberg AL. Ubiquitylation by Trim32 causes coupled loss of desmin, Z-bands, and thin filaments in muscle atrophy. The Journal of cell biology. 2012;198(4):575-89. doi: 10.1083/jcb.201110067. PubMed PMID: 22908310; PubMed Central PMCID: PMCPMC3514026.

74. Paul PK, Gupta SK, Bhatnagar S, Panguluri SK, Darnay BG, Choi Y, et al. Targeted ablation of TRAF6 inhibits skeletal muscle wasting in mice. The Journal of cell biology. 2010;191(7):1395-411. doi: 10.1083/jcb.201006098. PubMed PMID: 21187332; PubMed Central PMCID: PMCPMC3010064.

75. Sun YS, Ye ZY, Qian ZY, Xu XD, Hu JF. Expression of TRAF6 and ubiquitin mRNA in skeletal muscle of gastric cancer patients. J Exp Clin Cancer Res. 2012;31:81. doi: 10.1186/1756-9966-31-81. PubMed PMID: 23013936; PubMed Central PMCID: PMCPMC3523060.

76. Isakson P, Lystad AH, Breen K, Koster G, Stenmark H, Simonsen A. TRAF6 mediates ubiquitination of KIF23/MKLP1 and is required for midbody ring degradation by selective autophagy. Autophagy. 2013;9(12):1955-64. PubMed PMID: 24128730.

77. Nazio F, Strappazzon F, Antonioli M, Bielli P, Cianfanelli V, Bordi M, et al. mTOR inhibits autophagy by controlling ULK1 ubiquitylation, self-association and function through AMBRA1 and TRAF6. Nature cell biology. 2013;15(4):406-16. doi: 10.1038/ncb2708. PubMed PMID: 23524951.

78. Neel BA, Lin Y, Pessin JE. Skeletal muscle autophagy: a new metabolic regulator. Trends in endocrinology and metabolism: TEM. 2013;24(12):635-43. doi: 10.1016/j.tem.2013.09.004. PubMed PMID: 24182456; PubMed Central PMCID: PMC3849822.

79. Mizushima N. Autophagy: process and function. Genes \& development. 2007;21(22):2861-73. doi: 10.1101/gad.1599207. PubMed PMID: 18006683.

80. Kaur J, Debnath J. Autophagy at the crossroads of catabolism and anabolism. Nat Rev Mol Cell Biol. 2015;16(8):461-72. doi: 10.1038/nrm4024. PubMed PMID: 26177004.

81. Axe EL, Walker SA, Manifava M, Chandra P, Roderick HL, Habermann A, et al. Autophagosome formation from membrane compartments enriched in phosphatidylinositol 3phosphate and dynamically connected to the endoplasmic reticulum. The Journal of cell biology. 2008;182(4):685-701. doi: 10.1083/jcb.200803137. PubMed PMID: 18725538; PubMed Central PMCID: PMCPMC2518708.

82. Ravikumar B, Moreau K, Jahreiss L, Puri C, Rubinsztein DC. Plasma membrane contributes to the formation of pre-autophagosomal structures. Nature cell biology. 2010;12(8):747-57. doi: 10.1038/ncb2078. PubMed PMID: 20639872; PubMed Central PMCID: PMCPMC2923063.

83. Hailey DW, Rambold AS, Satpute-Krishnan P, Mitra K, Sougrat R, Kim PK, et al. Mitochondria supply membranes for autophagosome biogenesis during starvation. Cell. 2010;141(4):656-67. doi: 10.1016/j.cell.2010.04.009. PubMed PMID: 20478256; PubMed Central PMCID: PMCPMC3059894.

84. Yamamoto H, Kakuta S, Watanabe TM, Kitamura A, Sekito T, Kondo-Kakuta C, et al. Atg9 vesicles are an important membrane source during early steps of autophagosome formation. The Journal of cell biology. 2012;198(2):219-33. doi: 10.1083/jcb.201202061. PubMed PMID: 22826123; PubMed Central PMCID: PMCPMC3410421.

85. Li WW, Li J, Bao JK. Microautophagy: lesser-known self-eating. Cell Mol Life Sci. 2012;69(7):1125-36. doi: 10.1007/s00018-011-0865-5. PubMed PMID: 22080117. 
86. Cuervo AM, Wong E. Chaperone-mediated autophagy: roles in disease and aging. Cell research. 2014;24(1):92-104. doi: 10.1038/cr.2013.153. PubMed PMID: 24281265; PubMed Central PMCID: PMCPMC3879702.

87. Grumati P, Coletto L, Schiavinato A, Castagnaro S, Bertaggia E, Sandri M, et al. Physical exercise stimulates autophagy in normal skeletal muscles but is detrimental for collagen VI-deficient muscles. Autophagy. 2011;7(12):1415-23. PubMed PMID: 22024752; PubMed Central PMCID: PMC3288016.

88. Jamart C, Benoit N, Raymackers JM, Kim HJ, Kim CK, Francaux M. Autophagyrelated and autophagy-regulatory genes are induced in human muscle after ultraendurance exercise. European journal of applied physiology. 2012;112(8):3173-7. doi: 10.1007/s00421011-2287-3. PubMed PMID: 22194006.

89. Jamart C, Francaux M, Millet GY, Deldicque L, Frere D, Feasson L. Modulation of autophagy and ubiquitin-proteasome pathways during ultra-endurance running. Journal of applied physiology. 2012;112(9):1529-37. doi: 10.1152/japplphysiol.00952.2011. PubMed PMID: 22345427.

90. Penna F, Costamagna D, Pin F, Camperi A, Fanzani A, Chiarpotto EM, et al. Autophagic degradation contributes to muscle wasting in cancer cachexia. The American journal of pathology. 2013;182(4):1367-78. doi: 10.1016/j.ajpath.2012.12.023. PubMed PMID: 23395093.

91. Johns N, Hatakeyama S, Stephens NA, Degen M, Degen S, Frieauff W, et al. Clinical classification of cancer cachexia: phenotypic correlates in human skeletal muscle. PloS one. 2014;9(1):e83618. doi: 10.1371/journal.pone.0083618. PubMed PMID: 24404136; PubMed Central PMCID: PMCPMC3880262.

92. Tardif N, Klaude M, Lundell L, Thorell A, Rooyackers O. Autophagic-lysosomal pathway is the main proteolytic system modified in the skeletal muscle of esophageal cancer patients. The American journal of clinical nutrition. 2013;98(6):1485-92. doi:

10.3945/ajcn.113.063859. PubMed PMID: 24108784.

93. Op den Kamp CM, Langen RC, Snepvangers FJ, de Theije CC, Schellekens JM, Laugs F, et al. Nuclear transcription factor kappa B activation and protein turnover adaptations in skeletal muscle of patients with progressive stages of lung cancer cachexia. The American journal of clinical nutrition. 2013;98(3):738-48. doi: 10.3945/ajen.113.058388. PubMed PMID: 23902785.

94. Aversa Z, Pin F, Lucia S, Penna F, Verzaro R, Fazi M, et al. Autophagy is induced in the skeletal muscle of cachectic cancer patients. Sci Rep. 2016;6:30340. doi: 10.1038/srep30340. PubMed PMID: 27459917; PubMed Central PMCID: PMCPMC4962093.

95. Masiero E, Agatea L, Mammucari C, Blaauw B, Loro E, Komatsu M, et al. Autophagy is required to maintain muscle mass. Cell metabolism. 2009;10(6):507-15. doi: 10.1016/j.cmet.2009.10.008. PubMed PMID: 19945408.

96. Raben N, Hill V, Shea L, Takikita S, Baum R, Mizushima N, et al. Suppression of autophagy in skeletal muscle uncovers the accumulation of ubiquitinated proteins and their potential role in muscle damage in Pompe disease. Human molecular genetics. 2008;17(24):3897-908. doi: 10.1093/hmg/ddn292. PubMed PMID: 18782848; PubMed Central PMCID: PMC2638578.

97. Martin JJ, de Barsy T, van Hoof F, Palladini G. Pompe's disease: an inborn lysosomal disorder with storage of glycogen. A study of brain and striated muscle. Acta neuropathologica. 1973;23(3):229-44. PubMed PMID: 4511788.

98. Nishino I, Fu J, Tanji K, Yamada T, Shimojo S, Koori T, et al. Primary LAMP-2 deficiency causes X-linked vacuolar cardiomyopathy and myopathy (Danon disease). Nature. 2000;406(6798):906-10. doi: 10.1038/35022604. PubMed PMID: 10972294. 
99. Risson V, Mazelin L, Roceri M, Sanchez H, Moncollin V, Corneloup C, et al. Muscle inactivation of mTOR causes metabolic and dystrophin defects leading to severe myopathy. The Journal of cell biology. 2009;187(6):859-74. doi: 10.1083/jcb.200903131. PubMed PMID: 20008564; PubMed Central PMCID: PMC2806319.

100. Chang NC, Nguyen M, Bourdon J, Risse PA, Martin J, Danialou G, et al. Bcl-2associated autophagy regulator Naf-1 required for maintenance of skeletal muscle. Human molecular genetics. 2012;21(10):2277-87. doi: 10.1093/hmg/dds048. PubMed PMID: 22343142 .

101. Bentzinger CF, Romanino K, Cloetta D, Lin S, Mascarenhas JB, Oliveri F, et al. Skeletal muscle-specific ablation of raptor, but not of rictor, causes metabolic changes and results in muscle dystrophy. Cell metabolism. 2008;8(5):411-24. doi:

10.1016/j.cmet.2008.10.002. PubMed PMID: 19046572.

102. White JP, Puppa MJ, Gao S, Sato S, Welle SL, Carson JA. Muscle mTORC1 suppression by IL-6 during cancer cachexia: a role for AMPK. American journal of physiology Endocrinology and metabolism. 2013;304(10):E1042-52. doi:

10.1152/ajpendo.00410.2012. PubMed PMID: 23531613; PubMed Central PMCID: PMC3651620.

103. Williams JP, Phillips BE, Smith K, Atherton PJ, Rankin D, Selby AL, et al. Effect of tumor burden and subsequent surgical resection on skeletal muscle mass and protein turnover in colorectal cancer patients. The American journal of clinical nutrition. 2012;96(5):1064-70. doi: 10.3945/ajcn.112.045708. PubMed PMID: 23034966.

104. Latres E, Amini AR, Amini AA, Griffiths J, Martin FJ, Wei Y, et al. Insulin-like growth factor-1 (IGF-1) inversely regulates atrophy-induced genes via the phosphatidylinositol 3-kinase/Akt/mammalian target of rapamycin (PI3K/Akt/mTOR) pathway. The Journal of biological chemistry. 2005;280(4):2737-44. doi: 10.1074/jbc.M407517200. PubMed PMID: 15550386.

105. Schiaffino S, Mammucari C. Regulation of skeletal muscle growth by the IGF1Akt/PKB pathway: insights from genetic models. Skelet Muscle. 2011;1(1):4. doi: 10.1186/2044-5040-1-4. PubMed PMID: 21798082; PubMed Central PMCID: PMCPMC3143906.

106. Inoki K, Li Y, Zhu T, Wu J, Guan KL. TSC2 is phosphorylated and inhibited by Akt and suppresses mTOR signalling. Nature cell biology. 2002;4(9):648-57. doi: 10.1038/ncb839. PubMed PMID: 12172553.

107. Tee AR, Manning BD, Roux PP, Cantley LC, Blenis J. Tuberous sclerosis complex gene products, Tuberin and Hamartin, control mTOR signaling by acting as a GTPaseactivating protein complex toward Rheb. Current biology : CB. 2003;13(15):1259-68. PubMed PMID: 12906785.

108. Sato T, Nakashima A, Guo L, Tamanoi F. Specific activation of mTORC1 by Rheb G-protein in vitro involves enhanced recruitment of its substrate protein. The Journal of biological chemistry. 2009;284(19):12783-91. doi: 10.1074/jbc.M809207200. PubMed PMID: 19299511; PubMed Central PMCID: PMC2676008.

109. Inoki K, Li Y, Xu T, Guan KL. Rheb GTPase is a direct target of TSC2 GAP activity and regulates mTOR signaling. Genes \& development. 2003;17(15):1829-34. doi: 10.1101/gad.1110003. PubMed PMID: 12869586; PubMed Central PMCID: PMC196227. 110. Sancak Y, Thoreen CC, Peterson TR, Lindquist RA, Kang SA, Spooner E, et al. PRAS40 is an insulin-regulated inhibitor of the mTORC1 protein kinase. Molecular cell. 2007;25(6):903-15. doi: 10.1016/j.molcel.2007.03.003. PubMed PMID: 17386266.

111. Hara K, Yonezawa K, Kozlowski MT, Sugimoto T, Andrabi K, Weng QP, et al. Regulation of eIF-4E BP1 phosphorylation by mTOR. The Journal of biological chemistry. 1997;272(42):26457-63. PubMed PMID: 9334222. 
112. Montagne J, Stewart MJ, Stocker H, Hafen E, Kozma SC, Thomas G. Drosophila S6 kinase: a regulator of cell size. Science. 1999;285(5436):2126-9. PubMed PMID: 10497130. 113. Proud CG. Signalling to translation: how signal transduction pathways control the protein synthetic machinery. The Biochemical journal. 2007;403(2):217-34. doi: 10.1042/BJ20070024. PubMed PMID: 17376031.

114. Ravikumar B, Vacher C, Berger Z, Davies JE, Luo S, Oroz LG, et al. Inhibition of mTOR induces autophagy and reduces toxicity of polyglutamine expansions in fly and mouse models of Huntington disease. Nature genetics. 2004;36(6):585-95. doi: 10.1038/ng1362. PubMed PMID: 15146184.

115. Ganley IG, Lam du H, Wang J, Ding X, Chen S, Jiang X. ULK1.ATG13.FIP200 complex mediates mTOR signaling and is essential for autophagy. The Journal of biological chemistry. 2009;284(18):12297-305. doi: 10.1074/jbc.M900573200. PubMed PMID: 19258318; PubMed Central PMCID: PMC2673298.

116. Sandri M, Sandri C, Gilbert A, Skurk C, Calabria E, Picard A, et al. Foxo transcription factors induce the atrophy-related ubiquitin ligase atrogin-1 and cause skeletal muscle atrophy. Cell. 2004;117(3):399-412. PubMed PMID: 15109499; PubMed Central PMCID: PMC3619734.

117. Zhao J, Brault JJ, Schild A, Cao P, Sandri M, Schiaffino S, et al. FoxO3 coordinately activates protein degradation by the autophagic/lysosomal and proteasomal pathways in atrophying muscle cells. Cell metabolism. 2007;6(6):472-83. doi:

10.1016/j.cmet.2007.11.004. PubMed PMID: 18054316.

118. Mammucari C, Milan G, Romanello V, Masiero E, Rudolf R, Del Piccolo P, et al. FoxO3 controls autophagy in skeletal muscle in vivo. Cell metabolism. 2007;6(6):458-71. doi: 10.1016/j.cmet.2007.11.001. PubMed PMID: 18054315.

119. Sacheck JM, Ohtsuka A, McLary SC, Goldberg AL. IGF-I stimulates muscle growth by suppressing protein breakdown and expression of atrophy-related ubiquitin ligases, atrogin-1 and MuRF1. American journal of physiology Endocrinology and metabolism. 2004;287(4):E591-601. doi: 10.1152/ajpendo.00073.2004. PubMed PMID: 15100091. 120. Reed SA, Sandesara PB, Senf SM, Judge AR. Inhibition of FoxO transcriptional activity prevents muscle fiber atrophy during cachexia and induces hypertrophy. FASEB journal : official publication of the Federation of American Societies for Experimental Biology. 2012;26(3):987-1000. doi: 10.1096/fj.11-189977. PubMed PMID: 22102632; PubMed Central PMCID: PMC3289501.

121. Stitt TN, Drujan D, Clarke BA, Panaro F, Timofeyva Y, Kline WO, et al. The IGF1/PI3K/Akt pathway prevents expression of muscle atrophy-induced ubiquitin ligases by inhibiting FOXO transcription factors. Molecular cell. 2004;14(3):395-403. PubMed PMID: 15125842.

122. Lee SW, Dai G, Hu Z, Wang X, Du J, Mitch WE. Regulation of muscle protein degradation: coordinated control of apoptotic and ubiquitin-proteasome systems by phosphatidylinositol 3 kinase. Journal of the American Society of Nephrology : JASN. 2004;15(6):1537-45. PubMed PMID: 15153564.

123. Kamei Y, Miura S, Suzuki M, Kai Y, Mizukami J, Taniguchi T, et al. Skeletal muscle FOXO1 (FKHR) transgenic mice have less skeletal muscle mass, down-regulated Type I (slow twitch/red muscle) fiber genes, and impaired glycemic control. The Journal of biological chemistry. 2004;279(39):41114-23. doi: 10.1074/jbc.M400674200. PubMed PMID: 15272020.

124. Southgate RJ, Neill B, Prelovsek O, El-Osta A, Kamei Y, Miura S, et al. FOXO1 regulates the expression of 4E-BP1 and inhibits mTOR signaling in mammalian skeletal muscle. The Journal of biological chemistry. 2007;282(29):21176-86. doi: 10.1074/jbc.M702039200. PubMed PMID: 17510058. 
125. Theologides A. Cancer cachexia. Cancer. 1979;43(5 Suppl):2004-12. PubMed PMID: 376104.

126. Fearon KC, Glass DJ, Guttridge DC. Cancer cachexia: mediators, signaling, and metabolic pathways. Cell metabolism. 2012;16(2):153-66. doi: 10.1016/j.cmet.2012.06.011. PubMed PMID: 22795476.

127. Tracey KJ, Wei H, Manogue KR, Fong Y, Hesse DG, Nguyen HT, et al. Cachectin/tumor necrosis factor induces cachexia, anemia, and inflammation. The Journal of experimental medicine. 1988;167(3):1211-27. PubMed PMID: 3351436; PubMed Central PMCID: PMC2188883.

128. Oliff A, Defeo-Jones D, Boyer M, Martinez D, Kiefer D, Vuocolo G, et al. Tumors secreting human TNF/cachectin induce cachexia in mice. Cell. 1987;50(4):555-63. PubMed PMID: 3607879.

129. Sishi BJ, Engelbrecht AM. Tumor necrosis factor alpha (TNF-alpha) inactivates the PI3-kinase/PKB pathway and induces atrophy and apoptosis in L6 myotubes. Cytokine. 2011;54(2):173-84. doi: 10.1016/j.cyto.2011.01.009. PubMed PMID: 21300557.

130. Moylan JS, Smith JD, Chambers MA, McLoughlin TJ, Reid MB. TNF induction of atrogin-1/MAFbx mRNA depends on Foxo4 expression but not AKT-Foxo1/3 signaling.

American journal of physiology Cell physiology. 2008;295(4):C986-93. doi:

10.1152/ajpcell.00041.2008. PubMed PMID: 18701653; PubMed Central PMCID: PMC2575831.

131. Jatoi A, Ritter HL, Dueck A, Nguyen PL, Nikcevich DA, Luyun RF, et al. A placebocontrolled, double-blind trial of infliximab for cancer-associated weight loss in elderly and/or poor performance non-small cell lung cancer patients (N01C9). Lung cancer.

2010;68(2):234-9. doi: 10.1016/j.lungcan.2009.06.020. PubMed PMID: 19665818.

132. Maltoni M, Fabbri L, Nanni O, Scarpi E, Pezzi L, Flamini E, et al. Serum levels of tumour necrosis factor alpha and other cytokines do not correlate with weight loss and anorexia in cancer patients. Supportive care in cancer : official journal of the Multinational Association of Supportive Care in Cancer. 1997;5(2):130-5. PubMed PMID: 9069613.

133. Carson JA, Baltgalvis KA. Interleukin 6 as a key regulator of muscle mass during cachexia. Exercise and sport sciences reviews. 2010;38(4):168-76. doi:

10.1097/JES.0b013e3181f44f11. PubMed PMID: 20871233; PubMed Central PMCID: PMC3065300.

134. Iwase S, Murakami T, Saito Y, Nakagawa K. Steep elevation of blood interleukin-6 (IL-6) associated only with late stages of cachexia in cancer patients. European cytokine network. 2004;15(4):312-6. PubMed PMID: 15627639.

135. Strassmann G, Fong M, Kenney JS, Jacob CO. Evidence for the involvement of interleukin 6 in experimental cancer cachexia. The Journal of clinical investigation. 1992;89(5):1681-4. doi: 10.1172/JCI1 15767. PubMed PMID: 1569207; PubMed Central PMCID: PMC443047.

136. Bonetto A, Aydogdu T, Jin X, Zhang Z, Zhan R, Puzis L, et al. JAK/STAT3 pathway inhibition blocks skeletal muscle wasting downstream of IL-6 and in experimental cancer cachexia. American journal of physiology Endocrinology and metabolism.

2012;303(3):E410-21. doi: 10.1152/ajpendo.00039.2012. PubMed PMID: 22669242;

PubMed Central PMCID: PMC3423125.

137. Baltgalvis KA, Berger FG, Pena MM, Davis JM, White JP, Carson JA. Muscle

wasting and interleukin-6-induced atrogin-I expression in the cachectic Apc ( Min/+ ) mouse.

Pflugers Archiv : European journal of physiology. 2009;457(5):989-1001. doi:

10.1007/s00424-008-0574-6. PubMed PMID: 18712412; PubMed Central PMCID:

PMC2867110. 
138. Baltgalvis KA, Berger FG, Pena MM, Davis JM, Muga SJ, Carson JA. Interleukin-6 and cachexia in ApcMin/+ mice. American journal of physiology Regulatory, integrative and comparative physiology. 2008;294(2):R393-401. doi: 10.1152/ajpregu.00716.2007. PubMed PMID: 18056981.

139. Keller C, Steensberg A, Pilegaard H, Osada T, Saltin B, Pedersen BK, et al.

Transcriptional activation of the IL-6 gene in human contracting skeletal muscle: influence of muscle glycogen content. FASEB journal : official publication of the Federation of American Societies for Experimental Biology. 2001;15(14):2748-50. doi: 10.1096/fj.01-0507fje. PubMed PMID: 11687509.

140. McPherron AC, Lawler AM, Lee SJ. Regulation of skeletal muscle mass in mice by a new TGF-beta superfamily member. Nature. 1997;387(6628):83-90. doi: 10.1038/387083a0. PubMed PMID: 9139826.

141. Kambadur R, Sharma M, Smith TP, Bass JJ. Mutations in myostatin (GDF8) in double-muscled Belgian Blue and Piedmontese cattle. Genome research. 1997;7(9):910-6. PubMed PMID: 9314496.

142. Grobet L, Martin LJ, Poncelet D, Pirottin D, Brouwers B, Riquet J, et al. A deletion in the bovine myostatin gene causes the double-muscled phenotype in cattle. Nature genetics. 1997;17(1):71-4. doi: 10.1038/ng0997-71. PubMed PMID: 9288100.

143. Schuelke M, Wagner KR, Stolz LE, Hubner C, Riebel T, Komen W, et al. Myostatin mutation associated with gross muscle hypertrophy in a child. The New England journal of medicine. 2004;350(26):2682-8. doi: 10.1056/NEJMoa040933. PubMed PMID: 15215484. 144. Zimmers TA, Davies MV, Koniaris LG, Haynes P, Esquela AF, Tomkinson KN, et al. Induction of cachexia in mice by systemically administered myostatin. Science. 2002;296(5572):1486-8. doi: 10.1126/science.1069525. PubMed PMID: 12029139.

145. Awano H, Takeshima Y, Okizuka Y, Saiki K, Yagi M, Matsuo M. Wide ranges of serum myostatin concentrations in Duchenne muscular dystrophy patients. Clinica chimica acta; international journal of clinical chemistry. 2008;391(1-2):115-7. doi:

10.1016/j.cca.2008.01.024. PubMed PMID: 18284920.

146. Bogdanovich S, Krag TO, Barton ER, Morris LD, Whittemore LA, Ahima RS, et al. Functional improvement of dystrophic muscle by myostatin blockade. Nature.

2002;420(6914):418-21. doi: 10.1038/nature01154. PubMed PMID: 12459784.

147. Zachwieja JJ, Smith SR, Sinha-Hikim I, Gonzalez-Cadavid N, Bhasin S. Plasma myostatin-immunoreactive protein is increased after prolonged bed rest with low-dose T3 administration. Journal of gravitational physiology : a journal of the International Society for Gravitational Physiology. 1999;6(2):11-5. PubMed PMID: 11543081.

148. Gonzalez-Cadavid NF, Taylor WE, Yarasheski K, Sinha-Hikim I, Ma K, Ezzat S, et al. Organization of the human myostatin gene and expression in healthy men and HIVinfected men with muscle wasting. Proceedings of the National Academy of Sciences of the United States of America. 1998;95(25):14938-43. PubMed PMID: 9843994; PubMed Central PMCID: PMC24554.

149. Aversa Z, Bonetto A, Penna F, Costelli P, Di Rienzo G, Lacitignola A, et al. Changes in myostatin signaling in non-weight-losing cancer patients. Annals of surgical oncology. 2012;19(4):1350-6. doi: 10.1245/s10434-011-1720-5. PubMed PMID: 21519918. 150. Lee SJ. Regulation of muscle mass by myostatin. Annual review of cell and developmental biology. 2004;20:61-86. doi: 10.1146/annurev.cellbio.20.012103.135836. PubMed PMID: 15473835.

151. McFarlane C, Langley B, Thomas M, Hennebry A, Plummer E, Nicholas G, et al. Proteolytic processing of myostatin is auto-regulated during myogenesis. Developmental biology. 2005;283(1):58-69. doi: 10.1016/j.ydbio.2005.03.039. PubMed PMID: 15975431. 
152. Lee SJ, McPherron AC. Regulation of myostatin activity and muscle growth. Proceedings of the National Academy of Sciences of the United States of America. 2001;98(16):9306-11. doi: 10.1073/pnas.151270098. PubMed PMID: 11459935; PubMed Central PMCID: PMC55416.

153. Huet C, Li ZF, Liu HZ, Black RA, Galliano MF, Engvall E. Skeletal muscle cell hypertrophy induced by inhibitors of metalloproteases; myostatin as a potential mediator. American journal of physiology Cell physiology. 2001;281(5):C1624-34. PubMed PMID: 11600426.

154. Thies RS, Chen T, Davies MV, Tomkinson KN, Pearson AA, Shakey QA, et al. GDF8 propeptide binds to GDF-8 and antagonizes biological activity by inhibiting GDF-8 receptor binding. Growth Factors. 2001;18(4):251-9. PubMed PMID: 11519824.

155. Bogdanovich S, Perkins KJ, Krag TO, Whittemore LA, Khurana TS. Myostatin propeptide-mediated amelioration of dystrophic pathophysiology. FASEB journal : official publication of the Federation of American Societies for Experimental Biology. 2005;19(6):543-9. doi: 10.1096/fj.04-2796com. PubMed PMID: 15791004.

156. Hamrick MW, Arounleut P, Kellum E, Cain M, Immel D, Liang LF. Recombinant myostatin (GDF-8) propeptide enhances the repair and regeneration of both muscle and bone in a model of deep penetrant musculoskeletal injury. J Trauma. 2010;69(3):579-83. doi: 10.1097/TA.0b013e3181c451f4. PubMed PMID: 20173658; PubMed Central PMCID: PMCPMC3738012.

157. Arounleut P, Bialek P, Liang LF, Upadhyay S, Fulzele S, Johnson M, et al. A myostatin inhibitor (propeptide-Fc) increases muscle mass and muscle fiber size in aged mice but does not increase bone density or bone strength. Exp Gerontol. 2013;48(9):898-904. doi: 10.1016/j.exger.2013.06.004. PubMed PMID: 23832079; PubMed Central PMCID: PMCPMC3930487.

158. Funkenstein B, Rebhan Y. Expression, purification, renaturation and activation of fish myostatin expressed in Escherichia coli: facilitation of refolding and activity inhibition by myostatin prodomain. Protein Expr Purif. 2007;54(1):54-65. doi: 10.1016/j.pep.2007.02.002. PubMed PMID: 17383894.

159. Jin HJ, Dunn MA, Borthakur D, Kim YS. Refolding and purification of unprocessed porcine myostatin expressed in Escherichia coli. Protein Expr Purif. 2004;35(1):1-10. doi: 10.1016/j.pep.2004.01.001. PubMed PMID: 15039059.

160. Wolfman NM, McPherron AC, Pappano WN, Davies MV, Song K, Tomkinson KN, et al. Activation of latent myostatin by the BMP-1/tolloid family of metalloproteinases. Proceedings of the National Academy of Sciences of the United States of America. 2003;100(26):15842-6. doi: 10.1073/pnas.2534946100. PubMed PMID: 14671324; PubMed Central PMCID: PMC307655.

161. Lee SJ. Genetic analysis of the role of proteolysis in the activation of latent myostatin. PloS one. 2008;3(2):e1628. doi: 10.1371/journal.pone.0001628. PubMed PMID: 18286185; PubMed Central PMCID: PMCPMC2237902.

162. Cash JN, Rejon CA, McPherron AC, Bernard DJ, Thompson TB. The structure of myostatin:follistatin 288: insights into receptor utilization and heparin binding. The EMBO journal. 2009;28(17):2662-76. doi: 10.1038/emboj.2009.205. PubMed PMID: 19644449; PubMed Central PMCID: PMCPMC2738701.

163. Rebbapragada A, Benchabane H, Wrana JL, Celeste AJ, Attisano L. Myostatin signals through a transforming growth factor beta-like signaling pathway to block adipogenesis. Molecular and cellular biology. 2003;23(20):7230-42. PubMed PMID: 14517293; PubMed Central PMCID: PMC230332.

164. Massague J, Blain SW, Lo RS. TGFbeta signaling in growth control, cancer, and heritable disorders. Cell. 2000;103(2):295-309. PubMed PMID: 11057902. 
165. Zhu X, Topouzis S, Liang LF, Stotish RL. Myostatin signaling through Smad2, Smad 3 and Smad4 is regulated by the inhibitory Smad7 by a negative feedback mechanism. Cytokine. 2004;26(6):262-72. doi: 10.1016/j.cyto.2004.03.007. PubMed PMID: 15183844. 166. Chen Y, Lebrun JJ, Vale W. Regulation of transforming growth factor beta- and activin-induced transcription by mammalian Mad proteins. Proceedings of the National Academy of Sciences of the United States of America. 1996;93(23):12992-7. PubMed PMID: 8917532; PubMed Central PMCID: PMCPMC24034.

167. Zhao J, Crowe DL, Castillo C, Wuenschell C, Chai Y, Warburton D. Smad7 is a TGF-beta-inducible attenuator of Smad2/3-mediated inhibition of embryonic lung morphogenesis. Mech Dev. 2000;93(1-2):71-81. PubMed PMID: 10781941.

168. Zhao J, Shi W, Chen H, Warburton D. Smad7 and Smad6 differentially modulate transforming growth factor beta -induced inhibition of embryonic lung morphogenesis. The Journal of biological chemistry. 2000;275(31):23992-7. doi: 10.1074/jbc.M002433200. PubMed PMID: 10801843.

169. Philip B, Lu Z, Gao Y. Regulation of GDF-8 signaling by the p38 MAPK. Cellular signalling. 2005;17(3):365-75. doi: 10.1016/j.cellsig.2004.08.003. PubMed PMID: 15567067.

170. Yang W, Chen Y, Zhang Y, Wang X, Yang N, Zhu D. Extracellular signal-regulated kinase 1/2 mitogen-activated protein kinase pathway is involved in myostatin-regulated differentiation repression. Cancer research. 2006;66(3):1320-6. doi: 10.1158/00085472.CAN-05-3060. PubMed PMID: 16452185.

171. McFarlane C, Plummer E, Thomas M, Hennebry A, Ashby M, Ling N, et al. Myostatin induces cachexia by activating the ubiquitin proteolytic system through an NFkappaB-independent, FoxO1-dependent mechanism. Journal of cellular physiology. 2006;209(2):501-14. doi: 10.1002/jcp.20757. PubMed PMID: 16883577.

172. Trendelenburg AU, Meyer A, Rohner D, Boyle J, Hatakeyama S, Glass DJ. Myostatin reduces Akt/TORC1/p70S6K signaling, inhibiting myoblast differentiation and myotube size. American journal of physiology Cell physiology. 2009;296(6):C1258-70. doi:

10.1152/ajpcell.00105.2009. PubMed PMID: 19357233.

173. Thomas M, Langley B, Berry C, Sharma M, Kirk S, Bass J, et al. Myostatin, a negative regulator of muscle growth, functions by inhibiting myoblast proliferation. The Journal of biological chemistry. 2000;275(51):40235-43. doi: 10.1074/jbc.M004356200. PubMed PMID: 10976104.

174. Joulia D, Bernardi H, Garandel V, Rabenoelina F, Vernus B, Cabello G. Mechanisms involved in the inhibition of myoblast proliferation and differentiation by myostatin. Experimental cell research. 2003;286(2):263-75. PubMed PMID: 12749855.

175. Rios R, Carneiro I, Arce VM, Devesa J. Myostatin regulates cell survival during $\mathrm{C} 2 \mathrm{C} 12$ myogenesis. Biochemical and biophysical research communications. 2001;280(2):561-6. doi: 10.1006/bbrc.2000.4159. PubMed PMID: 11162556.

176. Spiller MP, Kambadur R, Jeanplong F, Thomas M, Martyn JK, Bass JJ, et al. The myostatin gene is a downstream target gene of basic helix-loop-helix transcription factor MyoD. Molecular and cellular biology. 2002;22(20):7066-82. PubMed PMID: 12242286; PubMed Central PMCID: PMC139803.

177. Langley B, Thomas M, Bishop A, Sharma M, Gilmour S, Kambadur R. Myostatin inhibits myoblast differentiation by down-regulating MyoD expression. The Journal of biological chemistry. 2002;277(51):49831-40. doi: 10.1074/jbc.M204291200. PubMed PMID: 12244043.

178. McFarlane C, Hui GZ, Amanda WZ, Lau HY, Lokireddy S, Xiaojia G, et al. Human myostatin negatively regulates human myoblast growth and differentiation. American journal 
of physiology Cell physiology. 2011;301(1):C195-203. doi: 10.1152/ajpcell.00012.2011. PubMed PMID: 21508334; PubMed Central PMCID: PMC3129832.

179. Conboy IM, Conboy MJ, Wagers AJ, Girma ER, Weissman IL, Rando TA.

Rejuvenation of aged progenitor cells by exposure to a young systemic environment. Nature.

2005;433(7027):760-4. doi: 10.1038/nature03260. PubMed PMID: 15716955.

180. McCroskery S, Thomas M, Maxwell L, Sharma M, Kambadur R. Myostatin negatively regulates satellite cell activation and self-renewal. The Journal of cell biology. 2003;162(6):1135-47. doi: 10.1083/jcb.200207056. PubMed PMID: 12963705; PubMed Central PMCID: PMC2172861.

181. McCroskery S, Thomas M, Platt L, Hennebry A, Nishimura T, McLeay L, et al. Improved muscle healing through enhanced regeneration and reduced fibrosis in myostatinnull mice. Journal of cell science. 2005;118(Pt 15):3531-41. doi: 10.1242/jcs.02482. PubMed PMID: 16079293.

182. Wagner KR, Liu X, Chang X, Allen RE. Muscle regeneration in the prolonged absence of myostatin. Proceedings of the National Academy of Sciences of the United States of America. 2005;102(7):2519-24. doi: 10.1073/pnas.0408729102. PubMed PMID: 15699335; PubMed Central PMCID: PMCPMC548322.

183. Wang Q, McPherron AC. Myostatin inhibition induces muscle fibre hypertrophy prior to satellite cell activation. The Journal of physiology. 2012;590(9):2151-65. doi: 10.1113/jphysiol.2011.226001. PubMed PMID: 22393251; PubMed Central PMCID: PMCPMC3447157.

184. Sartori R, Milan G, Patron M, Mammucari C, Blaauw B, Abraham R, et al. Smad2 and 3 transcription factors control muscle mass in adulthood. American journal of physiology Cell physiology. 2009;296(6):C1248-57. doi: 10.1152/ajpcell.00104.2009. PubMed PMID: 19357234.

185. Lee SJ, Huynh TV, Lee YS, Sebald SM, Wilcox-Adelman SA, Iwamori N, et al. Role of satellite cells versus myofibers in muscle hypertrophy induced by inhibition of the myostatin/activin signaling pathway. Proceedings of the National Academy of Sciences of the United States of America. 2012;109(35):E2353-60. doi: 10.1073/pnas.1206410109. PubMed PMID: 22869749; PubMed Central PMCID: PMCPMC3435227.

186. Rommel C, Bodine SC, Clarke BA, Rossman R, Nunez L, Stitt TN, et al. Mediation of IGF-1-induced skeletal myotube hypertrophy by PI(3)K/Akt/mTOR and PI(3)K/Akt/GSK3 pathways. Nature cell biology. 2001;3(11):1009-13. doi: 10.1038/ncb1101-1009. PubMed PMID: 11715022.

187. Lipina C, Kendall H, McPherron AC, Taylor PM, Hundal HS. Mechanisms involved in the enhancement of mammalian target of rapamycin signalling and hypertrophy in skeletal muscle of myostatin-deficient mice. FEBS Lett. 2010;584(11):2403-8. doi:

10.1016/j.febslet.2010.04.039. PubMed PMID: 20412806; PubMed Central PMCID: PMCPMC2874630.

188. Morissette MR, Cook SA, Buranasombati C, Rosenberg MA, Rosenzweig A. Myostatin inhibits IGF-I-induced myotube hypertrophy through Akt. American journal of physiology Cell physiology. 2009;297(5):C1124-32. doi: 10.1152/ajpcell.00043.2009. PubMed PMID: 19759331; PubMed Central PMCID: PMCPMC2777401.

189. Rodriguez J, Vernus B, Toubiana M, Jublanc E, Tintignac L, Leibovitch S, et al. Myostatin inactivation increases myotube size through regulation of translational initiation machinery. J Cell Biochem. 2011;112(12):3531-42. doi: 10.1002/jcb.23280. PubMed PMID: 21769921.

190. Welle S, Bhatt K, Pinkert CA. Myofibrillar protein synthesis in myostatin-deficient mice. American journal of physiology Endocrinology and metabolism. 2006;290(3):E409-15. doi: 10.1152/ajpendo.00433.2005. PubMed PMID: 16219664. 
191. Welle S, Burgess K, Mehta S. Stimulation of skeletal muscle myofibrillar protein synthesis, p70 S6 kinase phosphorylation, and ribosomal protein S6 phosphorylation by inhibition of myostatin in mature mice. American journal of physiology Endocrinology and metabolism. 2009;296(3):E567-72. doi: 10.1152/ajpendo.90862.2008. PubMed PMID: 19141683; PubMed Central PMCID: PMCPMC2660146.

192. Amirouche A, Durieux AC, Banzet S, Koulmann N, Bonnefoy R, Mouret C, et al. Down-regulation of Akt/mammalian target of rapamycin signaling pathway in response to myostatin overexpression in skeletal muscle. Endocrinology. 2009;150(1):286-94. doi: 10.1210/en.2008-0959. PubMed PMID: 18801898.

193. Hitachi K, Nakatani M, Tsuchida K. Myostatin signaling regulates Akt activity via the regulation of miR-486 expression. The international journal of biochemistry \& cell biology. 2014;47:93-103. doi: 10.1016/j.biocel.2013.12.003. PubMed PMID: 24342526.

194. Sartori R, Schirwis E, Blaauw B, Bortolanza S, Zhao J, Enzo E, et al. BMP signaling controls muscle mass. Nature genetics. 2013;45(11):1309-18. doi: 10.1038/ng.2772. PubMed PMID: 24076600.

195. Allen DL, Unterman TG. Regulation of myostatin expression and myoblast differentiation by FoxO and SMAD transcription factors. American journal of physiology Cell physiology. 2007;292(1):C188-99. doi: 10.1152/ajpcell.00542.2005. PubMed PMID: 16885393.

196. Mendias CL, Kayupov E, Bradley JR, Brooks SV, Claflin DR. Decreased specific force and power production of muscle fibers from myostatin-deficient mice are associated with a suppression of protein degradation. Journal of applied physiology. 2011;111(1):18591. doi: 10.1152/japplphysiol.00126.2011. PubMed PMID: 21565991; PubMed Central PMCID: PMCPMC3137541.

197. LeBrasseur NK, Schelhorn TM, Bernardo BL, Cosgrove PG, Loria PM, Brown TA. Myostatin inhibition enhances the effects of exercise on performance and metabolic outcomes in aged mice. The journals of gerontology Series A, Biological sciences and medical sciences. 2009;64(9):940-8. doi: 10.1093/gerona/glp068. PubMed PMID: 19483181. 198. Brault JJ, Jespersen JG, Goldberg AL. Peroxisome proliferator-activated receptor gamma coactivator 1alpha or 1beta overexpression inhibits muscle protein degradation, induction of ubiquitin ligases, and disuse atrophy. The Journal of biological chemistry. 2010;285(25):19460-71. doi: 10.1074/jbc.M1 10.113092. PubMed PMID: 20404331; PubMed Central PMCID: PMCPMC2885225.

199. Ruas JL, White JP, Rao RR, Kleiner S, Brannan KT, Harrison BC, et al. A PGClalpha isoform induced by resistance training regulates skeletal muscle hypertrophy. Cell. 2012;151(6):1319-31. doi: 10.1016/j.cell.2012.10.050. PubMed PMID: 23217713; PubMed Central PMCID: PMCPMC3520615.

200. Liu CM, Yang Z, Liu CW, Wang R, Tien P, Dale R, et al. Myostatin antisense RNAmediated muscle growth in normal and cancer cachexia mice. Gene therapy. 2008;15(3):15560. doi: 10.1038/sj.gt.3303016. PubMed PMID: 18033313.

201. Benny Klimek ME, Aydogdu T, Link MJ, Pons M, Koniaris LG, Zimmers TA. Acute inhibition of myostatin-family proteins preserves skeletal muscle in mouse models of cancer cachexia. Biochemical and biophysical research communications. 2010;391(3):1548-54. doi: 10.1016/j.bbrc.2009.12.123. PubMed PMID: 20036643.

202. Murphy KT, Chee A, Gleeson BG, Naim T, Swiderski K, Koopman R, et al. Antibody-directed myostatin inhibition enhances muscle mass and function in tumor-bearing mice. American journal of physiology Regulatory, integrative and comparative physiology. 2011;301(3):R716-26. doi: 10.1152/ajpregu.00121.2011. PubMed PMID: 21677277.

203. Jameson GS, Hamm JT, Weiss GJ, Alemany C, Anthony S, Basche M, et al. A multicenter, phase I, dose-escalation study to assess the safety, tolerability, and 
pharmacokinetics of etirinotecan pegol in patients with refractory solid tumors. Clinical cancer research : an official journal of the American Association for Cancer Research. 2013;19(1):268-78. doi: 10.1158/1078-0432.CCR-12-1201. PubMed PMID: 23136196. 204. Hedayati M, Nozhat Z, Hannani M. Can the Serum Level of Myostatin be Considered as an Informative Factor for Cachexia Prevention in Patients with Medullary Thyroid Cancer? Asian Pac J Cancer Prev. 2016;17 Spec No.:119-23. PubMed PMID: 27165248. 205. Loumaye A, de Barsy M, Nachit M, Lause P, Frateur L, van Maanen A, et al. Role of Activin A and myostatin in human cancer cachexia. The Journal of clinical endocrinology and metabolism. 2015;100(5):2030-8. doi: 10.1210/jc.2014-4318. PubMed PMID: 25751105. 206. D'Orlando C, Marzetti E, Francois S, Lorenzi M, Conti V, di Stasio E, et al. Gastric cancer does not affect the expression of atrophy-related genes in human skeletal muscle. Muscle \& nerve. 2014;49(4):528-33. doi: 10.1002/mus.23945. PubMed PMID: 23835743. 207. Bonetto A, Penna F, Aversa Z, Mercantini P, Baccino FM, Costelli P, et al. Early changes of muscle insulin-like growth factor-1 and myostatin gene expression in gastric cancer patients. Muscle \& nerve. 2013;48(3):387-92. doi: 10.1002/mus.23798. PubMed PMID: 23861230.

208. Langley B, Thomas M, McFarlane C, Gilmour S, Sharma M, Kambadur R. Myostatin inhibits rhabdomyosarcoma cell proliferation through an $\mathrm{Rb}$-independent pathway. Oncogene. 2004;23(2):524-34. doi: 10.1038/sj.onc.1207144. PubMed PMID: 14724580. 209. Rossi S, Stoppani E, Puri PL, Fanzani A. Differentiation of human rhabdomyosarcoma RD cells is regulated by reciprocal, functional interactions between myostatin, p38 and extracellular regulated kinase signalling pathways. European journal of cancer. 2011;47(7):1095-105. doi: 10.1016/j.ejca.2010.12.010. PubMed PMID: 21273059. 210. Liu Y, Cheng H, Zhou Y, Zhu Y, Bian R, Chen Y, et al. Myostatin induces mitochondrial metabolic alteration and typical apoptosis in cancer cells. Cell death $\&$ disease. 2013;4:e494. doi: 10.1038/cddis.2013.31. PubMed PMID: 23412387; PubMed Central PMCID: PMC3734823.

211. Jemal A, Bray F, Center MM, Ferlay J, Ward E, Forman D. Global cancer statistics. CA Cancer J Clin. 2011;61(2):69-90. doi: 10.3322/caac.20107. PubMed PMID: 21296855. 212. Nyasavajjala SM, Phillips BE, Lund JN, Williams JP. Creatinine and myoglobin are poor predictors of anaerobic threshold in colorectal cancer and health. Journal of cachexia, sarcopenia and muscle. 2015;6(2):125-31. doi: 10.1002/jcsm.12020. PubMed PMID: 26136188; PubMed Central PMCID: PMCPMC4458078.

213. Renfro LA, Loupakis F, Adams RA, Seymour MT, Heinemann V, Schmoll HJ, et al. Body Mass Index Is Prognostic in Metastatic Colorectal Cancer: Pooled Analysis of Patients From First-Line Clinical Trials in the ARCAD Database. Journal of clinical oncology : official journal of the American Society of Clinical Oncology. 2016;34(2):144-50. doi: 10.1200/JCO.2015.61.6441. PubMed PMID: 26503203.

214. Bapuji SB, Sawatzky JA. Understanding weight loss in patients with colorectal cancer: a human response to illness. Oncol Nurs Forum. 2010;37(3):303-10. doi: 10.1188/10.ONF.303-310. PubMed PMID: 20439214.

215. Fearon ER, Vogelstein B. A genetic model for colorectal tumorigenesis. Cell. 1990;61(5):759-67. PubMed PMID: 2188735.

216. Morin PJ, Sparks AB, Korinek V, Barker N, Clevers H, Vogelstein B, et al. Activation of beta-catenin-Tcf signaling in colon cancer by mutations in beta-catenin or APC. Science. 1997;275(5307):1787-90. PubMed PMID: 9065402.

217. Bienz M, Clevers H. Linking colorectal cancer to Wnt signaling. Cell. 2000;103(2):311-20. PubMed PMID: 11057903. 
218. Cancer Genome Atlas N. Comprehensive molecular characterization of human colon and rectal cancer. Nature. 2012;487(7407):330-7. doi: 10.1038/nature11252. PubMed PMID: 22810696; PubMed Central PMCID: PMC3401966.

219. Scherer SJ, Avdievich E, Edelmann W. Functional consequences of DNA mismatch repair missense mutations in murine models and their impact on cancer predisposition. Biochemical Society transactions. 2005;33(Pt 4):689-93. doi: 10.1042/BST0330689. PubMed PMID: 16042575.

220. Fishel R, Lescoe MK, Rao MR, Copeland NG, Jenkins NA, Garber J, et al. The human mutator gene homolog MSH2 and its association with hereditary nonpolyposis colon cancer. Cell. 1993;75(5):1027-38. PubMed PMID: 8252616.

221. Kane MF, Loda M, Gaida GM, Lipman J, Mishra R, Goldman H, et al. Methylation of the hMLH1 promoter correlates with lack of expression of hMLH1 in sporadic colon tumors and mismatch repair-defective human tumor cell lines. Cancer research. 1997;57(5):808-11. PubMed PMID: 9041175.

222. Drost J, van Jaarsveld RH, Ponsioen B, Zimberlin C, van Boxtel R, Buijs A, et al. Sequential cancer mutations in cultured human intestinal stem cells. Nature. 2015;521(7550):43-7. doi: 10.1038/nature14415. PubMed PMID: 25924068. 223. Rajagopalan H, Nowak MA, Vogelstein B, Lengauer C. The significance of unstable chromosomes in colorectal cancer. Nat Rev Cancer. 2003;3(9):695-701. doi: 10.1038/nrc1165. PubMed PMID: 12951588.

224. Thibodeau SN, Bren G, Schaid D. Microsatellite instability in cancer of the proximal colon. Science. 1993;260(5109):816-9. PubMed PMID: 8484122.

225. Blake C, Tsao JL, Wu A, Shibata D. Stepwise deletions of polyA sequences in mismatch repair-deficient colorectal cancers. The American journal of pathology. 2001;158(5):1867-70. doi: 10.1016/S0002-9440(10)64143-0. PubMed PMID: 11337385; PubMed Central PMCID: PMCPMC1891934.

226. Barker N, van de Wetering M, Clevers H. The intestinal stem cell. Genes \& development. 2008;22(14):1856-64. doi: 10.1101/gad.1674008. PubMed PMID: 18628392; PubMed Central PMCID: PMCPMC2735277.

227. Kim YS, Ho SB. Intestinal goblet cells and mucins in health and disease: recent insights and progress. Curr Gastroenterol Rep. 2010;12(5):319-30. doi: 10.1007/s11894-0100131-2. PubMed PMID: 20703838; PubMed Central PMCID: PMCPMC2933006.

228. Gallo RL, Hooper LV. Epithelial antimicrobial defence of the skin and intestine. Nat Rev Immunol. 2012;12(7):503-16. doi: 10.1038/nri3228. PubMed PMID: 22728527; PubMed Central PMCID: PMCPMC3563335.

229. Furness JB, Rivera LR, Cho HJ, Bravo DM, Callaghan B. The gut as a sensory organ. Nat Rev Gastroenterol Hepatol. 2013;10(12):729-40. doi: 10.1038/nrgastro.2013.180. PubMed PMID: 24061204.

230. Watson AJ, Hughes KR. TNF-alpha-induced intestinal epithelial cell shedding: implications for intestinal barrier function. Ann N Y Acad Sci. 2012;1258:1-8. doi: 10.1111/j.1749-6632.2012.06523.x. PubMed PMID: 22731709.

231. Leblond CP, Walker BE. Renewal of cell populations. Physiological reviews. 1956;36(2):255-76. PubMed PMID: 13322651.

232. Barker N. Adult intestinal stem cells: critical drivers of epithelial homeostasis and regeneration. Nat Rev Mol Cell Biol. 2014;15(1):19-33. doi: 10.1038/nrm3721. PubMed PMID: 24326621.

233. Winton DJ, Ponder BA. Stem-cell organization in mouse small intestine. Proc Biol Sci. 1990;241(1300):13-8. doi: 10.1098/rspb.1990.0059. PubMed PMID: 1978330.

234. Heath JP. Epithelial cell migration in the intestine. Cell Biol Int. 1996;20(2):139-46. doi: 10.1006/cbir.1996.0018. PubMed PMID: 8935158. 
235. Yatabe Y, Tavare S, Shibata D. Investigating stem cells in human colon by using methylation patterns. Proceedings of the National Academy of Sciences of the United States of America. 2001;98(19):10839-44. doi: 10.1073/pnas.191225998. PubMed PMID: 11517339; PubMed Central PMCID: PMCPMC58561.

236. Ireland H, Houghton C, Howard L, Winton DJ. Cellular inheritance of a Cre-activated reporter gene to determine Paneth cell longevity in the murine small intestine. Developmental dynamics : an official publication of the American Association of Anatomists.

2005;233(4):1332-6. doi: 10.1002/dvdy.20446. PubMed PMID: 15937933.

237. Barker N, van Es JH, Kuipers J, Kujala P, van den Born M, Cozijnsen M, et al. Identification of stem cells in small intestine and colon by marker gene Lgr5. Nature. 2007;449(7165):1003-7. doi: 10.1038/nature06196. PubMed PMID: 17934449.

238. Potten CS, Kovacs L, Hamilton E. Continuous labelling studies on mouse skin and intestine. Cell Tissue Kinet. 1974;7(3):271-83. PubMed PMID: 4837676.

239. Potten CS. Extreme sensitivity of some intestinal crypt cells to X and gamma irradiation. Nature. 1977;269(5628):518-21. PubMed PMID: 909602.

240. Potten CS, Owen G, Booth D. Intestinal stem cells protect their genome by selective segregation of template DNA strands. Journal of cell science. 2002;115(Pt 11):2381-8.

PubMed PMID: 12006622.

241. Sangiorgi E, Capecchi MR. Bmil is expressed in vivo in intestinal stem cells. Nature genetics. 2008;40(7):915-20. doi: 10.1038/ng.165. PubMed PMID: 18536716; PubMed Central PMCID: PMCPMC2906135.

242. Yan KS, Chia LA, Li X, Ootani A, Su J, Lee JY, et al. The intestinal stem cell markers Bmil and Lgr5 identify two functionally distinct populations. Proceedings of the National Academy of Sciences of the United States of America. 2012;109(2):466-71. doi: 10.1073/pnas.1118857109. PubMed PMID: 22190486; PubMed Central PMCID: PMCPMC3258636.

243. Buczacki SJ, Zecchini HI, Nicholson AM, Russell R, Vermeulen L, Kemp R, et al. Intestinal label-retaining cells are secretory precursors expressing Lgr5. Nature. 2013;495(7439):65-9. doi: 10.1038/nature11965. PubMed PMID: 23446353.

244. Barker N, Ridgway RA, van Es JH, van de Wetering M, Begthel H, van den Born M, et al. Crypt stem cells as the cells-of-origin of intestinal cancer. Nature. 2009;457(7229):60811. doi: 10.1038/nature07602. PubMed PMID: 19092804.

245. Powell AE, Vlacich G, Zhao ZY, McKinley ET, Washington MK, Manning HC, et al. Inducible loss of one Apc allele in Lrig1-expressing progenitor cells results in multiple distal colonic tumors with features of familial adenomatous polyposis. Am J Physiol Gastrointest Liver Physiol. 2014;307(1):G16-23. doi: 10.1152/ajpgi.00358.2013. PubMed PMID: 24833705; PubMed Central PMCID: PMCPMC4080164.

246. Powell AE, Wang Y, Li Y, Poulin EJ, Means AL, Washington MK, et al. The panErbB negative regulator Lrig1 is an intestinal stem cell marker that functions as a tumor suppressor. Cell. 2012;149(1):146-58. doi: 10.1016/j.cell.2012.02.042. PubMed PMID: 22464327; PubMed Central PMCID: PMCPMC3563328.

247. Zhu L, Gibson P, Currle DS, Tong Y, Richardson RJ, Bayazitov IT, et al. Prominin 1 marks intestinal stem cells that are susceptible to neoplastic transformation. Nature. 2009;457(7229):603-7. doi: 10.1038/nature07589. PubMed PMID: 19092805; PubMed Central PMCID: PMCPMC2633030.

248. Preston SL, Wong WM, Chan AO, Poulsom R, Jeffery R, Goodlad RA, et al. Bottomup histogenesis of colorectal adenomas: origin in the monocryptal adenoma and initial expansion by crypt fission. Cancer research. 2003;63(13):3819-25. PubMed PMID: 12839979. 
249. Shih IM, Wang TL, Traverso G, Romans K, Hamilton SR, Ben-Sasson S, et al. Topdown morphogenesis of colorectal tumors. Proceedings of the National Academy of Sciences of the United States of America. 2001;98(5):2640-5. doi: 10.1073/pnas.051629398. PubMed PMID: 11226292; PubMed Central PMCID: PMCPMC30191.

250. Shih IM, Zhou W, Goodman SN, Lengauer C, Kinzler KW, Vogelstein B. Evidence that genetic instability occurs at an early stage of colorectal tumorigenesis. Cancer research. 2001;61(3):818-22. PubMed PMID: 11221861.

251. Schwitalla S, Fingerle AA, Cammareri P, Nebelsiek T, Goktuna SI, Ziegler PK, et al. Intestinal tumorigenesis initiated by dedifferentiation and acquisition of stem-cell-like properties. Cell. 2013;152(1-2):25-38. doi: 10.1016/j.cell.2012.12.012. PubMed PMID: 23273993.

252. Moser AR, Luongo C, Gould KA, McNeley MK, Shoemaker AR, Dove WF. ApcMin: a mouse model for intestinal and mammary tumorigenesis. European journal of cancer. 1995;31A(7-8):1061-4. PubMed PMID: 7576992.

253. Mehl KA, Davis JM, Berger FG, Carson JA. Myofiber degeneration/regeneration is induced in the cachectic ApcMin/+ mouse. Journal of applied physiology. 2005;99(6):237987. doi: 10.1152/japplphysiol.00778.2005. PubMed PMID: 16288100.

254. Moser AR, Mattes EM, Dove WF, Lindstrom MJ, Haag JD, Gould MN. ApcMin, a mutation in the murine Apc gene, predisposes to mammary carcinomas and focal alveolar hyperplasias. Proceedings of the National Academy of Sciences of the United States of America. 1993;90(19):8977-81. PubMed PMID: 8415640; PubMed Central PMCID: PMC47484.

255. Moser AR, Pitot HC, Dove WF. A dominant mutation that predisposes to multiple intestinal neoplasia in the mouse. Science. 1990;247(4940):322-4. PubMed PMID: 2296722. 256. Su LK, Kinzler KW, Vogelstein B, Preisinger AC, Moser AR, Luongo C, et al. Multiple intestinal neoplasia caused by a mutation in the murine homolog of the APC gene. Science. 1992;256(5057):668-70. PubMed PMID: 1350108.

257. Haigis KM, Dove WF. A Robertsonian translocation suppresses a somatic recombination pathway to loss of heterozygosity. Nature genetics. 2003;33(1):33-9. doi: 10.1038/ng1055. PubMed PMID: 12447373.

258. Fearnhead NS, Britton MP, Bodmer WF. The ABC of APC. Human molecular genetics. 2001;10(7):721-33. PubMed PMID: 11257105.

259. Groden J, Thliveris A, Samowitz W, Carlson M, Gelbert L, Albertsen H, et al. Identification and characterization of the familial adenomatous polyposis coli gene. Cell. 1991;66(3):589-600. PubMed PMID: 1651174.

260. Lieffers JR, Mourtzakis M, Hall KD, McCargar LJ, Prado CM, Baracos VE. A viscerally driven cachexia syndrome in patients with advanced colorectal cancer: contributions of organ and tumor mass to whole-body energy demands. The American journal of clinical nutrition. 2009;89(4):1173-9. doi: 10.3945/ajen.2008.27273. PubMed PMID: 19244378; PubMed Central PMCID: PMC2667460.

261. Bennani-Baiti N, Walsh D. Animal models of the cancer anorexia-cachexia syndrome. Supportive care in cancer : official journal of the Multinational Association of Supportive Care in Cancer. 2011;19(9):1451-63. doi: 10.1007/s00520-010-0972-0. PubMed PMID: 20714754.

262. Moser AR, Dove WF, Roth KA, Gordon JI. The Min (multiple intestinal neoplasia) mutation: its effect on gut epithelial cell differentiation and interaction with a modifier system. The Journal of cell biology. 1992;116(6):1517-26. PubMed PMID: 1541640; PubMed Central PMCID: PMCPMC2289373. 
263. Dietrich WF, Lander ES, Smith JS, Moser AR, Gould KA, Luongo C, et al. Genetic identification of Mom-1, a major modifier locus affecting Min-induced intestinal neoplasia in the mouse. Cell. 1993;75(4):631-9. PubMed PMID: 8242739.

264. Gould KA, Dietrich WF, Borenstein N, Lander ES, Dove WF. Mom1 is a semidominant modifier of intestinal adenoma size and multiplicity in Min/+ mice. Genetics. 1996;144(4):1769-76. PubMed PMID: 8978062; PubMed Central PMCID:

PMCPMC1207726.

265. McCart AE, Vickaryous NK, Silver A. Apc mice: models, modifiers and mutants. Pathol Res Pract. 2008;204(7):479-90. doi: 10.1016/j.prp.2008.03.004. PubMed PMID: 18538487.

266. Silverman KA, Koratkar R, Siracusa LD, Buchberg AM. Identification of the modifier of Min 2 (Mom2) locus, a new mutation that influences Apc-induced intestinal neoplasia. Genome research. 2002;12(1):88-97. doi: 10.1101/gr.206002. PubMed PMID: 11779834; PubMed Central PMCID: PMCPMC155250.

267. Tanaka T, Kohno H, Suzuki R, Yamada Y, Sugie S, Mori H. A novel inflammationrelated mouse colon carcinogenesis model induced by azoxymethane and dextran sodium sulfate. Cancer Sci. 2003;94(11):965-73. PubMed PMID: 14611673.

268. Rosenberg DW, Giardina C, Tanaka T. Mouse models for the study of colon carcinogenesis. Carcinogenesis. 2009;30(2):183-96. doi: 10.1093/carcin/bgn267. PubMed PMID: 19037092; PubMed Central PMCID: PMCPMC2639048.

269. De Robertis M, Massi E, Poeta ML, Carotti S, Morini S, Cecchetelli L, et al. The AOM/DSS murine model for the study of colon carcinogenesis: From pathways to diagnosis and therapy studies. J Carcinog. 2011;10:9. doi: 10.4103/1477-3163.78279. PubMed PMID: 21483655 ; PubMed Central PMCID: PMCPMC3072657.

270. Delker DA, McKnight SJ, 3rd, Rosenberg DW. The role of alcohol dehydrogenase in the metabolism of the colon carcinogen methylazoxymethanol. Toxicol Sci. 1998;45(1):6671. doi: 10.1006/toxs.1998.2499. PubMed PMID: 9848112.

271. Sohn OS, Fiala ES, Requeijo SP, Weisburger JH, Gonzalez FJ. Differential effects of CYP2E1 status on the metabolic activation of the colon carcinogens azoxymethane and methylazoxymethanol. Cancer research. 2001;61(23):8435-40. PubMed PMID: 11731424.

272. Okayasu I, Hatakeyama S, Yamada M, Ohkusa T, Inagaki Y, Nakaya R. A novel method in the induction of reliable experimental acute and chronic ulcerative colitis in mice. Gastroenterology. 1990;98(3):694-702. PubMed PMID: 1688816.

273. Thaker AI, Shaker A, Rao MS, Ciorba MA. Modeling colitis-associated cancer with azoxymethane (AOM) and dextran sulfate sodium (DSS). J Vis Exp. 2012;(67). doi:

10.3791/4100. PubMed PMID: 22990604; PubMed Central PMCID: PMCPMC3490277.

274. Maltzman T, Whittington J, Driggers L, Stephens J, Ahnen D. AOM-induced mouse colon tumors do not express full-length APC protein. Carcinogenesis. 1997;18(12):2435-9.

PubMed PMID: 9450492.

275. Takahashi M, Nakatsugi S, Sugimura T, Wakabayashi K. Frequent mutations of the beta-catenin gene in mouse colon tumors induced by azoxymethane. Carcinogenesis.

2000;21(6):1117-20. PubMed PMID: 10836998.

276. Vivona AA, Shpitz B, Medline A, Bruce WR, Hay K, Ward MA, et al. K-ras mutations in aberrant crypt foci, adenomas and adenocarcinomas during azoxymethaneinduced colon carcinogenesis. Carcinogenesis. 1993;14(9):1777-81. PubMed PMID: 8403199.

277. Yasui Y, Tanaka T. Protein expression analysis of inflammation-related colon carcinogenesis. J Carcinog. 2009;8:10. doi: 10.4103/1477-3163.51851. PubMed PMID: 19491504; PubMed Central PMCID: PMCPMC2699605. 
278. Suzuki R, Miyamoto S, Yasui Y, Sugie S, Tanaka T. Global gene expression analysis of the mouse colonic mucosa treated with azoxymethane and dextran sodium sulfate. BMC cancer. 2007;7:84. doi: 10.1186/1471-2407-7-84. PubMed PMID: 17506908; PubMed Central PMCID: PMCPMC1890554.

279. Baltgalvis KA, Berger FG, Pena MM, Mark Davis J, White JP, Carson JA. Activity level, apoptosis, and development of cachexia in Apc(Min/+) mice. Journal of applied physiology. 2010;109(4):1155-61. doi: 10.1152/japplphysiol.00442.2010. PubMed PMID: 20651218 ; PubMed Central PMCID: PMC2963323.

280. Zhang C, McFarlane C, Lokireddy S, Masuda S, Ge X, Gluckman PD, et al. Inhibition of myostatin protects against diet-induced obesity by enhancing fatty acid oxidation and promoting a brown adipose phenotype in mice. Diabetologia. 2012;55(1):18393. doi: 10.1007/s00125-011-2304-4. PubMed PMID: 21927895.

281. Li ZF, Shelton GD, Engvall E. Elimination of myostatin does not combat muscular dystrophy in dy mice but increases postnatal lethality. American Journal of Pathology. 2006. 282. Rachagani S, Cheng Y, Reecy JM. Myostatin genotype regulates muscle-specific miRNA expression in mouse pectoralis muscle. BMC Res Notes. 2010;3:297. doi: 10.1186/1756-0500-3-297. PubMed PMID: 21070642; PubMed Central PMCID: PMCPMC2992544.

283. Sato T, Stange DE, Ferrante M, Vries RG, Van Es JH, Van den Brink S, et al. Longterm expansion of epithelial organoids from human colon, adenoma, adenocarcinoma, and Barrett's epithelium. Gastroenterology. 2011;141(5):1762-72. doi:

10.1053/j.gastro.2011.07.050. PubMed PMID: 21889923.

284. Cohen S, Nathan JA, Goldberg AL. Muscle wasting in disease: molecular mechanisms and promising therapies. Nat Rev Drug Discov. 2015;14(1):58-74. doi: 10.1038/nrd4467. PubMed PMID: 25549588.

285. Zhang L, Pan J, Dong Y, Tweardy DJ, Dong Y, Garibotto G, et al. Stat3 activation links a C/EBPdelta to myostatin pathway to stimulate loss of muscle mass. Cell metabolism. 2013;18(3):368-79. doi: 10.1016/j.cmet.2013.07.012. PubMed PMID: 24011072; PubMed Central PMCID: PMCPMC3794464.

286. Zhang L, Rajan V, Lin E, Hu Z, Han HQ, Zhou X, et al. Pharmacological inhibition of myostatin suppresses systemic inflammation and muscle atrophy in mice with chronic kidney disease. FASEB journal : official publication of the Federation of American Societies for Experimental Biology. 2011;25(5):1653-63. doi: 10.1096/fj.10-176917. PubMed PMID: 21282204; PubMed Central PMCID: PMCPMC3079306.

287. Wilkes JJ, Lloyd DJ, Gekakis N. Loss-of-function mutation in myostatin reduces tumor necrosis factor alpha production and protects liver against obesity-induced insulin resistance. Diabetes. 2009;58(5):1133-43. doi: 10.2337/db08-0245. PubMed PMID: 19208906; PubMed Central PMCID: PMCPMC2671051.

288. Yekkala K, Baudino TA. Inhibition of intestinal polyposis with reduced angiogenesis in ApcMin/+ mice due to decreases in c-Myc expression. Mol Cancer Res. 2007;5(12):1296303. doi: 10.1158/1541-7786.MCR-07-0232. PubMed PMID: 18171987.

289. Lane SW, Sykes SM, Al-Shahrour F, Shterental S, Paktinat M, Lo Celso C, et al. The Apc(min) mouse has altered hematopoietic stem cell function and provides a model for MPD/MDS. Blood. 2010;115(17):3489-97. doi: 10.1182/blood-2009-11-251728. PubMed PMID: 20197553; PubMed Central PMCID: PMCPMC2867262.

290. Brandt C, Nielsen AR, Fischer CP, Hansen J, Pedersen BK, Plomgaard P. Plasma and muscle myostatin in relation to type 2 diabetes. PloS one. 2012;7(5):e37236. doi:

10.1371/journal.pone.0037236. PubMed PMID: 22615949; PubMed Central PMCID: PMC3353926. 
291. Reardon KA, Davis J, Kapsa RM, Choong P, Byrne E. Myostatin, insulin-like growth factor-1, and leukemia inhibitory factor mRNAs are upregulated in chronic human disuse muscle atrophy. Muscle \& nerve. 2001;24(7):893-9. PubMed PMID: 11410916.

292. Gruson D, Ahn SA, Ketelslegers JM, Rousseau MF. Increased plasma myostatin in heart failure. European journal of heart failure. 2011;13(7):734-6. doi: 10.1093/eurjhf/hfr024. PubMed PMID: 21467027.

293. Sharma M, Kambadur R, Matthews KG, Somers WG, Devlin GP, Conaglen JV, et al. Myostatin, a transforming growth factor-beta superfamily member, is expressed in heart muscle and is upregulated in cardiomyocytes after infarct. Journal of cellular physiology. 1999;180(1):1-9. doi: 10.1002/(SICI)1097-4652(199907)180:1<1::AID-JCP1>3.0.CO;2-V. PubMed PMID: 10362012.

294. Aulino P, Berardi E, Cardillo VM, Rizzuto E, Perniconi B, Ramina C, et al. Molecular, cellular and physiological characterization of the cancer cachexia-inducing C26 colon carcinoma in mouse. BMC cancer. 2010;10:363. doi: 10.1186/1471-2407-10-363. PubMed PMID: 20615237; PubMed Central PMCID: PMC2912868.

295. Fearon KC, Hansell DT, Preston T, Plumb JA, Davies J, Shapiro D, et al. Influence of whole body protein turnover rate on resting energy expenditure in patients with cancer. Cancer research. 1988;48(9):2590-5. PubMed PMID: 3356019.

296. Mehl KA, Davis JM, Clements JM, Berger FG, Pena MM, Carson JA. Decreased intestinal polyp multiplicity is related to exercise mode and gender in ApcMin/+ mice. Journal of applied physiology. 2005;98(6):2219-25. doi: 10.1152/japplphysiol.00975.2004. PubMed PMID: 15894538.

297. White JP, Baynes JW, Welle SL, Kostek MC, Matesic LE, Sato S, et al. The regulation of skeletal muscle protein turnover during the progression of cancer cachexia in the Apc(Min/+) mouse. PloS one. 2011;6(9):e24650. doi: 10.1371/journal.pone.0024650. PubMed PMID: 21949739; PubMed Central PMCID: PMCPMC3176277.

298. Zhang L, Tang H, Kou Y, Li R, Zheng Y, Wang Q, et al. MG132-mediated inhibition of the ubiquitin-proteasome pathway ameliorates cancer cachexia. J Cancer Res Clin Oncol. 2013;139(7):1105-15. doi: 10.1007/s00432-013-1412-6. PubMed PMID: 23535871.

299. Baltgalvis KA, Berger FG, Pena MM, Davis JM, Carson JA. Effect of exercise on biological pathways in ApcMin/+ mouse intestinal polyps. Journal of applied physiology. 2008;104(4):1137-43. doi: 10.1152/japplphysiol.00955.2007. PubMed PMID: 18239078. 300. De Simone V, Franze E, Ronchetti G, Colantoni A, Fantini MC, Di Fusco D, et al. Th17-type cytokines, IL-6 and TNF-alpha synergistically activate STAT3 and NF-kB to promote colorectal cancer cell growth. Oncogene. 2015;34(27):3493-503. doi: 10.1038/onc.2014.286. PubMed PMID: 25174402; PubMed Central PMCID: PMCPMC4493653.

301. Han HQ, Zhou X, Mitch WE, Goldberg AL. Myostatin/activin pathway antagonism: molecular basis and therapeutic potential. The international journal of biochemistry \& cell biology. 2013;45(10):2333-47. doi: 10.1016/j.biocel.2013.05.019. PubMed PMID: 23721881. 302. Szlama G, Trexler M, Buday L, Patthy L. K153R polymorphism in myostatin gene increases the rate of promyostatin activation by furin. FEBS Lett. 2015;589(3):295-301. doi: 10.1016/j.febslet.2014.12.011. PubMed PMID: 25543063.

303. Hill JJ, Davies MV, Pearson AA, Wang JH, Hewick RM, Wolfman NM, et al. The myostatin propeptide and the follistatin-related gene are inhibitory binding proteins of myostatin in normal serum. The Journal of biological chemistry. 2002;277(43):40735-41. doi: 10.1074/jbc.M206379200. PubMed PMID: 12194980.

304. Kondas K, Szlama G, Trexler M, Patthy L. Both WFIKKN1 and WFIKKN2 have high affinity for growth and differentiation factors 8 and 11. The Journal of biological 
chemistry. 2008;283(35):23677-84. doi: 10.1074/jbc.M803025200. PubMed PMID: 18596030; PubMed Central PMCID: PMCPMC3259755.

305. Nakashima M, Toyono T, Akamine A, Joyner A. Expression of growth/differentiation factor 11, a new member of the BMP/TGFbeta superfamily during mouse embryogenesis. Mech Dev. 1999;80(2):185-9. PubMed PMID: 10072786.

306. Rodgers BD, Eldridge JA. Reduced Circulating GDF11 Is Unlikely Responsible for Age-Dependent Changes in Mouse Heart, Muscle, and Brain. Endocrinology. 2015;156(11):3885-8. doi: 10.1210/en.2015-1628. PubMed PMID: 26372181.

307. Schafer MJ, Atkinson EJ, Vanderboom PM, Kotajarvi B, White TA, Moore MM, et al. Quantification of GDF11 and Myostatin in Human Aging and Cardiovascular Disease. Cell metabolism. 2016;23(6):1207-15. doi: 10.1016/j.cmet.2016.05.023. PubMed PMID: 27304512; PubMed Central PMCID: PMCPMC4913514.

308. Kontermann RE. Strategies for extended serum half-life of protein therapeutics. Curr Opin Biotechnol. 2011;22(6):868-76. doi: 10.1016/j.copbio.2011.06.012. PubMed PMID: 21862310.

309. Roopenian DC, Akilesh S. FcRn: the neonatal Fc receptor comes of age. Nat Rev Immunol. 2007;7(9):715-25. doi: 10.1038/nri2155. PubMed PMID: 17703228.

310. Sako D, Grinberg AV, Liu J, Davies MV, Castonguay R, Maniatis S, et al. Characterization of the ligand binding functionality of the extracellular domain of activin receptor type IIb. The Journal of biological chemistry. 2010;285(27):21037-48. doi: 10.1074/jbc.M110.114959. PubMed PMID: 20385559; PubMed Central PMCID: PMC2898293.

311. Toledo M, Busquets S, Penna F, Zhou X, Marmonti E, Betancourt A, et al. Complete reversal of muscle wasting in experimental cancer cachexia: Additive effects of activin type II receptor inhibition and beta-2 agonist. International journal of cancer Journal international du cancer. 2016;138(8):2021-9. doi: 10.1002/ijc.29930. PubMed PMID: 26595367.

312. Gallot YS, Durieux AC, Castells J, Desgeorges MM, Vernus B, Plantureux L, et al. Myostatin gene inactivation prevents skeletal muscle wasting in cancer. Cancer research. 2014;74(24):7344-56. doi: 10.1158/0008-5472.CAN-14-0057. PubMed PMID: 25336187.

313. Neufert C, Becker C, Neurath MF. An inducible mouse model of colon carcinogenesis for the analysis of sporadic and inflammation-driven tumor progression. Nat Protoc. 2007;2(8):1998-2004. doi: 10.1038/nprot.2007.279. PubMed PMID: 17703211.

314. Kim JJ, Shajib MS, Manocha MM, Khan WI. Investigating intestinal inflammation in DSS-induced model of IBD. J Vis Exp. 2012;(60). doi: 10.3791/3678. PubMed PMID: 22331082; PubMed Central PMCID: PMCPMC3369627.

315. Wullaert A, Bonnet MC, Pasparakis M. NF-kappaB in the regulation of epithelial homeostasis and inflammation. Cell research. 2011;21(1):146-58. doi: 10.1038/cr.2010.175. PubMed PMID: 21151201; PubMed Central PMCID: PMCPMC3193399.

316. Schreiber S, Nikolaus S, Hampe J. Activation of nuclear factor kappa B inflammatory bowel disease. Gut. 1998;42(4):477-84. PubMed PMID: 9616307; PubMed Central PMCID: PMCPMC1727068.

317. Neurath MF, Pettersson S, Meyer zum Buschenfelde KH, Strober W. Local administration of antisense phosphorothioate oligonucleotides to the p65 subunit of NF-kappa B abrogates established experimental colitis in mice. Nature medicine. 1996;2(9):998-1004. PubMed PMID: 8782457.

318. Karrasch T, Kim JS, Muhlbauer M, Magness ST, Jobin C. Gnotobiotic IL-10-/-;NFkappa B(EGFP) mice reveal the critical role of TLR/NF-kappa B signaling in commensal bacteria-induced colitis. J Immunol. 2007;178(10):6522-32. PubMed PMID: 17475882. 
319. Li Y, Teo WL, Low MJ, Meijer L, Sanderson I, Pettersson S, et al. Constitutive TLR4 signalling in intestinal epithelium reduces tumor load by increasing apoptosis in APC(Min/+) mice. Oncogene. 2014;33(3):369-77. doi: 10.1038/onc.2012.581. PubMed PMID: 23318418. 320. Nagel JM, Geiger BM, Karagiannis AK, Gras-Miralles B, Horst D, Najarian RM, et al. Reduced intestinal tumorigenesis in APCmin mice lacking melanin-concentrating hormone. PloS one. 2012;7(7):e41914. doi: 10.1371/journal.pone.0041914. PubMed PMID: 22848656; PubMed Central PMCID: PMCPMC3407051.

321. Grobet L, Pirottin D, Farnir F, Poncelet D, Royo LJ, Brouwers B, et al. Modulating skeletal muscle mass by postnatal, muscle-specific inactivation of the myostatin gene. Genesis. 2003;35(4):227-38. doi: 10.1002/gene.10188. PubMed PMID: 12717734. 322. Guo B, Zhang ZK, Liang C, Li J, Liu J, Lu A, et al. Molecular Communication from Skeletal Muscle to Bone: A Review for Muscle-Derived Myokines Regulating Bone Metabolism. Calcif Tissue Int. 2017;100(2):184-92. doi: 10.1007/s00223-016-0209-4. PubMed PMID: 27830278.

323. Nielsen S, Pedersen BK. Skeletal muscle as an immunogenic organ. Current opinion in pharmacology. 2008;8(3):346-51. doi: 10.1016/j.coph.2008.02.005. PubMed PMID: 18417420 .

324. Day CP, Merlino G, Van Dyke T. Preclinical mouse cancer models: a maze of opportunities and challenges. Cell. 2015;163(1):39-53. doi: 10.1016/j.cell.2015.08.068. PubMed PMID: 26406370; PubMed Central PMCID: PMCPMC4583714.

325. Bill R, Fagiani E, Zumsteg A, Antoniadis H, Johansson D, Haefliger S, et al. Nintedanib Is a Highly Effective Therapeutic for Neuroendocrine Carcinoma of the Pancreas (PNET) in the Rip1Tag2 Transgenic Mouse Model. Clinical cancer research : an official journal of the American Association for Cancer Research. 2015;21(21):4856-67. doi: 10.1158/1078-0432.CCR-14-3036. PubMed PMID: 26206868.

326. Bill R, Christofori G. The Rip1Tag2 Transgenic Mouse Model. Methods Mol Biol. 2016;1464:151-61. doi: 10.1007/978-1-4939-3999-2_14. PubMed PMID: 27858364.

327. Wei Y, Chen Y, Qiu Y, Zhao H, Liu G, Zhang Y, et al. Prevention of Muscle Wasting by CRISPR/Cas9-mediated Disruption of Myostatin In Vivo. Molecular therapy: the journal of the American Society of Gene Therapy. 2016;24(11):1889-91. doi: 10.1038/mt.2016.192. PubMed PMID: 27916995; PubMed Central PMCID: PMCPMC5154488.

328. Zhang C, McFarlane C, Lokireddy S, Bonala S, Ge X, Masuda S, et al. Myostatindeficient mice exhibit reduced insulin resistance through activating the AMP-activated protein kinase signalling pathway. Diabetologia. 2011;54(6):1491-501. doi: 10.1007/s00125011-2079-7. PubMed PMID: 21347623.

329. Dankbar B, Fennen M, Brunert D, Hayer S, Frank S, Wehmeyer C, et al. Myostatin is a direct regulator of osteoclast differentiation and its inhibition reduces inflammatory joint destruction in mice. Nature medicine. 2015;21(9):1085-90. doi: 10.1038/nm.3917. PubMed PMID: 26236992. 